Supporting Information

\title{
Copper-Catalyzed Cyclization of $N$-Propargyl Ynamides with Borane Adducts through B-H Bond Insertion
}

Guang-Yu Zhu, Tong-Yi Zhai, Xiao Li, Chong-Yang Shi,* Xin-Qi Zhu, and Long-Wu Ye*

Key Laboratory for Chemical Biology of Fujian Province and State Key Laboratory of Physical Chemistry of Solid Surfaces, College of Chemistry and Chemical Engineering, Xiamen University, Xiamen 361005, China

\section{Content}

Page Number

1. General Information.

S2

2. More Reaction Condition Study S3

3. General Procedures and Transformations S5

4. References S29

5. Crystal Data and Structure Refinement for $2 \mathrm{~s}$. S30

6. ${ }^{1} \mathrm{H},{ }^{13} \mathrm{C}$ and ${ }^{19}$ F NMR 


\section{General Information}

All reactions were carried out with a Titan HMS-14 digital magnetic stirrer with hot plate. Ethyl acetate (ACS grade), hexanes (ACS grade), anhydrous 1,2-dichloroethane (ACS grade) and toluene (ACS grade) were obtained commercially and used without further purification. Methylene chloride, tetrahydrofuran and diethyl ether were purified according to standard methods unless otherwise noted. Commercially available reagents were used without further purification. Reactions were monitored by thin layer chromatography (TLC) using silicycle pre-coated silica gel plates. Flash column chromatography was performed over silica gel (200-300 mesh). Infrared spectra were recorded on a Nicolet AVATER FTIR330 spectrometer as thin film and are reported in reciprocal centimeter $\left(\mathrm{cm}^{-1}\right)$. Mass spectra were recorded with Agilent 1290-6545XT Ultra-High performance liquid chromatography-quadrupole time-of-flight mass spectrometer using electron spray ionization. X-ray diffraction analysis was recorded on a Rigaku AFC7R X-ray single crystal diffractometer. HPLC analyses were carried out in a chromatograph equipped with a UV diode-array detector using chiral stationary columns from Daicel.

${ }^{1} \mathrm{H}$ NMR spectra were recorded on a Bruker AV-400 spectrometer and a Bruker AV-500 spectrometer in chloroform- $d_{3}$. Chemical shifts are reported in ppm with the internal TMS signal at $0.0 \mathrm{ppm}$ as a standard. The data is being reported as $(\mathrm{s}=$ singlet, $\mathrm{d}=$ doublet, $\mathrm{t}=$ triplet, $\mathrm{m}=$ multiplet or unresolved, brs = broad singlet, coupling constant(s) in $\mathrm{Hz}$, integration).

${ }^{13} \mathrm{C}$ NMR spectra were recorded on a Bruker AV-400 spectrometer and a Bruker AV-500 spectrometer in chloroform- $d_{3}$. Chemical shifts are reported in ppm with the internal chloroform signal at $77.0 \mathrm{ppm}$ as a standard. 


\section{More Reaction Condition Study}

\subsection{Table S1. Screening of Other Lewis Base-Borane Adducts ${ }^{a}$}

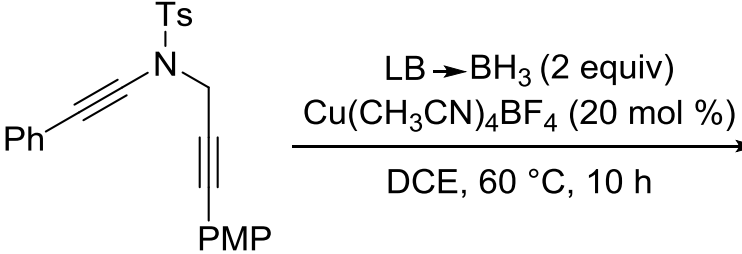

$1 b$

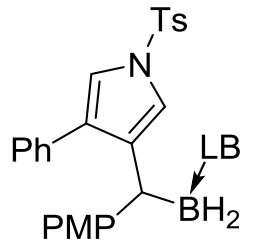

2

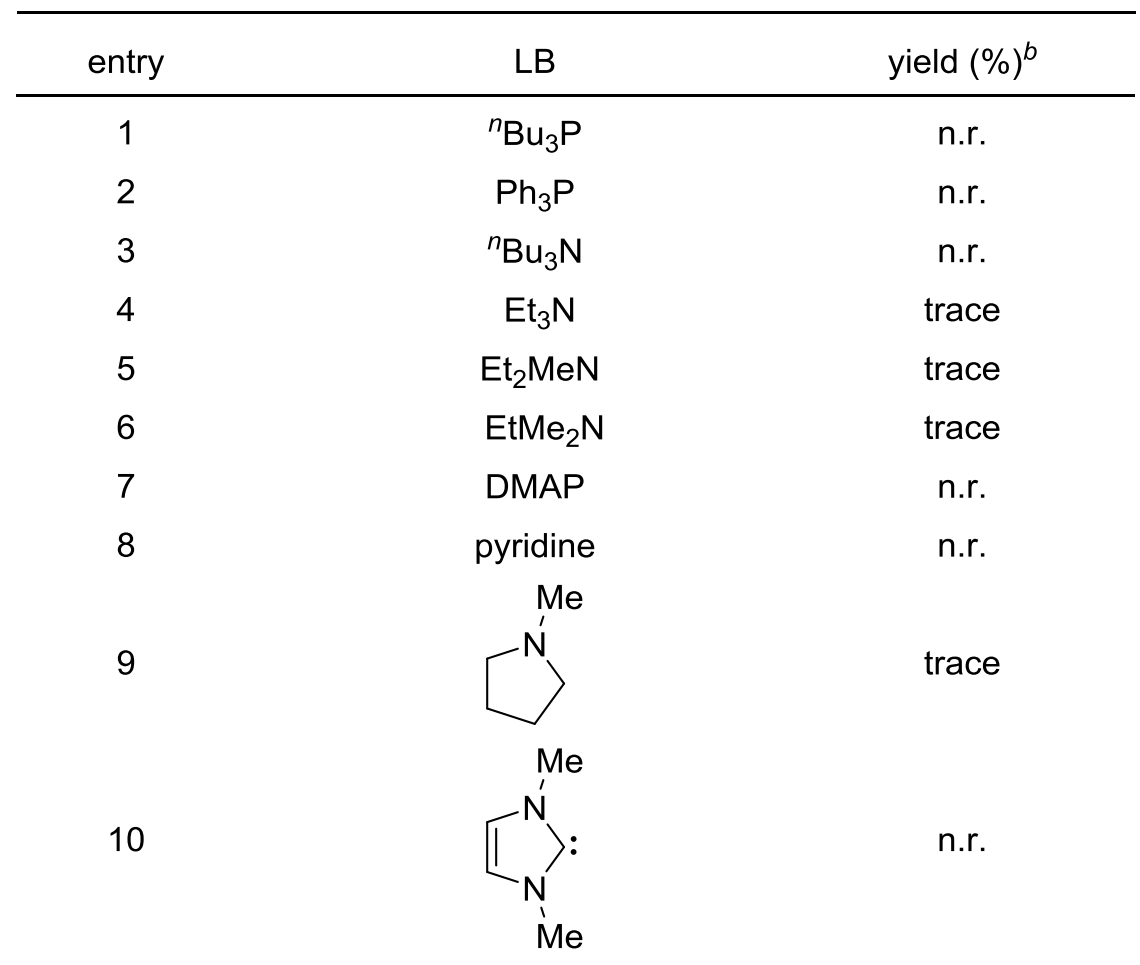

${ }^{a}$ Reaction conditions: $1 \mathrm{a}(0.05 \mathrm{mmol}), \mathrm{Cu}\left(\mathrm{CH}_{3} \mathrm{CN}\right)_{4} \mathrm{BF}_{4}(0.005 \mathrm{mmol})$, borane $(0.1 \mathrm{mmol}), \mathrm{DCE}(1 \mathrm{~mL}), 60{ }^{\circ} \mathrm{C}, 10 \mathrm{~h}$, in vials. ${ }^{b}$ Measured by ${ }^{1} \mathrm{H}$ NMR using 1,3,5-trimethoxybenzene as internal reference.

Our attempts to extend the reaction to other Lewis base-borane adducts only led to the formation of complicated mixtures or no reaction. 


\subsection{Table S2. Optimization of Reaction Conditions for the Asymmetric B-H}

\section{Bond Insertion Reaction ${ }^{a}$}

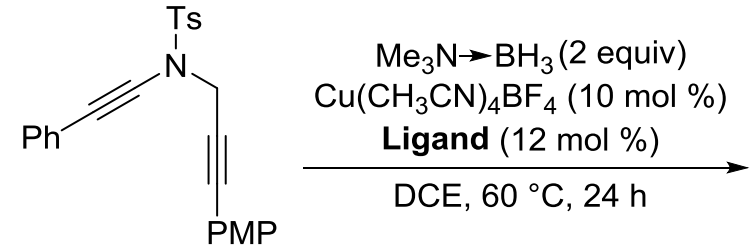

$1 \mathrm{~b}$<smiles>CC(C)(C1=N[C@@H](Br)CO1)C1=N[C@@H](Br)CO1</smiles>

L6<smiles>COc1cccc(P)c1-c1c(OC)cccc1-c1ccccc1</smiles>

ᄂ4<smiles>CC(C)(C1=N[C@@H](Cl)CO1)C1=N[C@@H](Cl)CO1</smiles>

L7<smiles>PC1=C(c2cccc(P)c2P)C2CCCCC2C=C1</smiles>

L5

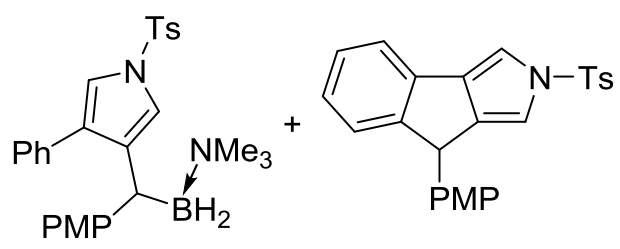

2b<smiles>BrC(C1=NC(c2ccccc2)C(c2ccccc2)O1)(C1=NC(c2ccccc2)[C@H](c2ccccc2)O1)c1ccccc1</smiles>

L8<smiles>Pc1ccccc1-c1c(-c2ccccc2)ccc2c1OCO2</smiles><smiles>Pc1ccccc1-c1c(-c2ccccc2)ccc2c1OCCO2</smiles>

L3

\begin{tabular}{cccccc}
\hline & & \multicolumn{3}{c}{ yield $(\%)^{b}$} & ee $(\%)-\mathbf{2} \mathbf{b}^{c}$ \\
\cline { 3 - 5 } entry & Ligand & $\mathbf{1 b}$ & $\mathbf{2 b}$ & $\mathbf{6}$ & - \\
\hline 1 & L1 & 5 & 11 & 64 & - \\
2 & L2 & 41 & 9 & 37 & - \\
3 & L3 & 8 & 5 & 78 & $<10$ \\
4 & L4 & 17 & 36 & 26 & $<10$ \\
5 & L5 & 27 & 27 & 36 & $<10$ \\
6 & L6 & 32 & 34 & 30 & $<10$ \\
7 & L7 & 32 & 29 & 20 & $<10$ \\
8 & L8 & 31 & 33 & 32 & . \\
\hline
\end{tabular}

${ }^{a}$ Reaction conditions: $1 \mathbf{b}(0.05 \mathrm{mmol}), \mathrm{Cu}\left(\mathrm{CH}_{3} \mathrm{CN}\right)_{4} \mathrm{BF}_{4}(0.005 \mathrm{mmol})$, ligand $(0.006 \mathrm{mmol})$, borane (0.01 mmol), DCE $(1 \mathrm{~mL}), 60{ }^{\circ} \mathrm{C}, 24 \mathrm{~h}$, in vials. ${ }^{b}$ Measured by ${ }^{1} \mathrm{H}$ NMR using $1,3,5-$ trimethoxybenzene as internal reference. ${ }^{\circ}$ Determined by HPLC analysis.

As outlined in Table S2, preliminary explorations of this asymmetric version by employing chiral BOX ligands and biphosphine ligands only led to low enantioselectivities $(<10 \%$ ee), and the significant formation of background $\mathrm{C}-\mathrm{H}$ insertion product $\mathbf{6}$ was observed in these cases. 


\section{General Procedures and Transformations}

3.1 Representative synthetic procedure for the preparation of ynamides 1 (1a-1t) 1,2
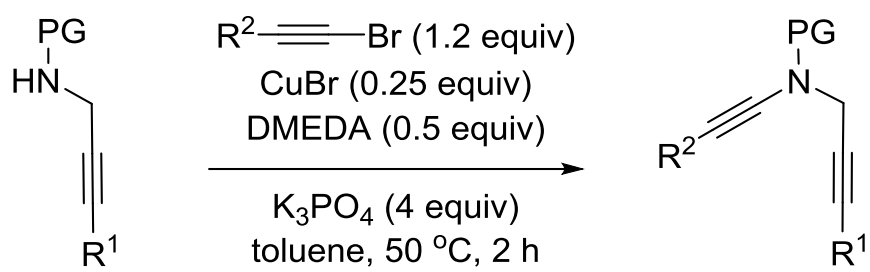

$1,60-94 \%$

To a solution of the protected propargylamine derivative ( $1 \mathrm{mmol}, 1$ equiv) in toluene (0.1 M) was added copper bromide ( $0.25 \mathrm{mmol}, 0.25$ equiv), DMEDA ( $0.5 \mathrm{mmol}, 0.5$ equiv), $\mathrm{K}_{3} \mathrm{PO}_{4}$ (4 mmol, 4 equiv), acetylene bromide derivative (1.2 mmol, 1.2 equiv). The reaction was stirred at $50{ }^{\circ} \mathrm{C}$ for about $2 \mathrm{~h}$ and the progress of the reaction was monitored by TLC. Upon completion, the solution was then filtered and concentrated under a reduced pressure. The residue was purified by silica gel column chromatography $(\mathrm{PE}: \mathrm{EA}=8: 1)$. The characterization data of ynamides 1a-1e have been reported in our previous work. ${ }^{1}$

\section{4-bromo- $N$-((4-fluorophenyl)ethynyl)- $N$-(3-(4-methoxyphenyl)prop-2-yn-1-yl)ben} zenesulfonamide (1f)

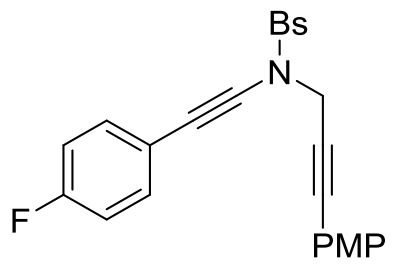

1f

Prepared according to typical procedure, after a flash column chromatography $(\mathrm{PE}: \mathrm{EA}=8: 1)$ the product $\mathbf{1 f}$ was afforded as a pale yellow oil $(77 \%, 384.5 \mathrm{mg}) .{ }^{1} \mathrm{H}$ NMR (400 MHz, $\left.\mathrm{CDCl}_{3}\right) \delta 7.87(\mathrm{~d}, J=8.8 \mathrm{~Hz}, 2 \mathrm{H}), 7.62(\mathrm{~d}, J=8.8 \mathrm{~Hz}, 2 \mathrm{H}), 7.36(\mathrm{dd}$, $J=9.2,5.6 \mathrm{~Hz}, 2 \mathrm{H}), 7.09(\mathrm{~d}, J=9.2 \mathrm{~Hz}, 2 \mathrm{H}), 6.98(\mathrm{t}, J=8.8 \mathrm{~Hz}, 2 \mathrm{H}), 6.80(\mathrm{~d}, J=8.8$ $\mathrm{Hz}, 2 \mathrm{H}), 4.56(\mathrm{~s}, 2 \mathrm{H}), 3.78(\mathrm{~s}, 3 \mathrm{H}) ;{ }^{13} \mathrm{C} \mathrm{NMR}\left(100 \mathrm{MHz}, \mathrm{CDCl}_{3}\right) \delta 162.4(\mathrm{~d}, J=$ $248.0 \mathrm{~Hz}), 159.9,136.2,133.7$ (d, $J=9.0 \mathrm{~Hz}), 133.0,132.2,129.6,129.1,118.2$ (d, $J$ 
$=3.0 \mathrm{~Hz}), 115.3(\mathrm{~d}, J=22.0 \mathrm{~Hz}), 113.9,113.6,86.9,81.2,79.3,70.2,55.2,43.2 ;{ }^{19} \mathrm{~F}$

NMR (376 MHz, $\left.\mathrm{CDCl}_{3}\right) \delta$-110.8; IR (neat): 2839, 2239, 1605, 1509, 1371, 1249, $1172,834,753,608,571 \mathrm{~cm}^{-1}$; HRESIMS Calcd for $\left[\mathrm{C}_{24} \mathrm{H}_{17} \mathrm{BrFNNaO}_{3} \mathrm{~S}\right]^{+}\left(\mathrm{M}+\mathrm{Na}^{+}\right)$ 519.9989, found 519.9985 .

\section{4-bromo- $N$-((4-chlorophenyl)ethynyl)- $N$-(3-(4-methoxyphenyl)prop-2-yn-1-yl)be} nzenesulfonamide (1g)

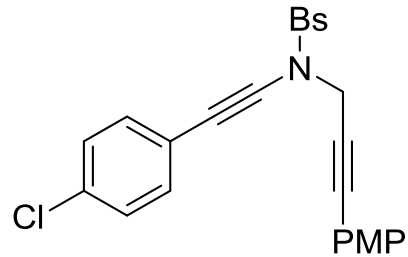

19

Prepared according to typical procedure, after a flash column chromatography $(\mathrm{PE}: \mathrm{EA}=8: 1)$ the product $1 \mathrm{~g}$ was afforded as a pale yellow oil $(65 \%, 331.2 \mathrm{mg}) .{ }^{1} \mathrm{H}$ NMR (400 MHz, $\left.\mathrm{CDCl}_{3}\right) \delta 7.87(\mathrm{~d}, J=8.4 \mathrm{~Hz}, 2 \mathrm{H}), 7.63(\mathrm{~d}, J=8.4 \mathrm{~Hz}, 2 \mathrm{H}), 7.32-$ 7.25 (m, 4H), 7.09 (d, $J=8.8 \mathrm{~Hz}, 2 \mathrm{H}), 6.80$ (d, $J=8.8 \mathrm{~Hz}, 2 \mathrm{H}), 4.56$ (s, 2H), 3.80 (s, $3 \mathrm{H}) ;{ }^{13} \mathrm{C}$ NMR $\left(100 \mathrm{MHz}, \mathrm{CDCl}_{3}\right) \delta 156.0,136.3,134.2,133.1,132.8,132.3,129.6$, 129.2, 128.6, 120.8, 114.0, 113.6, 87.0, 82.4, 79.3, 70.4, 55.3, 43.2; IR (neat): 2882, 2237, 1605, 1509, 1372, 1249, 1172, 827, 744, 607, $562 \mathrm{~cm}^{-1}$; HRESIMS Calcd for $\left[\mathrm{C}_{24} \mathrm{H}_{17} \mathrm{BrClNNaO} 3\right]^{+}\left(\mathrm{M}+\mathrm{Na}^{+}\right)$535.9693, found 535.9689.

\section{4-bromo- $N$-((4-bromophenyl)ethynyl)- $N$-(3-(4-methoxyphenyl)prop-2-yn-1-yl)be} nzenesulfonamide (1h)

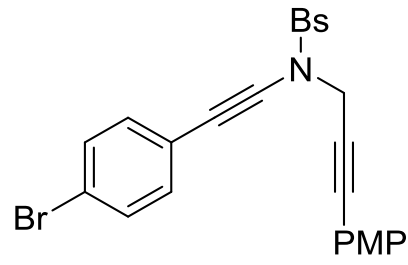

$1 \mathrm{~h}$

Prepared according to typical procedure, after a flash column chromatography $(\mathrm{PE}: \mathrm{EA}=8: 1)$ the product $\mathbf{1 h}$ was afforded as a pale yellow oil $(90 \%, 503.0 \mathrm{mg}) .{ }^{1} \mathrm{H}$ 
NMR (400 MHz, $\left.\mathrm{CDCl}_{3}\right) \delta 7.87(\mathrm{~d}, J=8.4 \mathrm{~Hz}, 2 \mathrm{H}), 7.63(\mathrm{~d}, J=8.4 \mathrm{~Hz}, 2 \mathrm{H}), 7.42$ (d, $J=8.4 \mathrm{~Hz}, 2 \mathrm{H}), 7.24(\mathrm{~d}, J=8.8 \mathrm{~Hz}, 2 \mathrm{H}), 7.09(\mathrm{~d}, J=8.8 \mathrm{~Hz}, 2 \mathrm{H}), 6.80(\mathrm{~d}, J=8.8 \mathrm{~Hz}$, 2H), $4.56(\mathrm{~s}, 2 \mathrm{H}), 3.80(\mathrm{~s}, 3 \mathrm{H}) ;{ }^{13} \mathrm{C} \mathrm{NMR}\left(100 \mathrm{MHz}, \mathrm{CDCl}_{3}\right) \delta 160.0,136.3,133.1$, 133.0, 132.3, 131.6, 129.6, 129.2, 122.3, 121.3, 114.0, 113.6, 87.0, 82.6, 79.3, 70.5, 55.3, 43.2; IR (neat): 2930, 2236, 1606, 1508, 1373, 1249, 1172, 822, 742, 603, 551 $\mathrm{cm}^{-1}$; HRESIMS Calcd for $\left[\mathrm{C}_{24} \mathrm{H}_{17} \mathrm{Br}_{2} \mathrm{NNaO}_{3} \mathrm{~S}\right]^{+}\left(\mathrm{M}+\mathrm{Na}^{+}\right)$579.9188, found 579.9192 .

4-bromo- $N$-(3-(4-methoxyphenyl)prop-2-yn-1-yl)- $N$-(p-tolylethynyl)benzenesulfo namide (1i)

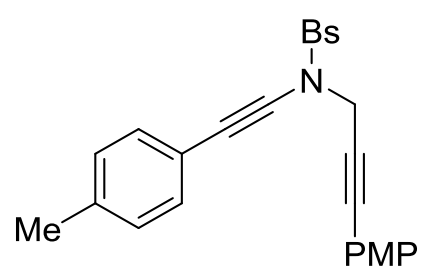

1i

Prepared according to typical procedure, after a flash column chromatography $(\mathrm{PE}: \mathrm{EA}=8: 1)$ the product $\mathbf{1 i}$ was afforded as a pale yellow oil $(88 \%, 434.4 \mathrm{mg}) .{ }^{1} \mathrm{H}$ NMR (400 MHz, $\left.\mathrm{CDCl}_{3}\right) \delta 7.87(\mathrm{~d}, J=8.4 \mathrm{~Hz}, 2 \mathrm{H}), 7.58(\mathrm{~d}, J=8.4 \mathrm{~Hz}, 2 \mathrm{H}), 7.28(\mathrm{~d}$, $J=8.0 \mathrm{~Hz}, 2 \mathrm{H}), 7.08(\mathrm{~d}, J=8.4 \mathrm{~Hz}, 4 \mathrm{H}), 6.78(\mathrm{~d}, J=8.8 \mathrm{~Hz}, 2 \mathrm{H}), 4.54(\mathrm{~s}, 2 \mathrm{H}), 3.76$ (s, 3H), $2.31(\mathrm{~s}, 3 \mathrm{H}) ;{ }^{13} \mathrm{C}$ NMR $\left(100 \mathrm{MHz}, \mathrm{CDCl}_{3}\right) \delta 159.8,138.3,136.2,133.0,132.0$, 131.6, 129.6, 129.0, 128.9, 119.0, 113.8, 113.6, 86.8, 80.8, 79.4, 71.3, 55.1, 43.2, 21.3; IR (neat): 2837, 2236, 1606, 1509, 1371, 1249, 1172, 817, 753, 608, $572 \mathrm{~cm}^{-1}$; HRESIMS Calcd for $\left[\mathrm{C}_{25} \mathrm{H}_{20} \mathrm{BrNNaO}_{3} \mathrm{~S}\right]^{+}\left(\mathrm{M}+\mathrm{Na}^{+}\right)$516.0239, found 516.0236.

\section{4-bromo- $N$-((4-methoxyphenyl)ethynyl)- $N$-(3-(4-methoxyphenyl)prop-2-yn-1-yl)} benzenesulfonamide $(\mathbf{1 j})$

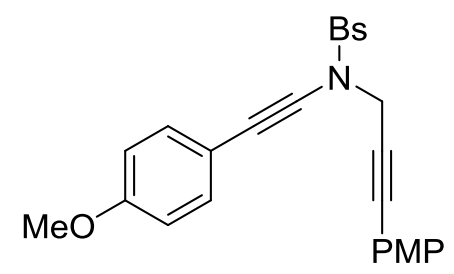

1j 
Prepared according to typical procedure, after a flash column chromatography $(\mathrm{PE}: \mathrm{EA}=8: 1)$ the product $\mathbf{1} \mathbf{j}$ was afforded as a pale yellow oil $(94 \%, 479.6 \mathrm{mg}) .{ }^{1} \mathrm{H}$ NMR (400 MHz, $\left.\mathrm{CDCl}_{3}\right) \delta 7.87(\mathrm{~d}, J=8.8 \mathrm{~Hz}, 2 \mathrm{H}), 7.61(\mathrm{~d}, J=8.4 \mathrm{~Hz}, 2 \mathrm{H}), 7.33(\mathrm{~d}$, $J=8.4 \mathrm{~Hz}, 2 \mathrm{H}), 7.09(\mathrm{~d}, J=8.8 \mathrm{~Hz}, 2 \mathrm{H}), 6.81(\mathrm{t}, J=8.8 \mathrm{~Hz}, 4 \mathrm{H}), 4.54(\mathrm{~s}, 2 \mathrm{H}), 3.79$ (s, 6H); ${ }^{13} \mathrm{C} \mathrm{NMR}\left(100 \mathrm{MHz}, \mathrm{CDCl}_{3}\right) \delta 159.9,159.7,136.3,133.6,133.0,132.1$, 129.7, 128.9, 114.1, 113.9(2), 113.9(1), 113.7, 86.8, 80.2, 79.5, 71.1, 55.2, 43.3; IR (neat): 2838, 2236, 1606, 1509, 1371, 1249, 1172, 817, 753, 608, $573 \mathrm{~cm}^{-1}$; HRESIMS Calcd for $\left[\mathrm{C}_{25} \mathrm{H}_{20} \mathrm{BrNNaO}_{4} \mathrm{~S}\right]^{+}\left(\mathrm{M}+\mathrm{Na}^{+}\right)$532.0189, found 532.0192.

\section{4-bromo- $N$-((3-fluorophenyl)ethynyl)- $N$-(3-(4-methoxyphenyl)prop-2-yn-1-yl)ben} zenesulfonamide (1k)

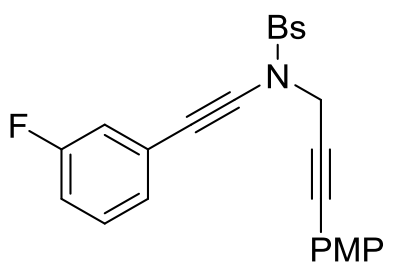

$1 \mathrm{k}$

Prepared according to typical procedure, after a flash column chromatography $(\mathrm{PE}: \mathrm{EA}=8: 1)$ the product $1 \mathbf{k}$ was afforded as a pale yellow oil $(60 \%, 296.5 \mathrm{mg}) .{ }^{1} \mathrm{H}$ NMR (400 MHz, $\left.\mathrm{CDCl}_{3}\right) \delta 7.87(\mathrm{~d}, J=8.8 \mathrm{~Hz}, 2 \mathrm{H}), 7.63(\mathrm{~d}, J=8.8 \mathrm{~Hz}, 2 \mathrm{H}), 7.28-$ $7.22(\mathrm{~m}, 1 \mathrm{H}), 7.17-7.15(\mathrm{~m}, 1 \mathrm{H}), 7.11-7.05(\mathrm{~m}, 3 \mathrm{H}), 6.99$ (tdd, $J=8.8,2.4,0.8 \mathrm{~Hz}$, $1 \mathrm{H}), 6.80(\mathrm{~d}, J=8.8 \mathrm{~Hz}, 2 \mathrm{H}), 4.57(\mathrm{~s}, 2 \mathrm{H}), 3.79(\mathrm{~s}, 3 \mathrm{H}) ;{ }^{13} \mathrm{C} \mathrm{NMR}\left(100 \mathrm{MHz}, \mathrm{CDCl}_{3}\right)$ $\delta 162.3(\mathrm{~d}, J=246.0 \mathrm{~Hz}), 160.0,136.2,133.1,132.3,129.9(\mathrm{~d}, J=8.0 \mathrm{~Hz}), 129.6$, 129.2, $127.3(\mathrm{~d}, J=3.0 \mathrm{~Hz}), 124.2(\mathrm{~d}, J=10.0 \mathrm{~Hz}), 118.2(\mathrm{~d}, J=22.0 \mathrm{~Hz}), 115.4(\mathrm{~d}, J$ $=21.0 \mathrm{~Hz}), 114.0,113.6,87.0,82.5,79.2,70.4(\mathrm{~d}, J=3.0 \mathrm{~Hz}), 55.2,43.2 ;{ }^{19} \mathrm{~F}$ NMR $\left(376 \mathrm{MHz}, \mathrm{CDCl}_{3}\right) \delta$-112.9; IR (neat): 2838, 2242, 1607, 1509, 1373, 1250, 1173, 871, 752, 608, $579 \mathrm{~cm}^{-1}$; HRESIMS Calcd for $\left[\mathrm{C}_{24} \mathrm{H}_{17} \mathrm{BrFNNaO}_{3} \mathrm{~S}\right]^{+}\left(\mathrm{M}+\mathrm{Na}^{+}\right)$ 519.9989, found 519.9992

\section{4-bromo- $N$-(3-(4-methoxyphenyl)prop-2-yn-1-yl)- $N$-(m-tolylethynyl)benzenesulfo namide (1I)}




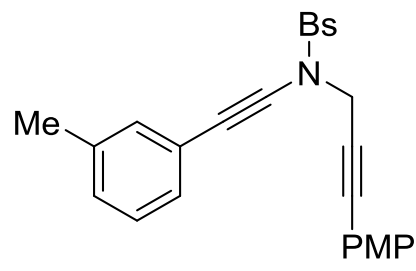

11

Prepared according to typical procedure, after a flash column chromatography $(\mathrm{PE}: \mathrm{EA}=8: 1)$ the product 11 was afforded as a pale yellow oil $(91 \%, 449.7 \mathrm{mg}) .{ }^{1} \mathrm{H}$ NMR (400 MHz, $\left.\mathrm{CDCl}_{3}\right) \delta 7.87(\mathrm{~d}, J=8.8 \mathrm{~Hz}, 2 \mathrm{H}), 7.60(\mathrm{~d}, J=8.8 \mathrm{~Hz}, 2 \mathrm{H}), 7.20-$ 7.15 (m, 3H), 7.09 (d, $J=8.8 \mathrm{~Hz}, 3 \mathrm{H}), 6.79$ (d, $J=8.8 \mathrm{~Hz}, 2 \mathrm{H}), 4.55$ (s, 2H), 3.77 (s, 3H), 2.29 (s, 3H); $\left.{ }^{13} \mathrm{C} \mathrm{NMR} \mathrm{(100} \mathrm{MHz,} \mathrm{CDCl}_{3}\right) \delta 159.8,137.9,136.2,133.0,132.1$, 129.6, 129.0, 128.6, 128.1, 122.0, 113.9, 113.7, 86.9, 81.2, 79.4, 71.4, 55.2, 43.2, 21.1; IR (neat): 2838, 2238, 1606, 1509, 1371, 1249, 1173, 832, 752, 609, $579 \mathrm{~cm}^{-1}$; HRESIMS Calcd for $\left[\mathrm{C}_{25} \mathrm{H}_{20} \mathrm{BrNNaO}_{3} \mathrm{~S}\right]^{+}\left(\mathrm{M}+\mathrm{Na}^{+}\right)$516.0239, found 516.0234.

\section{4-bromo- $N$-((2-fluorophenyl)ethynyl)- $N$-(3-(4-methoxyphenyl)prop-2-yn-1-yl)ben} zenesulfonamide (1m)

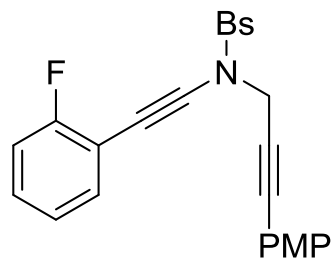

$1 \mathrm{~m}$

Prepared according to typical procedure, after a flash column chromatography $(\mathrm{PE}: \mathrm{EA}=8: 1)$ the product $\mathbf{1 m}$ was afforded as a pale yellow oil $(61 \%, 304.3 \mathrm{mg}) .{ }^{1} \mathrm{H}$ NMR $\left(400 \mathrm{MHz}, \mathrm{CDCl}_{3}\right) \delta 7.91(\mathrm{~d}, J=8.8 \mathrm{~Hz}, 2 \mathrm{H}), 7.61(\mathrm{~d}, J=8.8 \mathrm{~Hz}, 2 \mathrm{H}), 7.38(\mathrm{td}$, $J=7.6,1.6 \mathrm{~Hz}, 1 \mathrm{H}), 7.27-7.26(\mathrm{~m}, 1 \mathrm{H}), 7.10-7.05(\mathrm{~m}, 4 \mathrm{H}), 6.79(\mathrm{~d}, J=8.2 \mathrm{~Hz}$ 2H), $4.57(\mathrm{~s}, 2 \mathrm{H}), 3.78(\mathrm{~s}, 3 \mathrm{H}) ;{ }^{13} \mathrm{C} \mathrm{NMR}\left(100 \mathrm{MHz}, \mathrm{CDCl}_{3}\right) \delta 162.7(\mathrm{~d}, J=250.0$ Hz), 159.9, 136.1, 133.3, 133.1, 132.2, 129.8 (d, $J=8.0 \mathrm{~Hz}), 129.7,129.2,123.9$ (d, $J$ $=3.0 \mathrm{~Hz}), 115.4(\mathrm{~d}, J=20.0 \mathrm{~Hz}), 113.9,113.6,110.9(\mathrm{~d}, J=16.0 \mathrm{~Hz}), 87.0,86.2(\mathrm{~d}, J$ $=3.0 \mathrm{~Hz}), 79.1,65.0,55.2,43.2 ;{ }^{19} \mathrm{~F} \mathrm{NMR}\left(376 \mathrm{MHz}, \mathrm{CDCl}_{3}\right) \delta-109.7$, IR (neat): 2838, 2241, 1605, 1509, 1373, 1250, 1173, 833, 755, 608, $546 \mathrm{~cm}^{-1}$; HRESIMS Calcd for $\left[\mathrm{C}_{24} \mathrm{H}_{17} \mathrm{BrFNNaO}_{3} \mathrm{~S}\right]^{+}\left(\mathrm{M}+\mathrm{Na}^{+}\right)$519.9989, found 519.9981. 

namide (1n)

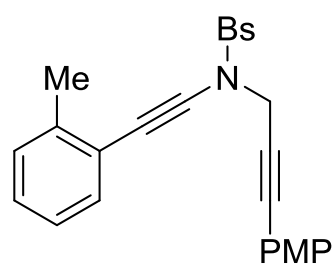

1n

Prepared according to typical procedure, after a flash column chromatography $(\mathrm{PE}: \mathrm{EA}=8: 1)$ the product 1 n was afforded as a pale yellow oil $(78 \%, 382.8 \mathrm{mg}) .{ }^{1} \mathrm{H}$ NMR (400 MHz, $\left.\mathrm{CDCl}_{3}\right) \delta 7.88(\mathrm{~d}, J=8.8 \mathrm{~Hz}, 2 \mathrm{H}), 7.59(\mathrm{~d}, J=8.8 \mathrm{~Hz}, 2 \mathrm{H}), 7.36(\mathrm{~d}$, $J=7.2 \mathrm{~Hz}, 1 \mathrm{H}), 7.20-7.10(\mathrm{~m}, 3 \mathrm{H}), 7.07(\mathrm{~d}, J=8.8 \mathrm{~Hz}, 2 \mathrm{H}), 6.79(\mathrm{~d}, J=8.8 \mathrm{~Hz}$, 2H), 4.57 (s, 2H), 3.77 (s, 3H), 2.34 (s, 3H); ${ }^{13} \mathrm{C}$ NMR (100 MHz, $\left.\mathrm{CDCl}_{3}\right) \delta 159.8$, $140.1,136.3,133.0,132.1,131.7,129.6,129.3,129.0,128.1,125.5,122.0,113.9$, 113.6, 86.8, 85.2, 79.4, 70.2, 55.2, 43.3, 20.6; IR (neat): 2838, 2233, 1606, 1509, $1371,1248,1172,832,753,607,535 \mathrm{~cm}^{-1}$; HRESIMS Calcd for $\left[\mathrm{C}_{25} \mathrm{H}_{20} \mathrm{BrNNaO}_{3} \mathrm{~S}\right]^{+}$ $\left(\mathrm{M}+\mathrm{Na}^{+}\right) 516.0239$, found 516.0242.

\section{4-bromo- $N$-(3-(4-methoxyphenyl)prop-2-yn-1-yl)- $N$-(naphthalen-1-ylethynyl)ben} zenesulfonamide (10)

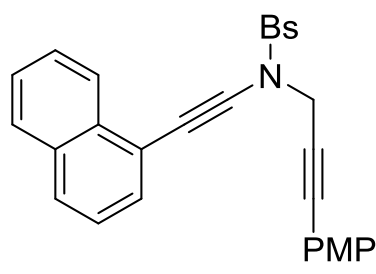

10

Prepared according to typical procedure, after a flash column chromatography $(\mathrm{PE}: \mathrm{EA}=8: 1)$ the product 10 was afforded as a pale yellow oil $(91 \%, 482.2 \mathrm{mg}) .{ }^{1} \mathrm{H}$ NMR (400 MHz, $\left.\mathrm{CDCl}_{3}\right) \delta 8.21(\mathrm{~d}, J=8.4 \mathrm{~Hz}, 1 \mathrm{H}), 7.90(\mathrm{~d}, J=8.4 \mathrm{~Hz}, 2 \mathrm{H}), 7.77(\mathrm{t}$, $J=8.0 \mathrm{~Hz}, 2 \mathrm{H}), 7.63(\mathrm{~d}, J=6.8 \mathrm{~Hz}, 1 \mathrm{H}), 7.55(\mathrm{~d}, J=8.4 \mathrm{~Hz}, 2 \mathrm{H}), 7.44(\mathrm{t}, J=7.6 \mathrm{~Hz}$, 1H), $7.34(\mathrm{dt}, J=22.4,7.6 \mathrm{~Hz}, 2 \mathrm{H}), 7.10(\mathrm{~d}, J=8.4 \mathrm{~Hz}, 2 \mathrm{H}), 6.78(\mathrm{~d}, J=8.4 \mathrm{~Hz}, 2 \mathrm{H})$, $4.64(\mathrm{~s}, 2 \mathrm{H}), 3.75$ (s, 3H); ${ }^{13} \mathrm{C}$ NMR $\left(100 \mathrm{MHz}, \mathrm{CDCl}_{3}\right) \delta 159.9,136.1,133.3,133.1$, $133.0,132.2,130.3,129.7,129.1,128.7,128.2,126.7,126.3,126.0,125.1,119.8$, 113.9, 113.6, 87.1, 86.0, 79.5, 69.6, 55.2, 43.3; IR (neat): 2838, 2235, 1606, 1509, 
$1371,1250,1173,833,750,608,539 \mathrm{~cm}^{-1}$; HRESIMS Calcd for $\left[\mathrm{C}_{28} \mathrm{H}_{20} \mathrm{BrNNaO}_{3} \mathrm{~S}\right]^{+}$ $\left(\mathrm{M}+\mathrm{Na}^{+}\right)$552.0239, found 552.0236.

\section{4-bromo- $N$-(3-(4-methoxyphenyl)prop-2-yn-1-yl)- $N$-(thiophen-2-ylethynyl)benze} nesulfonamide (1p)

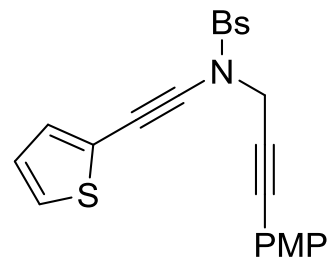

$1 \mathbf{p}$

Prepared according to typical procedure, after a flash column chromatography $(\mathrm{PE}: \mathrm{EA}=8: 1)$ the product $1 \mathbf{p}$ was afforded as a pale yellow oil $(74 \%, 361.1 \mathrm{mg}) .{ }^{1} \mathrm{H}$ NMR (400 MHz, CDCl $) \delta 7.86(\mathrm{~d}, J=8.8 \mathrm{~Hz}, 2 \mathrm{H}), 7.61(\mathrm{~d}, J=8.8 \mathrm{~Hz}, 2 \mathrm{H}), 7.27(\mathrm{dd}$, $J=5.2,1.2 \mathrm{~Hz}, 1 \mathrm{H}), 7.20(\mathrm{dd}, J=3.6,1.2 \mathrm{~Hz}, 1 \mathrm{H}), 7.10(\mathrm{~d}, J=8.8 \mathrm{~Hz}, 2 \mathrm{H}), 6.96(\mathrm{dd}$, $J=5.2,3.6 \mathrm{~Hz}, 1 \mathrm{H}), 6.80(\mathrm{~d}, J=8.8 \mathrm{~Hz}, 2 \mathrm{H}), 4.55(\mathrm{~s}, 2 \mathrm{H}), 3.79(\mathrm{~s}, 3 \mathrm{H}) ;{ }^{13} \mathrm{C} \mathrm{NMR}$ $\left(100 \mathrm{MHz}, \mathrm{CDCl}_{3}\right) \delta 159.9,136.2,133.4,133.1,132.2,129.6,129.1,128.2,127.0$, 122.2, 113.9, 113.6, 87.0, 85.2, 79.3, 64.9, 55.2, 43.4; IR (neat): 2838, 2231, 1605, 1509, 1370, 1250, 1175, 833, 750, 604, $539 \mathrm{~cm}^{-1}$; HRESIMS Calcd for $\left[\mathrm{C}_{22} \mathrm{H}_{16} \mathrm{BrNNaO}_{3} \mathrm{~S}_{2}\right]^{+}\left(\mathrm{M}+\mathrm{Na}^{+}\right)$507.9647, found 507.9650.

\section{4-bromo- $N$-(cyclopropylethynyl)- $N$-(3-(4-methoxyphenyl)prop-2-yn-1-yl)benzene} sulfonamide (1q)

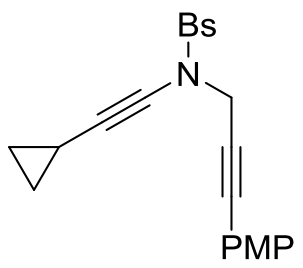

$1 \mathbf{q}$

Prepared according to typical procedure, after a flash column chromatography $(\mathrm{PE}: \mathrm{EA}=8: 1)$ the product $1 \mathrm{q}$ was afforded as a pale yellow oil $(65 \%, 288.8 \mathrm{mg}) .{ }^{1} \mathrm{H}$ NMR (400 MHz, $\left.\mathrm{CDCl}_{3}\right) \delta 7.83(\mathrm{~d}, J=8.8 \mathrm{~Hz}, 2 \mathrm{H}), 7.61(\mathrm{~d}, J=8.8 \mathrm{~Hz}, 2 \mathrm{H}), 7.07(\mathrm{~d}$, $J=8.8 \mathrm{~Hz}, 2 \mathrm{H}), 6.81(\mathrm{~d}, J=8.8 \mathrm{~Hz}, 2 \mathrm{H}), 4.42(\mathrm{~s}, 2 \mathrm{H}), 3.81(\mathrm{~s}, 3 \mathrm{H}), 1.35-1.29(\mathrm{~m}$, $1 \mathrm{H}), 0.83-0.78(\mathrm{~m}, 2 \mathrm{H}), 0.67-0.63(\mathrm{~m}, 2 \mathrm{H}) ;{ }^{13} \mathrm{C} \mathrm{NMR}\left(100 \mathrm{MHz}, \mathrm{CDCl}_{3}\right) \delta 159.8$, 
136.3, 133.0, 132.0, 129.7, 128.8, 113.9(2), 113.8(5), 86.5, 79.7, 75.7, 68.0, 55.3, 43.2, 8.9, -0.8; IR (neat): 2838, 2246, 1606, 1509, 1368, 1248, 1172, 832, 764, 608, 571 $\mathrm{cm}^{-1}$; HRESIMS Calcd for $\left[\mathrm{C}_{21} \mathrm{H}_{18} \mathrm{BrNNaO}_{3} \mathrm{~S}\right]^{+}\left(\mathrm{M}+\mathrm{Na}^{+}\right)$466.0083, found 466.0079 .

$N$-(3-(4-(benzyloxy)phenyl)prop-2-yn-1-yl)-4-bromo- $N$-(phenylethynyl)benzenes ulfonamide (1r)

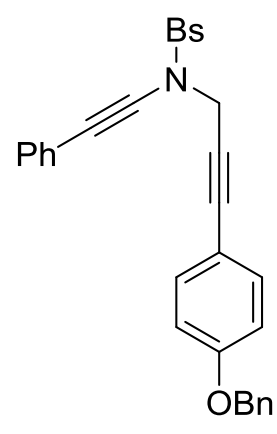

1r

Prepared according to typical procedure, after a flash column chromatography $(\mathrm{PE}: \mathrm{EA}=8: 1)$ the product 1r was afforded as a pale yellow oil $(60 \%, 331.2 \mathrm{mg}) .{ }^{1} \mathrm{H}$ NMR $\left(400 \mathrm{MHz}, \mathrm{CDCl}_{3}\right) \delta 7.87(\mathrm{~d}, J=9.2 \mathrm{~Hz}, 2 \mathrm{H}), 7.58(\mathrm{~d}, J=8.8 \mathrm{~Hz}, 2 \mathrm{H}), 7.40-$ $7.27(\mathrm{~m}, 10 \mathrm{H}), 7.08(\mathrm{~d}, J=8.8 \mathrm{~Hz}, 2 \mathrm{H}), 6.86(\mathrm{~d}, J=9.2 \mathrm{~Hz}, 2 \mathrm{H}), 5.03(\mathrm{~s}, 2 \mathrm{H}), 4.54(\mathrm{~s}$, $2 \mathrm{H}) ;{ }^{13} \mathrm{C} \mathrm{NMR}\left(100 \mathrm{MHz}, \mathrm{CDCl}_{3}\right) \delta 159.0,136.4,136.2,133.1,132.1,131.6,129.6$, $129.1,128.6,128.3,128.1,128.0,127.4,122.2,114.8,113.9,86.8,81.5,79.5,71.3$, 69.9, 43.2; IR (neat): 2928, 2236, 1605, 1508, 1372, 1244, 1172, 831, 757, 614, 535 $\mathrm{cm}^{-1}$; HRESIMS Calcd for $\left[\mathrm{C}_{30} \mathrm{H}_{22} \mathrm{BrNNaO}_{3} \mathrm{~S}\right]^{+}\left(\mathrm{M}+\mathrm{Na}^{+}\right)$578.0396, found 578.0391.

$N$-(3-(benzo $[d][1,3]$ dioxol-5-yl)prop-2-yn-1-yl)-4-bromo- $N$-(phenylethynyl)benze nesulfonamide (1s) 


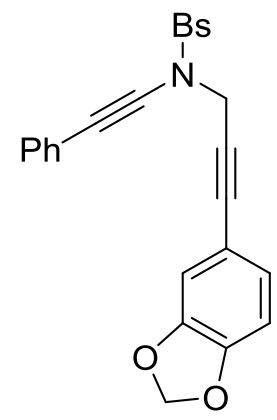

1s

Prepared according to typical procedure, after a flash column chromatography $(\mathrm{PE}: \mathrm{EA}=8: 1)$ the product $1 \mathrm{~s}$ was afforded as a pale yellow oil $(69 \%, 340.8 \mathrm{mg}){ }^{1} \mathrm{H}$ NMR (400 MHz, $\left.\mathrm{CDCl}_{3}\right) \delta 7.88(\mathrm{~d}, J=8.8 \mathrm{~Hz}, 2 \mathrm{H}), 7.62(\mathrm{~d}, J=8.4 \mathrm{~Hz}, 2 \mathrm{H}), 7.40-$ $7.38(\mathrm{~m}, 2 \mathrm{H}), 7.30-7.28(\mathrm{~m}, 3 \mathrm{H}), 6.72-6.65(\mathrm{~m}, 2 \mathrm{H}), 6.60(\mathrm{~d}, J=1.2 \mathrm{~Hz}, 1 \mathrm{H}), 5.95$ $(\mathrm{s}, 2 \mathrm{H}), 4.54(\mathrm{~s}, 2 \mathrm{H}) ;{ }^{13} \mathrm{C} \mathrm{NMR}\left(100 \mathrm{MHz}, \mathrm{CDCl}_{3}\right) \delta 148.2,147.3,136.2,132.2,131.6$, 129.6, 129.1, 128.3, 128.2, 126.4, 122.2, 114.8, 111.4, 108.4, 101.4, 86.8, 81.5, 79.1, 71.3, 43.1; IR (neat): 2901, 2234, 1573, 1489, 1371, 1249, 1174 922, 758, 618, 528 $\mathrm{cm}^{-1}$; HRESIMS Calcd for $\left[\mathrm{C}_{24} \mathrm{H}_{16} \mathrm{BrNNaO}_{4} \mathrm{~S}\right]^{+}\left(\mathrm{M}+\mathrm{Na}^{+}\right)$515.9876, found 515.9873.

\section{4-bromo- $N$-(3-(4-(dimethylamino)phenyl)prop-2-yn-1-yl)- $N$-(phenylethynyl)benz} enesulfonamide (1t)

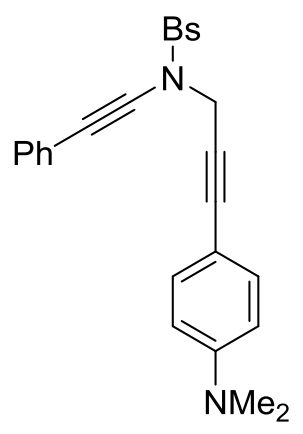

1t

Prepared according to typical procedure, after a flash column chromatography $(\mathrm{PE}: \mathrm{EA}=8: 1)$ the product $\mathbf{1 t}$ was afforded as a pale yellow oil $(75 \%, 368.4 \mathrm{mg}) .{ }^{1} \mathrm{H}$ NMR $\left(400 \mathrm{MHz}, \mathrm{CDCl}_{3}\right) \delta 7.88(\mathrm{~d}, J=8.8 \mathrm{~Hz}, 2 \mathrm{H}), 7.61(\mathrm{~d}, J=8.4 \mathrm{~Hz}, 2 \mathrm{H}), 7.40-$ $7.37(\mathrm{~m}, 2 \mathrm{H}), 7.29-7.27(\mathrm{~m}, 3 \mathrm{H}), 7.03(\mathrm{~d}, J=8.8 \mathrm{~Hz}, 2 \mathrm{H}), 6.56(\mathrm{~d}, J=8.8 \mathrm{~Hz}, 2 \mathrm{H})$, 4.57 (s, 2H), 2.95 (s, 6H); ${ }^{13} \mathrm{C}$ NMR (100 MHz, $\left.\mathrm{CDCl}_{3}\right) \delta 150.3,136.3,132.7,132.1$, 131.6, 129.7, 129.0, 128.2, 128.1, 122.4, 111.6, 108.2, 88.1, 81.7, 78.4, 71.3, 43.5, 
40.1; IR (neat): 2919, 2235, 1608, 1522, 1370, 1172, 818, 740, 601, $578 \mathrm{~cm}^{-1}$; HRESIMS Calcd for $\left[\mathrm{C}_{25} \mathrm{H}_{21} \mathrm{BrN}_{2} \mathrm{NaO}_{2} \mathrm{~S}\right]^{+}\left(\mathrm{M}+\mathrm{Na}^{+}\right)$515.0399, found 515.0394.

3.2 General procedure $B$ for the synthesis of organoboron compound 2:
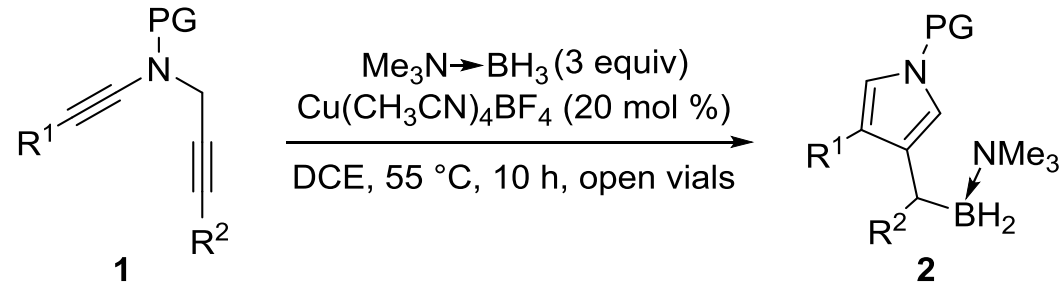

$\mathrm{Cu}\left(\mathrm{CH}_{3} \mathrm{CN}\right)_{4} \mathrm{BF}_{4}(0.02 \mathrm{mmol}, 20 \mathrm{~mol} \%)$ was added to a solution of the $N$-propargyl ynamide 1 ( $0.1 \mathrm{mmol}, 1$ equiv) and trimethylamine borane ( $0.3 \mathrm{mmol}, 3$ equiv) in DCE $(0.1 \mathrm{M})$ at room temperature. The reaction mixture was stirred at $55^{\circ} \mathrm{C}$ in open vials, and the progress of the reaction was monitored by TLC. Upon completion, the mixture was then concentrated and the residue was purified by chromatography on silicagel (PE:EA = 5:1) to afford the desired organoboron compound 2.

\section{3-(trimethylamine-boranyl(4-methoxyphenyl)methyl)-1-((4-bromophenyl)sulfony} l)-4-phenyl-1H-pyrrole (2a)

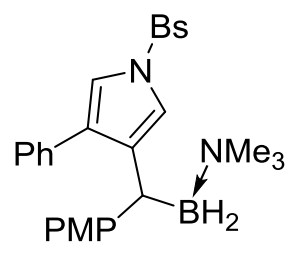

$\mathbf{2 a}$

Compound 2a was prepared in $65 \%$ yield $(36.1 \mathrm{mg}$ ) according to the general procedure (Scheme 2, entry 1). The product was isolated through silica gel column chromatography $(\mathrm{PE}: \mathrm{EA}=5: 1)$ as a white solid $\left(\mathrm{mp} 138-139{ }^{\circ} \mathrm{C}\right) .{ }^{1} \mathrm{H} \mathrm{NMR}(400 \mathrm{MHz}$, $\left.\mathrm{CDCl}_{3}\right) \delta 7.69(\mathrm{~d}, J=8.8 \mathrm{~Hz}, 2 \mathrm{H}), 7.59(\mathrm{~d}, J=8.8 \mathrm{~Hz}, 2 \mathrm{H}), 7.33-7.27$ (m, 3H), 7.24 $-7.19(\mathrm{~m}, 3 \mathrm{H}), 7.03-7.00(\mathrm{~m}, 3 \mathrm{H}), 6.72(\mathrm{~d}, J=8.8 \mathrm{~Hz}, 2 \mathrm{H}), 3.75(\mathrm{~s}, 3 \mathrm{H}), 3.19(\mathrm{t}, J=$ $4.8 \mathrm{~Hz}, 1 \mathrm{H}), 2.27$ (s, 9H); ${ }^{13} \mathrm{C} \mathrm{NMR}\left(100 \mathrm{MHz}, \mathrm{CDCl}_{3}\right) \delta 156.3,141.3,138.3,136.4$, 134.6, 132.4, 132.0, 129.1, 128.9, 128.5, 128.1, 128.0, 126.8, 119.1, 117.7, 113.4, 55.2, 52.5, 33.1; ${ }^{11} \mathrm{~B}$ NMR (128 MHz, $\left.\mathrm{CDCl}_{3}\right) \delta 2.51$; IR (neat): 2943, 2352, 1506, 
1370, 1243, 1175, 1068, 841, 745, $657 \mathrm{~cm}^{-1}$; HRESIMS Calcd for $\left[\mathrm{C}_{27} \mathrm{H}_{30} \mathrm{BBrN}_{2} \mathrm{NaO}_{3} \mathrm{~S}\right]^{+}\left(\mathrm{M}+\mathrm{Na}^{+}\right)$575.1146, found 575.1152.

\section{3-(trimethylamine-boranyl(4-methoxyphenyl)methyl)-4-phenyl-1-tosyl-1H-pyrro} le (2b)<smiles></smiles>

$2 b$

Compound $2 \mathbf{b}$ was prepared in $45 \%$ yield $(22.2 \mathrm{mg}$ ) according to the general procedure (Scheme 2, entry 2). The product was isolated through silica gel column chromatography $(\mathrm{PE}: \mathrm{EA}=5: 1)$ as a white solid $\left(\mathrm{mp} 152-153{ }^{\circ} \mathrm{C}\right) .{ }^{1} \mathrm{H}$ NMR $(400 \mathrm{MHz}$, $\left.\mathrm{CDCl}_{3}\right) \delta 7.73(\mathrm{~d}, J=8.4 \mathrm{~Hz}, 2 \mathrm{H}), 7.36-7.22(\mathrm{~m}, 8 \mathrm{H}), 7.06-7.03(\mathrm{~m}, 3 \mathrm{H}), 6.72(\mathrm{~d}$, $J=8.4 \mathrm{~Hz}, 2 \mathrm{H}), 3.75(\mathrm{~s}, 3 \mathrm{H}), 3.19(\mathrm{t}, J=4.8 \mathrm{~Hz}, 1 \mathrm{H}), 2.38(\mathrm{~s}, 3 \mathrm{H}), 2.27(\mathrm{~s}, 9 \mathrm{H}) ;{ }^{13} \mathrm{C}$ NMR $\left(100 \mathrm{MHz}, \mathrm{CDCl}_{3}\right) \delta 156.2,144.4,141.5,136.5,135.3,134.8,131.4,129.7$, $129.0,128.8,128.0,126.7,126.6,119.3,117.6,113.4,55.2,52.5,34.2,21.5 ;{ }^{11} \mathrm{~B}$ NMR (128 MHz, $\left.\mathrm{CDCl}_{3}\right) \delta 3.58$; IR (neat): 2943, 2352, 1506, 1376, 1243, 1171, 1068 , 841, 745, $657 \mathrm{~cm}^{-1}$; HRESIMS Calcd for $\left[\mathrm{C}_{28} \mathrm{H}_{33} \mathrm{BBrN}_{2} \mathrm{NaO}_{3} \mathrm{~S}^{+}\left(\mathrm{M}+\mathrm{Na}^{+}\right)\right.$511.2197, found 511.2189 .

\section{3-(trimethylamine-boranyl(4-methoxyphenyl)methyl)-1-(methylsulfonyl)-4-phen} yl-1H-pyrrole (2c)

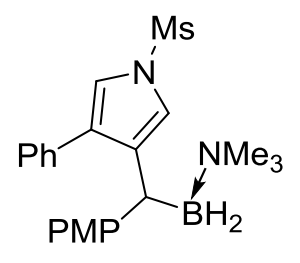

2c

Compound $2 \mathbf{c}$ was prepared in $58 \%$ yield $(23.7 \mathrm{mg})$ according to the general procedure (Scheme 2, entry 3). The product was isolated through silica gel column chromatography $(\mathrm{PE}: \mathrm{EA}=5: 1)$ as a white solid $\left(\mathrm{mp} 161-162{ }^{\circ} \mathrm{C}\right) .{ }^{1} \mathrm{H}$ NMR $(400 \mathrm{MHz}$, 
$\left.\mathrm{CDCl}_{3}\right) \delta 7.36-7.29(\mathrm{~m}, 5 \mathrm{H}), 7.18(\mathrm{~d}, J=8.8 \mathrm{~Hz}, 2 \mathrm{H}), 7.12(\mathrm{~d}, J=2.0 \mathrm{~Hz}, 1 \mathrm{H}), 7.00$ $(\mathrm{d}, J=2.8 \mathrm{~Hz}, 1 \mathrm{H}), 6.78(\mathrm{~d}, J=8.4 \mathrm{~Hz}, 2 \mathrm{H}), 3.76(\mathrm{~s}, 3 \mathrm{H}), 3.27(\mathrm{t}, J=4.8 \mathrm{~Hz}, 1 \mathrm{H})$, 3.11 (s, 3H), 2.30 (s, 9H); ${ }^{13} \mathrm{C}$ NMR (100 MHz, $\left.\mathrm{CDCl}_{3}\right) \delta 156.4,141.6,135.8,135.0$, 131.0, 129.2(2), 129.1(5), 128.1, 126.7, 118.1, 117.3, 113.6, 55.2, 52.5, 42.4, 32.8; ${ }^{11} \mathrm{~B}$ NMR (128 MHz, $\left.\mathrm{CDCl}_{3}\right) \delta$ 3.64; IR (neat): 2930, 2351, 1506, 1360, 1243, 1169 , 1070, 770, $561 \mathrm{~cm}^{-1}$; HRESIMS Calcd for $\left[\mathrm{C}_{22} \mathrm{H}_{29} \mathrm{BN}_{2} \mathrm{NaO}_{3} \mathrm{~S}\right]^{+}\left(\mathrm{M}+\mathrm{Na}^{+}\right) 435.1884$, found 435.1880 .

3-(trimethylamine-boranyl(4-methoxyphenyl)methyl)-1-((4-methoxyphenyl)sulfo nyl)-4-phenyl-1H-pyrrole (2d)

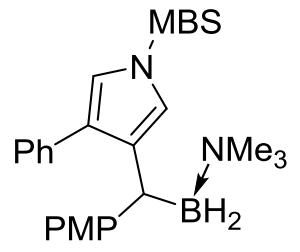

2d

Compound 2d was prepared in $41 \%$ yield $(20.4 \mathrm{mg}$ ) according to the general procedure (Scheme 2, entry 4). The product was isolated through silica gel column chromatography $(\mathrm{PE}: \mathrm{EA}=5: 1)$ as a white solid $\left(\mathrm{mp} 143-144{ }^{\circ} \mathrm{C}\right) .{ }^{1} \mathrm{H}$ NMR $(400 \mathrm{MHz}$, $\left.\mathrm{CDCl}_{3}\right) \delta 7.79(\mathrm{~d}, J=8.4 \mathrm{~Hz}, 2 \mathrm{H}), 7.31-7.22(\mathrm{~m}, 6 \mathrm{H}), 7.05-7.03(\mathrm{~m}, 3 \mathrm{H}), 6.91(\mathrm{~d}, J$ $=8.8 \mathrm{~Hz}, 2 \mathrm{H}), 6.72(\mathrm{~d}, J=8.8 \mathrm{~Hz}, 2 \mathrm{H}), 3.82(\mathrm{~s}, 3 \mathrm{H}), 3.74(\mathrm{~s}, 3 \mathrm{H}), 3.19(\mathrm{t}, J=4.8 \mathrm{~Hz}$, 1H), 2.27 (s, 9H); ${ }^{13} \mathrm{C}$ NMR (100 MHz, $\left.\mathrm{CDCl}_{3}\right) \delta 163.4,156.2,141.6,135.2,134.9$, 131.3, 131.0, 129.01, 128.95, 128.8, 128.0, 126.6, 119.2, 117.6, 114.3, 113.4, 55.6, 55.2, 52.5, 33.0; ${ }^{11} \mathrm{~B}$ NMR (128 MHz, $\left.\mathrm{CDCl}_{3}\right) \delta$ 2.66; IR (neat): 2918, 2346, 1506, $1362,1261,1164,1067,679,577 \mathrm{~cm}^{-1}$; HRESIMS Calcd for $\left[\mathrm{C}_{28} \mathrm{H}_{33} \mathrm{BN}_{2} \mathrm{NaO}_{4} \mathrm{~S}\right]^{+}(\mathrm{M}$ $+\mathrm{Na}^{+}$) 527.2146, found 527.2144.

3-(trimethylamine-boranyl(4-methoxyphenyl)methyl)-4-phenyl-1-(phenylsulfony l)-1H-pyrrole (2e)<smiles>CN(C)[C@@H](C(=N)O)c1cn(S(=O)(=O)c2ccccc2)cc1-c1ccccc1</smiles> 
Compound 2e was prepared in $65 \%$ yield $(30.9 \mathrm{mg}$ ) according to the general procedure (Scheme 2, entry 5). The product was isolated through silica gel column chromatography $(\mathrm{PE}: \mathrm{EA}=5: 1)$ as a white solid $\left(\mathrm{mp} 132-133{ }^{\circ} \mathrm{C}\right) .{ }^{1} \mathrm{H}$ NMR $(400 \mathrm{MHz}$, $\left.\mathrm{CDCl}_{3}\right) \delta 7.85(\mathrm{~d}, J=7.2 \mathrm{~Hz}, 2 \mathrm{H}), 7.57-7.53(\mathrm{~m}, 1 \mathrm{H}), 7.48-7.43(\mathrm{~m}, 2 \mathrm{H}), 7.30-$ $7.22(\mathrm{~m}, 6 \mathrm{H}), 7.07(\mathrm{~d}, J=2.4 \mathrm{~Hz}, 1 \mathrm{H}), 7.03(\mathrm{~d}, J=8.4 \mathrm{~Hz}, 2 \mathrm{H}), 6.71(\mathrm{~d}, J=8.4 \mathrm{~Hz}$, 2H), $3.74(\mathrm{~s}, 3 \mathrm{H}), 3.19(\mathrm{t}, J=4.8 \mathrm{~Hz}, 1 \mathrm{H}), 2.26(\mathrm{~s}, 9 \mathrm{H}) ;{ }^{13} \mathrm{C} \mathrm{NMR}\left(100 \mathrm{MHz}, \mathrm{CDCl}_{3}\right)$ $\delta 156.2,141.4,139.4,135.6,134.7,133.4,131.6,129.1,129.0,128.8,128.0,126.6(3)$, 126.6(1), 119.3, 117.7, 113.4, 55.2, 52.5, 33.1; ${ }^{11} \mathrm{~B}$ NMR (128 MHz, $\left.\mathrm{CDCl}_{3}\right) \delta 3.13$; IR (neat): 2941, 2351, 1610, 1506, 1367, 1243, 1174, 1067, 841, 728, $612 \mathrm{~cm}^{-1}$; HRESIMS Calcd for $\left[\mathrm{C}_{27} \mathrm{H}_{31} \mathrm{BN}_{2} \mathrm{NaO}_{3} \mathrm{~S}\right]^{+}\left(\mathrm{M}+\mathrm{Na}^{+}\right)$497.2041, found 497.2048.

\section{3-(trimethylamine-boranyl(4-methoxyphenyl)methyl)-1-((4-bromophenyl)sulfony} l)-4-(4-fluorophenyl)-1H-pyrrole (2f)

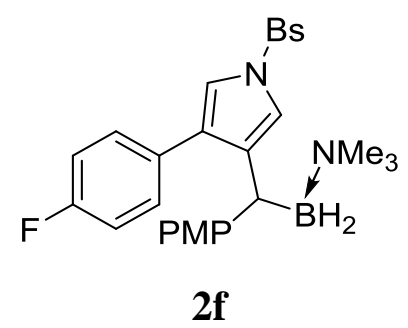

Compound 2f was prepared in $68 \%$ yield $(38.8 \mathrm{mg}$ ) according to the general procedure (Scheme 2, entry 6). The product was isolated through silica gel column chromatography $(\mathrm{PE}: \mathrm{EA}=5: 1)$ as a white solid $\left(\mathrm{mp} 189-190{ }^{\circ} \mathrm{C}\right) .{ }^{1} \mathrm{H} \mathrm{NMR}(400 \mathrm{MHz}$, $\left.\mathrm{CDCl}_{3}\right) \delta 7.70(\mathrm{~d}, J=8.4 \mathrm{~Hz}, 2 \mathrm{H}), 7.60(\mathrm{~d}, J=8.8 \mathrm{~Hz}, 2 \mathrm{H}), 7.17-7.15(\mathrm{~m}, 3 \mathrm{H}), 7.01$ $-6.97(\mathrm{~m}, 5 \mathrm{H}), 6.71(\mathrm{~d}, J=8.4 \mathrm{~Hz}, 2 \mathrm{H}), 3.75(\mathrm{~s}, 3 \mathrm{H}), 3.11(\mathrm{t}, J=4.4 \mathrm{~Hz}, 1 \mathrm{H}), 2.29$ (s, $9 \mathrm{H}) ;{ }^{13} \mathrm{C} \mathrm{NMR}\left(100 \mathrm{MHz}, \mathrm{CDCl}_{3}\right) \delta 162.0(\mathrm{~d}, J=244.0 \mathrm{~Hz}), 156.4,141.1,138.3$, $136.2,132.5,131.0,130.6(\mathrm{~d}, J=8.0 \mathrm{~Hz}), 128.9,128.6,128.2,118.4(\mathrm{~d}, J=139.0$ $\mathrm{Hz}), 114.9(\mathrm{~d}, J=21.0 \mathrm{~Hz}), 113.5,55.2,52.5,33.7 ;{ }^{11} \mathrm{~B}$ NMR $\left(128 \mathrm{MHz}, \mathrm{CDCl}_{3}\right) \delta$ 3.24; ${ }^{19} \mathrm{~F} \mathrm{NMR}\left(376 \mathrm{MHz}, \mathrm{CDCl}_{3}\right) \delta$-115.9, IR (neat): 2920, 2351, 1573, 1506, 1370, $1242,1175,1067,840,745,643 \mathrm{~cm}^{-1}$; HRESIMS Calcd for $\left[\mathrm{C}_{27} \mathrm{H}_{29} \mathrm{BBrFN}_{2} \mathrm{NaO}_{3} \mathrm{~S}\right]^{+}$ $\left(\mathrm{M}+\mathrm{Na}^{+}\right)$593.1052, found 593.1053. 

enyl)-1H-pyrrole (2g)<smiles>[B]C(c1cn([13CH3])cc1-c1ccc(Cl)cc1)N(C)C</smiles>

$2 \mathrm{~g}$

Compound $2 \mathrm{~g}$ was prepared in $73 \%$ yield $(42.8 \mathrm{mg}$ ) according to the general procedure (Scheme 2, entry 7). The product was isolated through silica gel column chromatography $(\mathrm{PE}: \mathrm{EA}=5: 1)$ as a white solid $\left(\mathrm{mp} 144-145^{\circ} \mathrm{C}\right) .{ }^{1} \mathrm{H}$ NMR $(400 \mathrm{MHz}$, $\left.\mathrm{CDCl}_{3}\right) \delta 7.70(\mathrm{~d}, J=8.8 \mathrm{~Hz}, 2 \mathrm{H}), 7.60(\mathrm{~d}, J=8.8 \mathrm{~Hz}, 2 \mathrm{H}), 7.27(\mathrm{~d}, J=8.8 \mathrm{~Hz}, 2 \mathrm{H})$, $7.20-7.14(\mathrm{~m}, 3 \mathrm{H}), 7.01-6.97(\mathrm{~m}, 3 \mathrm{H}), 6.72(\mathrm{~d}, J=8.4 \mathrm{~Hz}, 2 \mathrm{H}), 3.75(\mathrm{~s}, 3 \mathrm{H}), 3.11$ $(\mathrm{t}, J=4.0 \mathrm{~Hz}, 1 \mathrm{H}), 2.29$ (s, 9H); ${ }^{13} \mathrm{C} \mathrm{NMR}\left(100 \mathrm{MHz}, \mathrm{CDCl}_{3}\right) \delta 156.4,141.0,138.2$, $136.0,133.1,132.7,132.5,130.8,130.6,130.3$, 128.9, 128.6, 128.2, 119.2, 117.7, 113.5, 55.2, 52.5, 33.2; ${ }^{11} \mathrm{~B}$ NMR (128 MHz, $\left.\mathrm{CDCl}_{3}\right) \delta$ 4.39; IR (neat): 2943, 2384, 1574, 1506, 1372, 1243, 1175, 1091, 838, 745, $640 \mathrm{~cm}^{-1}$; HRESIMS Calcd for $\left[\mathrm{C}_{27} \mathrm{H}_{29} \mathrm{BBrClN}_{2} \mathrm{NaO}_{3} \mathrm{~S}\right]^{+}\left(\mathrm{M}+\mathrm{Na}^{+}\right)$609.0756, found 609.0761.

\section{3-(trimethylamine-boranyl(4-methoxyphenyl)methyl)-4-(4-bromophenyl)-1-((4-b} romophenyl)sulfonyl)-1H-pyrrole (2h)<smiles>[B]C(c1cn([13CH3])cc1-c1ccc(Br)cc1)N(C)C</smiles>

Compound $\mathbf{2 h}$ was prepared in $56 \%$ yield $(35.2 \mathrm{mg}$ ) according to the general procedure (Scheme 2, entry 8). The product was isolated through silica gel column chromatography $(\mathrm{PE}: \mathrm{EA}=5: 1)$ as a white solid $\left(\mathrm{mp} 130-131{ }^{\circ} \mathrm{C}\right) .{ }^{1} \mathrm{H}$ NMR $(400 \mathrm{MHz}$, $\left.\mathrm{CDCl}_{3}\right) \delta 7.70(\mathrm{~d}, J=8.8 \mathrm{~Hz}, 2 \mathrm{H}), 7.60(\mathrm{~d}, J=8.4 \mathrm{~Hz}, 2 \mathrm{H}), 7.42(\mathrm{~d}, J=8.4 \mathrm{~Hz}, 2 \mathrm{H})$, $7.19(\mathrm{~d}, J=2.4 \mathrm{~Hz}, 1 \mathrm{H}), 7.09(\mathrm{~d}, J=8.4 \mathrm{~Hz}, 2 \mathrm{H}), 7.01(\mathrm{~d}, J=2.4 \mathrm{~Hz}, 1 \mathrm{H}), 6.98$ (d, $J$ 
$=8.4 \mathrm{~Hz}, 2 \mathrm{H}), 6.72(\mathrm{~d}, J=8.4 \mathrm{~Hz}, 2 \mathrm{H}), 3.75(\mathrm{~s}, 3 \mathrm{H}), 3.11(\mathrm{t}, J=4.4 \mathrm{~Hz}, 1 \mathrm{H}), 2.29(\mathrm{~s}$, $9 \mathrm{H}) ;{ }^{13} \mathrm{C} \mathrm{NMR}\left(100 \mathrm{MHz}, \mathrm{CDCl}_{3}\right) \delta 156.4,141.0,138.2,135.9,133.6,132.5,131.2$, 130.7(4), 130.6(8), 128.9, 128.7, 128.2, 120.9, 119.2, 117.7, 113.5, 55.2, 52.5, 33.1;

${ }^{11} \mathrm{~B}$ NMR (128 MHz, $\left.\mathrm{CDCl}_{3}\right) \delta$ 4.20; IR (neat): 2942, 23552, 1506, 1372, 1246, 1174, 1068, 834, 746, $639 \mathrm{~cm}^{-1}$; HRESIMS Calcd for $\left[\mathrm{C}_{27} \mathrm{H}_{29} \mathrm{BBr}_{2} \mathrm{~N}_{2} \mathrm{NaO}_{3} \mathrm{~S}\right]^{+}\left(\mathrm{M}+\mathrm{Na}^{+}\right)$ 653.0251, found 653.0256.

3-(trimethylamine-boranyl(4-methoxyphenyl)methyl)-1-((4-bromophenyl)sulfony l)-4-(p-tolyl)-1H-pyrrole (2i)

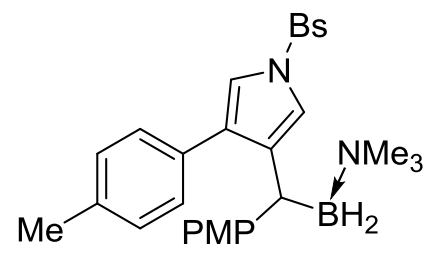

$2 \mathbf{i}$

Compound $2 \mathbf{i}$ was prepared in $71 \%$ yield (41.9 $\mathrm{mg}$ ) according to the general procedure (Scheme 2, entry 9). The product was isolated through silica gel column chromatography (PE:EA = 5:1) as a white solid $\left(\mathrm{mp} 150-151^{\circ} \mathrm{C}\right) .{ }^{1} \mathrm{H}$ NMR $(400 \mathrm{MHz}$, $\left.\mathrm{CDCl}_{3}\right) \delta 7.69(\mathrm{~d}, J=8.8 \mathrm{~Hz}, 2 \mathrm{H}), 7.59(\mathrm{~d}, J=8.8 \mathrm{~Hz}, 2 \mathrm{H}), 7.20(\mathrm{~d}, J=2.4 \mathrm{~Hz}, 1 \mathrm{H})$, $7.12(\mathrm{~s}, 4 \mathrm{H}), 7.02-7.00(\mathrm{~m}, 3 \mathrm{H}), 6.72(\mathrm{~d}, J=8.8 \mathrm{~Hz}, 2 \mathrm{H}), 3.76(\mathrm{~s}, 3 \mathrm{H}), 3.18(\mathrm{t}, J=$ $4.4 \mathrm{~Hz}, 1 \mathrm{H}), 2.35$ (s, 3H), 2.28 (s, 9H); ${ }^{13} \mathrm{C} \mathrm{NMR}\left(100 \mathrm{MHz}, \mathrm{CDCl}_{3}\right) \delta$ 156.2, 141.4, $138.3,136.4,132.4,132.0,131.5,128.9,128.8(4), 128.8(0), 128.5,128.1,119.1$, 117.6, 113.4, 55.2, 52.5, 34.1, 21.2; ${ }^{11} \mathrm{~B}$ NMR (128 MHz, $\left.\mathrm{CDCl}_{3}\right) \delta 3.52$; IR (neat): 2920, 2384, 1506, 1369, 1245, 1174, 1068, 823, 745, $643 \mathrm{~cm}^{-1}$; HRESIMS Calcd for $\left[\mathrm{C}_{28} \mathrm{H}_{32} \mathrm{BBrN}_{2} \mathrm{NaO}_{3} \mathrm{~S}\right]^{+}\left(\mathrm{M}+\mathrm{Na}^{+}\right)$589.1302, found 589.1307.

3-(trimethylamine-boranyl(4-methoxyphenyl)methyl)-1-((4-bromophenyl)sulfony l)-4-(4-methoxyphenyl)-1H-pyrrole (2j)<smiles>COc1ccc(-c2cn([135I])cc2C([15NH2])[18OH])cc1</smiles> 
Compound $\mathbf{2} \mathbf{j}$ was prepared in $72 \%$ yield $(41.7 \mathrm{mg}$ ) according to the general procedure (Scheme 2, entry 10). The product was isolated through silica gel column chromatography (PE:EA $=5: 1)$ as a white solid $\left(\mathrm{mp} 130-131{ }^{\circ} \mathrm{C}\right) .{ }^{1} \mathrm{H}$ NMR $(400 \mathrm{MHz}$, $\left.\mathrm{CDCl}_{3}\right) \delta 7.69(\mathrm{~d}, J=8.4 \mathrm{~Hz}, 2 \mathrm{H}), 7.58(\mathrm{~d}, J=8.8 \mathrm{~Hz}, 2 \mathrm{H}), 7.19(\mathrm{~d}, J=2.4 \mathrm{~Hz}, 1 \mathrm{H})$, $7.14(\mathrm{~d}, J=8.8 \mathrm{~Hz}, 2 \mathrm{H}), 7.01(\mathrm{~d}, J=9.2 \mathrm{~Hz}, 2 \mathrm{H}), 6.98(\mathrm{~d}, J=2.4 \mathrm{~Hz}, 1 \mathrm{H}), 6.84(\mathrm{~d}, J$ $=8.4 \mathrm{~Hz}, 2 \mathrm{H}), 6.72(\mathrm{~d}, J=8.4 \mathrm{~Hz}, 2 \mathrm{H}), 3.80(\mathrm{~s}, 3 \mathrm{H}), 3.75(\mathrm{~s}, 3 \mathrm{H}), 3.16(\mathrm{t}, J=4.4 \mathrm{~Hz}$,

1H), 2.28 (s, 9H); ${ }^{13} \mathrm{C}$ NMR (100 MHz, $\left.\mathrm{CDCl}_{3}\right) \delta 158.6,156.2,141.4,138.3,136.5$, 132.4, 131.7, 130.1, 128.8, 128.4, 128.1, 126.9, 119.1, 117.3, 113.5, 113.4, 55.2(0), 55.1(9), 52.5, 33.1; ${ }^{11} \mathrm{~B}$ NMR (128 MHz, $\left.\mathrm{CDCl}_{3}\right) \delta$ 2.92; IR (neat): 2919, 2352, 1506, 1369, 1246, 1174, 1067, 837, 745, $590 \mathrm{~cm}^{-1}$; HRESIMS Calcd for $\left[\mathrm{C}_{28} \mathrm{H}_{32} \mathrm{BBrN}_{2} \mathrm{NaO}_{4} \mathrm{~S}\right]^{+}\left(\mathrm{M}+\mathrm{Na}^{+}\right)$605.1251, found 605.1256.

\section{3-(trimethylamine-boranyl(4-methoxyphenyl)methyl)-1-((4-bromophenyl)sulfony} l)-4-(3-fluorophenyl)-1H-pyrrole (2k)

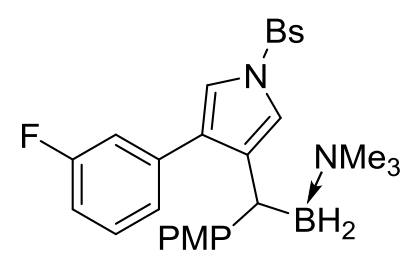

$2 \mathbf{k}$

Compound $2 \mathbf{k}$ was prepared in $44 \%$ yield $(24.8 \mathrm{mg}$ ) according to the general procedure (Scheme 2, entry 11). The product was isolated through silica gel column chromatography $(\mathrm{PE}: \mathrm{EA}=5: 1)$ as a white solid $\left(\mathrm{mp} 153-154{ }^{\circ} \mathrm{C}\right) .{ }^{1} \mathrm{H}$ NMR $(400 \mathrm{MHz}$, $\left.\mathrm{CDCl}_{3}\right) \delta 7.69(\mathrm{~d}, J=8.8 \mathrm{~Hz}, 2 \mathrm{H}), 7.60(\mathrm{~d}, J=8.8 \mathrm{~Hz}, 2 \mathrm{H}), 7.25-7.23(\mathrm{~m}, 1 \mathrm{H}), 7.18$ (d, $J=2.0 \mathrm{~Hz}, 1 \mathrm{H}), 7.03-6.95(\mathrm{~m}, 6 \mathrm{H}), 6.72(\mathrm{~d}, J=8.4 \mathrm{~Hz}, 2 \mathrm{H}), 3.75(\mathrm{~s}, 3 \mathrm{H}), 3.17$ (t, $J=4.4 \mathrm{~Hz}, 1 \mathrm{H}), 2.30(\mathrm{~s}, 9 \mathrm{H}) ;{ }^{13} \mathrm{C} \mathrm{NMR}\left(100 \mathrm{MHz}, \mathrm{CDCl}_{3}\right) \delta 162.6(\mathrm{~d}, J=244.0 \mathrm{~Hz})$, 156.4, 141.0, 138.3, 136.9 (d, $J=9.0 \mathrm{~Hz}), 136.1,132.5,130.7,129.5$ (d, $J=9.0 \mathrm{~Hz})$, 129.0, 128.6, 128.2, $124.7(\mathrm{~d}, J=2.0 \mathrm{~Hz}), 119.2,117.9,115.8(\mathrm{~d}, J=21.0 \mathrm{~Hz}), 113.6$, $113.5(\mathrm{~d}, J=21.0 \mathrm{~Hz}), 55.2,52.5,33.2 ;{ }^{11} \mathrm{~B}$ NMR $\left(128 \mathrm{MHz}, \mathrm{CDCl}_{3}\right) \delta 3.67 ;{ }^{19} \mathrm{~F}$ NMR (376 MHz, $\left.\mathrm{CDCl}_{3}\right) \delta-113.8$, IR (neat): 2942, 2335, 1574, 1506, 1372, 1243, 
1176, 1068, 745, $637 \mathrm{~cm}^{-1}$; HRESIMS Calcd for $\left[\mathrm{C}_{27} \mathrm{H}_{29} \mathrm{BBrFN}_{2} \mathrm{NaO}_{3} \mathrm{~S}\right]^{+}\left(\mathrm{M}+\mathrm{Na}^{+}\right)$ 593.1052, found 593.1059.

\section{3-(trimethylamine-boranyl(4-methoxyphenyl)methyl)-1-((4-bromophenyl)sulfony} l)-4-(m-tolyl)-1H-pyrrole (2l)<smiles>[BH3-]C([15NH2])c1cn([13CH3])cc1-c1cccc(C)c1</smiles>

21

Compound 21 was prepared in $44 \%$ yield (24.9 $\mathrm{mg}$ ) according to the general procedure (Scheme 2, entry 12). The product was isolated through silica gel column chromatography $(\mathrm{PE}: \mathrm{EA}=5: 1)$ as a white solid $\left(\mathrm{mp} 145-146{ }^{\circ} \mathrm{C}\right) .{ }^{1} \mathrm{H}$ NMR $(400 \mathrm{MHz}$, $\left.\mathrm{CDCl}_{3}\right) \delta 7.69(\mathrm{~d}, J=8.4 \mathrm{~Hz}, 2 \mathrm{H}), 7.59(\mathrm{~d}, J=8.8 \mathrm{~Hz}, 2 \mathrm{H}), 7.21-7.17(\mathrm{~m}, 2 \mathrm{H}), 7.08$ - $7.01(\mathrm{~m}, 6 \mathrm{H}), 6.73(\mathrm{~d}, J=8.8 \mathrm{~Hz}, 2 \mathrm{H}), 3.76(\mathrm{~s}, 3 \mathrm{H}), 3.18(\mathrm{t}, J=4.4 \mathrm{~Hz}, 1 \mathrm{H}), 2.31$ (s, 3H), 2.29 (s, 9H); ${ }^{13} \mathrm{C}$ NMR (100 MHz, $\left.\mathrm{CDCl}_{3}\right) \delta 156.3,141.5,138.3,137.5,136.3$, $134.4,132.4,132.2,129.8,128.9,128.5,128.1,127.9,127.5,126.0,119.2,117.6$, 113.4, 55.2, 52.5, 33.1, 21.3; ${ }^{11} \mathrm{~B}$ NMR (128 MHz, $\left.\mathrm{CDCl}_{3}\right) \delta 3.32$; IR (neat): 2918, 2352, 1506, 1370, 1242, 1175, 1068, 745, $626 \mathrm{~cm}^{-1}$; HRESIMS Calcd for $\left[\mathrm{C}_{28} \mathrm{H}_{32} \mathrm{BBrN}_{2} \mathrm{NaO}_{3} \mathrm{~S}\right]^{+}\left(\mathrm{M}+\mathrm{Na}^{+}\right)$589.1302, found 589.1305.

\section{3-(trimethylamine-boranyl(4-methoxyphenyl)methyl)-1-((4-bromophenyl)sulfony} l)-4-(2-fluorophenyl)-1H-pyrrole (2m)<smiles>CN(C)c1cn([13CH3])cc1-c1ccccc1F</smiles>

Compound $\mathbf{2 m}$ was prepared in $65 \%$ yield $(37.0 \mathrm{mg})$ according to the general procedure (Scheme 2, entry 13). The product was isolated through silica gel column chromatography $(\mathrm{PE}: \mathrm{EA}=5: 1)$ as a white solid $\left(\mathrm{mp} 158-159{ }^{\circ} \mathrm{C}\right) .{ }^{1} \mathrm{H} \mathrm{NMR}(400 \mathrm{MHz}$, 
$\left.\mathrm{CDCl}_{3}\right) \delta 7.68(\mathrm{~d}, J=8.8 \mathrm{~Hz}, 2 \mathrm{H}), 7.59(\mathrm{~d}, J=8.8 \mathrm{~Hz}, 2 \mathrm{H}), 7.25-7.22(\mathrm{~m}, 1 \mathrm{H}), 7.16$ $(\mathrm{d}, J=2.0 \mathrm{~Hz}, 1 \mathrm{H}), 7.09-7.04(\mathrm{~m}, 4 \mathrm{H}), 6.90(\mathrm{~d}, J=8.8 \mathrm{~Hz}, 2 \mathrm{H}), 6.65(\mathrm{~d}, J=8.4 \mathrm{~Hz}$, 2H), $3.73(\mathrm{~s}, 3 \mathrm{H}), 3.03(\mathrm{t}, J=4.0 \mathrm{~Hz}, 1 \mathrm{H}), 2.29(\mathrm{~s}, 9 \mathrm{H}) ;{ }^{13} \mathrm{C} \mathrm{NMR}\left(100 \mathrm{MHz}, \mathrm{CDCl}_{3}\right)$ $\delta 160.0(\mathrm{~d}, J=244.0 \mathrm{~Hz}), 156.2,141.2,138.4,137.4,132.4(1), 132.3(7)(\mathrm{d}, J=4.0$ Hz), 128.9, 128.7 (d, $J=8.0 \mathrm{~Hz}), 128.5,128.1,125.1,123.6$ (d, $J=4.0 \mathrm{~Hz}), 122.3$ (d, $J=15.0 \mathrm{~Hz}), 119.3,118.5,115.3(\mathrm{~d}, J=22.0 \mathrm{~Hz}), 113.3,55.2,52.4,33.3 ;{ }^{11} \mathrm{~B} \mathrm{NMR}$ $\left(128 \mathrm{MHz}, \mathrm{CDCl}_{3}\right) \delta 4.51 ;{ }^{19} \mathrm{~F}$ NMR $\left(376 \mathrm{MHz}, \mathrm{CDCl}_{3}\right) \delta$-114.6, IR (neat): 2943, 2355, 1574, 1371, 1243, 1175, 1068, 745, $629 \mathrm{~cm}^{-1}$; HRESIMS Calcd for $\left[\mathrm{C}_{27} \mathrm{H}_{29} \mathrm{BBrFN}_{2} \mathrm{NaO}_{3} \mathrm{~S}\right]^{+}\left(\mathrm{M}+\mathrm{Na}^{+}\right)$593.1052, found 593.1048.

\section{3-(trimethylamine-boranyl(4-methoxyphenyl)methyl)-1-((4-bromophenyl)sulfony} l)-4-(o-tolyl)-1H-pyrrole (2n)

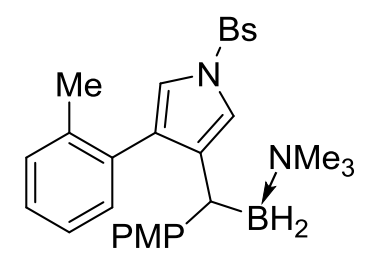

2n

Compound $2 \mathrm{n}$ was prepared in $68 \%$ yield $(38.6 \mathrm{mg}$ ) according to the general procedure (Scheme 2, entry 14). The product was isolated through silica gel column chromatography $(\mathrm{PE}: \mathrm{EA}=5: 1)$ as a white solid $\left(\mathrm{mp} 143-144{ }^{\circ} \mathrm{C}\right) .{ }^{1} \mathrm{H}$ NMR $(400 \mathrm{MHz}$, $\left.\mathrm{CDCl}_{3}\right) \delta 7.64(\mathrm{~d}, J=8.8 \mathrm{~Hz}, 2 \mathrm{H}), 7.57(\mathrm{~d}, J=8.8 \mathrm{~Hz}, 2 \mathrm{H}), 7.18-7.08(\mathrm{~m}, 4 \mathrm{H}), 6.97$ $(\mathrm{d}, J=7.2 \mathrm{~Hz}, 1 \mathrm{H}), 6.86(\mathrm{~d}, J=2.4 \mathrm{~Hz}, 1 \mathrm{H}), 6.83(\mathrm{~d}, J=8.4 \mathrm{~Hz}, 2 \mathrm{H}), 6.63(\mathrm{~d}, J=8.8$ $\mathrm{Hz}, 2 \mathrm{H}), 3.73(\mathrm{~s}, 3 \mathrm{H}), 2.78(\mathrm{t}, J=4.0 \mathrm{~Hz}, 1 \mathrm{H}), 2.23(\mathrm{~s}, 9 \mathrm{H}), 1.84(\mathrm{~s}, 3 \mathrm{H}) ;{ }^{13} \mathrm{C} \mathrm{NMR}$ $\left(100 \mathrm{MHz} \mathrm{CDCl}_{3}\right) \delta 156.3,141.2,138.5,138.1,137.4,134.0,132.2,131.3,130.9$, 129.5, 129.2, 128.2, 128.0, 127.2, 124.9, 118.2, 118.0, 113.2, 55.2, 52.4, 33.4, 20.0; ${ }^{11} \mathrm{~B}$ NMR (128 MHz, $\left.\mathrm{CDCl}_{3}\right) \delta$ 3.29; IR (neat): 2943, 2350, 1574, 1506, 1368, 1241, 1174, 1068, 744, $587 \mathrm{~cm}^{-1}$; HRESIMS Calcd for $\left[\mathrm{C}_{28} \mathrm{H}_{32} \mathrm{BBrN}_{2} \mathrm{NaO}_{3} \mathrm{~S}\right]^{+}\left(\mathrm{M}+\mathrm{Na}^{+}\right)$ 589.1032 , found 589.1036 .

3-(trimethylamine-boranyl(4-methoxyphenyl)methyl)-1-((4-bromophenyl)sulfony 1)-4-(naphthalen-1-yl)-1H-pyrrole (2o) 


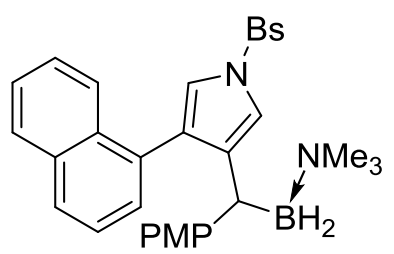

20

Compound 20 was prepared in $73 \%$ yield $(44.1 \mathrm{mg})$ according to the general procedure (Scheme 2, entry 15). The product was isolated through silica gel column chromatography (PE:EA $=5: 1)$ as a white solid $\left(\mathrm{mp} 184-185^{\circ} \mathrm{C}\right) .{ }^{1} \mathrm{H}$ NMR $(400 \mathrm{MHz}$, $\left.\mathrm{CDCl}_{3}\right) \delta 7.80(\mathrm{dd}, J=16.0,8.0 \mathrm{~Hz}, 2 \mathrm{H}), 7.70(\mathrm{~d}, J=8.8 \mathrm{~Hz}, 2 \mathrm{H}), 7.60(\mathrm{~d}, J=8.8 \mathrm{~Hz}$, 2H), $7.44-7.37$ (m, 3H), 7.29 (s, 1H), 7.15 (s, 2H), 7.02 (d, J=2.4 Hz, 1H), 6.77 (d, $J=6.4 \mathrm{~Hz}, 2 \mathrm{H}), 6.58(\mathrm{~d}, J=8.8 \mathrm{~Hz}, 2 \mathrm{H}), 3.71(\mathrm{~s}, 3 \mathrm{H}), 2.75(\mathrm{t}, J=4.0 \mathrm{~Hz}, 1 \mathrm{H}), 2.09$ (s, 9H); ${ }^{13} \mathrm{C}$ NMR (100 MHz, $\left.\mathrm{CDCl}_{3}\right) \delta 156.3,153.3,149.5,138.8(4), 138.7(9), 138.4$, $133.3,132.7,132.3,130.2,129.2,128.4,128.2,128.1,127.8,127.4,126.5,125.5$, 125.0, 119.2, 118.0, 113.2, 55.2, 52.2, 33.6; ${ }^{11} \mathrm{~B}$ NMR (128 MHz, $\left.\mathrm{CDCl}_{3}\right) \delta 3.55 ; \mathrm{IR}$ (neat): 2942, 2352, 1506, 1370, 1242, 1175, 1067, 745, $627 \mathrm{~cm}^{-1}$; HRESIMS Calcd for $\left[\mathrm{C}_{31} \mathrm{H}_{32} \mathrm{BBrN}_{2} \mathrm{NaO}_{3} \mathrm{~S}\right]^{+}\left(\mathrm{M}+\mathrm{Na}^{+}\right)$625.1302, found 625.1308 .

\section{3-(trimethylamine-boranyl(4-methoxyphenyl)methyl)-1-((4-bromophenyl)sulfony} l)-4-(thiophen-2-yl)-1H-pyrrole (2p)

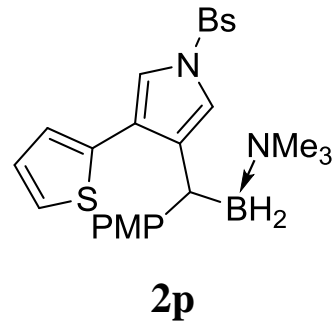

Compound 2p was prepared in $51 \%$ yield $(28.4 \mathrm{mg}$ ) according to the general procedure (Scheme 2, entry 16). The product was isolated through silica gel column chromatography $(\mathrm{PE}: \mathrm{EA}=5: 1)$ as a white solid $\left(\mathrm{mp} 129-130{ }^{\circ} \mathrm{C}\right) .{ }^{1} \mathrm{H}$ NMR $(400 \mathrm{MHz}$, $\left.\mathrm{CDCl}_{3}\right) \delta 7.70(\mathrm{~d}, J=8.8 \mathrm{~Hz}, 2 \mathrm{H}), 7.60(\mathrm{~d}, J=8.8 \mathrm{~Hz}, 2 \mathrm{H}), 7.21-7.19(\mathrm{~m}, 2 \mathrm{H}), 7.15$ $(\mathrm{d}, J=2.4 \mathrm{~Hz}, 1 \mathrm{H}), 7.02(\mathrm{~d}, J=8.4 \mathrm{~Hz}, 2 \mathrm{H}), 6.98(\mathrm{dd}, J=5.2,3.6 \mathrm{~Hz}, 1 \mathrm{H}), 6.93(\mathrm{dd}$, $J=3.6,1.2 \mathrm{~Hz}, 1 \mathrm{H}), 6.71(\mathrm{~d}, J=8.8 \mathrm{~Hz}, 2 \mathrm{H}), 3.74(\mathrm{~s}, 3 \mathrm{H}), 3.38(\mathrm{t}, J=4.4 \mathrm{~Hz}, 1 \mathrm{H})$, $2.36(\mathrm{~s}, 9 \mathrm{H}) ;{ }^{13} \mathrm{C} \mathrm{NMR}\left(100 \mathrm{MHz}, \mathrm{CDCl}_{3}\right) \delta 156.3,141.0,138.1,136.2,135.7,132.5$, 
128.9, 128.7, 128.2, 127.2, 125.4, 124.6, 124.3, 119.5, 118.1, 113.4, 55.2, 52.6, 33.0;

${ }^{11} \mathrm{~B}$ NMR (128 MHz, $\mathrm{CDCl}_{3}$ ) $\delta$ 3.61; IR (neat): 2919, 2379, 1573, 1506, 1372, 1272 , 1174, 1067, 744, $585 \mathrm{~cm}^{-1}$; HRESIMS Calcd for $\left[\mathrm{C}_{25} \mathrm{H}_{28} \mathrm{BBrN}_{2} \mathrm{NaO}_{3} \mathrm{~S}_{2}\right]^{+}\left(\mathrm{M}+\mathrm{Na}^{+}\right)$ 581.0710, found 581.0714.

3-(trimethylamine-boranyl(4-methoxyphenyl)methyl)-1-((4-bromophenyl)sulfony 1)-4-cyclopropyl-1H-pyrrole (2q)

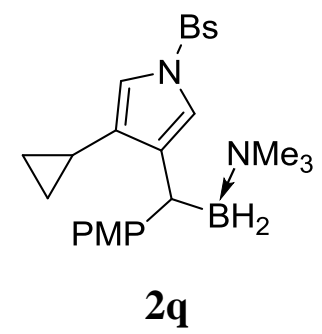

Compound 2q was prepared in $71 \%$ yield $(36.7 \mathrm{mg}$ ) according to the general procedure (Scheme 2, entry 17). The product was isolated through silica gel column chromatography $(\mathrm{PE}: \mathrm{EA}=5: 1)$ as a white solid $\left(\mathrm{mp} 164-165^{\circ} \mathrm{C}\right) .{ }^{1} \mathrm{H}$ NMR $(400 \mathrm{MHz}$, $\left.\mathrm{CDCl}_{3}\right) \delta 7.60(\mathrm{~d}, J=8.8 \mathrm{~Hz}, 2 \mathrm{H}), 7.55(\mathrm{~d}, J=8.8 \mathrm{~Hz}, 2 \mathrm{H}), 7.04-7.01(\mathrm{~m}, 3 \mathrm{H}), 6.70$ $(\mathrm{d}, J=8.4 \mathrm{~Hz}, 2 \mathrm{H}), 6.58(\mathrm{dd}, J=2.4,0.8 \mathrm{~Hz}, 1 \mathrm{H}), 3.74(\mathrm{~s}, 3 \mathrm{H}), 3.24(\mathrm{t}, J=4.8 \mathrm{~Hz}$, $1 \mathrm{H}), 2.42(\mathrm{~s}, 9 \mathrm{H}), 1.49-1.42(\mathrm{~m}, 1 \mathrm{H}), 0.78-0.71(\mathrm{~m}, 1 \mathrm{H}), 0.64-0.57(\mathrm{~m}, 1 \mathrm{H}), 0.36$ - $0.27(\mathrm{~m}, 2 \mathrm{H}) ;{ }^{13} \mathrm{C}$ NMR $\left(100 \mathrm{MHz}, \mathrm{CDCl}_{3}\right) \delta 156.3,141.4,138.5,138.4,133.8$, $132.2,129.0,128.1,127.9,118.6,115.9,113.3,55.2,52.6,33.1,7.0,6.5,6.1 ;{ }^{11} \mathrm{~B}$ NMR (128 MHz, $\left.\mathrm{CDCl}_{3}\right) \delta 2.67$; IR (neat): 2943, 2351, 1574, 1506, 1366, 1247, 1174 , 1069, 843, $745 \mathrm{~cm}^{-1}$; HRESIMS Calcd for $\left[\mathrm{C}_{24} \mathrm{H}_{30} \mathrm{BBrN}_{2} \mathrm{NaO}_{3} \mathrm{~S}\right]^{+}\left(\mathrm{M}+\mathrm{Na}^{+}\right)$ 539.1146, found 539.1143.

3-((4-(benzyloxy)phenyl)(trimethylamine-boranyl)methyl)-1-((4-bromophenyl)su Ifonyl)-4-phenyl-1H-pyrrole (2r)<smiles>CN(C)[C@H](c1ccc(OCc2ccccc2)cc1)c1cn([13CH3])cc1-c1ccccc1</smiles> 
Compound 2r was prepared in $69 \%$ yield $(43.3 \mathrm{mg}$ ) according to the general procedure (Scheme 2, entry 18). The product was isolated through silica gel column chromatography $(\mathrm{PE}: \mathrm{EA}=5: 1)$ as a white solid $\left(\mathrm{mp} 171-172{ }^{\circ} \mathrm{C}\right) .{ }^{1} \mathrm{H}$ NMR $(400 \mathrm{MHz}$, $\left.\mathrm{CDCl}_{3}\right) \delta 7.69(\mathrm{~d}, J=8.4 \mathrm{~Hz}, 2 \mathrm{H}), 7.58(\mathrm{~d}, J=8.4 \mathrm{~Hz}, 2 \mathrm{H}), 7.42(\mathrm{~d}, J=7.2 \mathrm{~Hz}, 2 \mathrm{H})$, $7.37(\mathrm{t}, J=7.2 \mathrm{~Hz}, 2 \mathrm{H}), 7.32-7.25(\mathrm{~m}, 4 \mathrm{H}), 7.23(\mathrm{~d}, J=6.8 \mathrm{~Hz}, 2 \mathrm{H}), 7.20(\mathrm{~d}, J=2.0$ $\mathrm{Hz}, 1 \mathrm{H}), 7.03-7.01(\mathrm{~m}, 3 \mathrm{H}), 6.80(\mathrm{~d}, J=8.4 \mathrm{~Hz}, 2 \mathrm{H}), 5.00(\mathrm{~s}, 2 \mathrm{H}), 3.19$ (t, $J=4.4$ $\mathrm{Hz}, 1 \mathrm{H}), 2.27$ (s, 9H); ${ }^{13} \mathrm{C} \mathrm{NMR}\left(100 \mathrm{MHz}, \mathrm{CDCl}_{3}\right) \delta 155.6,141.6,138.3,137.5$, $136.3,134.5,132.4,132.0,129.0,128.9,128.5,128.4,128.1,128.0,127.7,127.5$, 126.8, 119.0, 117.7, 114.4, 70.1, 52.5, 33.2; ${ }^{11} \mathrm{~B}$ NMR $\left(128 \mathrm{MHz}, \mathrm{CDCl}_{3}\right) \delta 3.24$; IR (neat): 2919, 2364, 1506, 1372, 1174, 1067, 701, $586 \mathrm{~cm}^{-1}$; HRESIMS Calcd for $\left[\mathrm{C}_{33} \mathrm{H}_{34} \mathrm{BBrN}_{2} \mathrm{NaO}_{3} \mathrm{~S}\right]^{+}\left(\mathrm{M}+\mathrm{Na}^{+}\right) 651.1459$, found 651.1455 .

\section{3-(benzo[d][1,3]dioxol-5-yl(trimethylamine-boranyl)methyl)-1-((4-bromophenyl)} sulfonyl)-4-phenyl-1H-pyrrole (2s)

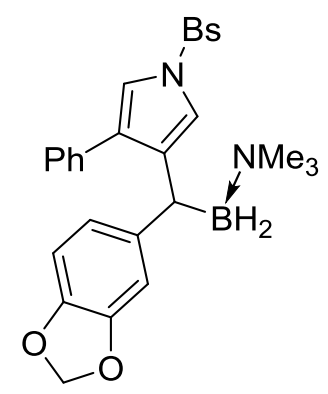

$2 s$

Compound $2 \mathrm{~s}$ was prepared in $45 \%$ yield $(25.4 \mathrm{mg}$ ) according to the general procedure (Scheme 2, entry 19). The product was isolated through silica gel column chromatography (PE:EA = 5:1) as a white solid (mp 144-145 $\left.{ }^{\circ} \mathrm{C}\right) .{ }^{1} \mathrm{H}$ NMR (400 MHz, $\left.\mathrm{CDCl}_{3}\right) \delta 7.70(\mathrm{~d}, J=8.8 \mathrm{~Hz}, 2 \mathrm{H}), 7.59(\mathrm{~d}, J=8.8 \mathrm{~Hz}, 2 \mathrm{H}), 7.31-7.19(\mathrm{~m}, 6 \mathrm{H}), 7.03$ $(\mathrm{d}, J=2.4 \mathrm{~Hz}, 1 \mathrm{H}), 6.68(\mathrm{~d}, J=1.6 \mathrm{~Hz}, 1 \mathrm{H}), 6.60(\mathrm{~d}, J=8.0 \mathrm{~Hz}, 1 \mathrm{H}), 6.50(\mathrm{dd}, J=$ 8.0, 1.6 Hz, 1H), $5.86(\mathrm{dd}, J=3.6,1.6 \mathrm{~Hz}, 2 \mathrm{H}), 3.17$ (t, $J=4.4 \mathrm{~Hz}, 1 \mathrm{H}), 2.29$ (s, 9H); ${ }^{13} \mathrm{C} \mathrm{NMR}\left(100 \mathrm{MHz}, \mathrm{CDCl}_{3}\right) \delta 147.3,144.0,143.4,138.2,136.1,134.5,132.5,132.0$, 129.0, 128.6, 128.1(2), 128.0(7), 126.8, 120.5, 119.1, 117.8, 108.8, 107.6, 100.4, 52.5, 
33.8; ${ }^{11} \mathrm{~B}$ NMR (128 MHz, $\left.\mathrm{CDCl}_{3}\right) \delta$ 3.17; IR (neat): 2918, 2351, 1574, 1390, 1240 , 1174, 1067, 744, $586 \mathrm{~cm}^{-1}$; HRESIMS Calcd for $\left[\mathrm{C}_{27} \mathrm{H}_{28} \mathrm{BBrN}_{2} \mathrm{NaO}_{4} \mathrm{~S}\right]^{+}\left(\mathrm{M}+\mathrm{Na}^{+}\right)$ 589.0938, found 589.0934.

\section{4-(trimethylamine-boranyl(1-((4-bromophenyl)sulfonyl)-4-phenyl-1H-pyrrol-3-yl} )methyl)- $N, N$-dimethylaniline (2t)

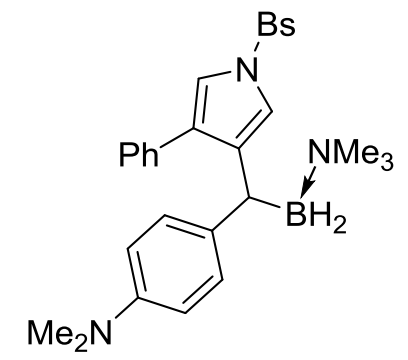

$2 \mathrm{t}$

Compound $\mathbf{2 t}$ was prepared in $63 \%$ yield $(35.4 \mathrm{mg}$ ) according to the general procedure (Scheme 2, entry 20). The product was isolated through silica gel column chromatography (PE:EA $=5: 1)$ as a yellow solid $\left(\mathrm{mp} 121-122{ }^{\circ} \mathrm{C}\right) .{ }^{1} \mathrm{H}$ NMR (400 $\left.\mathrm{MHz} \mathrm{CDCl}_{3}\right) \delta 7.68(\mathrm{~d}, J=8.8 \mathrm{~Hz}, 2 \mathrm{H}), 7.58(\mathrm{~d}, J=8.8 \mathrm{~Hz}, 2 \mathrm{H}), 7.33-7.26(\mathrm{~m}, 5 \mathrm{H})$, $7.18(\mathrm{~d}, J=2.4 \mathrm{~Hz}, 1 \mathrm{H}), 7.02-6.99(\mathrm{~m}, 3 \mathrm{H}), 6.63(\mathrm{~d}, J=8.4 \mathrm{~Hz}, 2 \mathrm{H}), 3.15(\mathrm{t}, J=4.4$ $\mathrm{Hz}, 1 \mathrm{H}), 2.87$ (s, 6H), 2.27 (s, 9H); $\left.{ }^{13} \mathrm{C} \mathrm{NMR} \mathrm{(100} \mathrm{MHz,} \mathrm{CDCl}_{3}\right) \delta$ 147.8, 138.4, $137.8,136.8,134.7,132.4,132.1,129.1,128.7,128.4,128.1,128.0,126.7,119.0$, 117.6, 113.4, 52.4, 41.2, 33.0; ${ }^{11} \mathrm{~B}$ NMR (128 MHz, $\mathrm{CDCl}_{3}$ ) $\delta$ 3.78; IR (neat): 2919, 2355, 1514, 1370, 1174, 1067, 745, 630, $589 \mathrm{~cm}^{-1}$; HRESIMS Calcd for $\left[\mathrm{C}_{28} \mathrm{H}_{33} \mathrm{BBrN}_{3} \mathrm{NaO}_{2} \mathrm{~S}\right]^{+}\left(\mathrm{M}+\mathrm{Na}^{+}\right)$588.1462, found 588.1466.

\subsection{Preparative-scale synthesis of $2 a$}
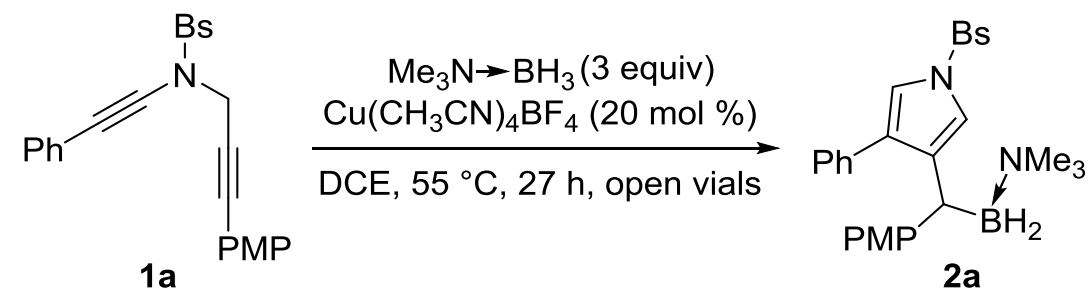

$\mathrm{Cu}\left(\mathrm{CH}_{3} \mathrm{CN}\right)_{4} \mathrm{BF}_{4}(62.9 \mathrm{mg}, 0.2 \mathrm{mmol})$ was added to a solution of the $N$-propargyl ynamide 1a (480.4 mg, $1 \mathrm{mmol})$ and trimethylamine borane $(218.9 \mathrm{mg}, 3 \mathrm{mmol})$ in 
DCE $(10 \mathrm{~mL})$ in a $25 \mathrm{~mL}$ eggplant-shaped bottle at room temperature. The reaction mixture was stirred at $55{ }^{\circ} \mathrm{C}$ without being sealed for $27 \mathrm{~h}$. Upon completion, the mixture was then concentrated and the residue was purified by chromatography on silicagel (PE:EA = 5:1) to afford the desired organoboron compound 2a (384.0 mg, $69 \%$ yield).

\subsection{Transformations}

1-((4-bromophenyl)sulfonyl)-3-((4-methoxyphenyl)(4,4,5,5-tetramethyl-1,3,2-diox aborolan-2-yl)methyl)-4-phenyl-1H-pyrrole (3) ${ }^{3}$

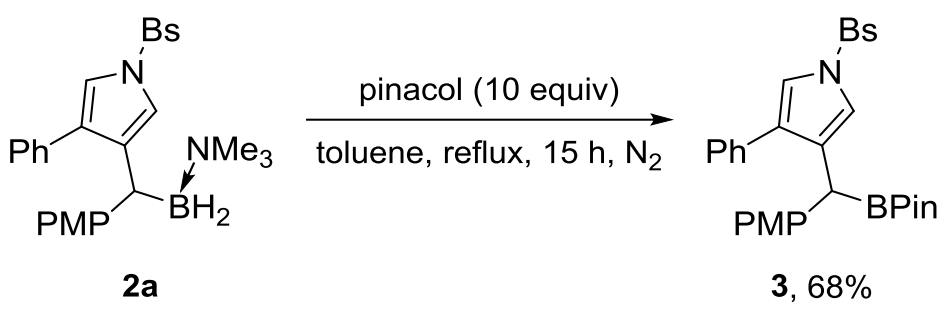

To a solution of $\mathbf{2 a}(100.0 \mathrm{mg}, 0.18 \mathrm{mmol})$ in toluene ( $2 \mathrm{~mL})$, pinacol (anhydrous, 212 $\mathrm{mg}, 1.8 \mathrm{mmol}$ ) was added under nitrogen atmosphere. The resulting mixture was heated to reflux for $15 \mathrm{~h}$. After concentration in vacuo, the residue was chromatographied on silica gel (PE:EA $=10: 1)$ to give $3(74.7 \mathrm{mg})$ as a white solid (mp 101-102 ${ }^{\circ} \mathrm{C}$ ). Yield: 68\%. ${ }^{1} \mathrm{H}$ NMR $\left(400 \mathrm{MHz}, \mathrm{CDCl}_{3}\right) \delta 7.69(\mathrm{~d}, J=8.8 \mathrm{~Hz}, 2 \mathrm{H})$, $7.60(\mathrm{~d}, J=8.4 \mathrm{~Hz}, 2 \mathrm{H}), 7.30-7.22(\mathrm{~m}, 5 \mathrm{H}), 7.14(\mathrm{~d}, J=2.4 \mathrm{~Hz}, 1 \mathrm{H}), 7.04(\mathrm{~d}, J=$ $8.8 \mathrm{~Hz}, 2 \mathrm{H}), 6.98(\mathrm{dd}, J=2.4,0.8 \mathrm{~Hz}, 1 \mathrm{H}), 6.79(\mathrm{~d}, J=8.8 \mathrm{~Hz}, 2 \mathrm{H}), 3.78(\mathrm{~s}, 3 \mathrm{H})$, $3.69(\mathrm{~s}, 1 \mathrm{H}), 1.02(\mathrm{~d}, J=11.6 \mathrm{~Hz}, 12 \mathrm{H}) ;{ }^{13} \mathrm{C} \mathrm{NMR}\left(100 \mathrm{MHz}, \mathrm{CDCl}_{3}\right) \delta 157.7,138.1$, $133.7,132.5,131.2,129.4,129.2,128.8,128.5,128.4,128.2,127.1,119.9,118.4$, 113.9, 83.6, 55.1, 28.5, 24.4, 24.3; ${ }^{11} \mathrm{~B}$ NMR (128 MHz, $\left.\mathrm{CDCl}_{3}\right) \delta 41.99$; IR (neat): 2977, 1647, 1509, 1375, 1256, 1176, 1067, 745, $509 \mathrm{~cm}^{-1}$; HRESIMS Calcd for $\left[\mathrm{C}_{30} \mathrm{H}_{31} \mathrm{BBrNNaO}_{5} \mathrm{~S}\right]^{+}\left(\mathrm{M}+\mathrm{Na}^{+}\right)$630.1092, found 630.1099.

2-((1-((4-bromophenyl)sulfonyl)-4-phenyl-1H-pyrrol-3-yl)(4-methoxyphenyl)met hyl)-6-methyl-1,3,6,2-dioxazaborocane-4,8-dione $(4)^{3}$ 


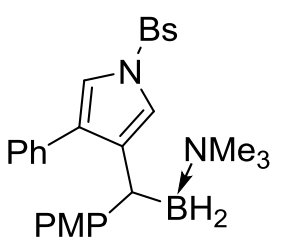

$2 a$

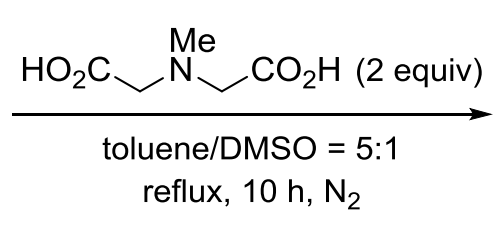

$4,79 \%$

$10 \mathrm{~mL}$ Schlenk tube was charged with 2a (138.0 $\mathrm{mg}, 0.25 \mathrm{mmol})$, $N$-methyliminodiacetic acid $(73.5 \mathrm{mg}, \quad 0.5 \mathrm{mmol})$. Then $2.5 \mathrm{~mL}$ solvent (toluene/DMSO = 5:1) was injected under nitrogen atmosphere. The resulting mixture was heated to reflux for $10 \mathrm{~h}$. After concentration in vacuo, the residue was chromatographied on silica gel (PE:EA $=1: 1)$ to give $4(125.6 \mathrm{mg})$ as a white solid (mp 154-155 $\left.{ }^{\circ} \mathrm{C}\right)$. Yield: $79 \% .{ }^{1} \mathrm{H}$ NMR $\left(400 \mathrm{MHz}, \mathrm{CDCl}_{3}\right) \delta 7.74(\mathrm{~d}, J=8.8 \mathrm{~Hz}, 2 \mathrm{H})$, $7.62(\mathrm{~d}, J=8.8 \mathrm{~Hz}, 2 \mathrm{H}), 7.40(\mathrm{~d}, J=2.0 \mathrm{~Hz}, 1 \mathrm{H}), 7.34-7.28(\mathrm{~m}, 3 \mathrm{H}), 7.13-7.11(\mathrm{~m}$, 2H), $7.07-7.04(\mathrm{~m}, 3 \mathrm{H}), 6.72(\mathrm{~d}, J=8.4 \mathrm{~Hz}, 2 \mathrm{H}), 3.78-3.69(\mathrm{~m}, 5 \mathrm{H}), 3.28-3.24$ (m, 2H), $3.04(\mathrm{~d}, J=16.8 \mathrm{~Hz}, 1 \mathrm{H}), 2.42(\mathrm{~s}, 3 \mathrm{H}) ;{ }^{13} \mathrm{C} \mathrm{NMR}\left(100 \mathrm{MHz}, \mathrm{CDCl}_{3}\right) \delta 168.0$, $167.3,157.6,137.7,133.5,133.2,132.8,131.7,129.7,129.4,129.2,129.1,128.5$, $128.4,127.4,118.9,117.9,113.9,62.9,62.6,55.2,45.5 ;{ }^{11} \mathrm{~B} \mathrm{NMR}\left(128 \mathrm{MHz}, \mathrm{CDCl}_{3}\right)$ $\delta$ 14.18; IR (neat): 2927, 1770, 1508, 1300, 1251, 1176, 1069, 745, $509 \mathrm{~cm}^{-1}$; HRESIMS Calcd for $\left[\mathrm{C}_{29} \mathrm{H}_{26} \mathrm{BBrN}_{2} \mathrm{NaO}_{7} \mathrm{~S}\right]^{+}\left(\mathrm{M}+\mathrm{Na}^{+}\right) 659.0629$, found 659.0632 .

\section{(1-((4-bromophenyl)sulfonyl)-4-phenyl-1H-pyrrol-3-yl)(4-methoxyphenyl)metha} $\operatorname{nol}(5)^{3}$<smiles></smiles>

$2 \mathbf{a}$<smiles>OC(N=[W])c1cn(S)cc1-c1ccccc1</smiles>

$5,82 \%$

To a solution of $\mathbf{2 a}(110.6 \mathrm{mg}, 0.2 \mathrm{mmol})$ in $\mathrm{MeOH}(2 \mathrm{~mL}), 30 \% \mathrm{H}_{2} \mathrm{O}_{2}(0.41 \mathrm{~mL}, 4$ mmol) was added dropwise. The resulting mixture was heated reflux for $5 \mathrm{~h}$. After concentration in vacuo, the residue was chromatographied on silicagel (PE:DCM = 1:1) to give $5(81.2 \mathrm{mg})$ as a colorless oil. Yield: $82 \% .{ }^{1} \mathrm{H}$ NMR $\left(400 \mathrm{MHz}, \mathrm{CDCl}_{3}\right) \delta$ $7.71(\mathrm{~d}, J=8.8 \mathrm{~Hz}, 2 \mathrm{H}), 7.62(\mathrm{~d}, J=8.8 \mathrm{~Hz}, 2 \mathrm{H}), 7.30-7.25(\mathrm{~m}, 5 \mathrm{H}), 7.16-7.14(\mathrm{~m}$, 3H), $7.01(\mathrm{dd}, J=2.4,0.4 \mathrm{~Hz}, 1 \mathrm{H}), 6.80(\mathrm{~d}, J=8.8 \mathrm{~Hz}, 2 \mathrm{H}), 5.75(\mathrm{~s}, 1 \mathrm{H}), 3.77(\mathrm{~s}, 3 \mathrm{H})$, 
$2.20(\mathrm{~s}, 1 \mathrm{H}) ;{ }^{13} \mathrm{C}$ NMR $\left(100 \mathrm{MHz}, \mathrm{CDCl}_{3}\right) \delta 159.1,137.7,134.7,133.1,132.8,131.6$, 129.6, 129.2, 128.3(8), 128.3(7), 127.9, 127.3, 119.5, 118.6, 113.8, 69.0, 55.2; IR (neat): 3432 (br), 2836, 1610, 1511, 1374, 1250, 1068, 745, 635, $589 \mathrm{~cm}^{-1}$; HRESIMS Calcd for $\left[\mathrm{C}_{24} \mathrm{H}_{20} \mathrm{BrNNaO}_{4} \mathrm{~S}\right]^{+}\left(\mathrm{M}+\mathrm{Na}^{+}\right)$520.0189, found 520.0184.

\section{Reference:}

1. Hong, F.-L.; Wang, Z.-S.; Wei, D.-D.; Zhai, T.-Y.; Deng, G.-C.; Lu, X.; Liu, R.-S.; Ye, L.-W. Generation of Donor/Donor Copper Carbenes through Copper-Catalyzed Diyne Cyclization: Enantioselective and Divergent Synthesis of Chiral Polycyclic Pyrroles. J. Am. Chem. Soc. 2019, 141, 16961.

2. Zhu, X.-Q.; Hong, P.; Zeng, Y.-X.; Zhen, Y.-Y.; Hong, F.-L.; Lu, X.; Ye, L.-W. Copper-Catalyzed Asymmetric Cyclization of Alkenyl Diynes: Method Development and New mechanistic Insights. Chem. Sci. 2021, 11, 9466.

3. Yang, J.-M.; Li, Z.-Q.; Li, M.-L.; He, Q.; Zhu, S.-F.; Zhou, Q.-L. Catalytic B-H Bond Insertion Reactions Using Alkynes as Carbene Precursors. J. Am. Chem. Soc. 2017, 139, 3784 . 


\section{Crystal data and structure refinement for $2 \mathrm{~s}$. CCDC Number $=2104195$.}

ORTEP drawing of $2 \mathrm{~s}$ (thermal ellipsoids set at $50 \%$ probability). Recrystallization from $n$-hexane/DCM afforded the single crystal suitable for X-ray diffraction analysis.
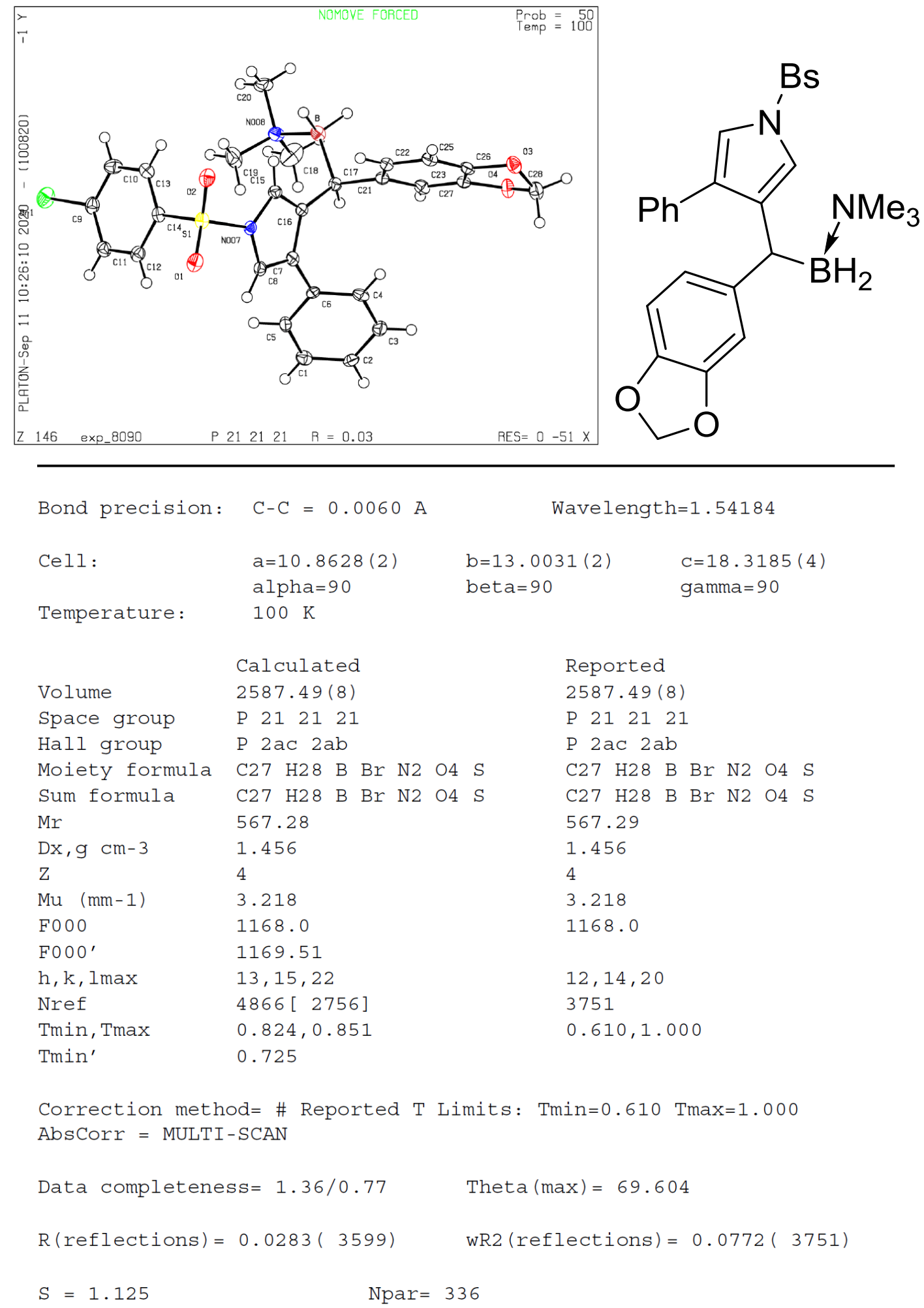

$\begin{array}{lll} & \text { Calculated } & \text { Reported } \\ \text { Volume } & 2587.49(8) & 2587.49(8) \\ \text { Space group } & \text { P 21 21 21 } & \text { P 21 21 21 } \\ \text { Hall group } & \text { P 2aC 2ab } & \text { P 2ac 2ab } \\ \text { Moiety formula } & \text { C27 H28 B Br N2 O4 S } & \text { C27 H28 B Br N2 O4 S } \\ \text { Sum formula } & \text { C27 H28 B Br N2 O4 S } & \text { C27 H28 B Br N2 O4 S } \\ \text { Mr } & 567.28 & 567.29 \\ \text { Dx, gm-3 } & 1.456 & 1.456 \\ \text { Z } & 4 & 4 \\ \text { Mu (mm-1) } & 3.218 & 3.218 \\ \text { F000 } & 1168.0 & 1168.0 \\ \text { F000' } & 1169.51 & \\ \text { h, k, lmax } & 13,15,22 & 12,14,20 \\ \text { Nref } & 4866[2756] & 3751 \\ \text { Tmin, Tmax } & 0.824,0.851 & 0.610,1.000 \\ \text { Tmin' } & 0.725 & \end{array}$

Correction method= \# Reported T Limits: Tmin=0.610 Tmax=1.000 AbsCorr = MULTI-SCAN

Data completeness $=1.36 / 0.77 \quad$ Theta $(\max )=69.604$

$\mathrm{R}($ reflections $)=0.0283(3599) \quad$ wR2 (reflections $)=0.0772(3751)$
Npar $=336$

$S=1.125$ 


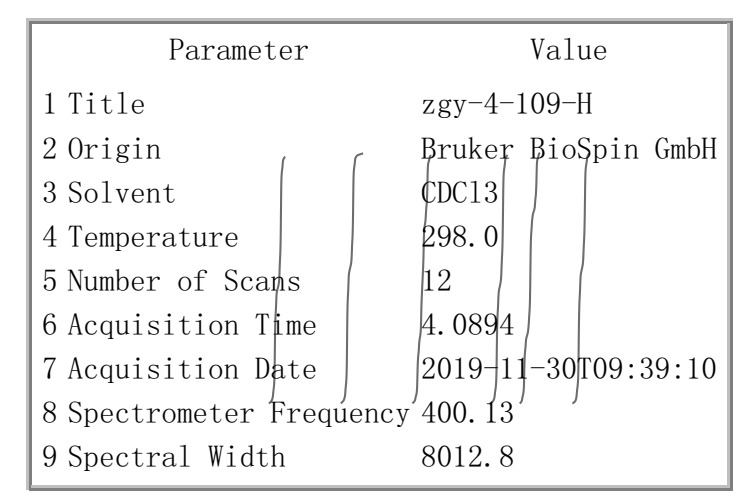
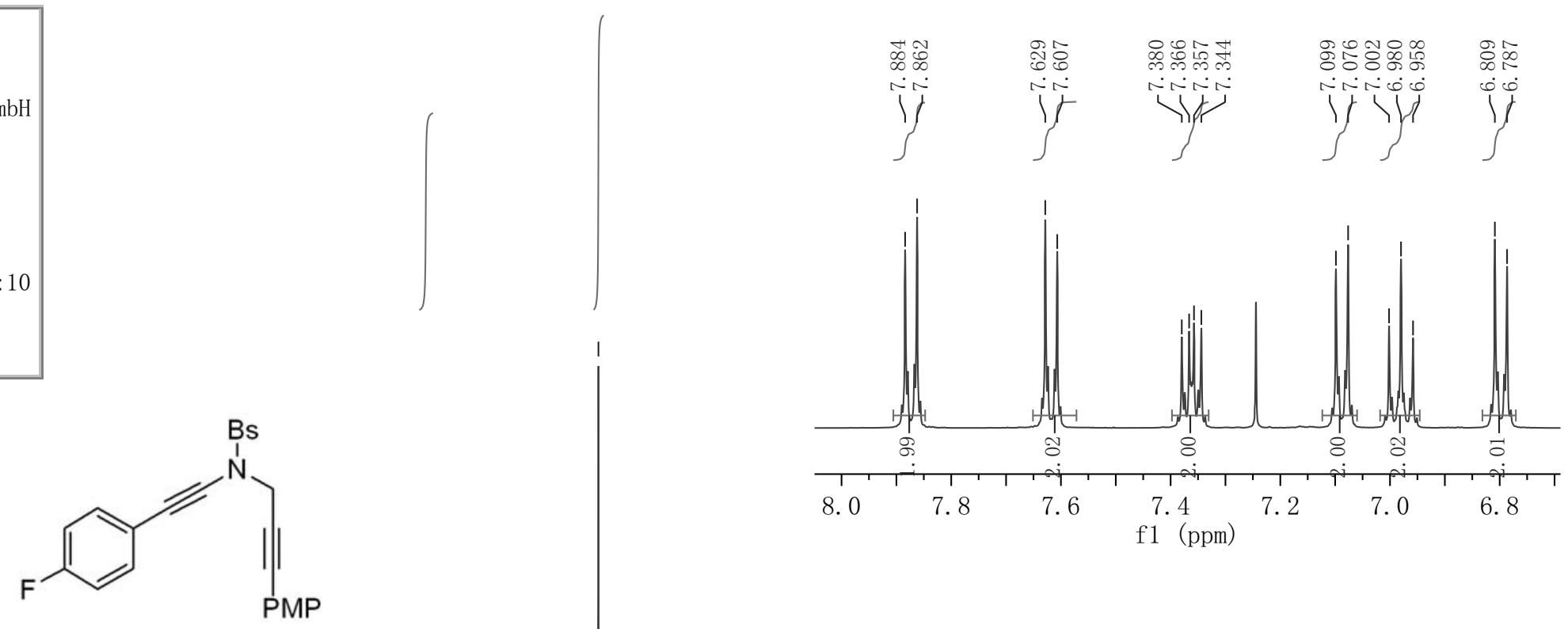

1f

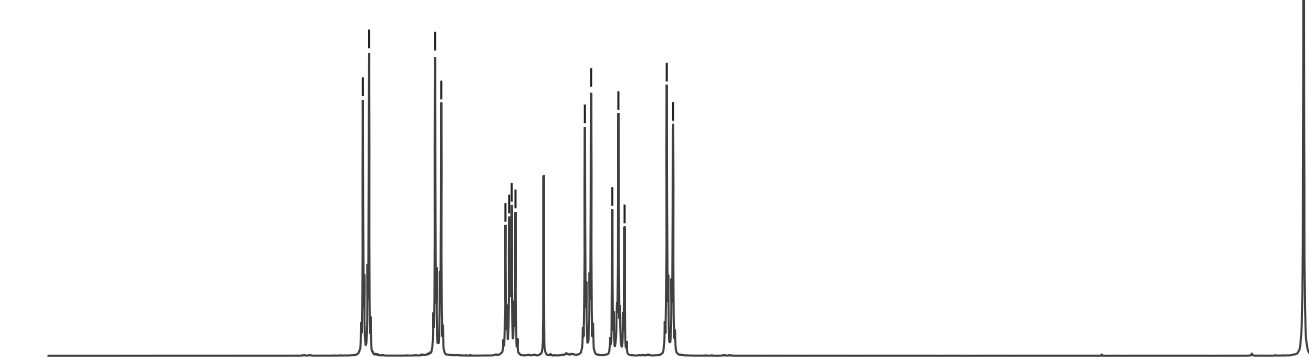

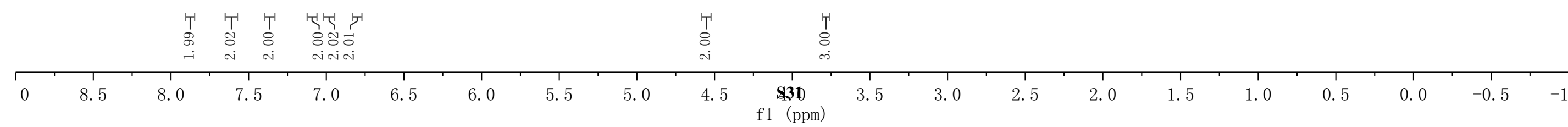




\begin{tabular}{|ll|}
\hline \multicolumn{1}{|c}{ Parameter } & \multicolumn{1}{c}{ Value } \\
1 Title & zgy-4-109-C \\
2 Origin & Bruker BioSpin GmbH \\
3 Solvent & 300.0 \\
4 Temperature & 31 \\
5 Number of Scans & 1.3631 \\
6 Acquisition Time & $2019-11-30 \mathrm{~T} 09: 44: 20$ \\
7 Acquisition Date & 24038.5 \\
8 Spectrometer Frequency & 100.61 \\
9 Spectral Width &
\end{tabular}
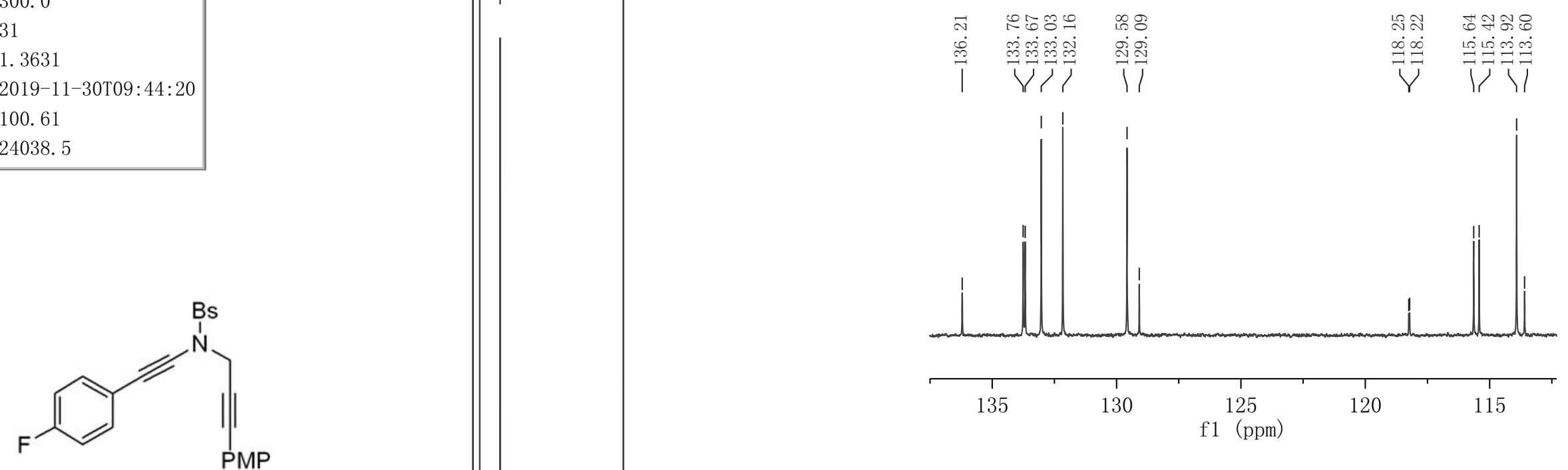

135

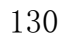

125

f1 (ppm)

120

115

$1 f$

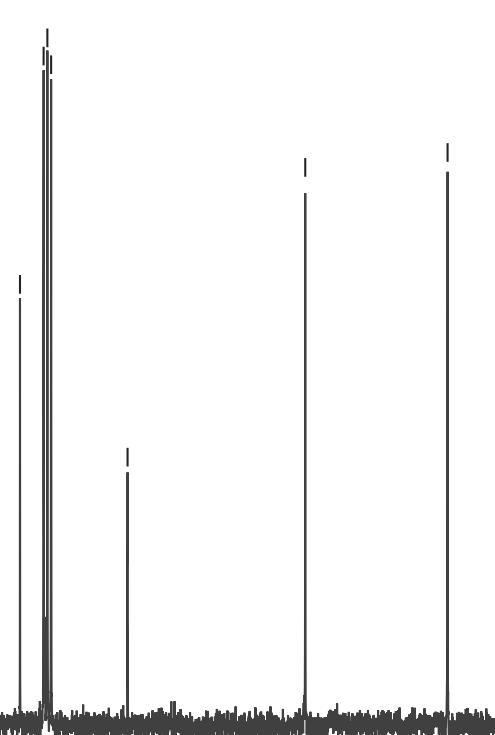

90

1
90

70

1
00

50

50

40




\begin{tabular}{|ll|}
\hline \multicolumn{1}{|c|}{ Parameter } & \multicolumn{1}{c|}{ Value } \\
1 Title & zgy-4-109-F \\
2 Origin & Bruker BioSpin GmbH \\
3 Solvent & CDC13 \\
4 Temperature & 297.3 \\
5 Number of Scans & 16 \\
6 Acquisition Time & 0.7340 \\
7 Acquisition Date & $2021-08-27 T 18: 01: 26$ \\
8 Spectrometer Frequency & 376.31 \\
9 Spectral Width & 89285.7 \\
\hline
\end{tabular}

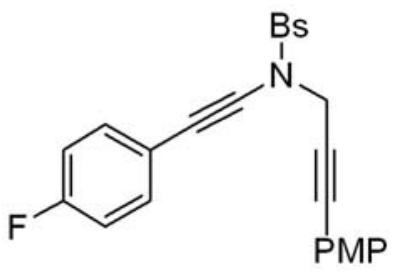

$1 f$

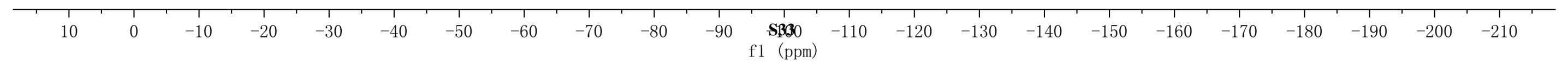




\begin{tabular}{|ll|}
\hline \multicolumn{1}{|c|}{ Parameter } & \multicolumn{1}{c|}{ Value } \\
1 Title & zgy-4-110-C \\
2 Origin & Bruker BioSpin GmbH \\
3 Solvent & CDC13 \\
4 Temperature & 300.0 \\
5 Number of Scans & 38 \\
6 Acquisition Time & 1.3631 \\
7 Acquisition Date & $2019-12-03$ T10:26:19 \\
8 Spectrometer Frequency 100.61 \\
9 Spectral Width & 24038.5 \\
\hline
\end{tabular}
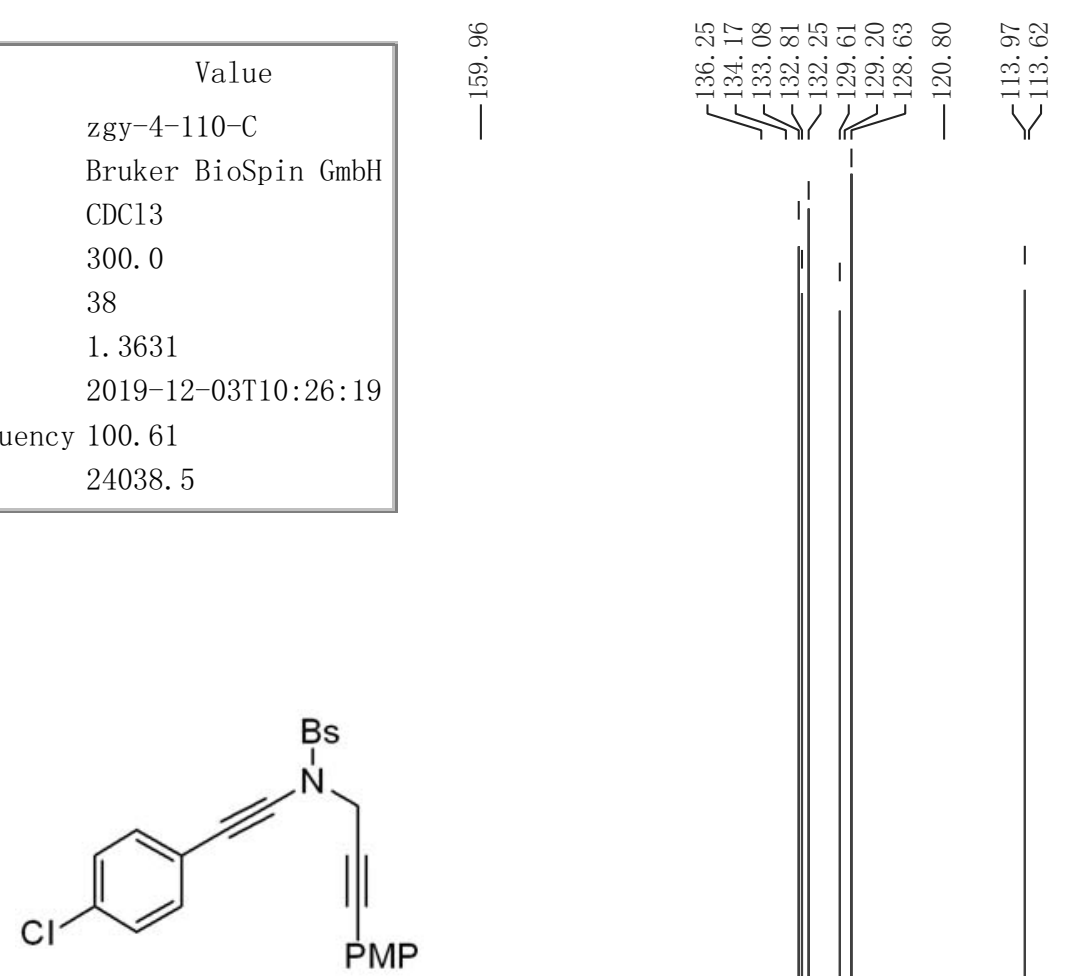

$1 \mathrm{~g}$
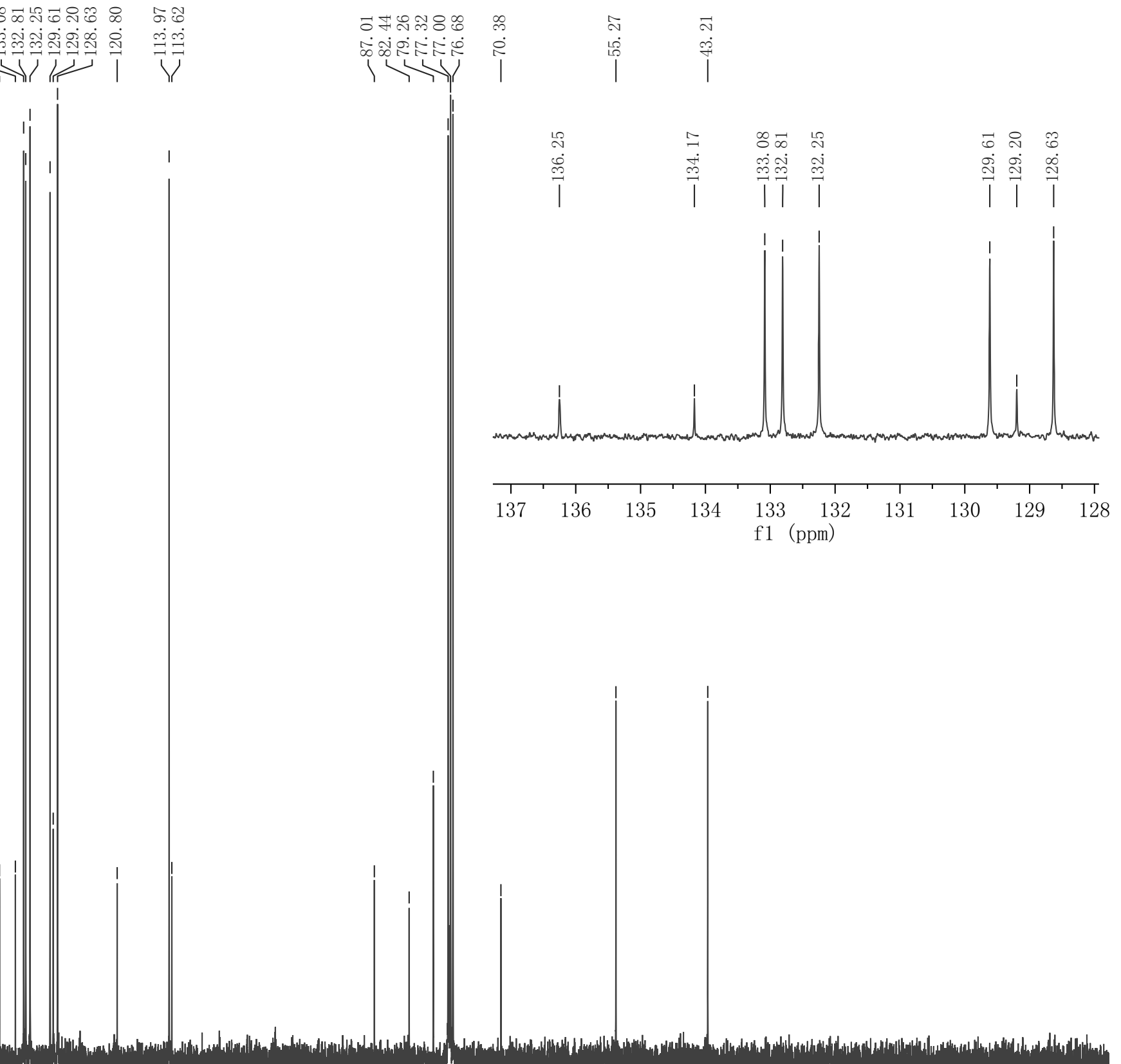

M.

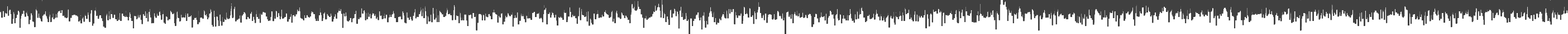

\begin{tabular}{|c|c|c|c|c|c|c|c|c|c|c|c|c|c|c|c|c|c|c|c|c|c|c|c|c|c|c|c|c|c|c|c|}
\hline & 210 & 200 & $\begin{array}{l}1 \\
190\end{array}$ & 180 & 170 & \multicolumn{2}{|r|}{160} & $\begin{array}{l}1 \\
150\end{array}$ & $\begin{array}{r} \\
14\end{array}$ & & 30 & 120 & S3D & 100 & 90 & \multicolumn{2}{|c|}{80} & 70 & 70 & & 60 & & 50 & \multicolumn{2}{|c|}{40} & \multicolumn{2}{|c|}{30} & \multicolumn{2}{|c|}{20} & & 10 \\
\hline
\end{tabular}




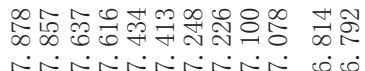

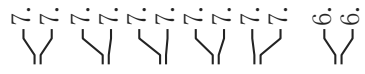

\begin{tabular}{|ll|}
\hline \multicolumn{1}{|c|}{ Parameter } & \multicolumn{1}{c|}{ Value } \\
1 Title & zgy-4-115-H \\
2 Origin & Bruker BioSpin GmbH \\
3 Solvent & GDCl3 \\
4 Temperature & 298.0 \\
5 Number of Scans & 30 \\
6 Acquisition Time & 4.0894 \\
7 Acquisition Date & $2019-12-04 T 10: 30: 22$ \\
8 Spectrometer Fryequency 400.13 & \\
9 Spectral Width & 8012.8 \\
\hline
\end{tabular}

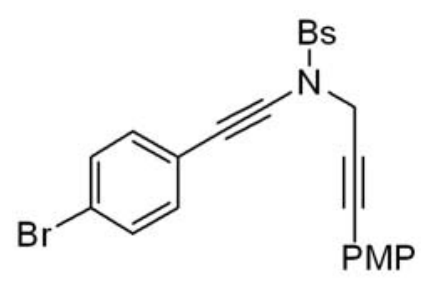

1h
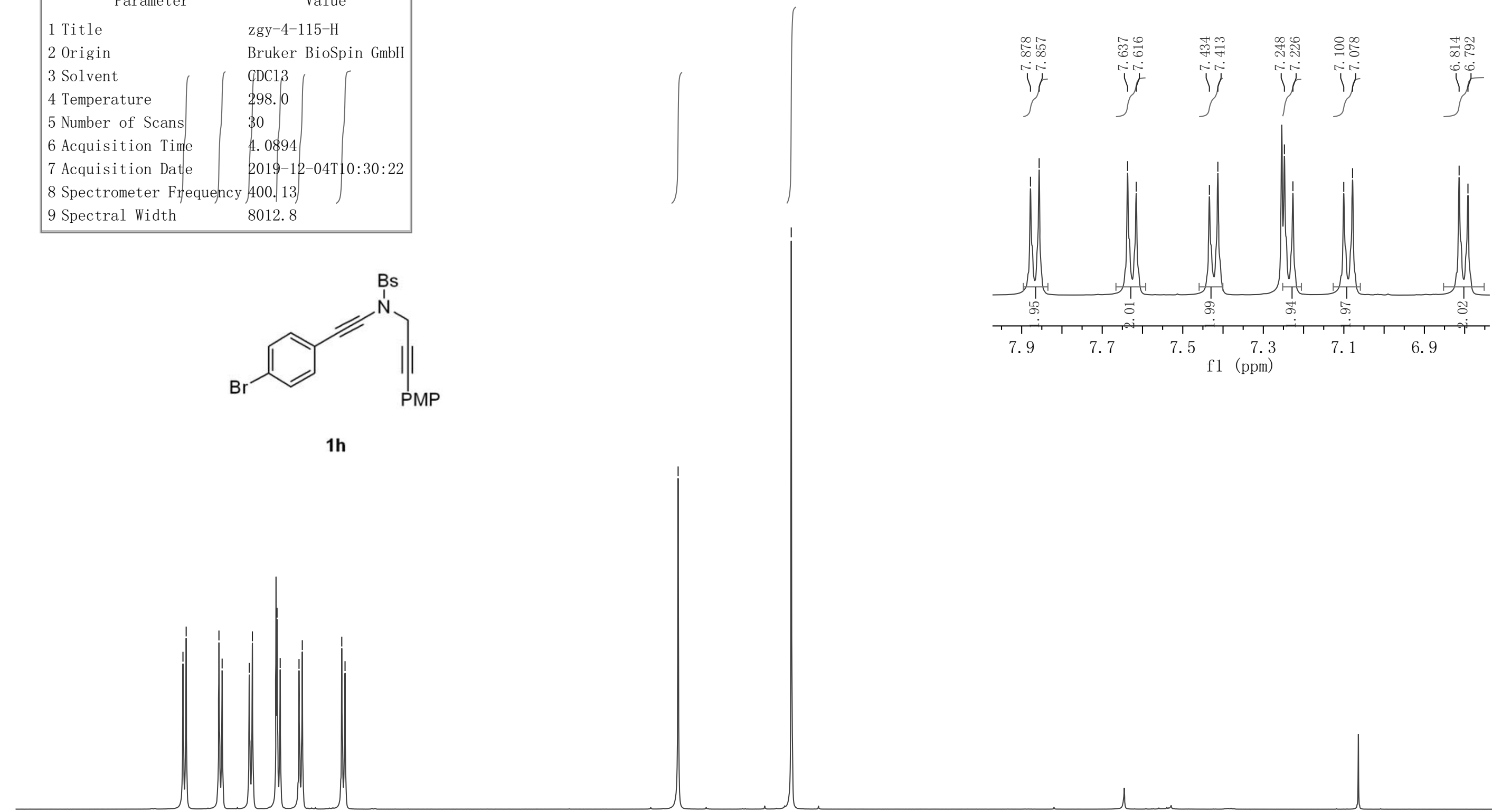

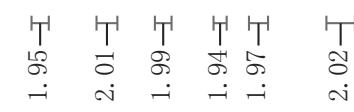
$\begin{array}{ll}\text { T' } & \text { T' } \\ 8 & 8 \\ \dot{4} & \dot{8}\end{array}$

$8.0 \quad 7.5 \quad 7.0$

6.5

6. $0 \quad 5.5$

$3.0 \quad 2.5$




\begin{tabular}{|ll|}
\hline \multicolumn{1}{|c|}{ Parameter } & \multicolumn{1}{c|}{ Value } \\
1 Title & zgy-4-115-C \\
2 Origin & Bruker BioSpin GmbH \\
3 Solvent & CDC13 \\
4 Temperature & 300.0 \\
5 Number of Scans & 80 \\
6 Acquisition Time & 1.3631 \\
7 Acquisition Date & $2019-12-04 \mathrm{~T} 10: 35: 02$ \\
8 Spectrometer Frequency 100.61 \\
9 Spectral Width & 24038.5 \\
\hline
\end{tabular}
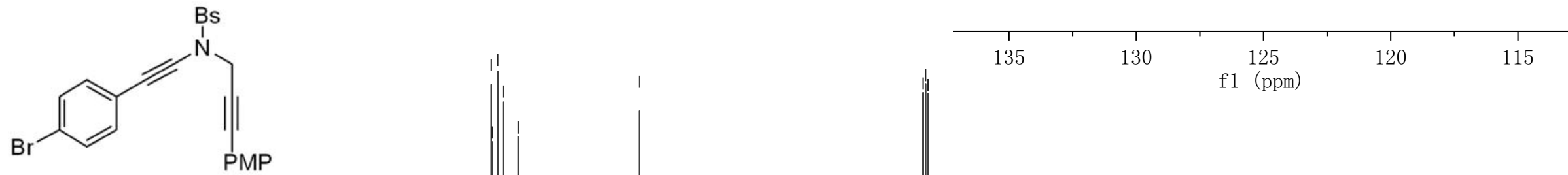

1h
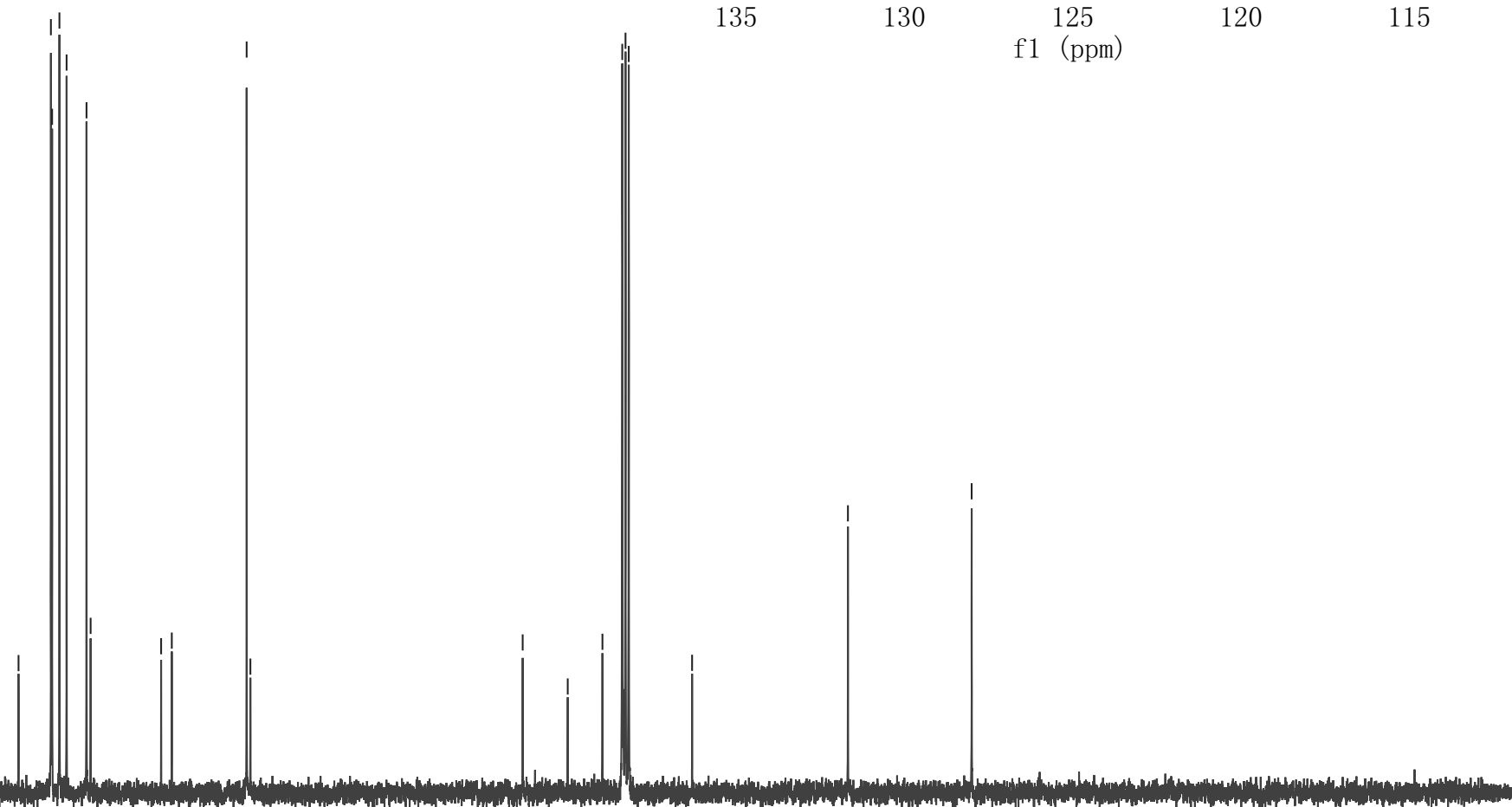

90

80

$80 \quad 70$

60

$60 \quad 50$

$50 \quad 40$

$\begin{array}{llllll}1 & 1 & 1 & 1 & 1 \\ 40 & 30 & 20 & 10 & 0\end{array}$




\begin{tabular}{|c|c|}
\hline Parameter & Value \\
\hline 1 Title & zgy $-4-116-H$ \\
\hline 2 Origin & Bruker Biospin GmbH \\
\hline 3 Solvent & $\mathrm{dDCl} 3$ \\
\hline 4 Temperature & 298.0 \\
\hline 5 Number of Scans & 15 \\
\hline 6 Acquisition time & 4. 0894 \\
\hline 7 Acquisition Date & 2019-12-04T17:39:03 \\
\hline \multicolumn{2}{|c|}{8 Spectrometer Frequency 400. 13} \\
\hline 9 Spectral Width & 8012.8 \\
\hline
\end{tabular}
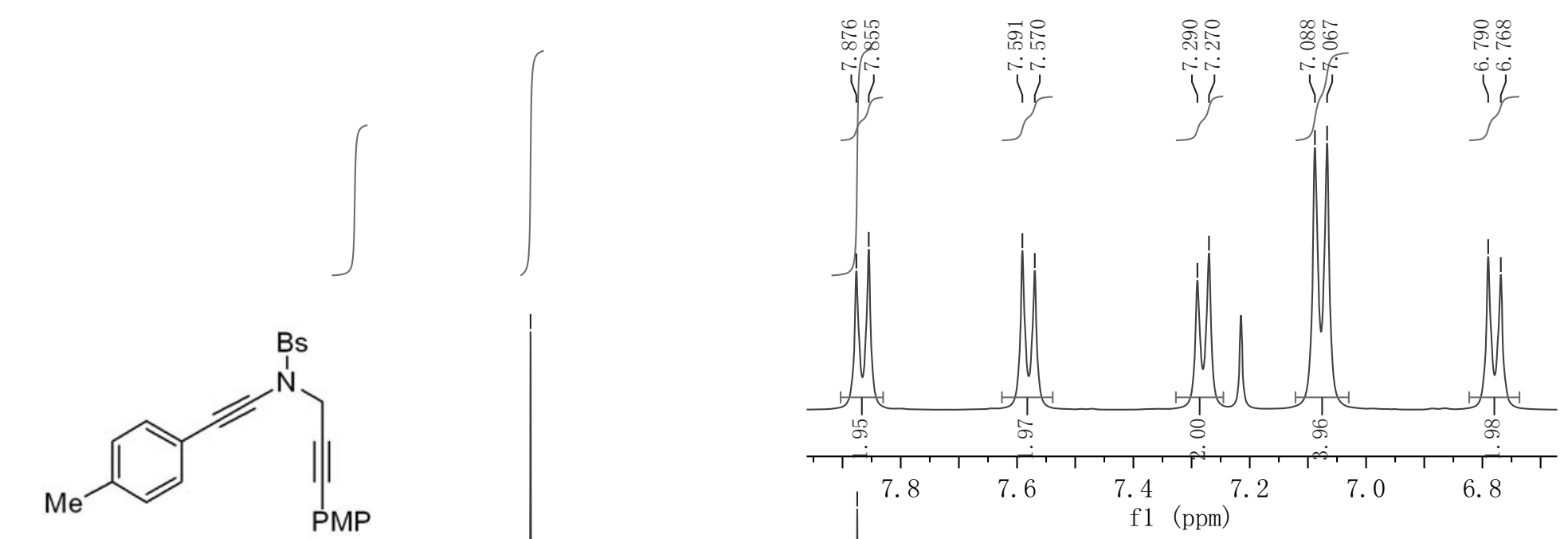

$1 i$

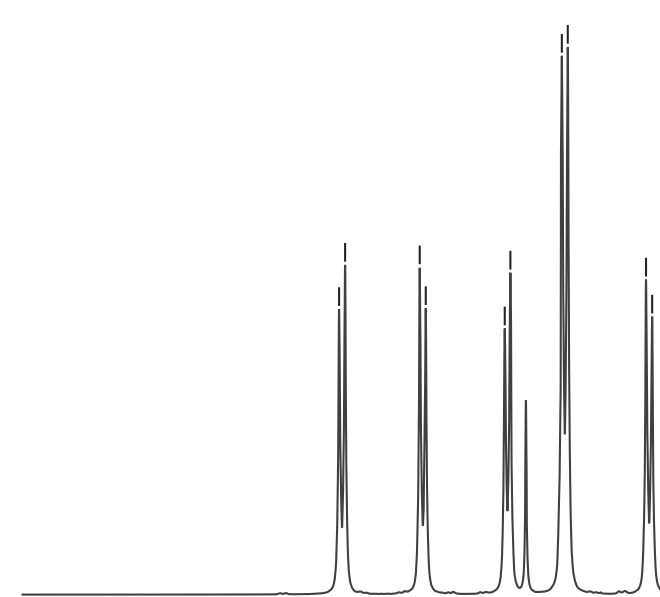

7.8

f1 (ppm)

6. 8

\begin{tabular}{|c|c|c|c|c|c|c|c|c|c|c|c|c|c|c|c|c|c|c|c|c|}
\hline & & 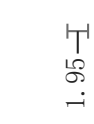 & 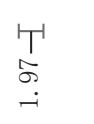 & 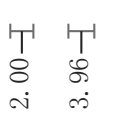 & $\begin{array}{l}\text { T1 } \\
\infty \\
\infty \\
-1\end{array}$ & & & & 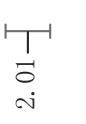 & $\begin{array}{l}\text { h } \\
c \\
c\end{array}$ & & & $\begin{array}{l}{ }_{1}^{1} \\
\delta \\
\dot{\alpha} \\
\dot{\infty}\end{array}$ & & & & & & & \\
\hline 0 & 8.5 & 8.0 & 7.5 & 7.0 & 6.5 & 6.0 & 5.5 & 5.0 & 4.5 & $\begin{array}{c}\mathbf{9 3 8} \\
\mathrm{f} 1 \text { (ppm) }\end{array}$ & 3.5 & 3.0 & 2.5 & 2.0 & 1.5 & 1. 0 & 0.5 & 0.0 & -0.5 & -1 \\
\hline
\end{tabular}




\begin{tabular}{|ll|}
\hline \multicolumn{1}{|c|}{ Parameter } & \multicolumn{1}{c|}{ Value } \\
1 Title & zgy-4-116-C \\
2 Origin & Bruker BioSpin GmbH \\
3 Solvent & CDC13 \\
4 Temperature & 300.0 \\
5 Number of Scans & 34 \\
6 Acquisition Time & 1.3631 \\
7 Acquisition Date & $2019-12-04 \mathrm{~T} 17: 42: 03$ \\
8 Spectrometer Frequency 100.61 \\
9 Spectral Width & 24038.5 \\
\hline
\end{tabular}

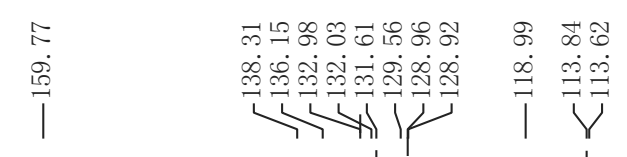

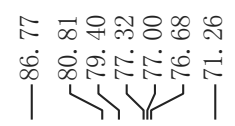

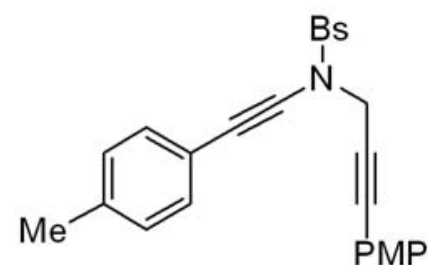

$1 \mathrm{i}$

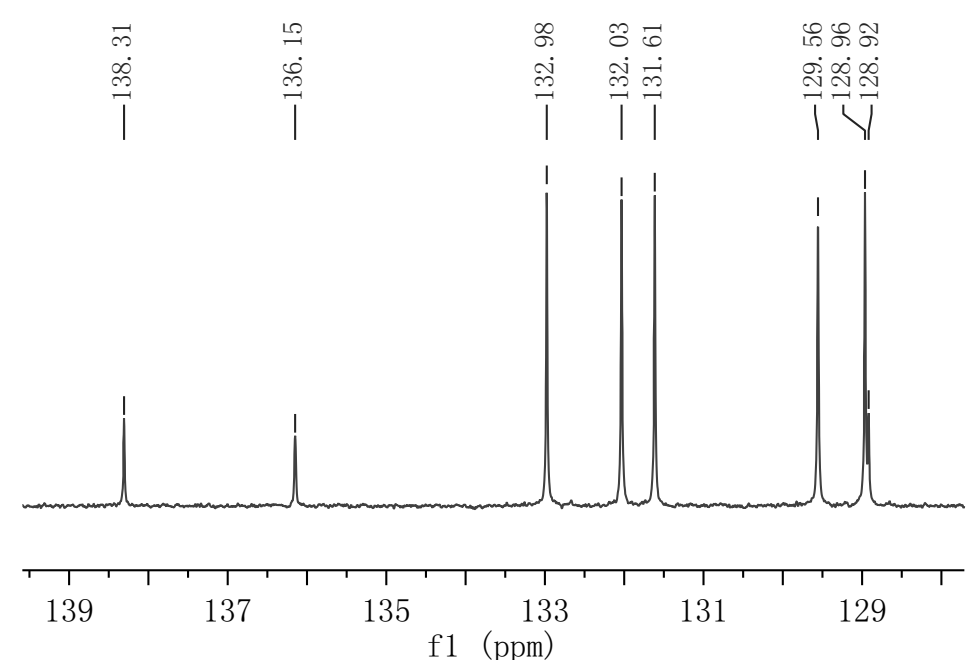

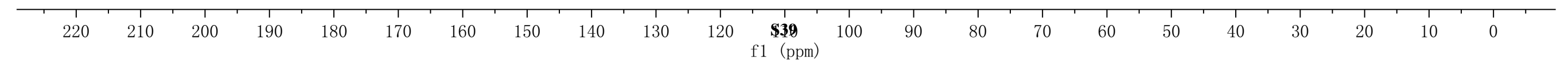




\begin{tabular}{|ll|}
\hline \multicolumn{1}{|c|}{ Parameter } & \multicolumn{1}{c|}{ Value } \\
1 Title & zgy-4-107-H \\
2 Origin & Bruker Bio\$pin GmbH \\
3 Solvent & CDC13 \\
4 Temperature & 298.0 \\
5 Number of Scans & 16 \\
6 Acquisition Tjme & 4.0894 \\
7 Acquisition Date & 2019-11-29T09:55:20 \\
8 Spectrometer Frequency & 400.13 \\
9 Spectral Width & 8012.8 \\
\hline
\end{tabular}
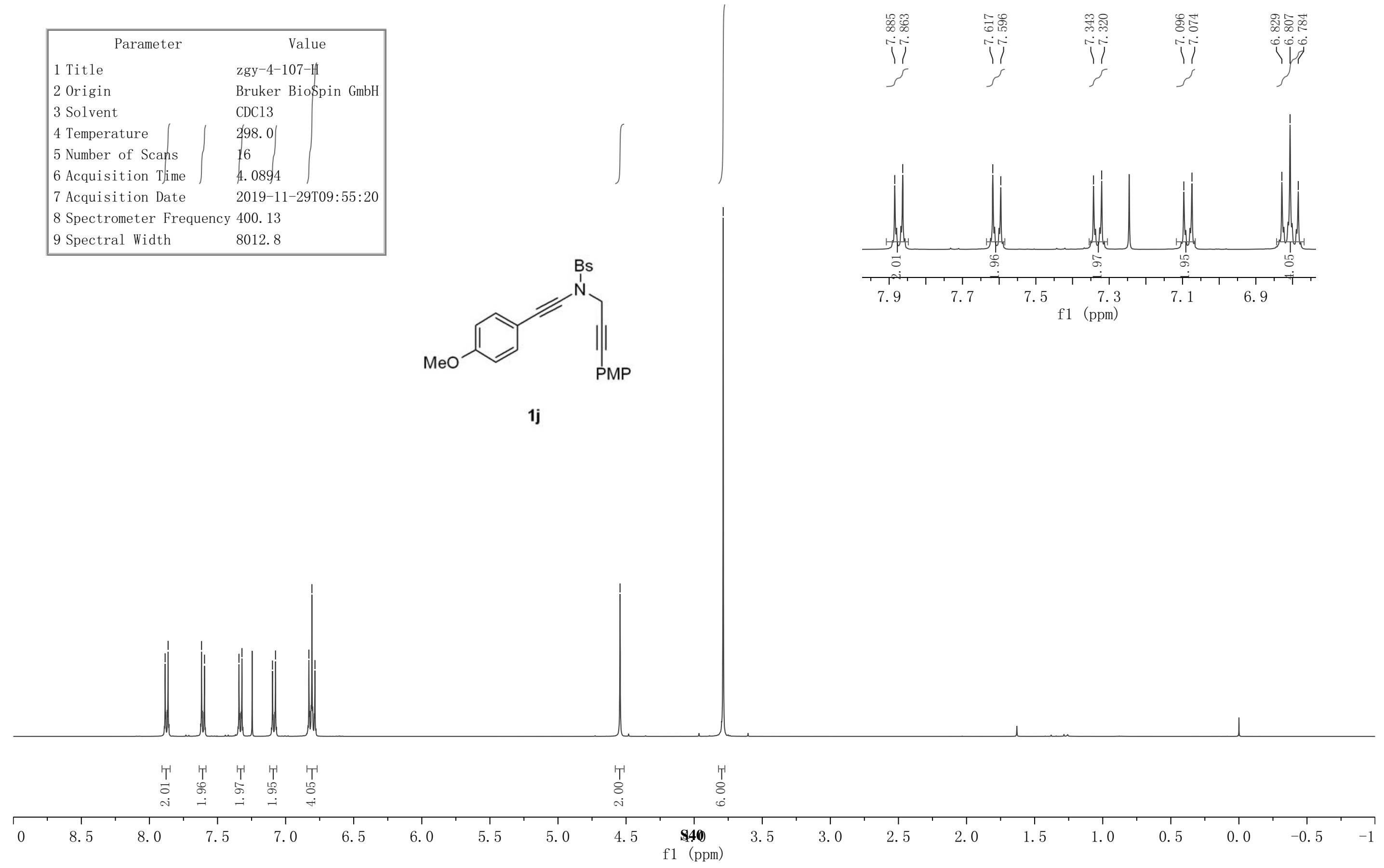


\begin{tabular}{|ll|}
\hline \multicolumn{1}{|c|}{ Parameter } & \multicolumn{1}{c|}{ Value } \\
1 Title & zgy-4-107-C \\
2 Origin & Bruker BioSpin GmbH \\
3 Solvent & CDC13 \\
4 Temperature & 300.0 \\
5 Number of Scans & 55 \\
6 Acquisition Time & 1.3631 \\
7 Acquisition Date & $2019-11-29 T 10: 00: 05$ \\
8 Spectrometer Frequency & 100.61 \\
9 Spectral Width & 24038.5 \\
\hline
\end{tabular}

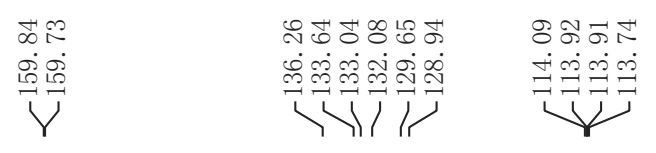

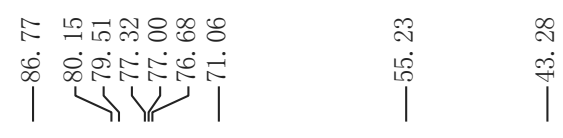

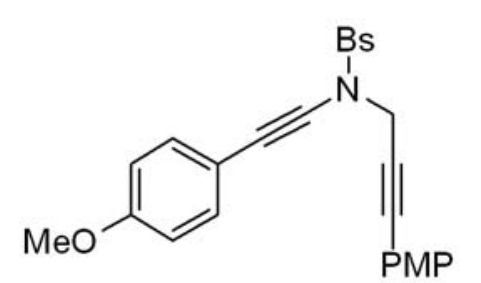

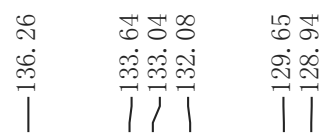

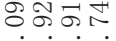

许

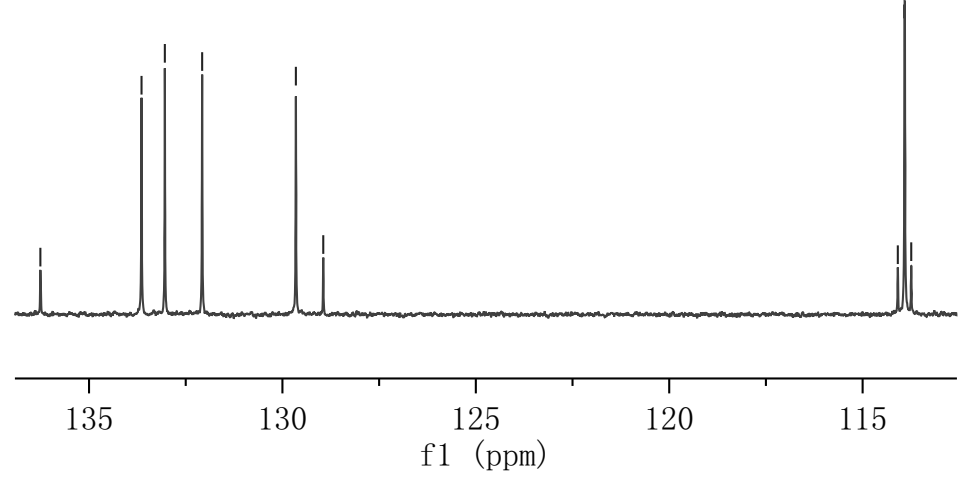

1j
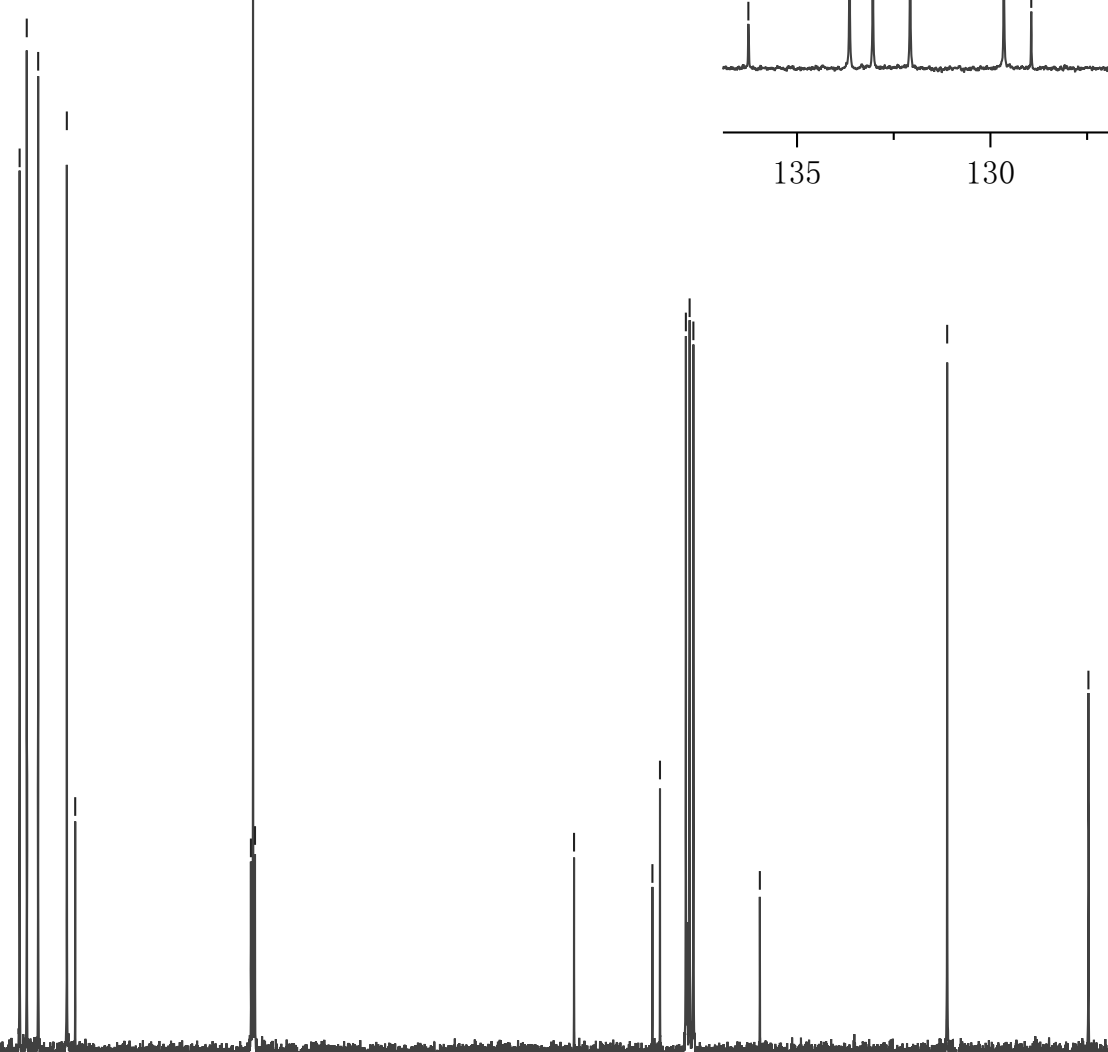

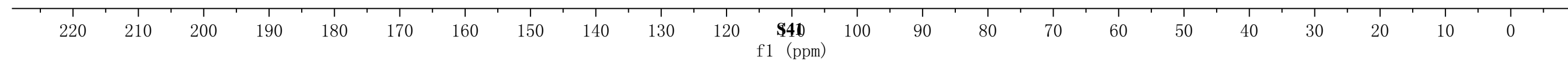




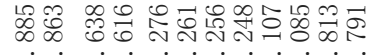

ओं

\begin{tabular}{|c|c|}
\hline Parameter & Value \\
\hline 1 Title & $\mathrm{zgy}-4-137-\mathrm{H}$ \\
\hline 2 Origin & Bruker BioSpin GmbH \\
\hline 3 Solvent & $\mathrm{CDC} 13$ \\
\hline 4 Temperature & 298.0 \\
\hline 5 Number of Scans & $19 \mid$ \\
\hline 6 Acquisition Time & 4. 08994 \\
\hline 7 Acquisition Date & $2019-12-12$ T09: $14: 36$ \\
\hline 8 Spectrometer Frequ & 400.13 \\
\hline 9 Spectral Width & 8012.8 \\
\hline
\end{tabular}

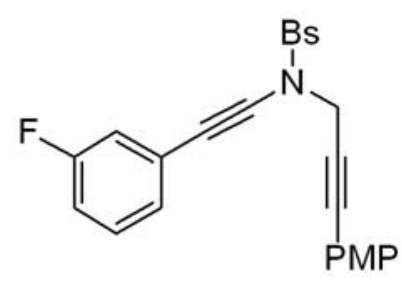

$1 k$

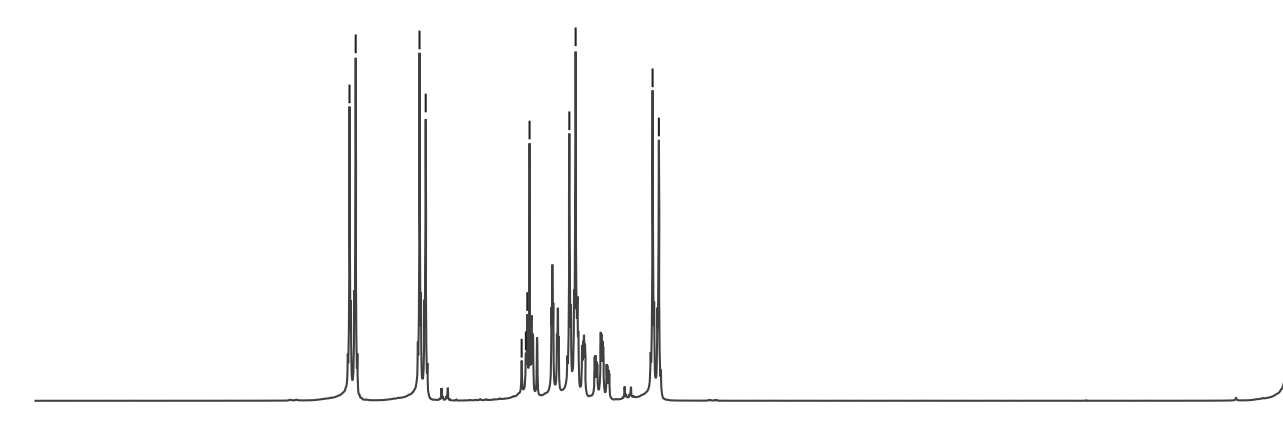

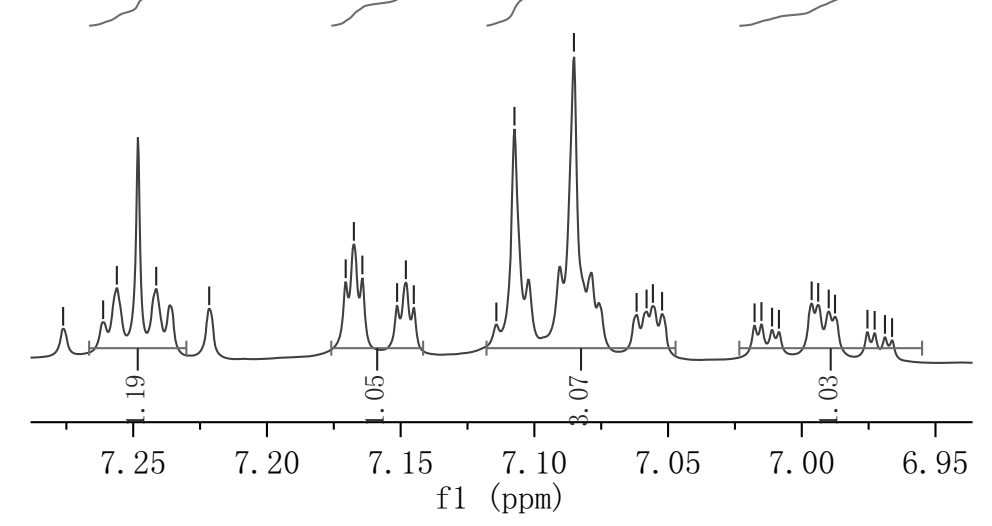

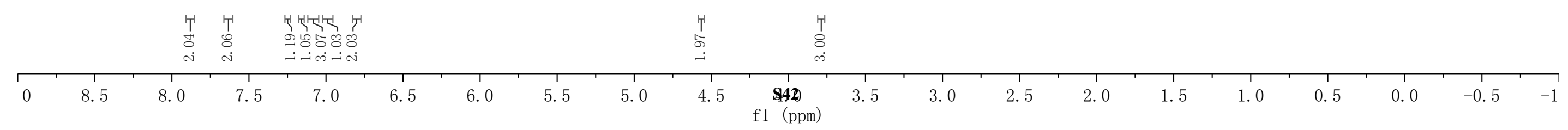




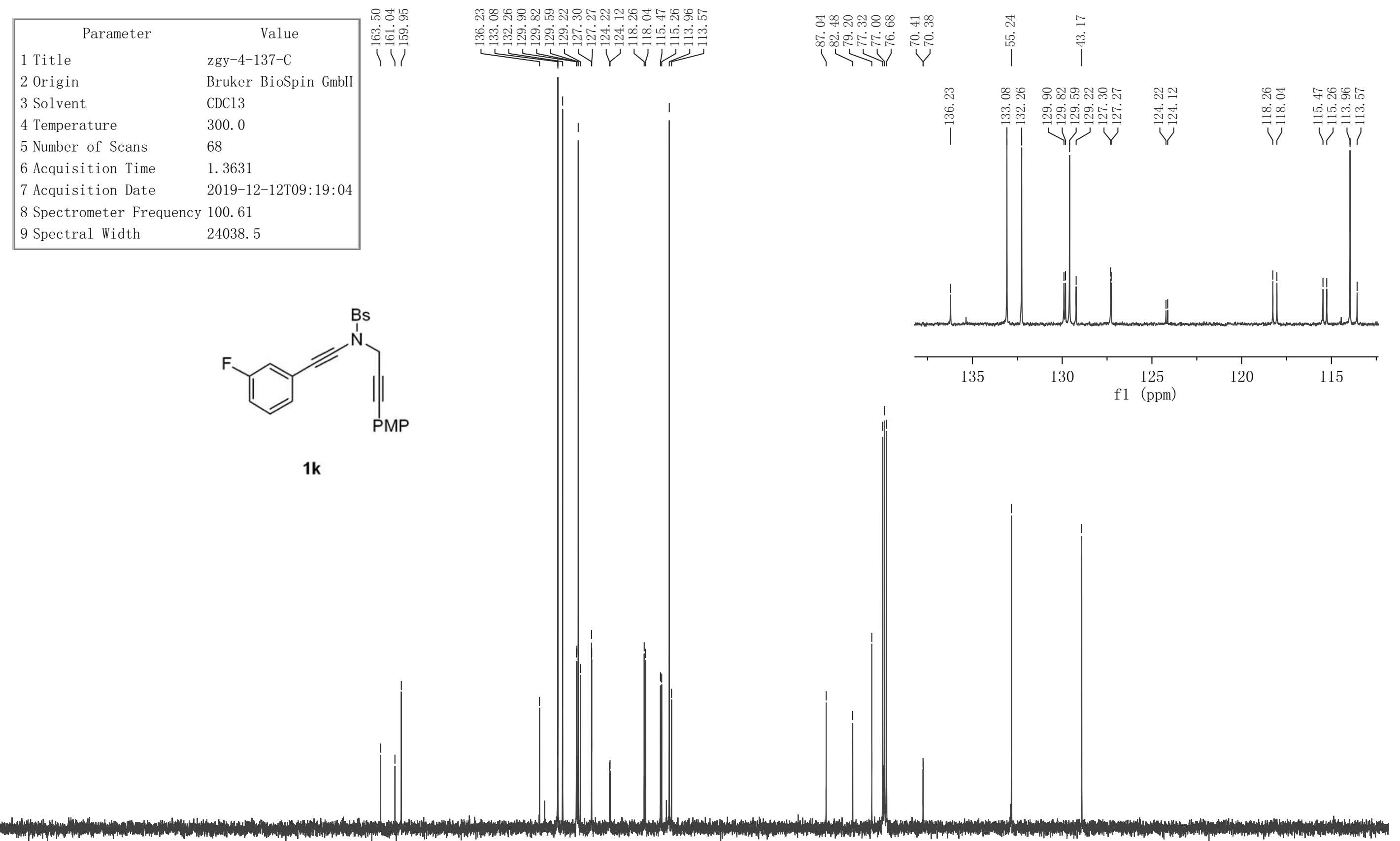




\begin{tabular}{|ll|}
\hline \multicolumn{1}{|c|}{ Parameter } & \multicolumn{1}{c|}{ Value } \\
1 Title & zgy-4-137-F \\
2 Origin & Bruker BioSpin GmbH \\
3 Solvent & CDC13 \\
4 Temperature & 297.1 \\
5 Number of Scans & 16 \\
6 Acquisition Time & 0.7340 \\
7 Acquisition Date & $2021-08-27$ T09:13:23 \\
8 Spectrometer Frequency & 376.31 \\
9 Spectral Width & 89285.7 \\
\hline
\end{tabular}

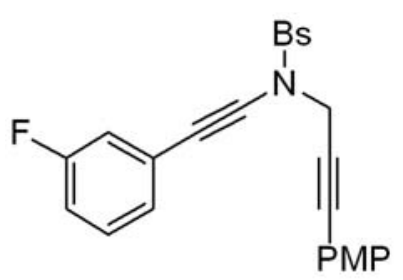

$1 k$

\begin{tabular}{|c|c|c|c|c|c|c|c|c|c|c|c|c|c|c|c|c|c|c|c|c|c|}
\hline 10 & 0 & -10 & -20 & -30 & -40 & -50 & -60 & -70 & -80 & -90 & $\begin{array}{c}\text { SHA0 } \\
\mathrm{f} 1 \stackrel{(\mathrm{ppm})}{ }\end{array}$ & -110 & -120 & -130 & -140 & -150 & -160 & -170 & -180 & -190 & $\begin{array}{ll}-200 & -210\end{array}$ \\
\hline
\end{tabular}




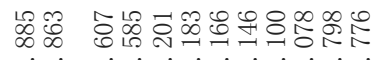

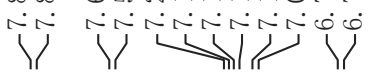

\begin{tabular}{|c|c|}
\hline \multicolumn{2}{|l|}{ Parameter } \\
\hline 1 Title & zgy-4-1 $28-\mathrm{H}$ \\
\hline 2 Origin & Bruker BioSpin $\mathrm{GmbH}$ \\
\hline 3 Solvent & $\mathrm{CDC} 13$ \\
\hline 4 Temperature & 298.0 \\
\hline 5 Number of Scans & 19 \\
\hline 6 Acquisition Time & 4. 0894 \\
\hline 7 Acquisition Date & 2019-12-10T09:06:44 \\
\hline 8 Spectrometer Fue & 400.13 \\
\hline 9 Spectral Width & 8012.8 \\
\hline
\end{tabular}

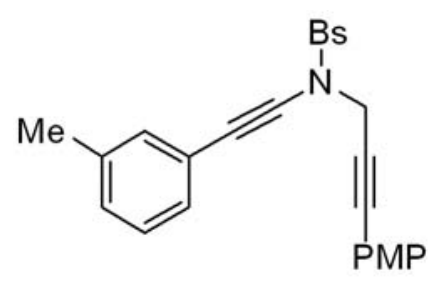

11
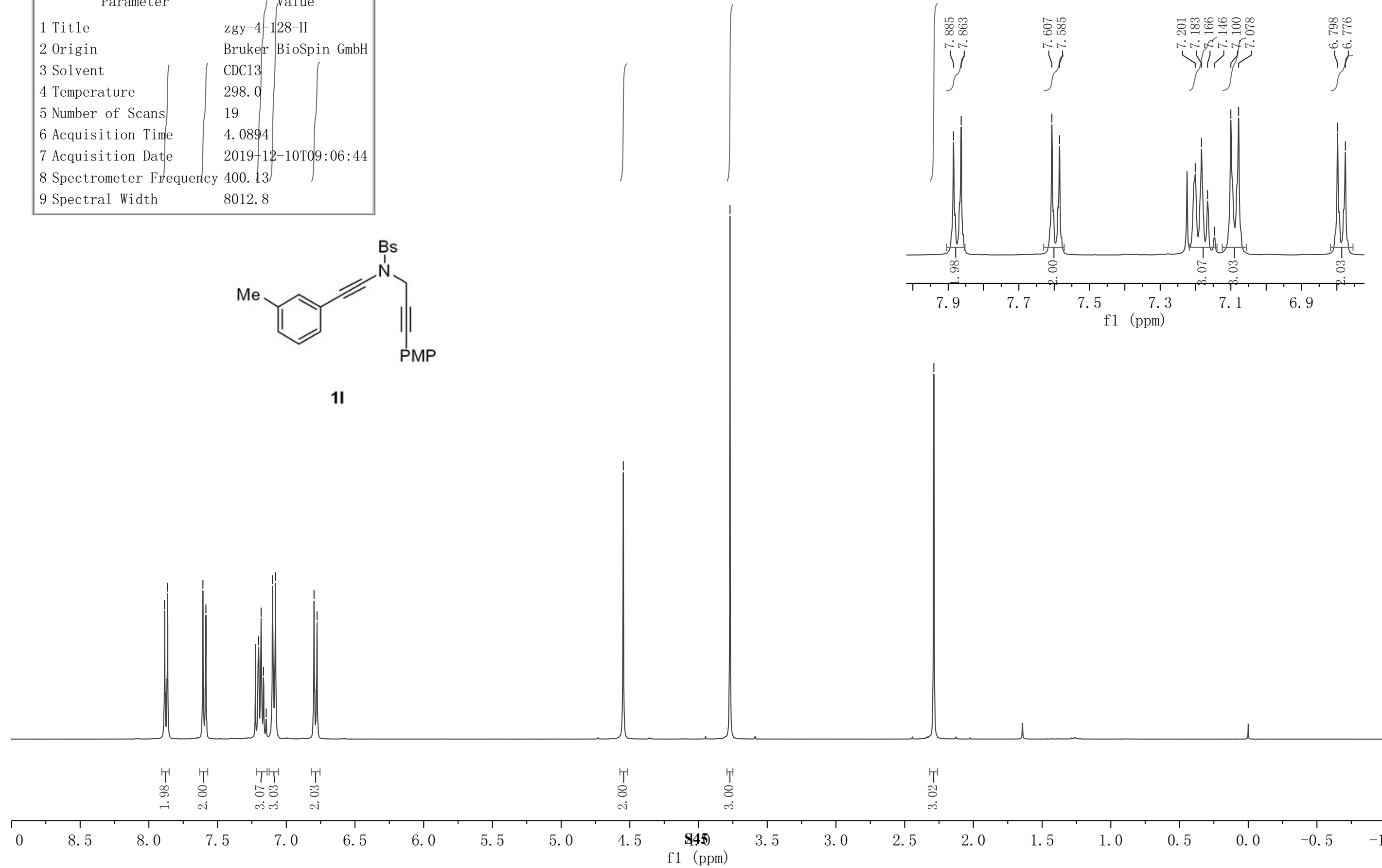


\begin{tabular}{|ll|}
\hline \multicolumn{1}{|c|}{ Parameter } & \multicolumn{1}{c|}{ Value } \\
1 Title & zgy-4-128-C \\
2 Origin & Bruker BioSpin GmbH \\
3 Solvent & CDC13 \\
4 Temperature & 300.0 \\
5 Number of Scans & 33 \\
6 Acquisition Time & 1.3631 \\
7 Acquisition Date & $2019-12-10 T 09: 11: 39$ \\
8 Spectrometer Frequency 100.61 \\
9 Spectral Width & 24038.5 \\
\hline
\end{tabular}

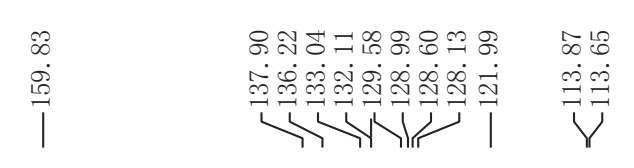

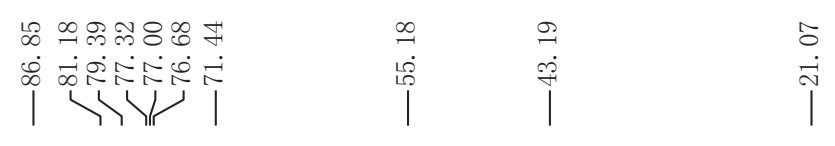

re

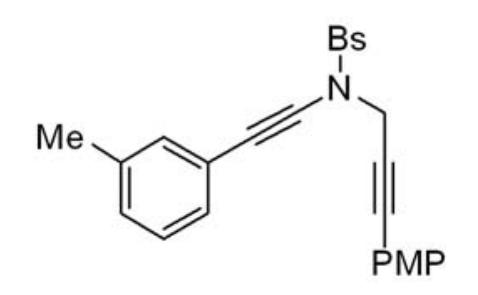

11
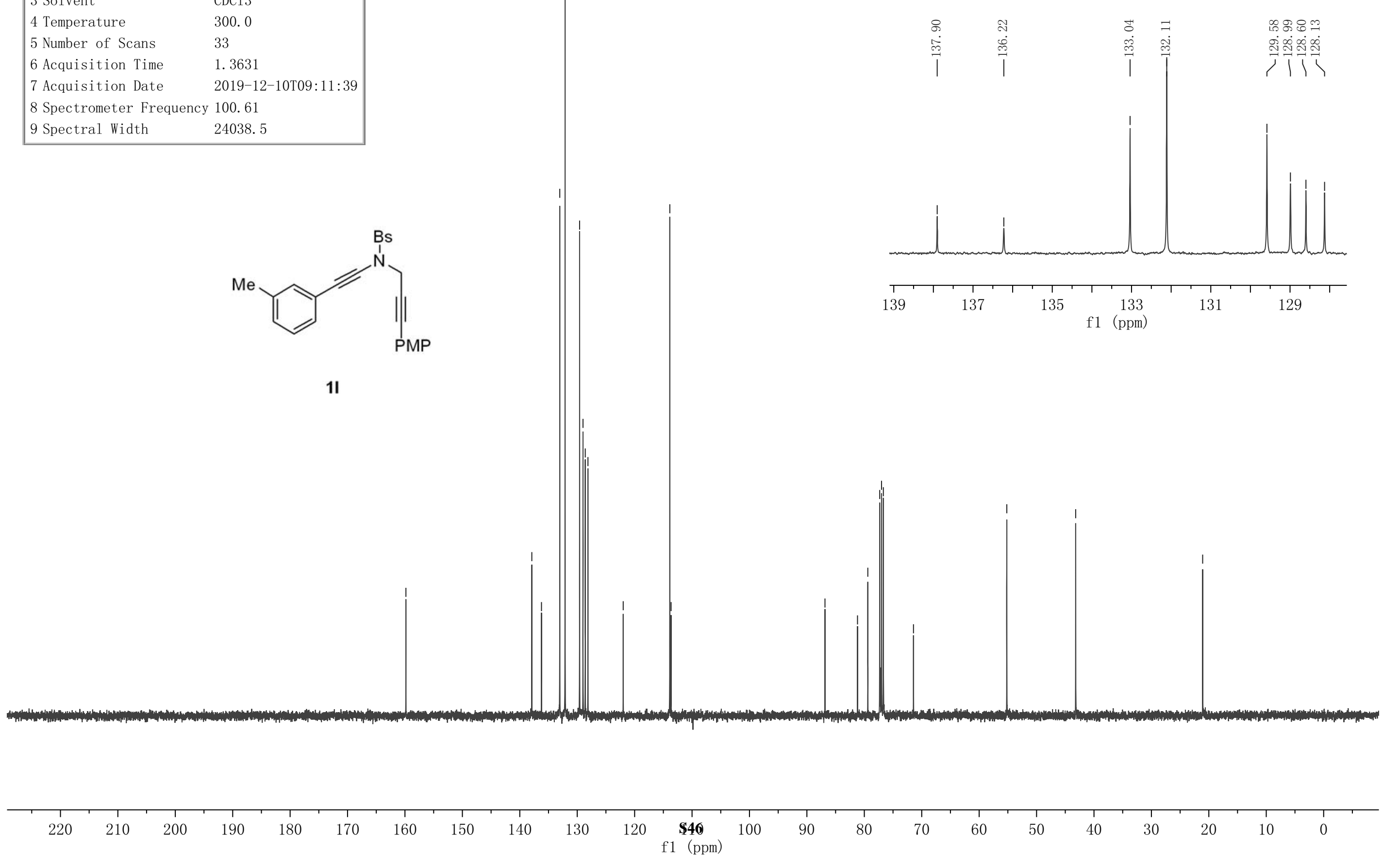


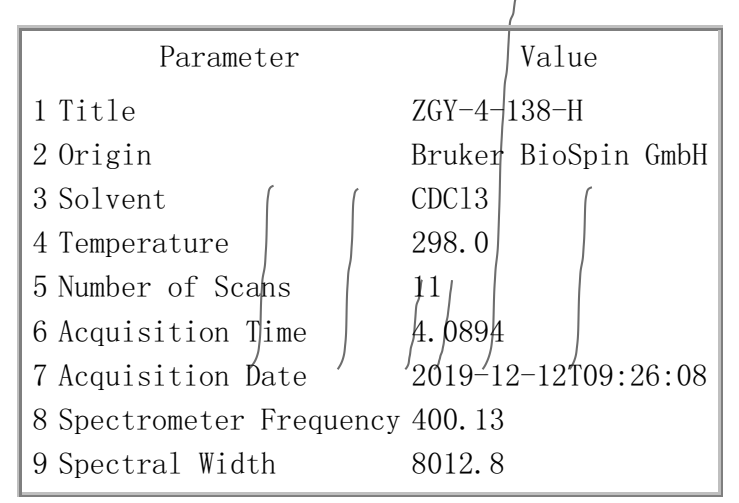
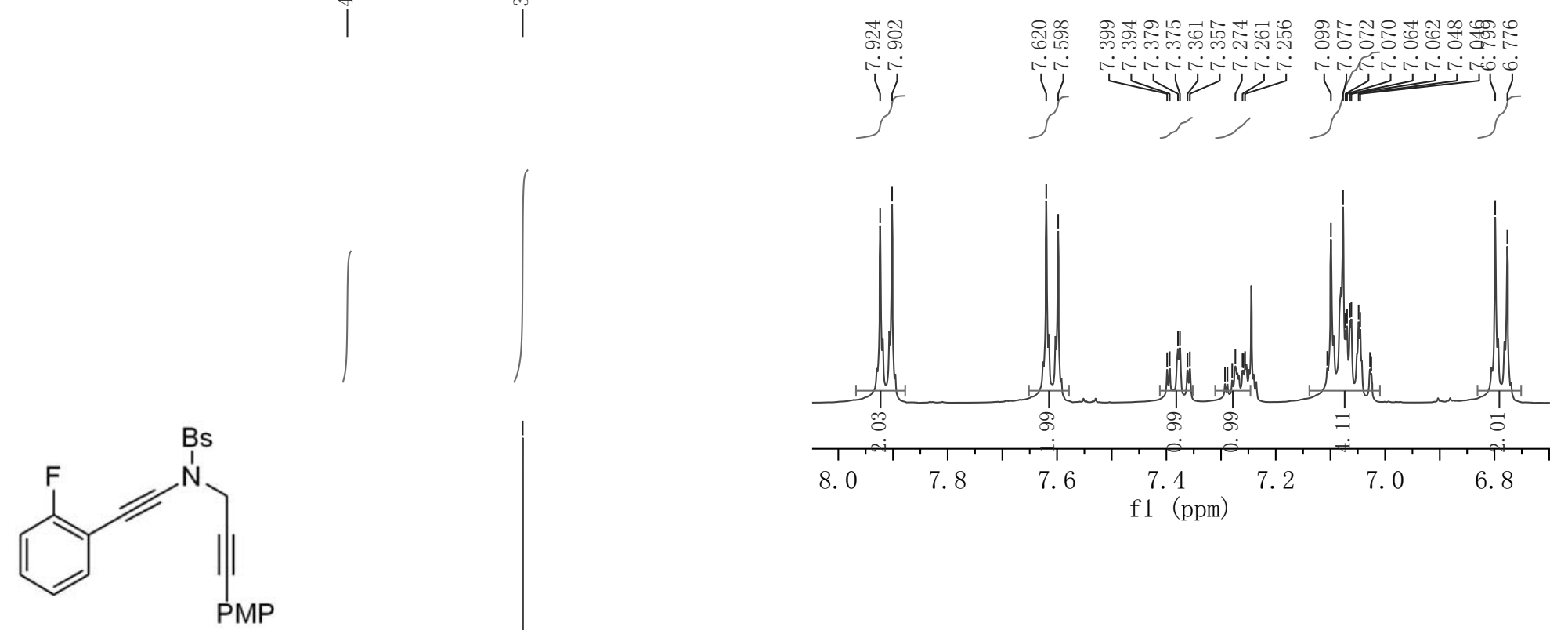

$1 \mathrm{~m}$

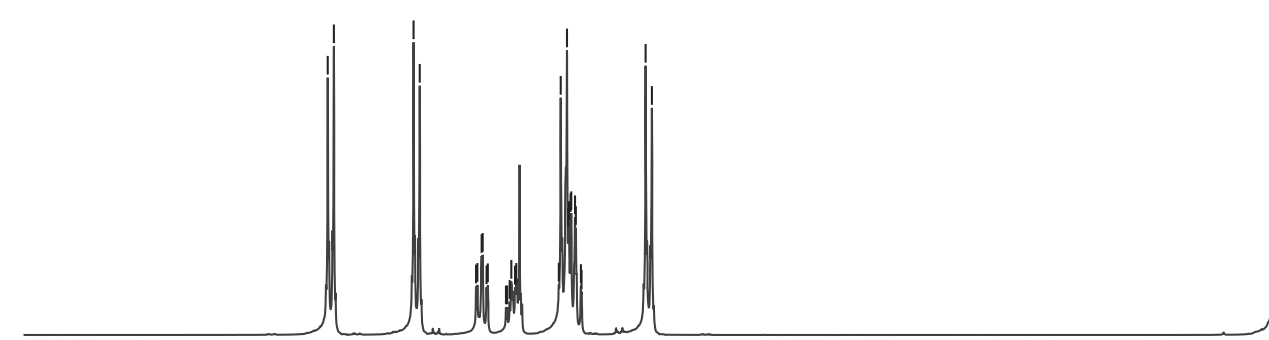

\begin{tabular}{|c|c|c|c|c|c|c|c|c|c|c|c|c|c|c|c|c|c|c|c|c|}
\hline & & $\begin{array}{l}T^{1} \\
8 \\
\delta \\
i \\
\dot{0}\end{array}$ & $\begin{array}{l}T^{\top} \\
\text { S } \\
\stackrel{-}{-}\end{array}$ & 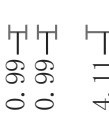 & 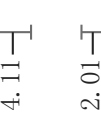 & & & & & $\begin{array}{l}T^{\top} \\
\infty \\
\infty \\
-\end{array}$ & $\begin{array}{l}\top \\
8 \\
8 \\
\dot{\infty}\end{array}$ & & & & & & & & & \\
\hline 0 & 8.5 & 8.0 & 7.5 & & 7. 0 & 6.5 & 6.0 & 5.5 & 5. 0 & 4.5 & $\begin{array}{c}\mathbf{8 4 7} \\
\mathrm{f} 1 \stackrel{(\mathrm{ppm})}{ }\end{array}$ & 3.5 & 3. 0 & 2.5 & 2.0 & 1.5 & 1.0 & 0.5 & 0.0 & -0.5 \\
\hline
\end{tabular}




\begin{tabular}{|ll|}
\hline \multicolumn{1}{|c|}{ Parameter } & \multicolumn{1}{c}{ Value } \\
1 Title & ZGY-4-138-C \\
2 Origin & Bruker BioSpin GmbH \\
3 Solvent & CDCl3 \\
4 Temperature & 300.0 \\
5 Number of Scans & 82 \\
6 Acquisition Time & 1.3631 \\
7 Acquisition Date & $2019-12-12 \mathrm{~T} 09: 29: 31$ \\
8 Spectrometer Frequency & 100.61 \\
9 Spectral Width & 24038.5 \\
\hline
\end{tabular}

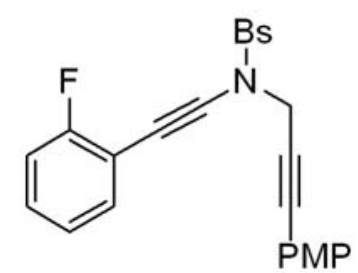

$1 \mathrm{~m}$

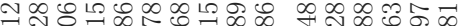

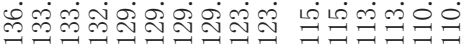
난

๑ำ

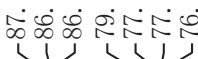

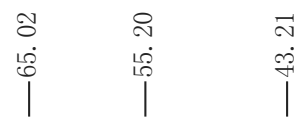

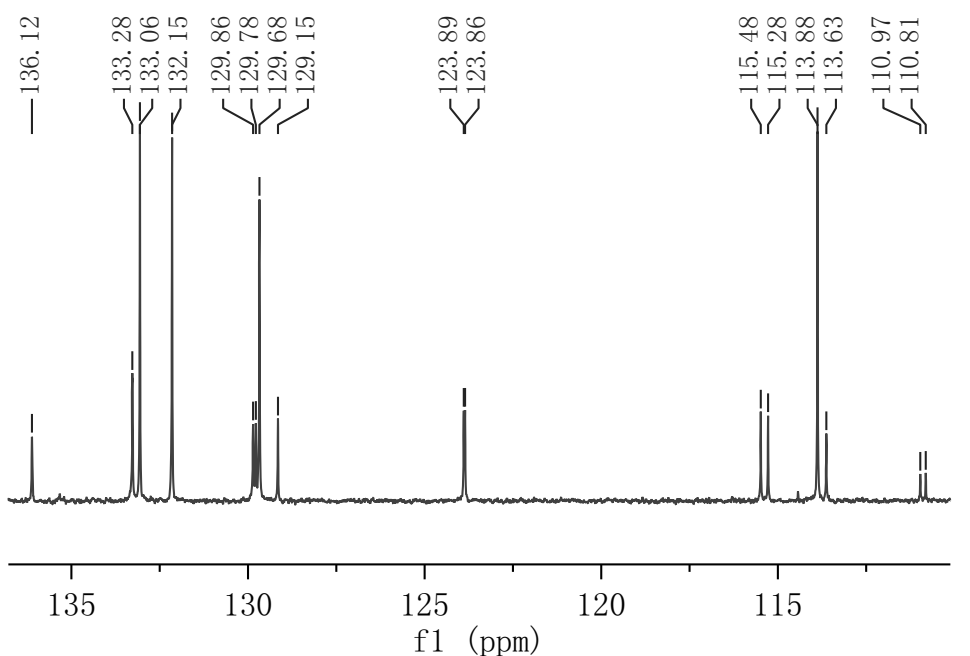




\begin{tabular}{|ll|}
\hline \multicolumn{1}{|c|}{ Parameter } & \multicolumn{1}{c|}{ Value } \\
1 Title & ZGY-4-138-F \\
2 Origin & Bruker BioSpin GmbH \\
3 Solvent & CDC13 \\
4 Temperature & 297.4 \\
5 Number of Scans & 16 \\
6 Acquisition Time & 0.7340 \\
7 Acquisition Date & $2021-08-27$ T18:07:59 \\
8 Spectrometer Frequency 376.31 \\
9 Spectral Width & 89285.7 \\
\hline
\end{tabular}

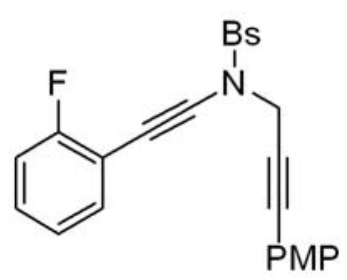

$1 \mathrm{~m}$

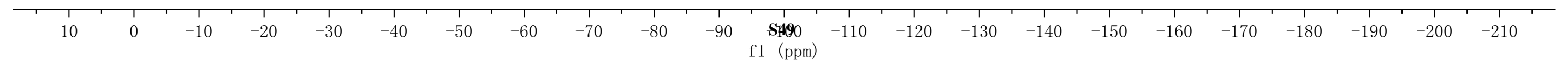




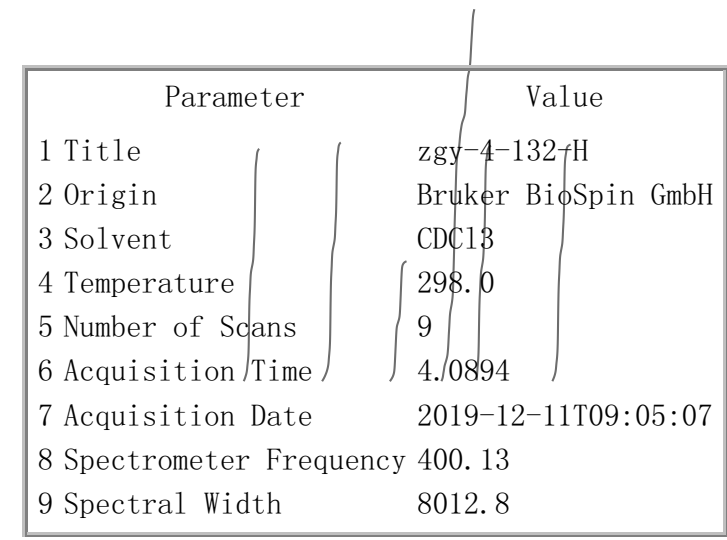
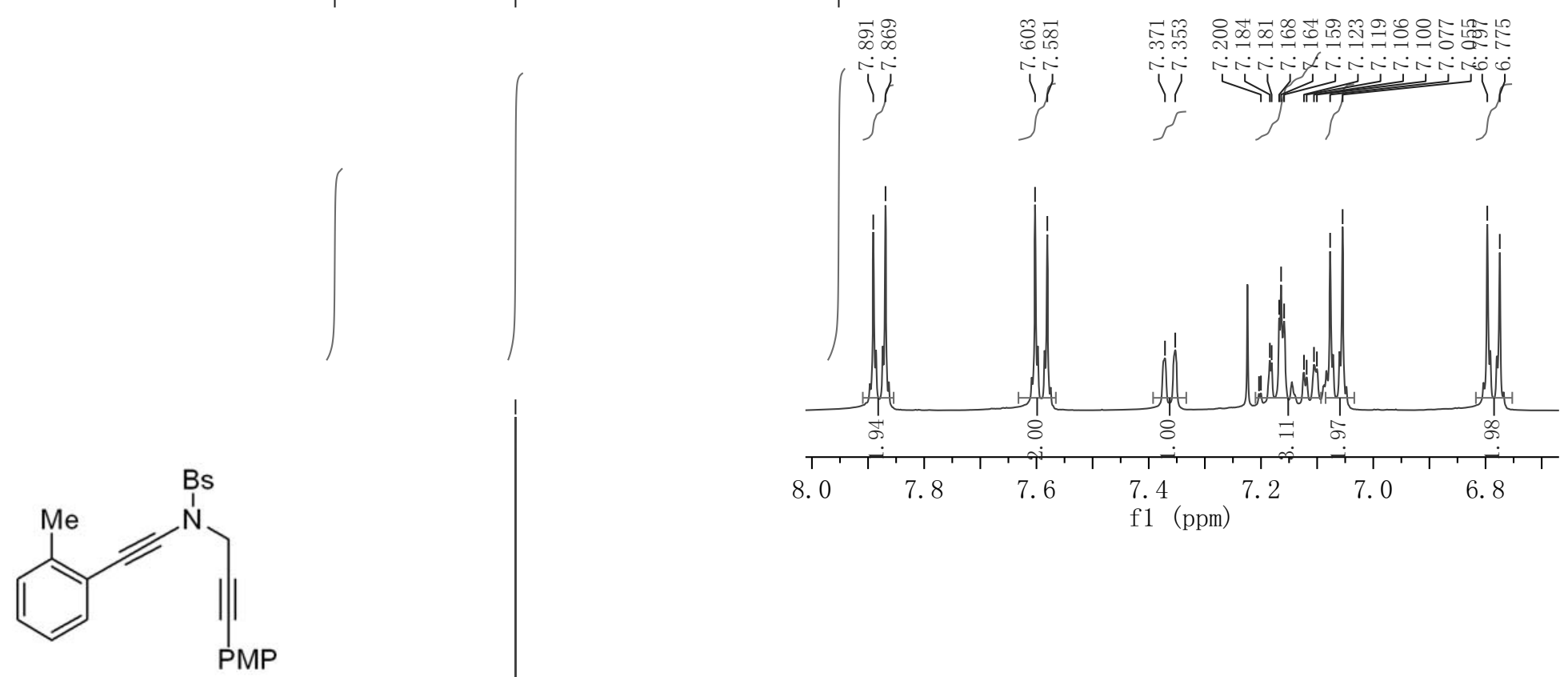

$1 n$

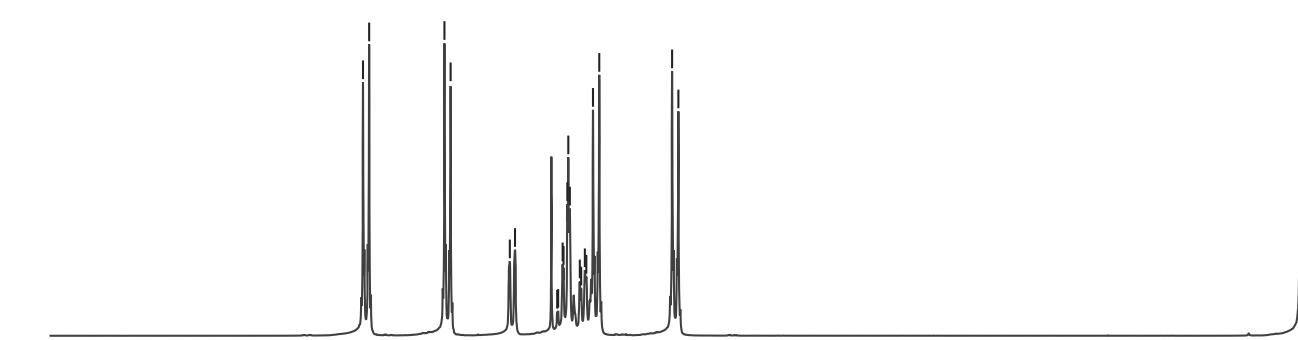

\begin{tabular}{|c|c|c|c|c|c|c|c|c|c|c|c|c|c|c|c|c|c|c|c|}
\hline & & $\begin{array}{l}T \\
\text { Tे } \\
-\end{array}$ & $\begin{array}{ll}T & T \\
8 & 8 \\
8 & \stackrel{1}{\prime} \\
i & -\end{array}$ & 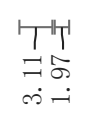 & $\begin{array}{l}T \\
\infty \\
\infty \\
- \\
-1\end{array}$ & & & & $\begin{array}{l}\text { T' } \\
8 \\
\text { i } \\
\text { in }\end{array}$ & $\begin{array}{l}\text { 'T } \\
8 \\
\dot{8}\end{array}$ & & & $\begin{array}{l}\text { T' } \\
\qquad 8 \\
\dot{8} \\
\dot{\infty}\end{array}$ & & & & & & \\
\hline 0 & 8.5 & 8.0 & 7.5 & 7. 0 & 6.5 & 6.0 & 5.5 & 5. 0 & 4. 5 & $\begin{array}{c}\mathbf{9 5 0} \\
\mathrm{f} 1 \stackrel{(\mathrm{ppm})}{ }\end{array}$ & 3.5 & 3. 0 & 2.5 & 2.0 & 1.5 & 1. 0 & 0.5 & 0.0 & -0.5 \\
\hline
\end{tabular}




\begin{tabular}{|ll|}
\hline \multicolumn{1}{|c|}{ Parameter } & \multicolumn{1}{c|}{ Value } \\
1 Title & zgy-4-132-C \\
2 Origin & Bruker BioSpin GmbH \\
3 Solvent & CDC13 \\
4 Temperature & 300.0 \\
5 Number of Scans & 48 \\
6 Acquisition Time & 1.3631 \\
7 Acquisition Date & $2019-12-11 \mathrm{~T} 09: 07: 53$ \\
8 Spectrometer Frequency & 100.61 \\
9 Spectral Width & 24038.5 \\
\hline
\end{tabular}
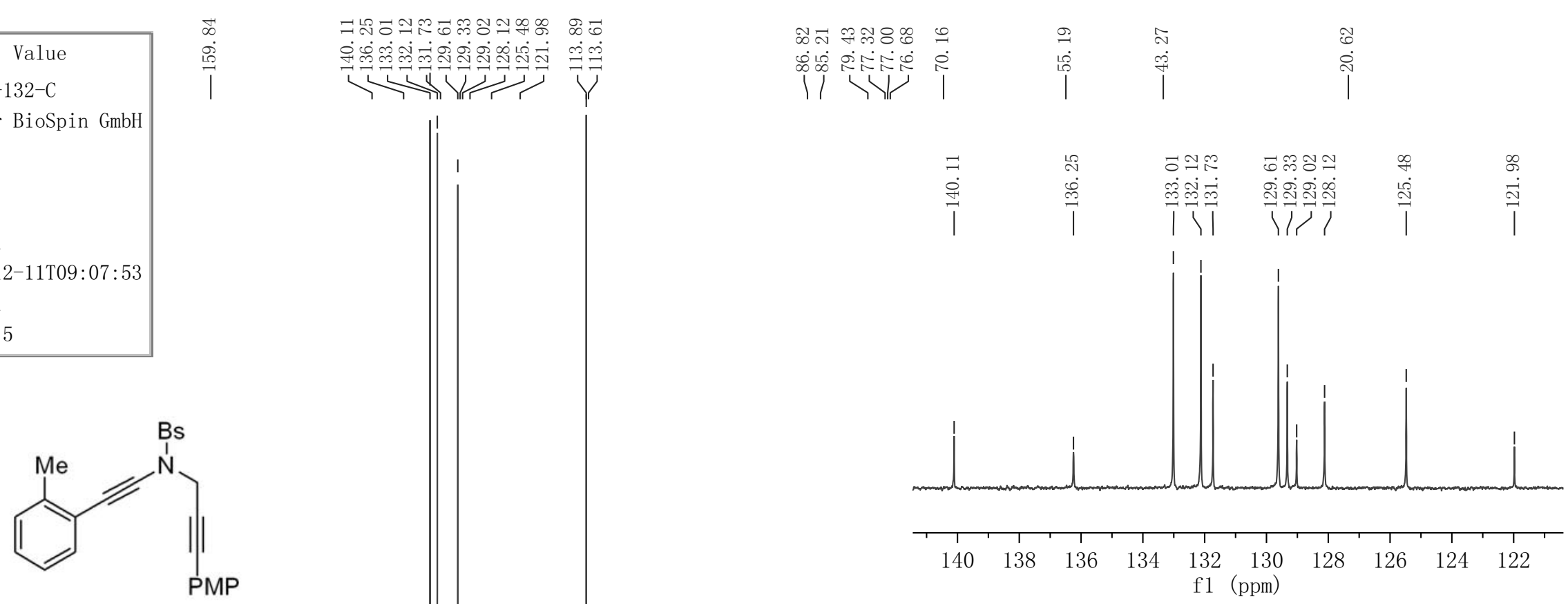

$1 n$

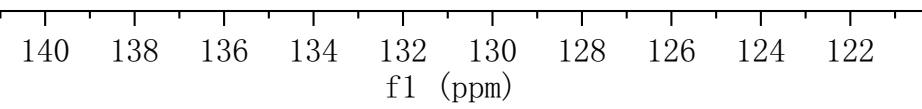

.

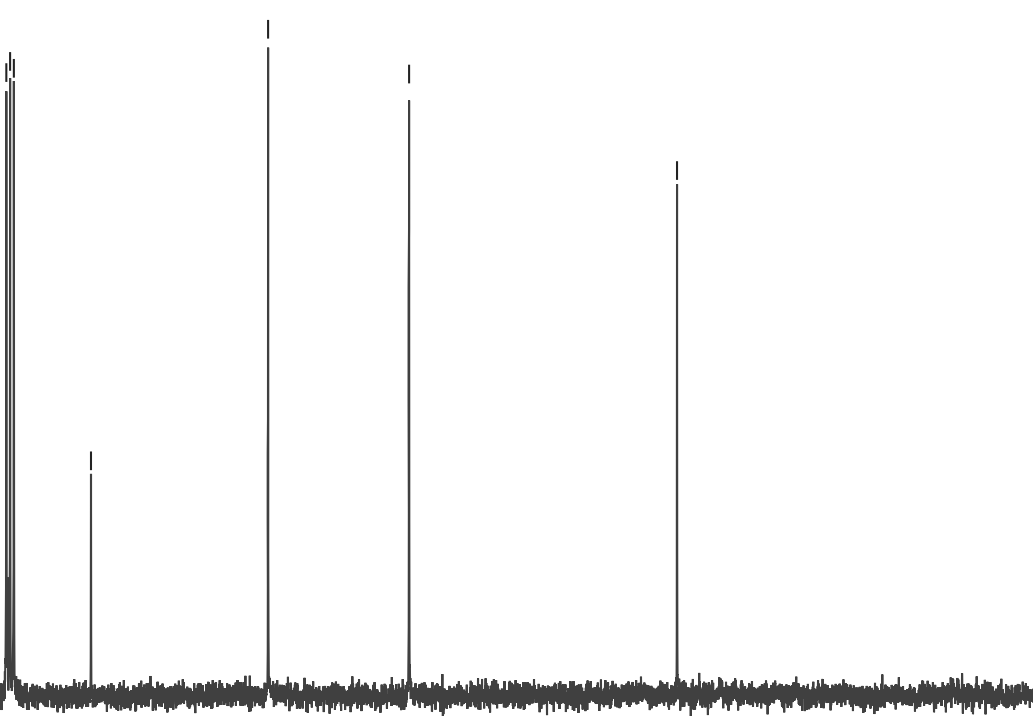


Parameter

\section{Value}

\section{Title}

2 Origin

3 Solvent

4 Temperature

5 Number of Scans

6 Acquisition Time

7 Acquisition Date $2019-12-14 \mathrm{P} 14: 12: 35$

8 Spectrometer Frequency 400. 13

9 Spectral Width

8012.8

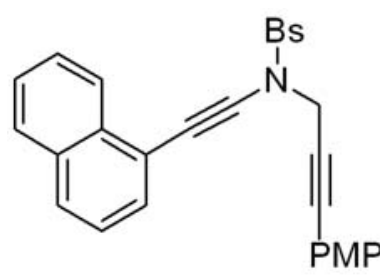

10
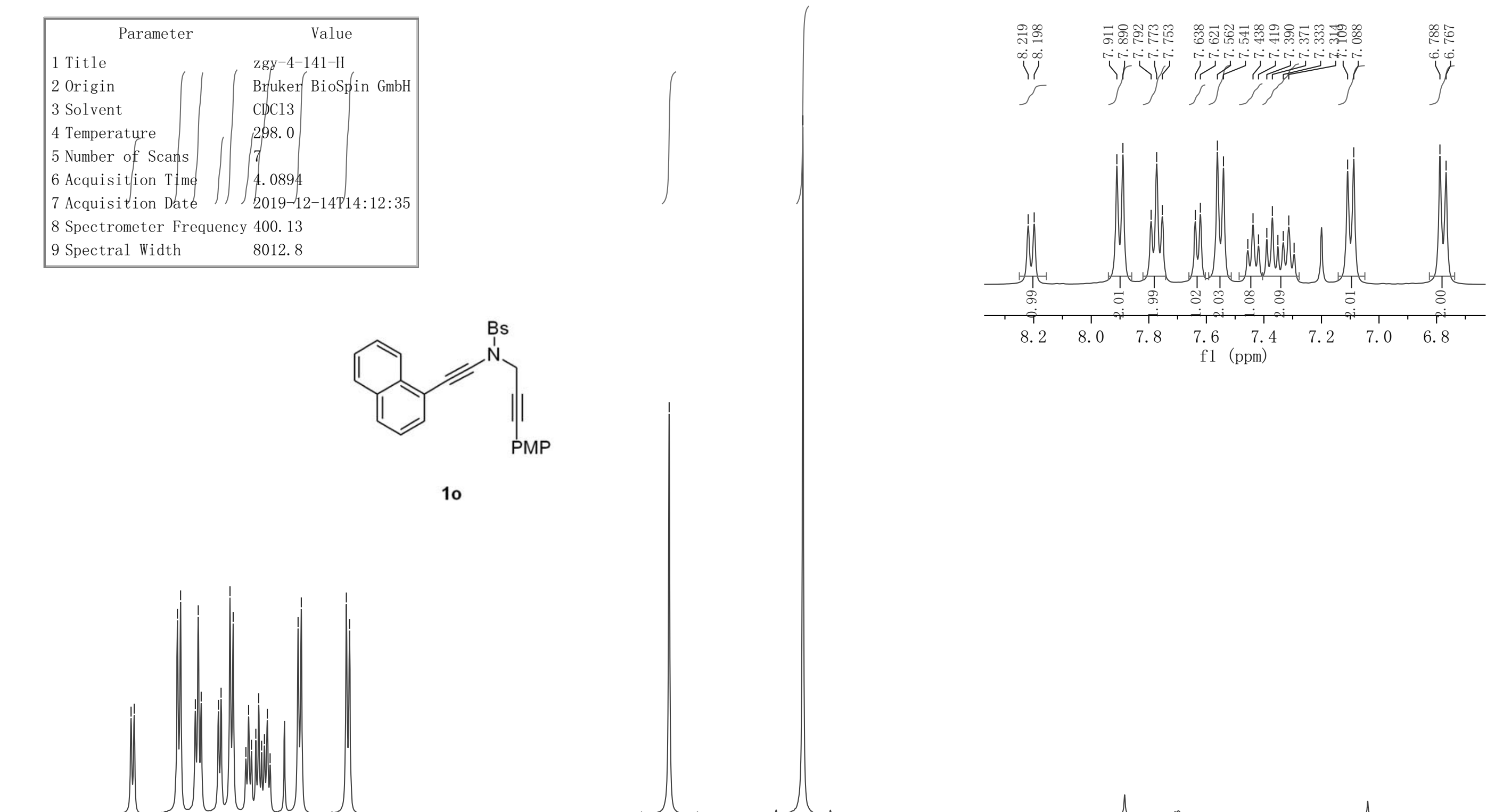

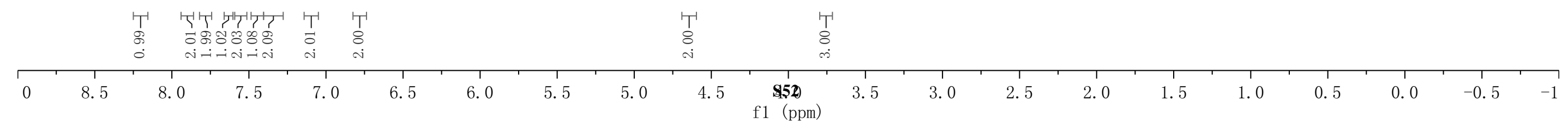




\begin{tabular}{|ll|}
\hline \multicolumn{1}{|c|}{ Parameter } & \multicolumn{1}{c|}{ Value } \\
1 Title & zgy-4-141-C \\
2 Origin & Bruker BioSpin GmbH \\
3 Solvent & CDC13 \\
4 Temperature & 300.0 \\
5 Number of Scans & 43 \\
6 Acquisition Time & 1.3631 \\
7 Acquisition Date & $2019-12-14 \mathrm{~T} 14: 15: 27$ \\
8 Spectrometer Frequency & 100.61 \\
9 Spectral Width & 24038.5 \\
\hline
\end{tabular}

9 Spectral
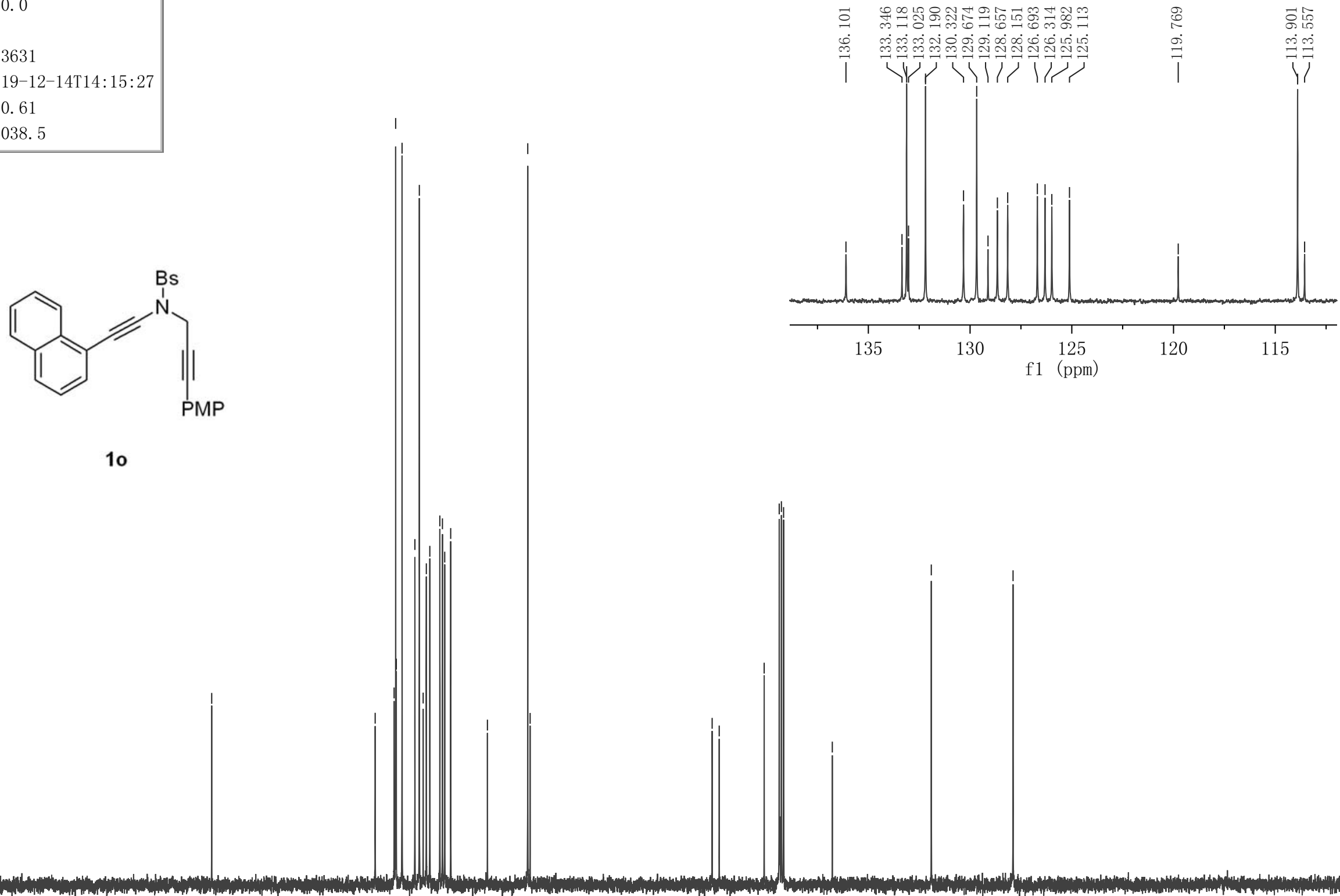

10

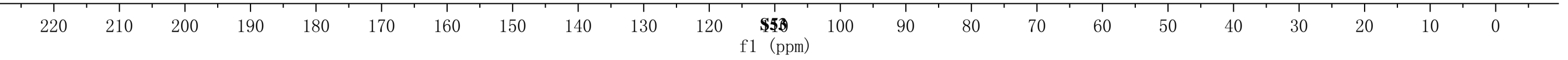




\begin{tabular}{|c|c|}
\hline Parameter & Value \\
\hline 1 Title & $\mathrm{zgy}-4-150-\mathrm{H}$ \\
\hline 2 Origin & Bruker Biospin $\mathrm{GmbH}$ \\
\hline 3 Solvent & $\mathrm{CDCl} 3$ \\
\hline 4 Temperature & 298.0 \\
\hline 5 Number of Scans & 6 \\
\hline 6 Acquisition Time & 4. 0894 \\
\hline 7 Acquisition Date & 2019-12-18T16:01:08 \\
\hline 8 Spectrometer Freq & 400.13 \\
\hline 9 Spectral Width & 8012.8 \\
\hline
\end{tabular}
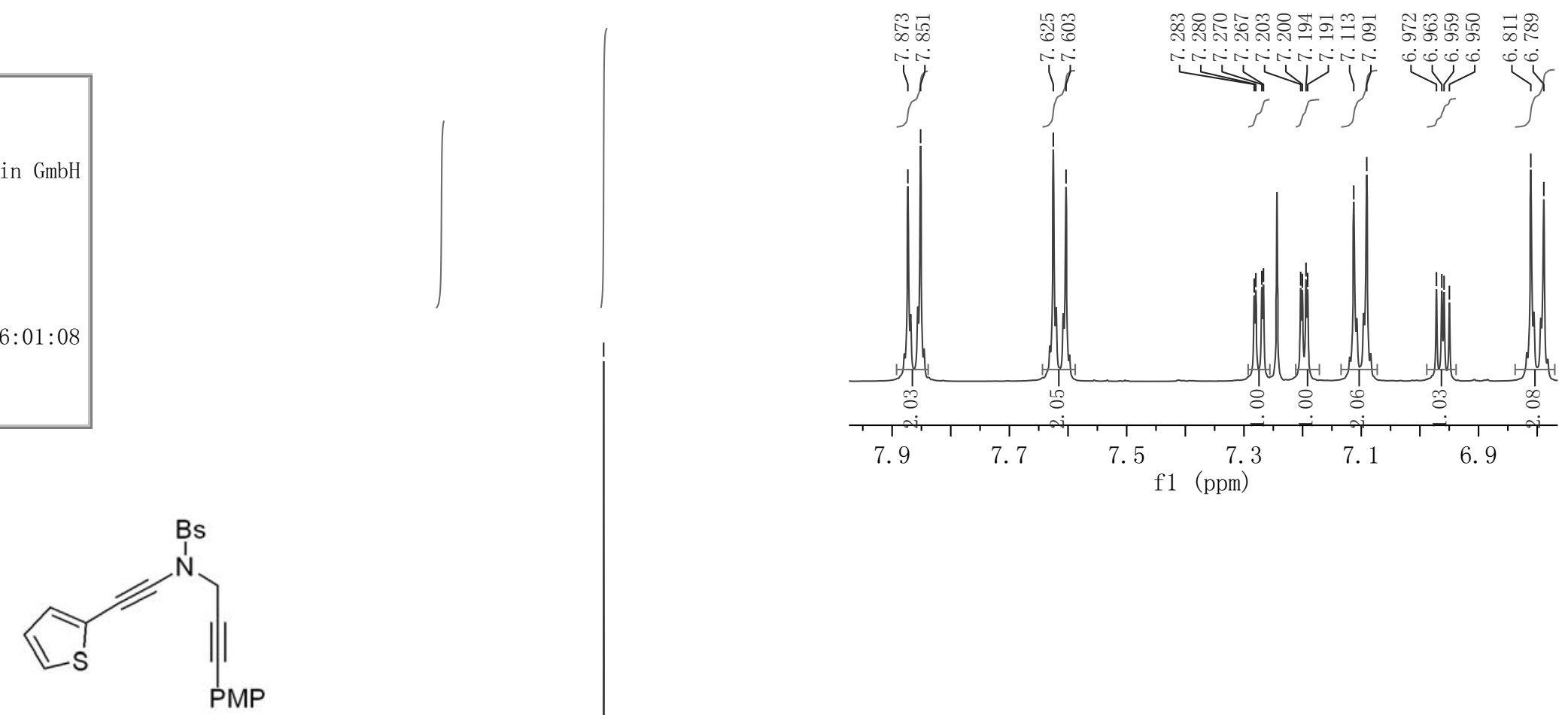

$1 p$

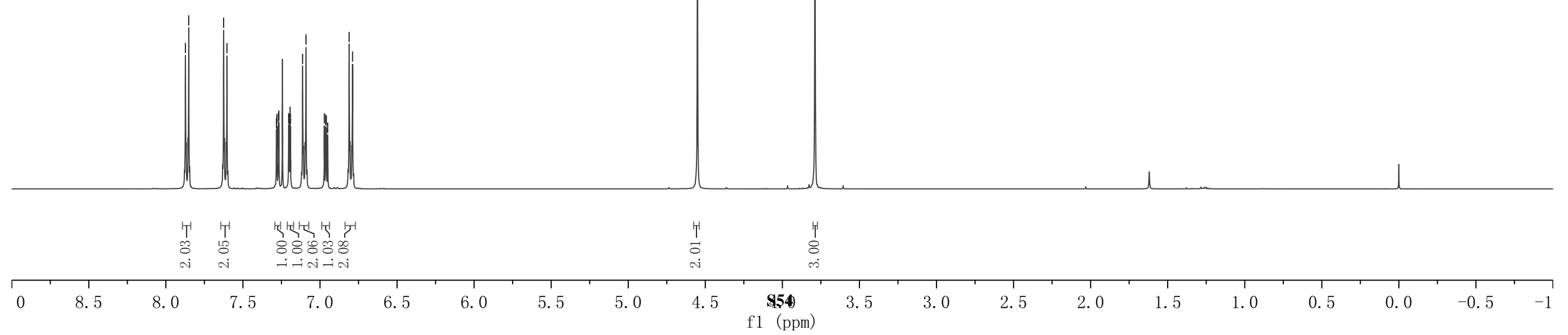




\begin{tabular}{|ll|}
\hline \multicolumn{1}{|c|}{ Parameter } & \multicolumn{1}{c|}{ Value } \\
1 Title & zgy-4-150-C \\
2 Origin & Bruker BioSpin GmbH \\
3 Solvent & CDC13 \\
4 Temperature & 300.0 \\
5 Number of Scans & 30 \\
6 Acquisition Time & 1.3631 \\
7 Acquisition Date & $2019-12-18 \mathrm{~T} 16: 02: 20$ \\
8 Spectrometer Frequency 100.61 \\
9 Spectral Width & 24038.5 \\
\hline
\end{tabular}

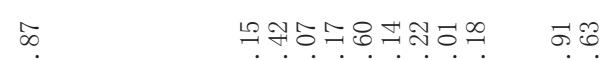

|
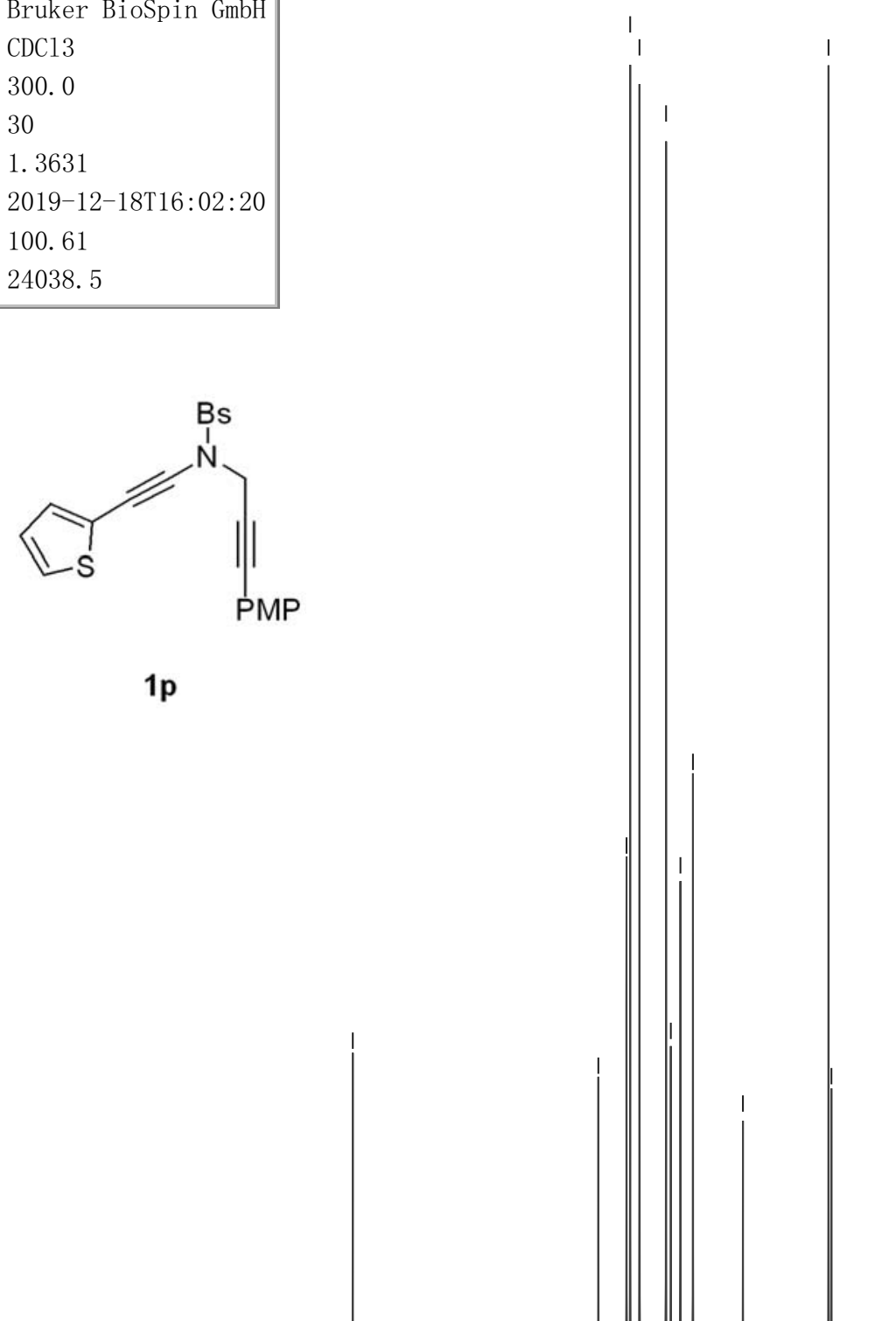

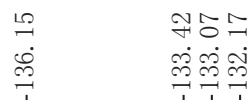

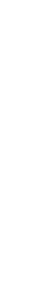

$1 p$

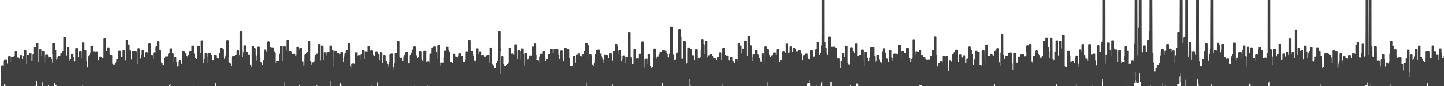

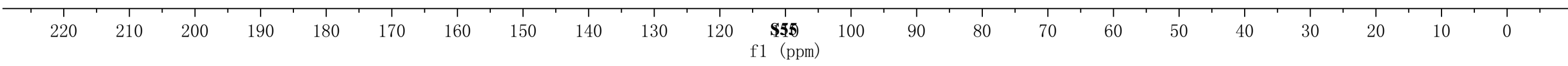




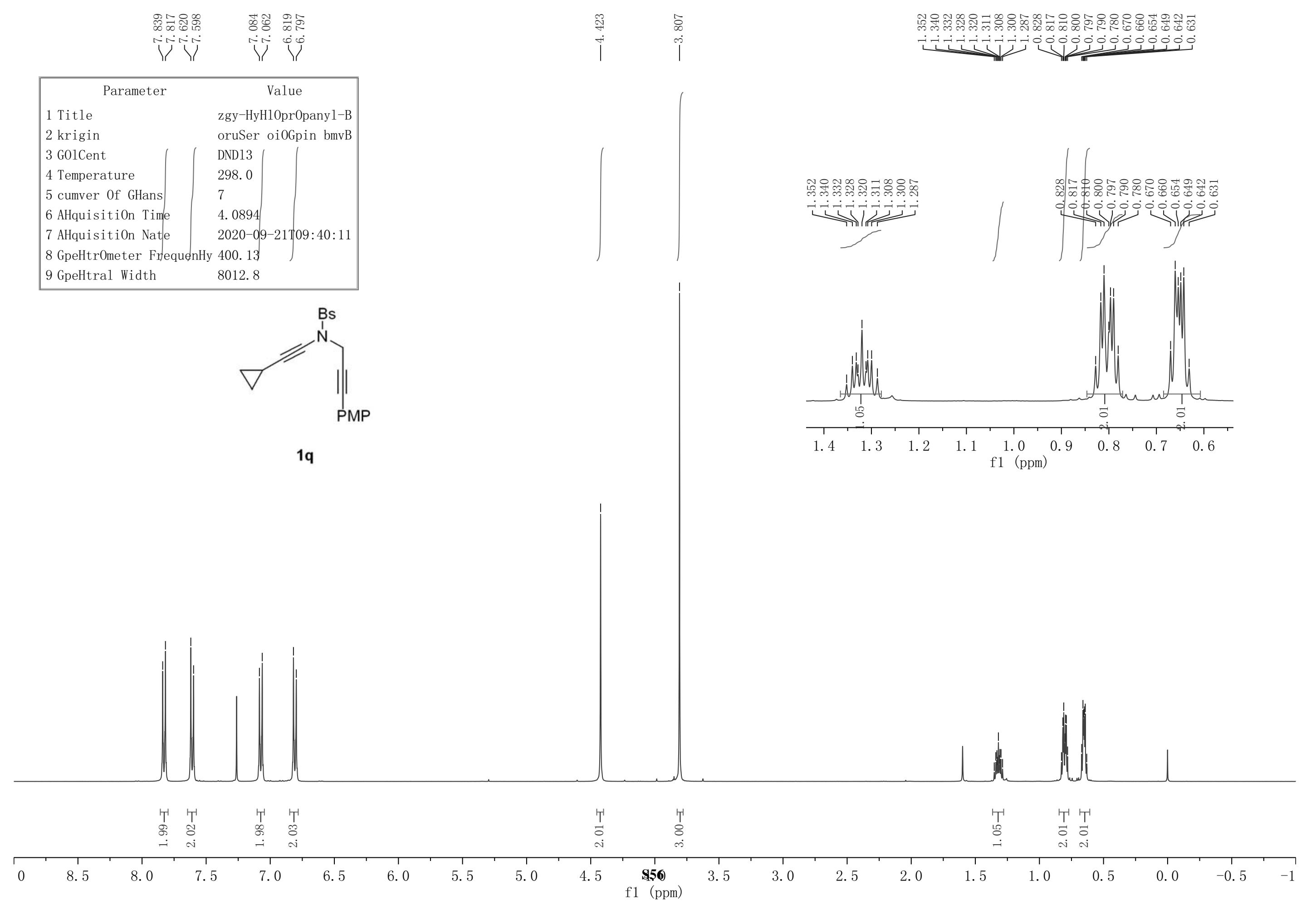




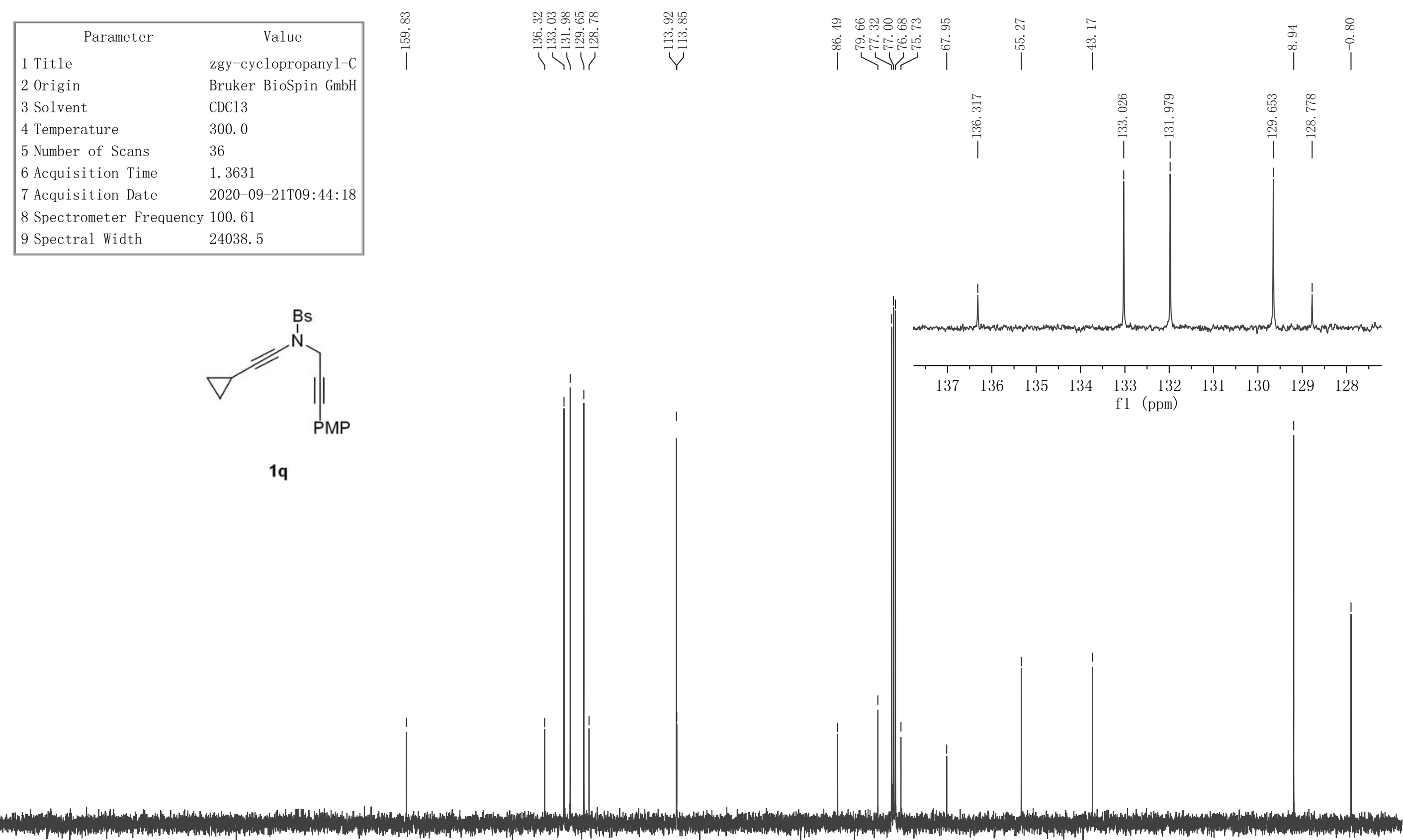




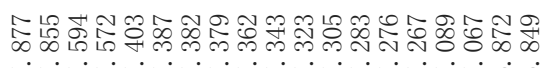

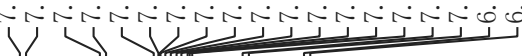

\begin{tabular}{|c|c|}
\hline Parameter & Value \\
\hline 1 Title & $z g y-5-196-H$ \\
\hline 2 Origin & Bruker BioSpin GmbH \\
\hline 3 Solvent & dDC13 \\
\hline 4 Temperature & 299.4 \\
\hline 5 Number of Scans & 14 \\
\hline 6 Acquisition Time & 3. 9846 \\
\hline 7 Acquisition Date & $2020-09-16 \mathrm{~T} 10: 05: 52$ \\
\hline \multicolumn{2}{|c|}{8 Spectrometer Frequency 400. 03} \\
\hline 9 Spectral Width & 8223.7 \\
\hline
\end{tabular}

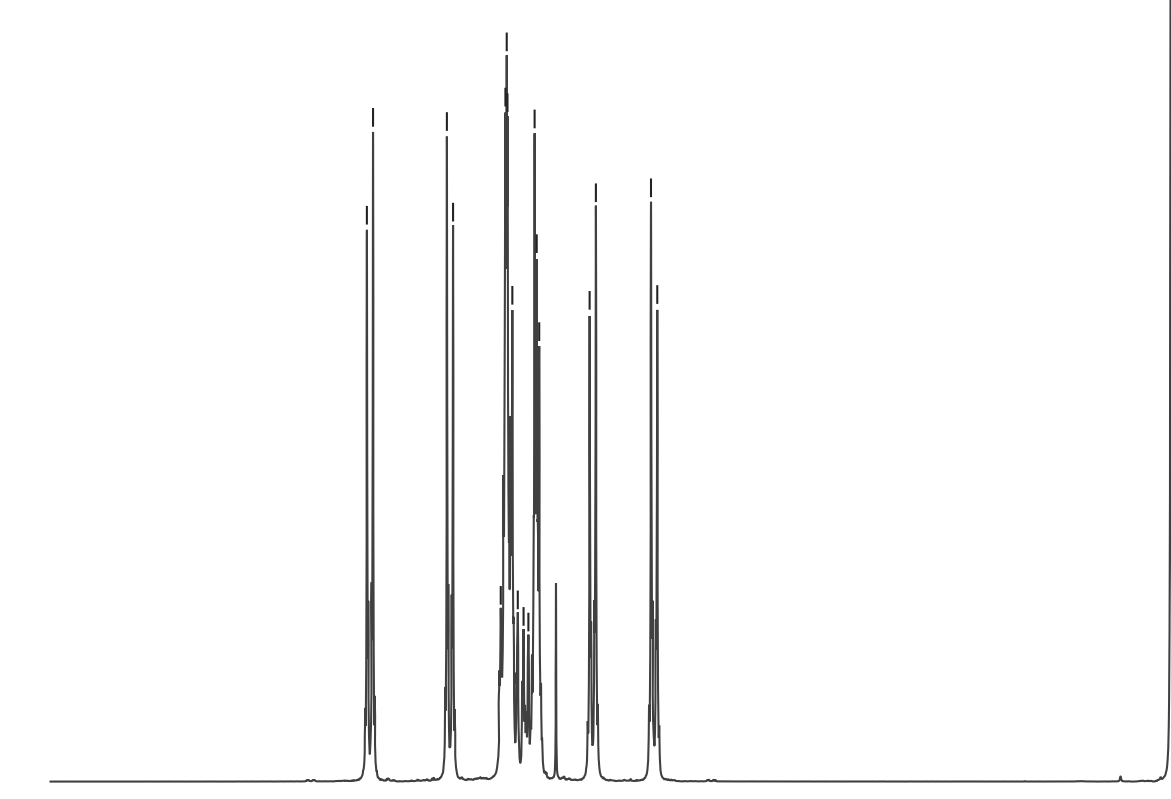

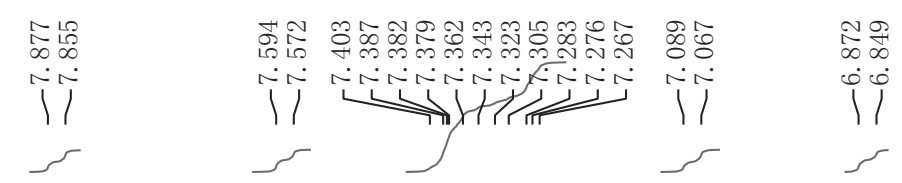

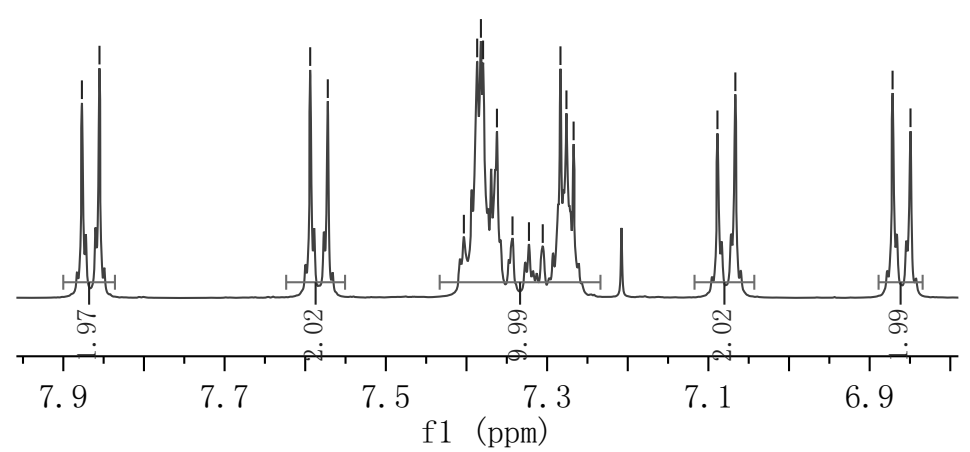

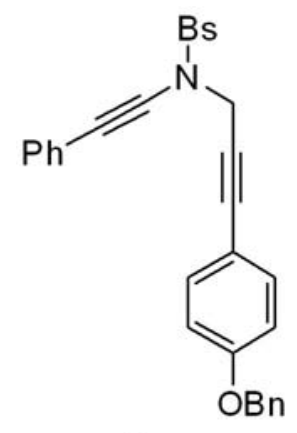

$1 \mathrm{r}$

\begin{tabular}{|c|c|c|c|c|c|c|c|c|c|c|c|c|c|c|c|c|c|c|c|c|}
\hline & & \begin{tabular}{l}
$\top$ \\
\multirow{\sigma}{*}{} \\
- \\
-1
\end{tabular} & $\begin{array}{l}\text { T' } \\
\text { ò } \\
i\end{array}$ & 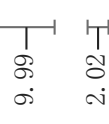 & 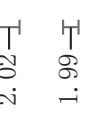 & & & & 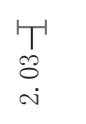 & $\begin{array}{l}\text { 'T' } \\
8 \\
\dot{0} \\
\dot{i}\end{array}$ & & & & & & & & & & \\
\hline 0 & 8.5 & 8.0 & 7.5 & & 7. 0 & 6.5 & 6.0 & 5.5 & 5. 0 & 4.5 & $\begin{array}{c}\mathbf{9 5 5} \\
\mathrm{f1}(\mathrm{ppm})\end{array}$ & 3.5 & 3. 0 & 2.5 & 2.0 & 1.5 & 1.0 & 0.5 & 0.0 & -0.5 \\
\hline
\end{tabular}



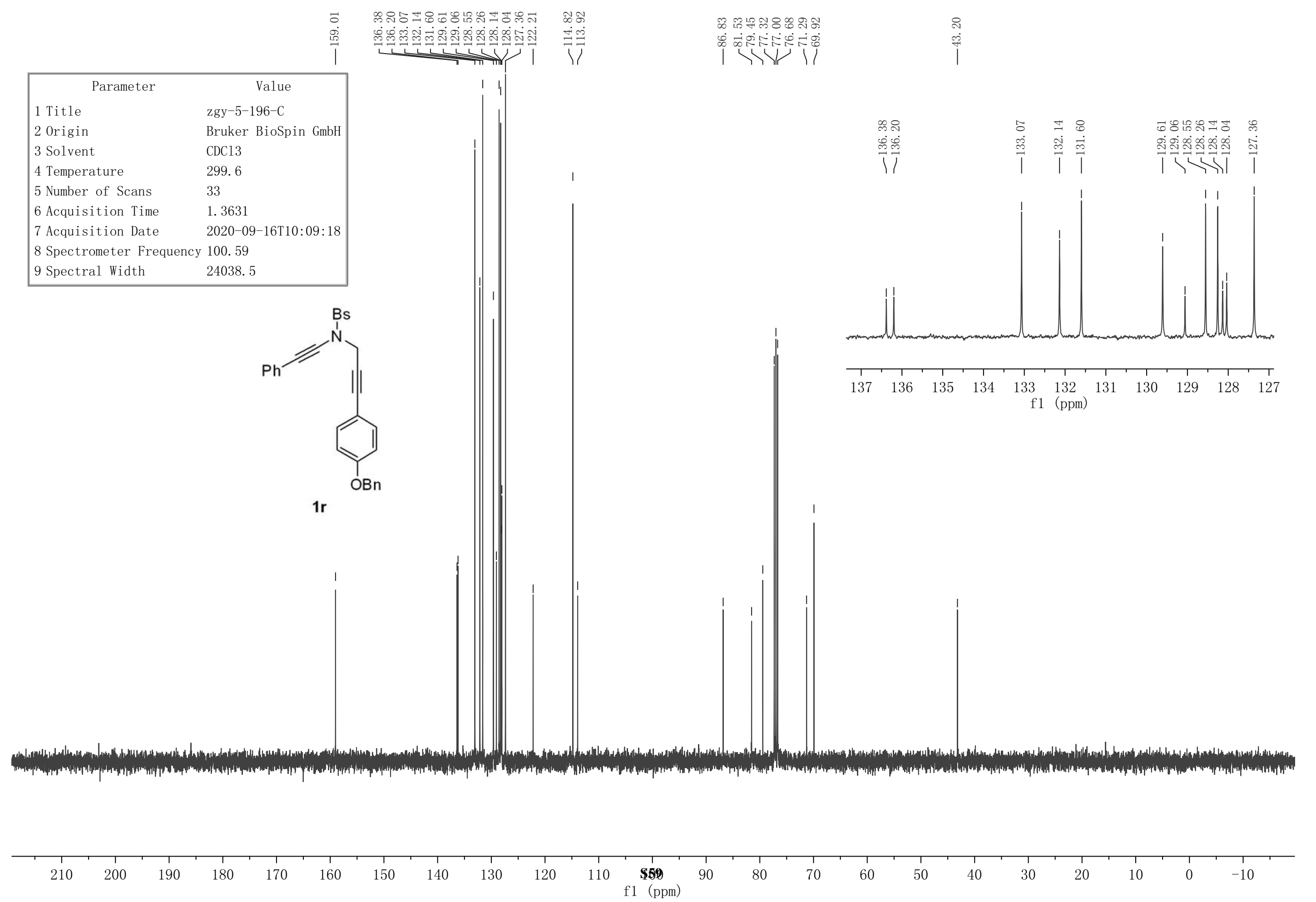


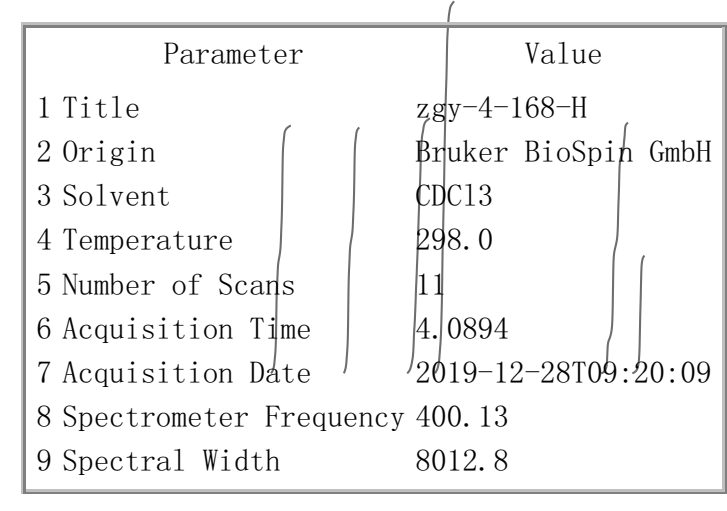
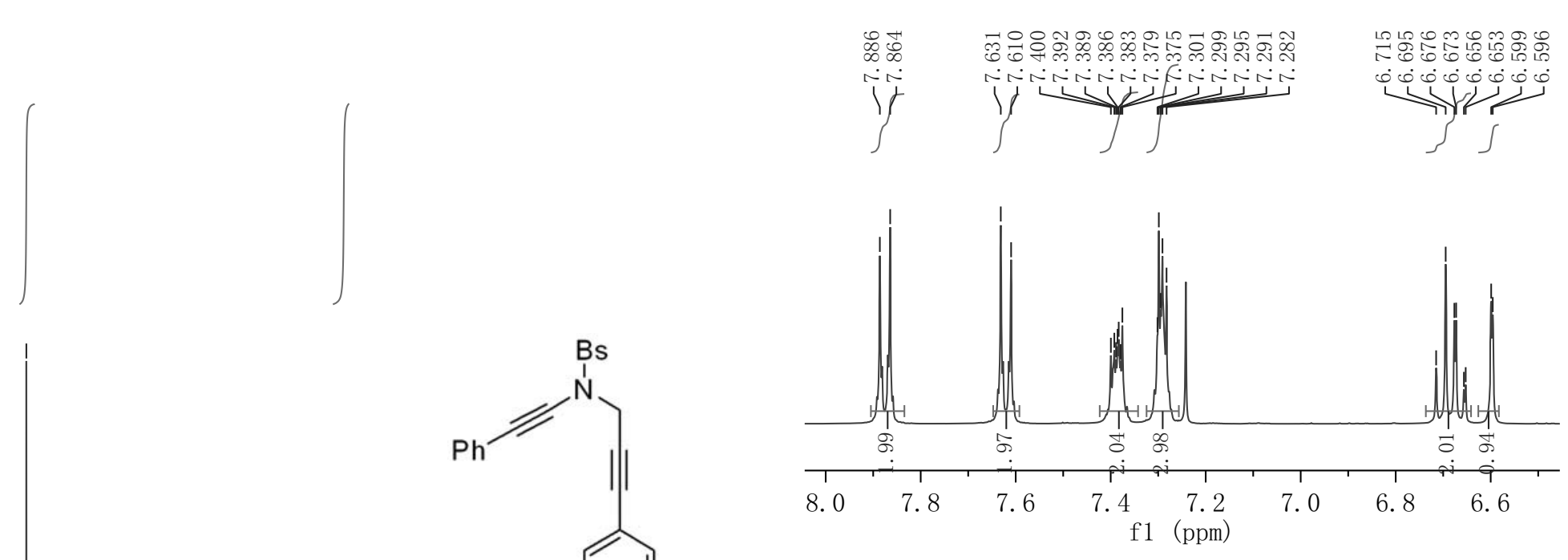

\begin{tabular}{|c|c|c|c|c|c|c|}
\hline & & 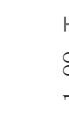 & \begin{tabular}{l}
$T$ \\
\multirow{2}{*}{} \\
-1
\end{tabular} & 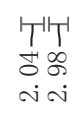 & & 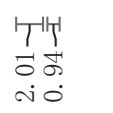 \\
\hline 0 & 8.5 & 8.0 & & & 7.0 & 6.5 \\
\hline
\end{tabular}

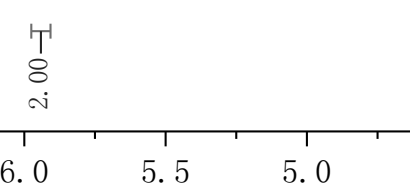
$\mathrm{T}^{\prime}$
8
i

\begin{tabular}{|c|c|c|c|c|c|c|c|c|c|c|}
\hline $\begin{array}{c}\mathbf{8 6 0} \\
\mathrm{f} 1 \quad \text { (ppm) }\end{array}$ & 3.5 & 3. 0 & 2.5 & 2.0 & 1.5 & 1.0 & 0.5 & 0.0 & -0.5 & -1 \\
\hline
\end{tabular}




\begin{tabular}{|ll|}
\hline \multicolumn{1}{|c|}{ Parameter } & \multicolumn{1}{c|}{ Value } \\
1 Title & zgy-4-168-C \\
2 Origin & Bruker BioSpin GmbH \\
3 Solvent & CDC13 \\
4 Temperature & 300.0 \\
5 Number of Scans & 20 \\
6 Acquisition Time & 1.3631 \\
7 Acquisition Date & $2019-12-28 T 09: 23: 30$ \\
8 Spectrometer Frequency & 100.61 \\
9 Spectral Width & 24038.5 \\
\hline
\end{tabular}
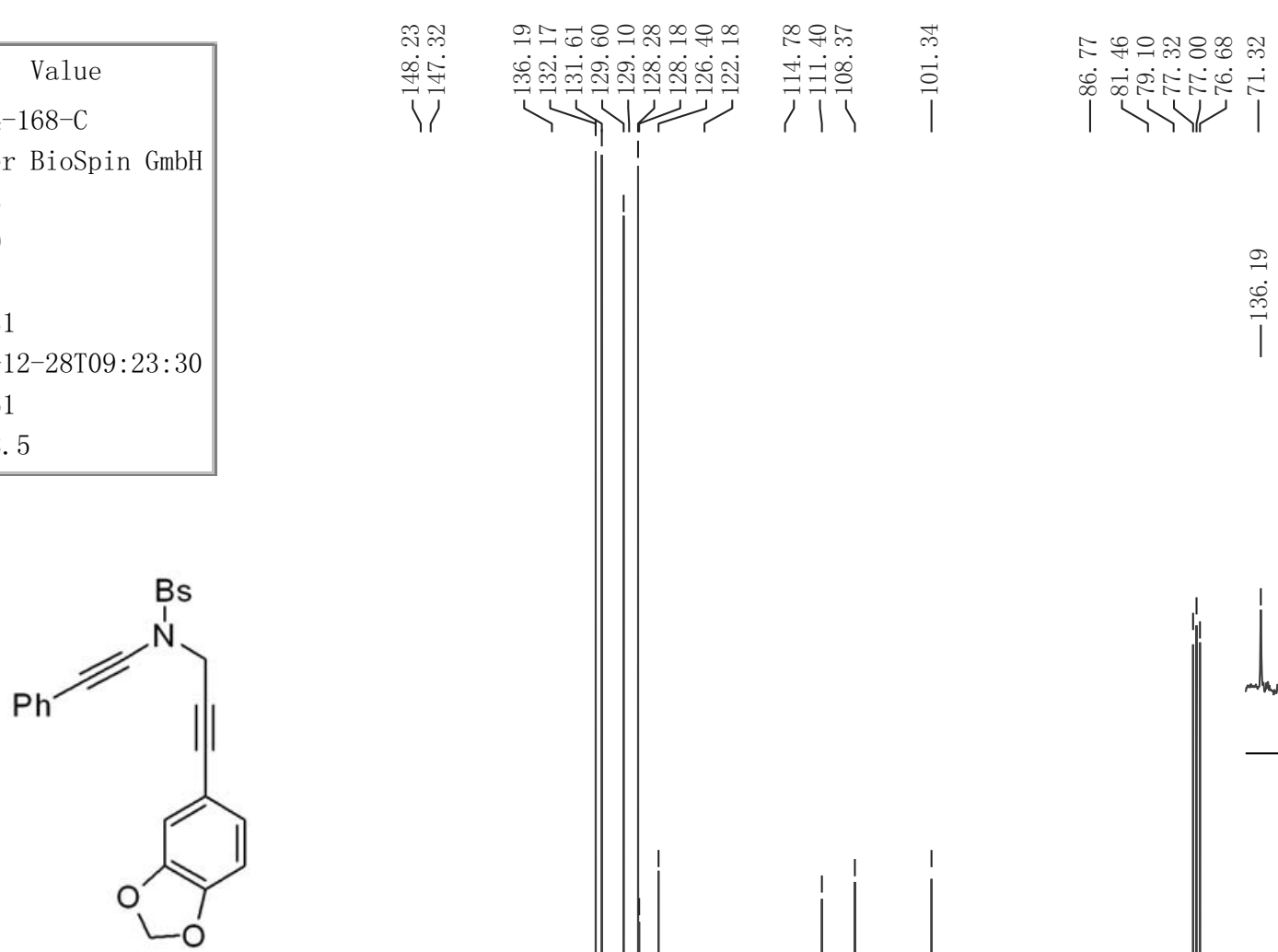

$1 \mathrm{~s}$

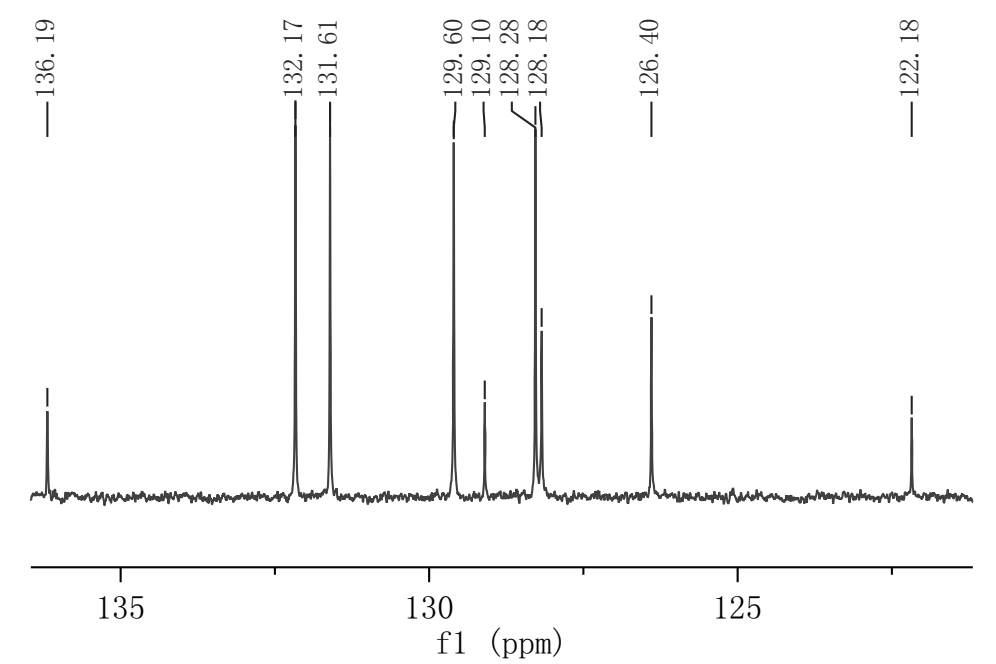

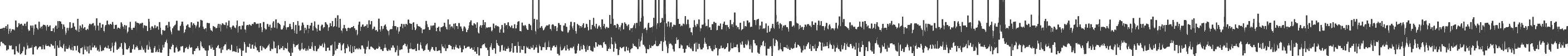

130

90

80

70

1
60

T 50

$\begin{array}{lllll}1 & 1 & 1 & 1 & 1 \\ 40 & 30 & 20 & 10 & 0\end{array}$




\begin{tabular}{|c|c|}
\hline Parameter & Value \\
\hline 1 Title & ZGY $-5-192-\mathrm{H}$ \\
\hline 2 Origin & Brukex BioSpin GmbH \\
\hline 3 Solvent & $\mathrm{CDCl} 3$ \\
\hline 4 Temperature & 298.0 \\
\hline 5 Number of Scans & 12 \\
\hline 6 Acquisition Time & 4. 0894 \\
\hline 7 Acquisition Date & 2020-09-08T17:09:38 \\
\hline \multicolumn{2}{|c|}{8 Spectrometer Frequency 400.13} \\
\hline 9 Spectral Width & 8012.8 \\
\hline
\end{tabular}
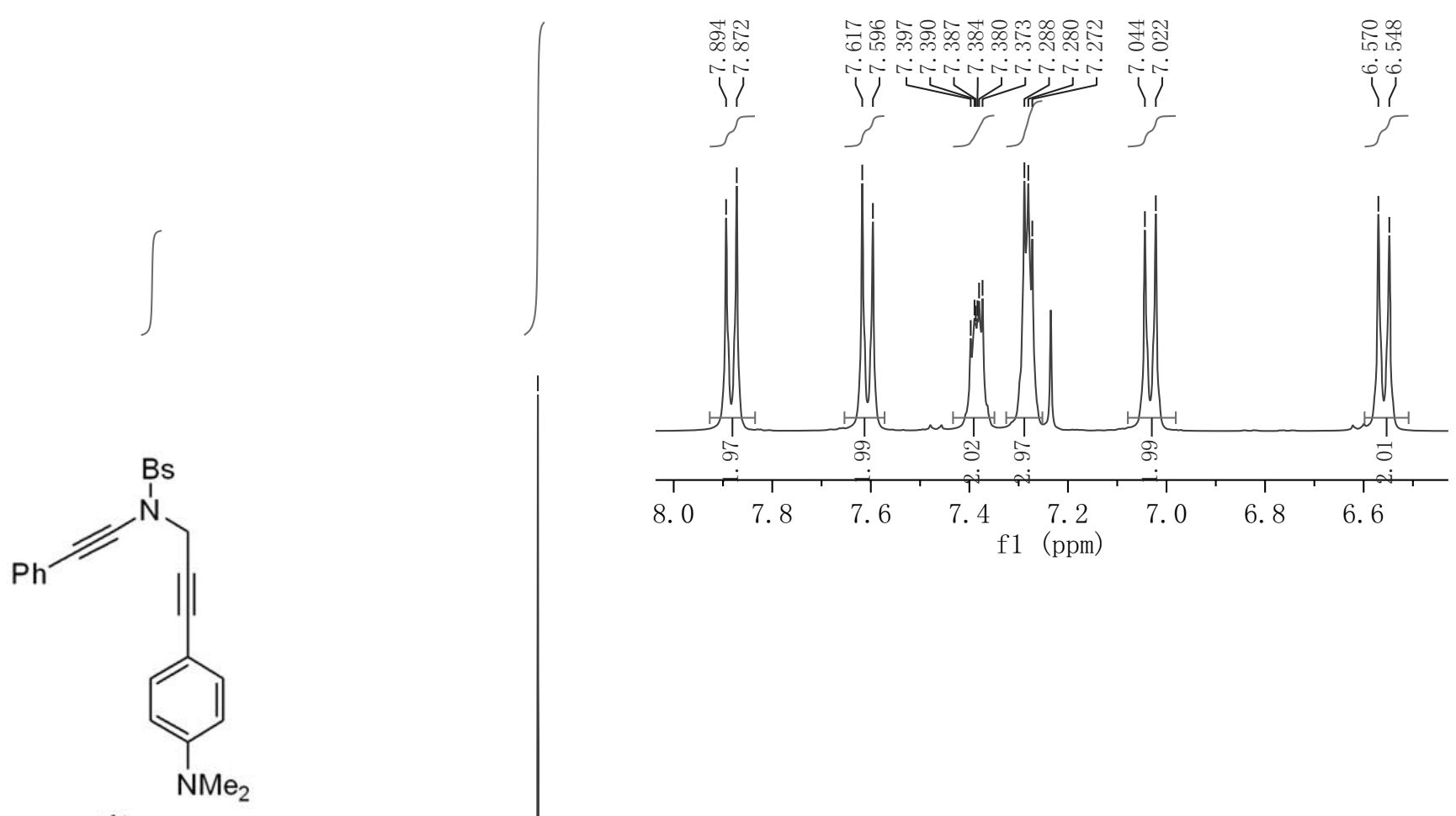

$1 t$
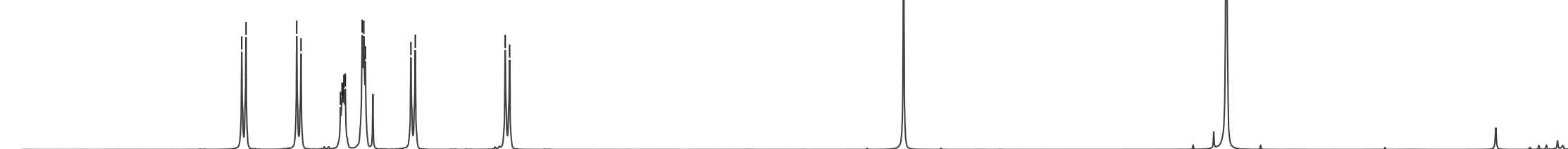

\begin{tabular}{|c|c|c|c|c|c|c|c|c|c|c|c|c|c|c|c|c|c|c|c|c|}
\hline & & 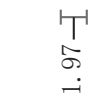 & 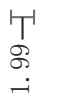 & 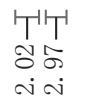 & 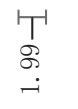 & $\begin{array}{l}T^{\top} \\
\overrightarrow{0} \\
\dot{N}\end{array}$ & & & & $\begin{array}{l}\text { 'T' } \\
\stackrel{1}{8} \\
\text { i }\end{array}$ & & & $\begin{array}{l}\text { 'T' } \\
8 \\
8 \\
0\end{array}$ & & & & & & & \\
\hline 0 & 8.5 & 8.0 & 7. & & 7. 0 & 6.5 & 6. 0 & 5.5 & 5.0 & 4.5 & $\begin{array}{l}\mathbf{8 6 2} \\
1 \quad(\mathrm{ppm})\end{array}$ & 3.5 & 3. 0 & 2.5 & 2.0 & 1.5 & 1.0 & 0.5 & 0.0 & -0.5 \\
\hline
\end{tabular}




\begin{tabular}{|ll|}
\hline \multicolumn{1}{|c|}{ Parameter } & \multicolumn{1}{c|}{ Value } \\
1 Title & ZGY-5-192-C \\
2 Origin & Bruker BioSpin GmbH \\
3 Solvent & CDC13 \\
4 Temperature & 300.0 \\
5 Number of Scans & 36 \\
6 Acquisition Time & 1.3631 \\
7 Acquisition Date & $2020-09-08 T 17: 13: 11$ \\
8 Spectrometer Frequency 100.61 \\
9 Spectral Width & 24038.5 \\
\hline
\end{tabular}

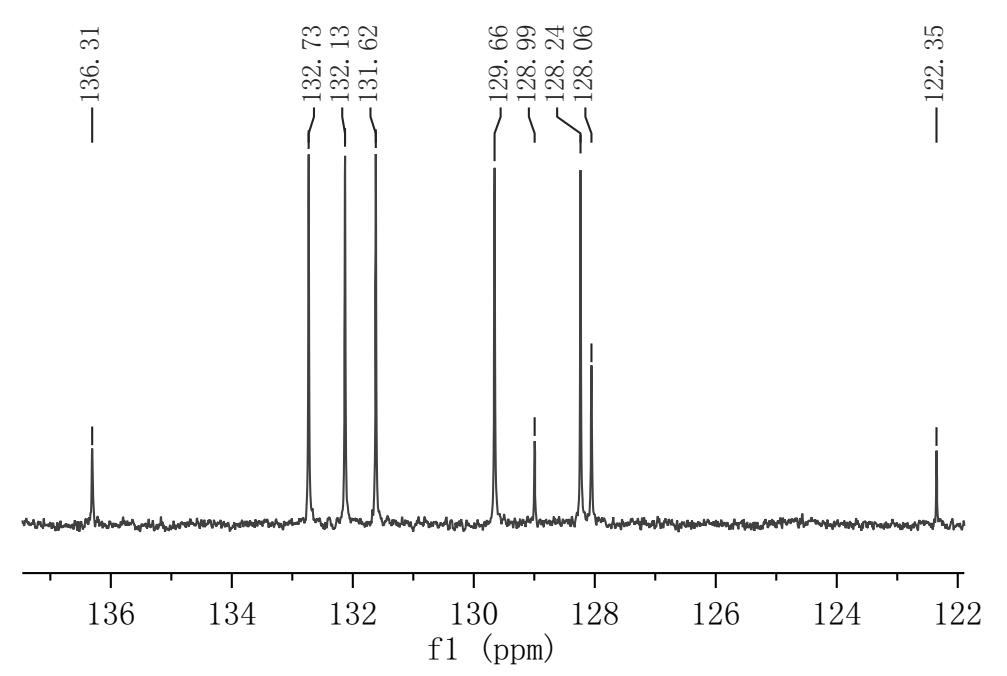

$1 t$
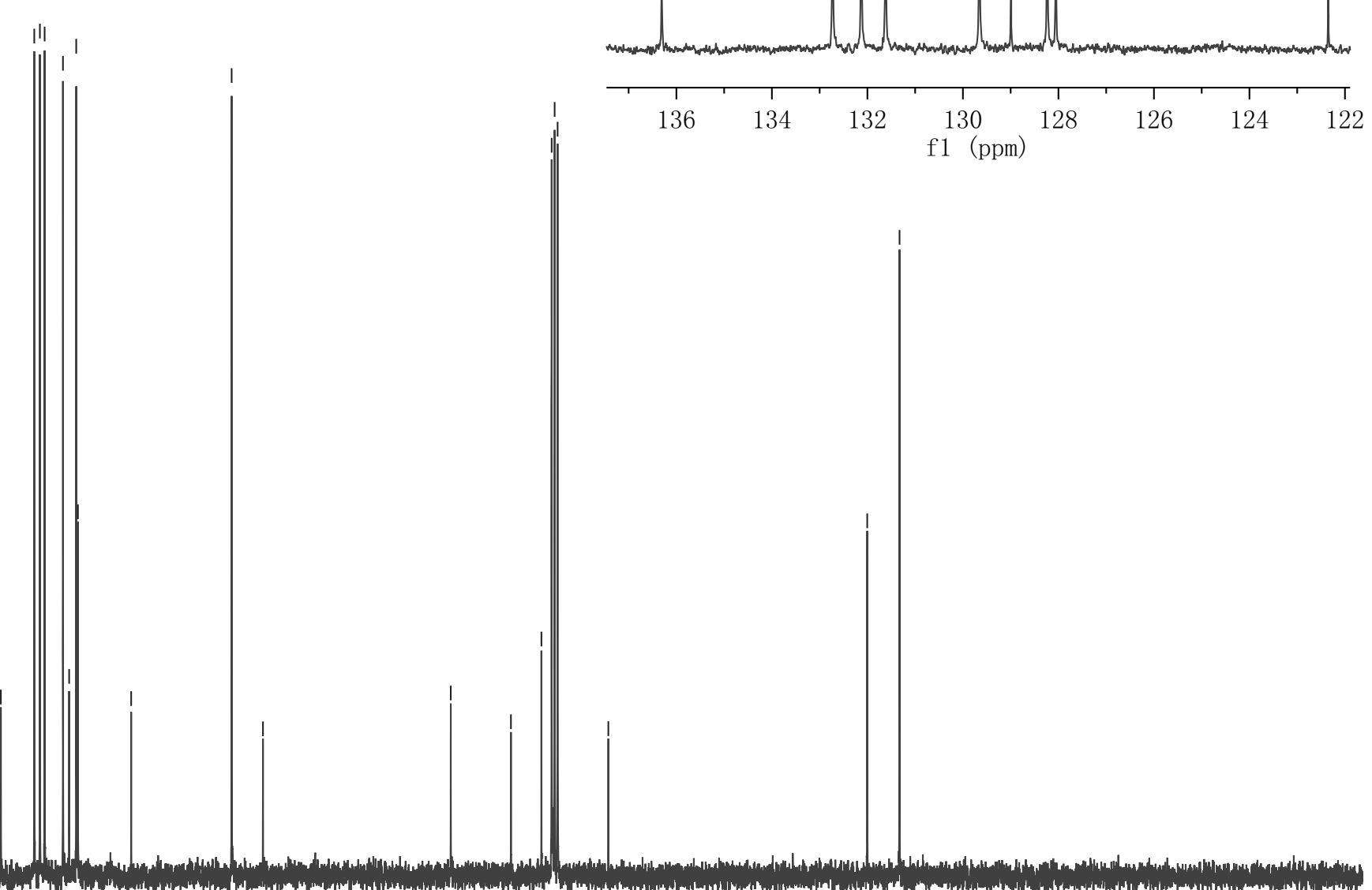

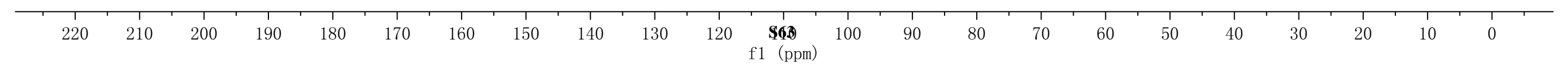




\begin{tabular}{|ll|}
\hline \multicolumn{1}{|c|}{ Parameter } & \multicolumn{1}{c|}{ Value } \\
1 Title & zgy-4-148-H \\
2 Origin & Bruker BioSpin GmbH \\
3 Solvent & CDC13 \\
4 Temperature & 298.0 \\
5 Number of Scans & $1.5 \mid$ \\
6 Acquisition Time/ & 4.10894 \\
7 Acquisition Date & $2019-12-23 T 10: 22: 34$ \\
8 Spectrometer Frequency 400.13 \\
9 Spectral Width & 8012.8 \\
\hline
\end{tabular}

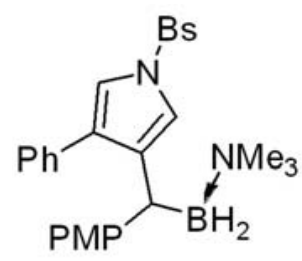

2a
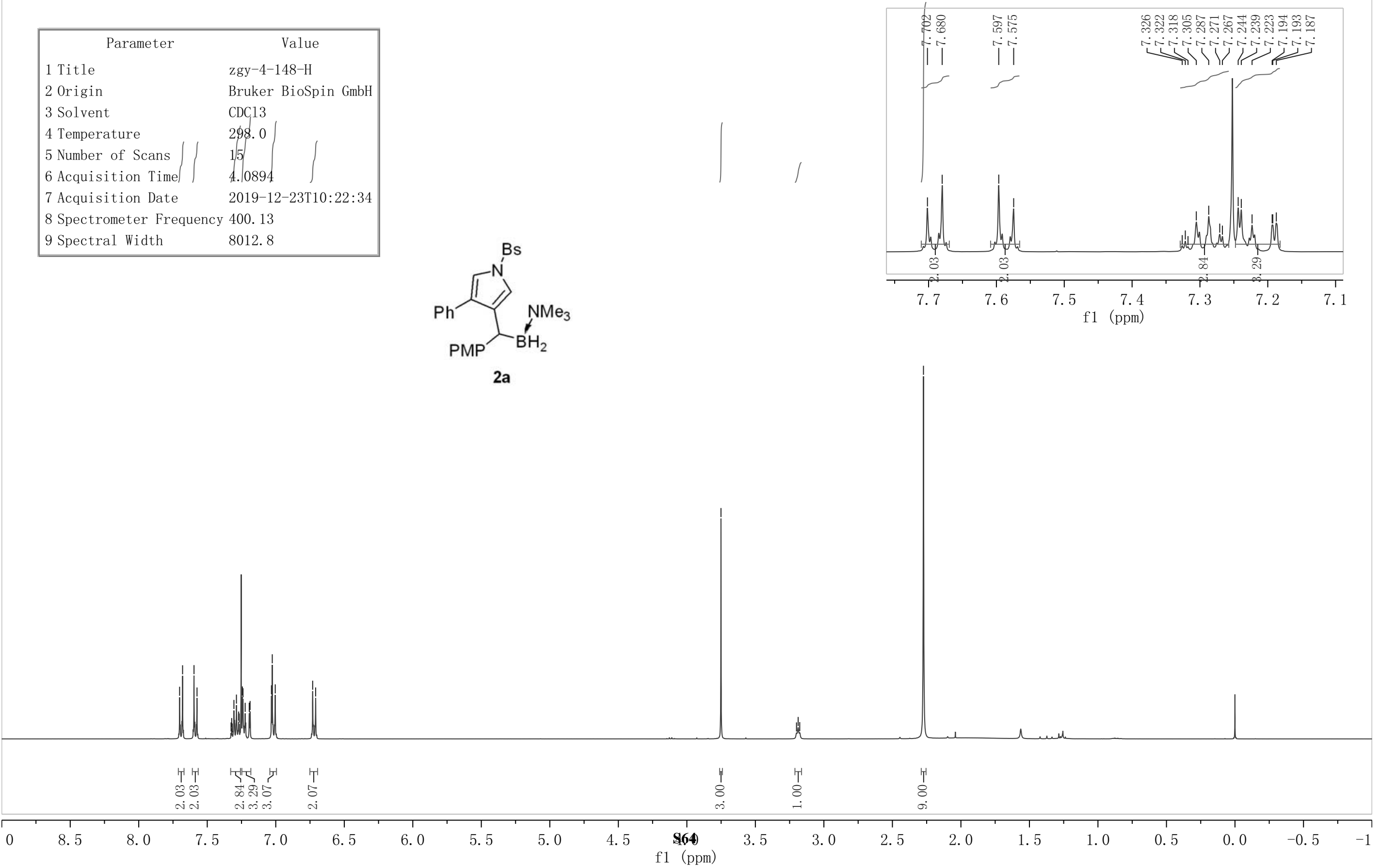


\begin{tabular}{|ll|}
\hline \multicolumn{1}{|c|}{ Parameter } & \multicolumn{1}{c|}{ Value } \\
1 Title & zgy-4-148-C \\
2 Origin & Bruker BioSpin GmbH \\
3 Solvent & CDC13 \\
4 Temperature & 300.0 \\
5 Number of Scans & 93 \\
6 Acquisition Time & 1.3631 \\
7 Acquisition Date & $2019-12-23 T 10: 27: 41$ \\
8 Spectrometer Frequency 100.61 \\
9 Spectral Width & 24038.5 \\
\hline
\end{tabular}
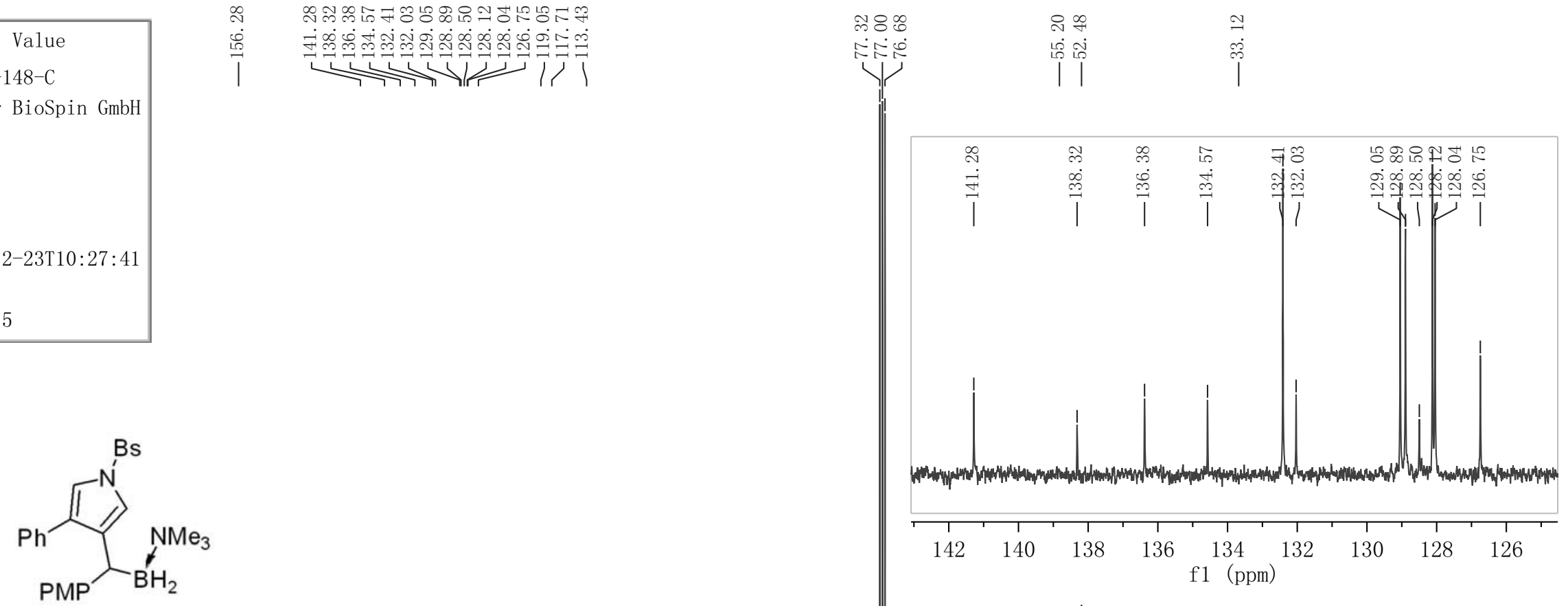

$2 a$
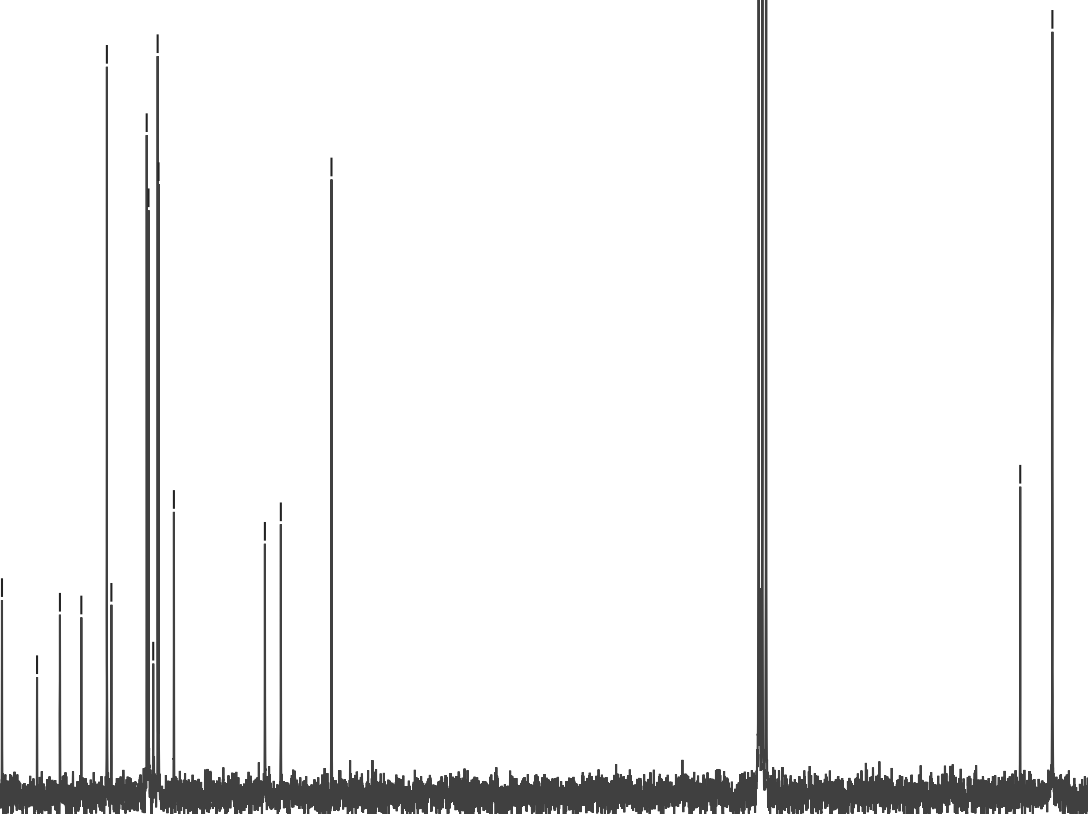

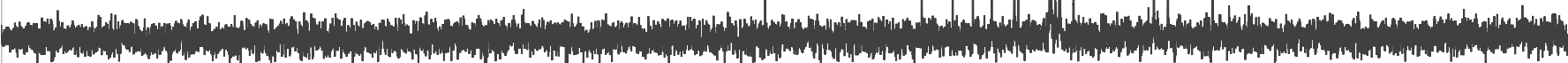

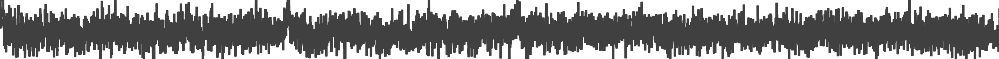

\section{\$65)}

1009

$90 \quad 80$

$80 \quad 70$

$70 \quad 60$

$60 \quad 50$

$50 \quad 4$

$40 \quad 30$

30




\begin{tabular}{|ll|}
\hline \multicolumn{1}{|c|}{ Parameter } & \multicolumn{1}{c|}{ Value } \\
1 Title & zgy-4-148-C-dept \\
2 Origin & Bruker BioSpin GmbH \\
3 Solvent & CDC13 \\
4 Temperature & 300.0 \\
5 Number of Scans & 65 \\
6 Acquisition Time & 1.2976 \\
7 Acquisition Date & $2019-12-23 \mathrm{~T} 10: 33: 19$ \\
8 Spectrometer Frequency 100.61 \\
9 Spectral Width & 25252.5 \\
\hline
\end{tabular}

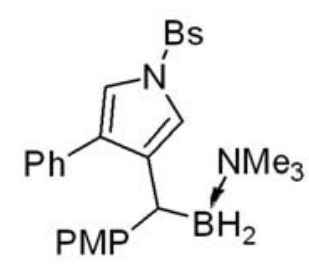

2a

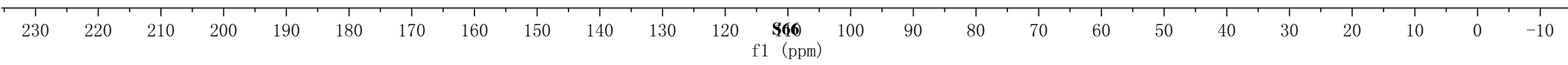




\begin{tabular}{|c|c|}
\hline Parameter & Value \\
\hline 1 Title & zgy $-4-120-\mathrm{H}$ \\
\hline 2 Origin & Bruker BioSpin GmbH \\
\hline 3 Solvent & CDC13 \\
\hline 4 Temperature & 298.0 \\
\hline 5 Number of Scans & 11 \\
\hline 6 Acquisition Time & 4. 0894 \\
\hline 7 Acquisition Date & $2019-12-06 \mathrm{~T} 21: 26: 48$ \\
\hline \multicolumn{2}{|c|}{8 Spectrometer Frequency 400.13 } \\
\hline 9 Spectral Width & 8012.8 \\
\hline
\end{tabular}
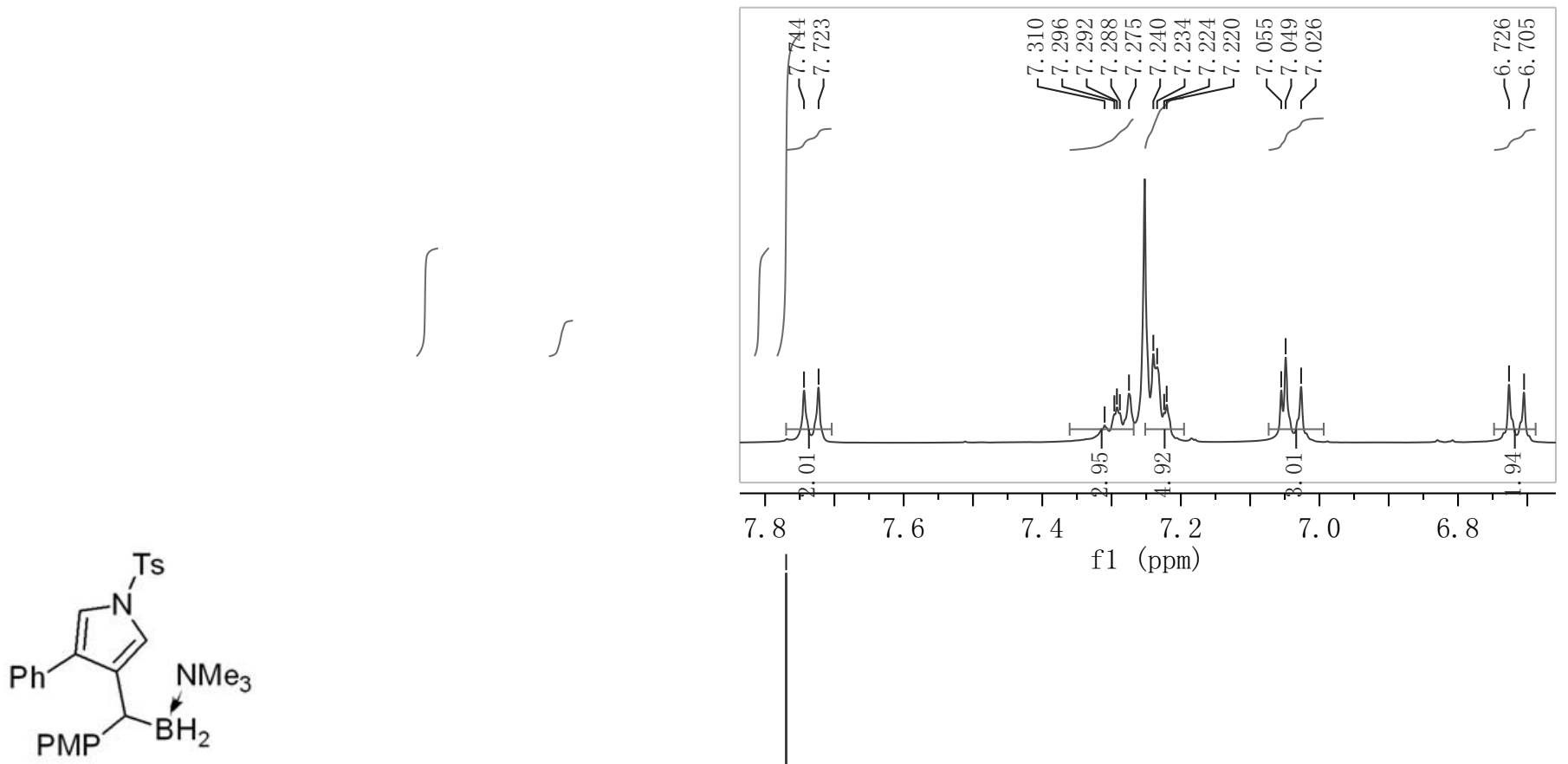

2b

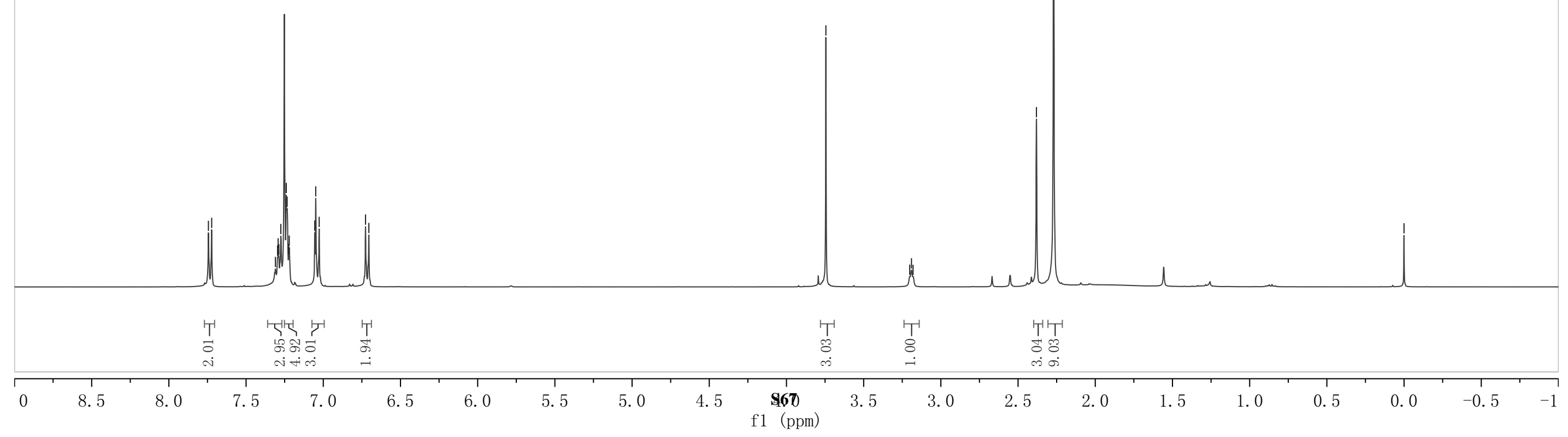




\begin{tabular}{|ll|}
\hline \multicolumn{1}{|c|}{ Parameter } & \multicolumn{1}{c|}{ Value } \\
1 Title & zgy-4-120-C \\
2 Origin & Bruker BioSpin GmbH \\
3 Solvent & CDC13 \\
4 Temperature & 300.0 \\
5 Number of Scans & 56 \\
6 Acquisition Time & 1.3631 \\
7 Acquisition Date & $2019-12-06 \mathrm{~T} 21: 31: 16$ \\
8 Spectrometer Frequency 100.61 \\
9 Spectral Width & 24038.5 \\
\hline
\end{tabular}

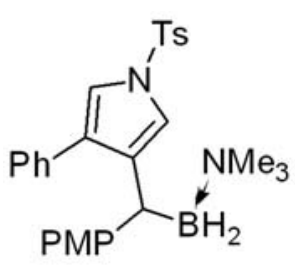

$2 b$
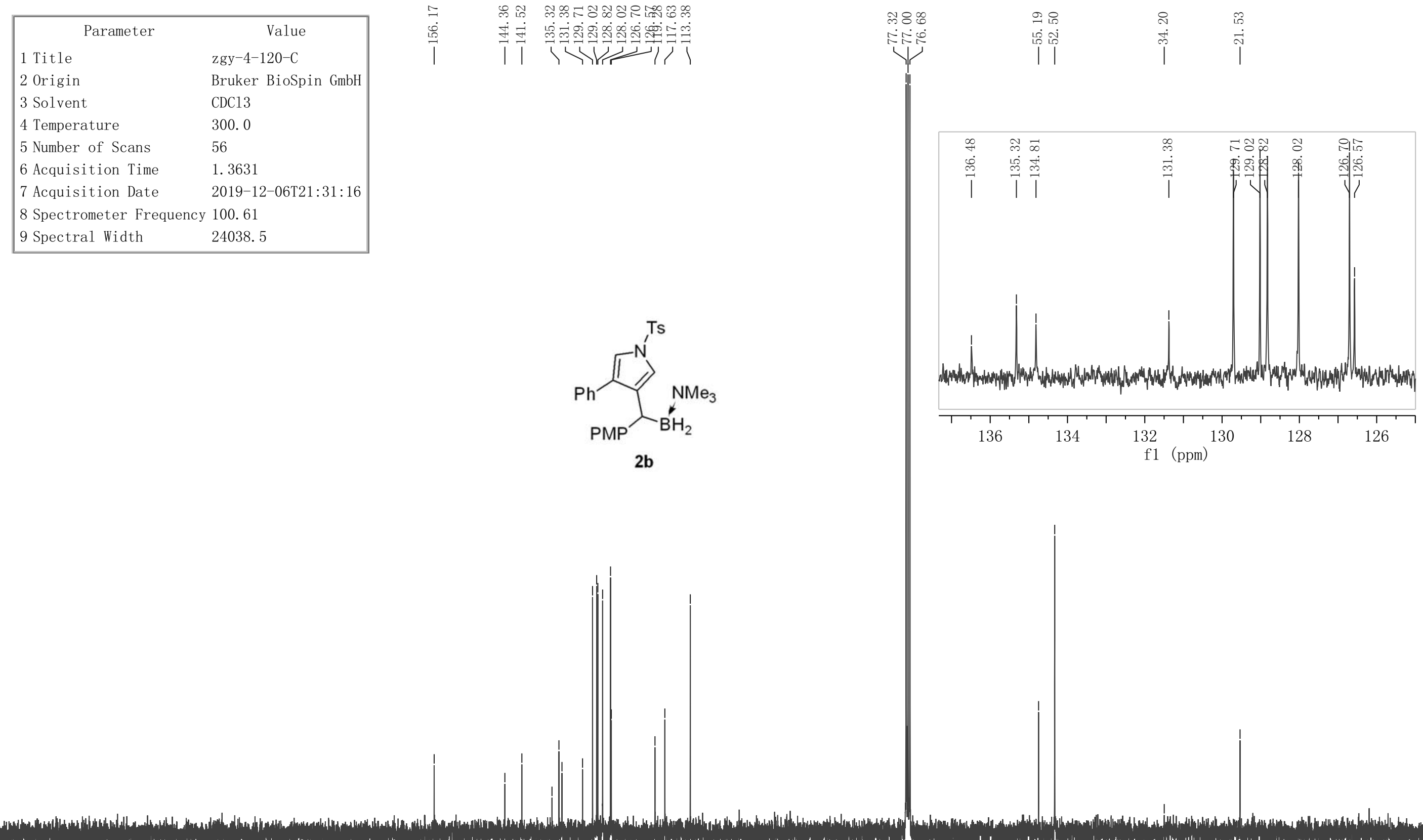

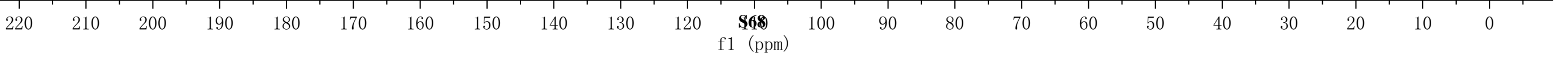




\begin{tabular}{|ll|}
\hline \multicolumn{1}{|c|}{ Parameter } & \multicolumn{1}{c|}{ Value } \\
1 Title & zgy-4-121-H \\
2 Origin & Bruker BioSpin GmbH \\
3 Solvent & CDC13 \\
4 Temperature & 298.0 \\
5 Number of Scans & $10 \iint_{6} \int$ \\
6 Acquisition Time & 4.0894 \\
7 Acquisition Date & $2019-12-06 \mathrm{~T} 17: 47: 36$ \\
8 Spectrometer Frequency & 400.13 \\
9 Spectral Width & 8012.8 \\
\hline
\end{tabular}
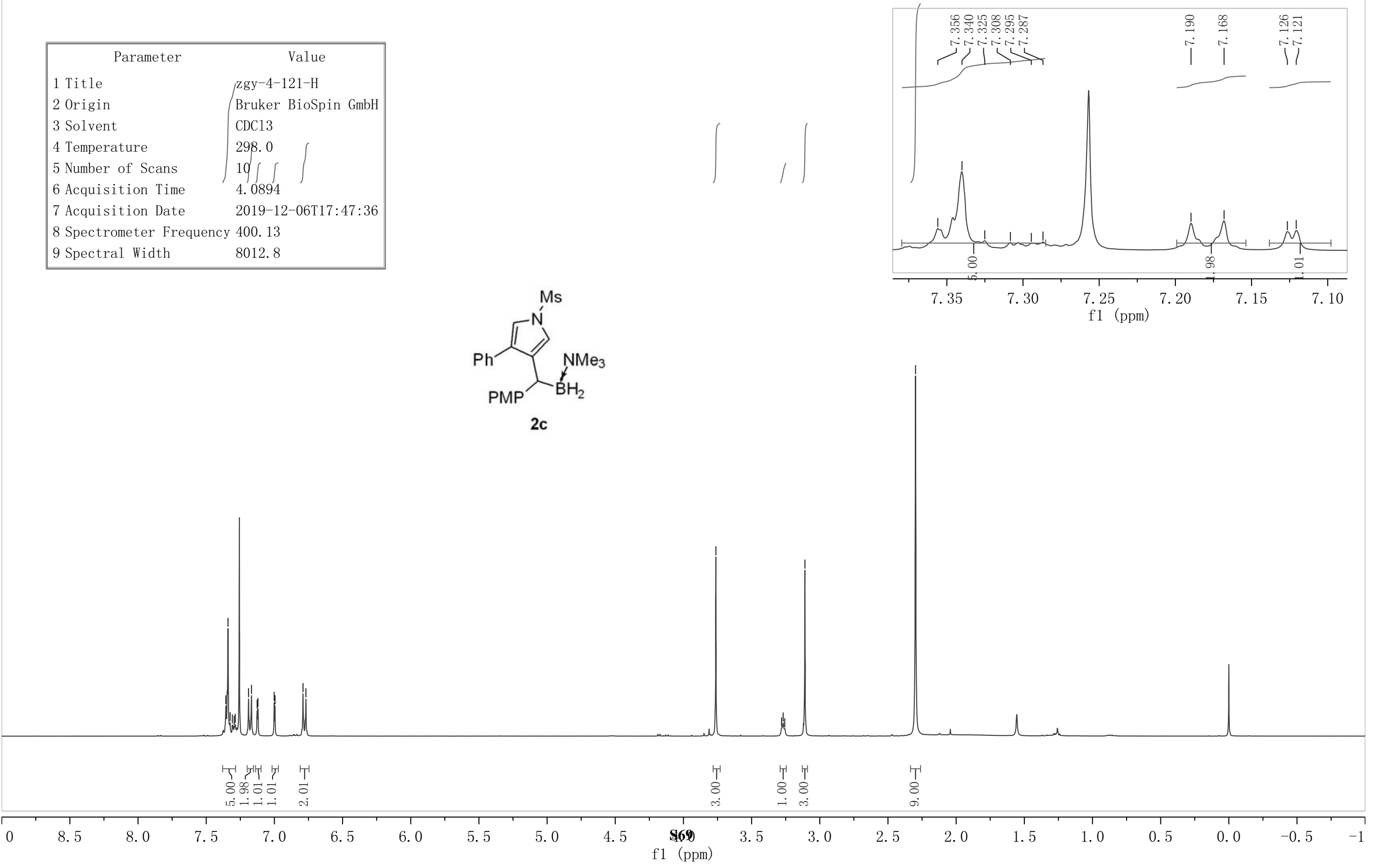


\begin{tabular}{|ll|}
\hline \multicolumn{1}{|c|}{ Parameter } & \multicolumn{1}{c|}{ Value } \\
1 Title & zgy-4-121-C \\
2 Origin & Bruker BioSpin GmbH \\
3 Solvent & CDC13 \\
4 Temperature & 300.0 \\
5 Number of Scans & 120 \\
6 Acquisition Time & 1.3631 \\
7 Acquisition Date & $2019-12-06 T 17: 50: 26$ \\
8 Spectrometer Frequency 100.61 \\
9 Spectral Width & 24038.5 \\
\hline
\end{tabular}

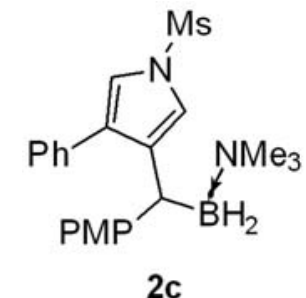

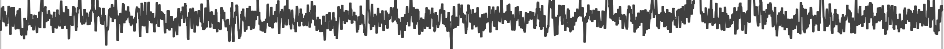

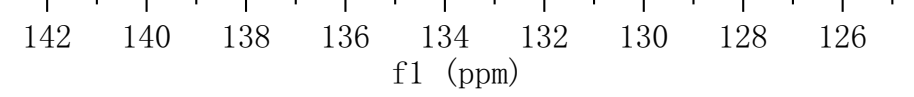

2c

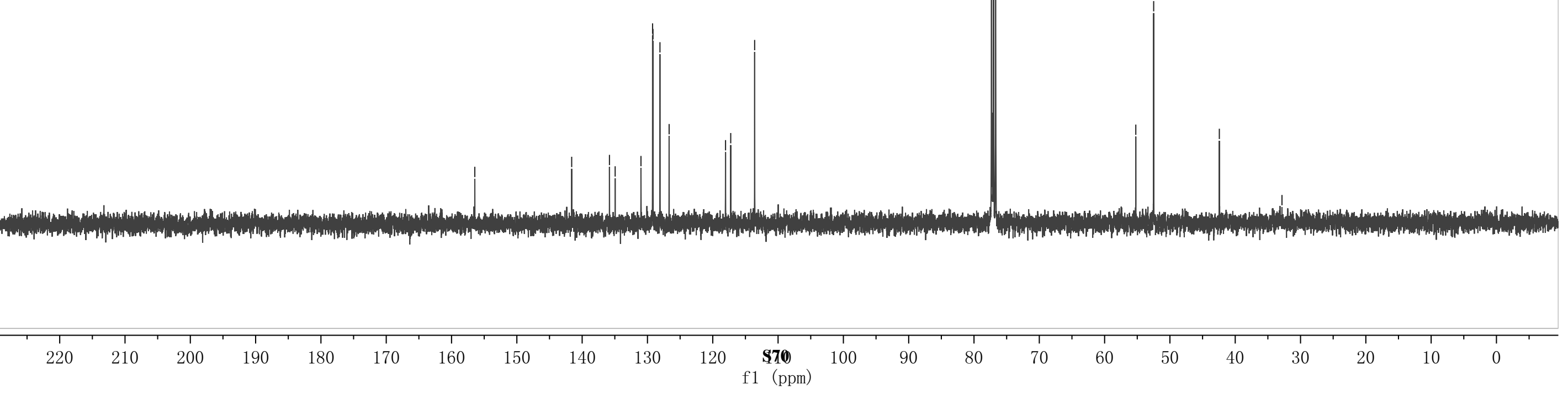




\begin{tabular}{|ll|}
\hline \multicolumn{1}{|c|}{ Parameter } & \multicolumn{1}{c|}{ Value } \\
1 Title & zgy-4-166-H \\
2 Origin & Bruker BioSpin GmbH \\
3 Solvent & CDC13 \\
4 Temperature & 298.0 \\
5 Number of Scans & 12 \\
6 Acquisition Time & 4.0894 \\
7 Acquisition Date & $2019-12-\$ 1 \mathrm{~T} 10: 14: 40$ \\
8 Spectrometer Frequency & 400.13 \\
9 Spectral Width & 8012.8 \\
\hline
\end{tabular}
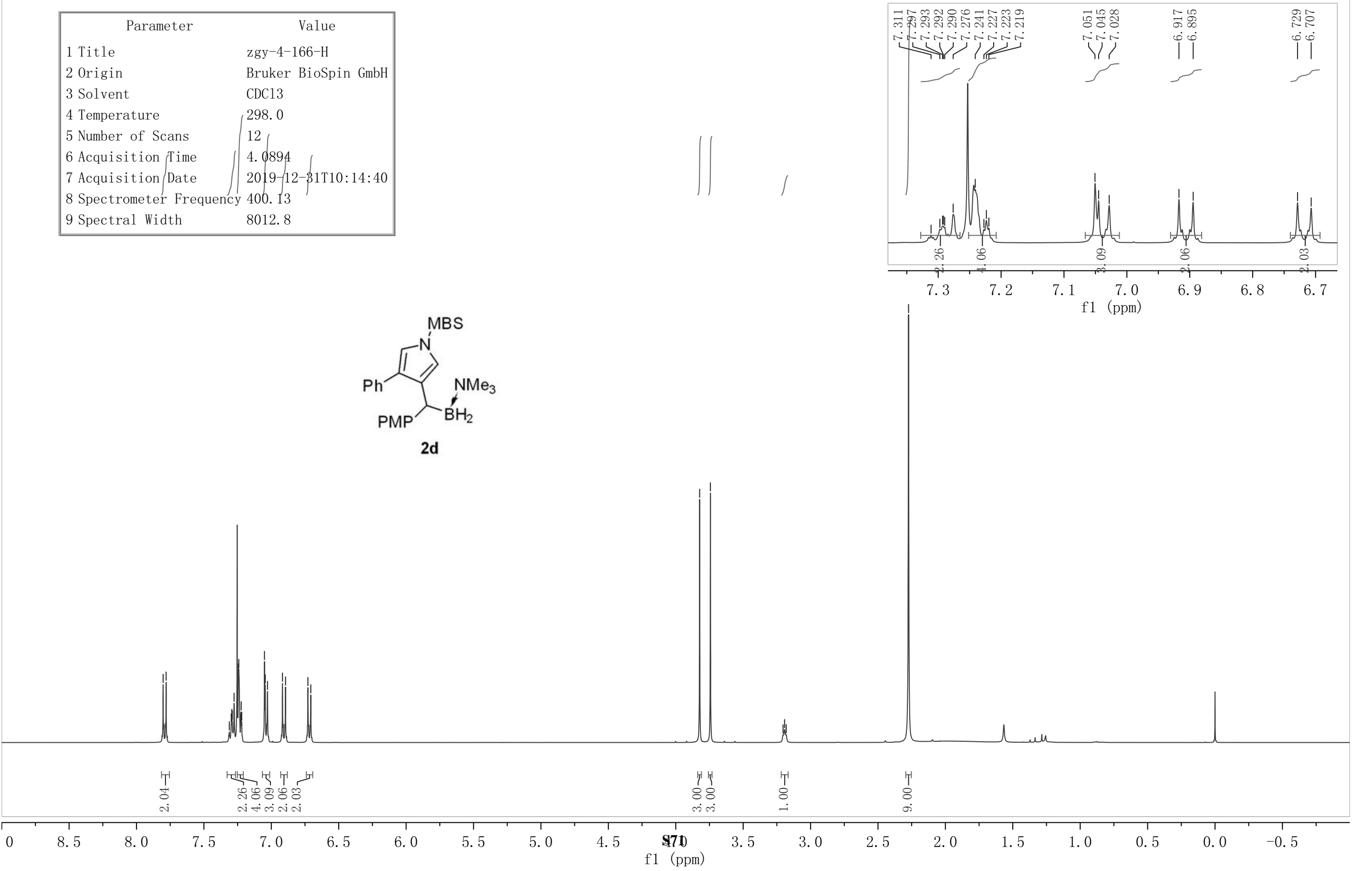


\begin{tabular}{|ll|}
\hline \multicolumn{1}{|c|}{ Parameter } & \multicolumn{1}{c|}{ Value } \\
1 Title & zgy-4-166-C \\
2 Origin & Bruker BioSpin GmbH \\
3 Solvent & CDC13 \\
4 Temperature & 300.0 \\
5 Number of Scans & 59 \\
6 Acquisition Time & 1.3631 \\
7 Acquisition Date & $2019-12-31$ T10:17:41 \\
8 Spectrometer Frequency 100.61 \\
9 Spectral Width & 24038.5 \\
\hline
\end{tabular}
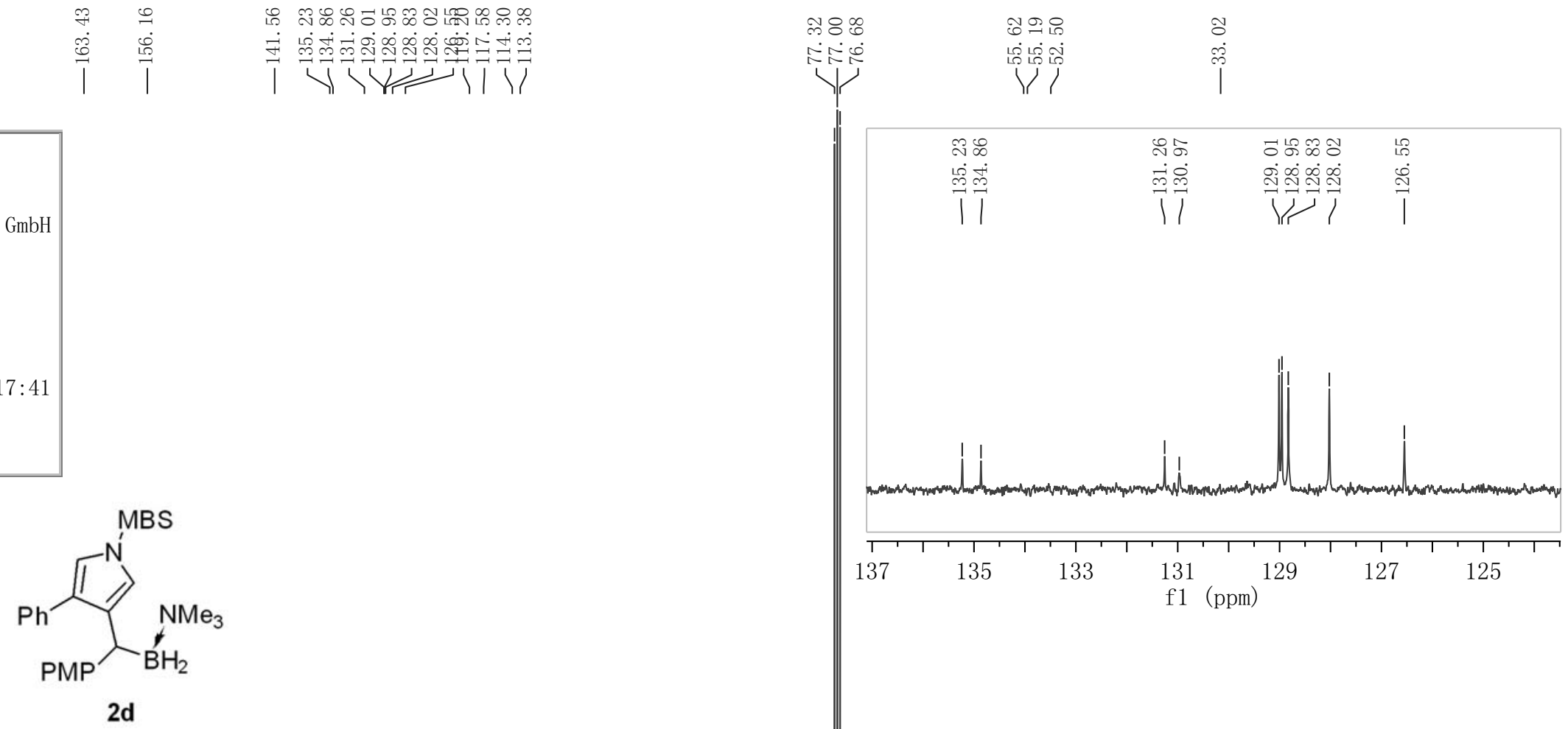

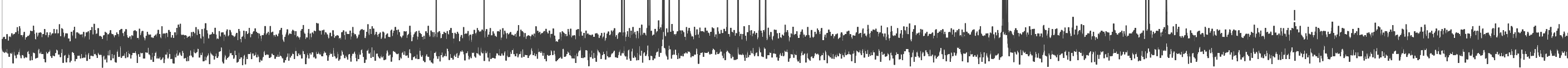




\begin{tabular}{|c|c|}
\hline Parameter & Value \\
\hline 1 Title & zgy-4-134-H \\
\hline 2 Origin & Bruker BioSpin GmbH \\
\hline 3 Solvent & $\mathrm{CDCl3}$ \\
\hline 4 Temperature & 298.0 \\
\hline 5 Number of Scans & 9 \\
\hline 6 Acquisition Tíme & 4. 0894 \\
\hline 7 Acquisition Date & $2019-12-13 T 14: 34: 33$ \\
\hline 8 Spectrometer Freq & 400.13 \\
\hline 9 Spectral Width & 8012.8 \\
\hline
\end{tabular}
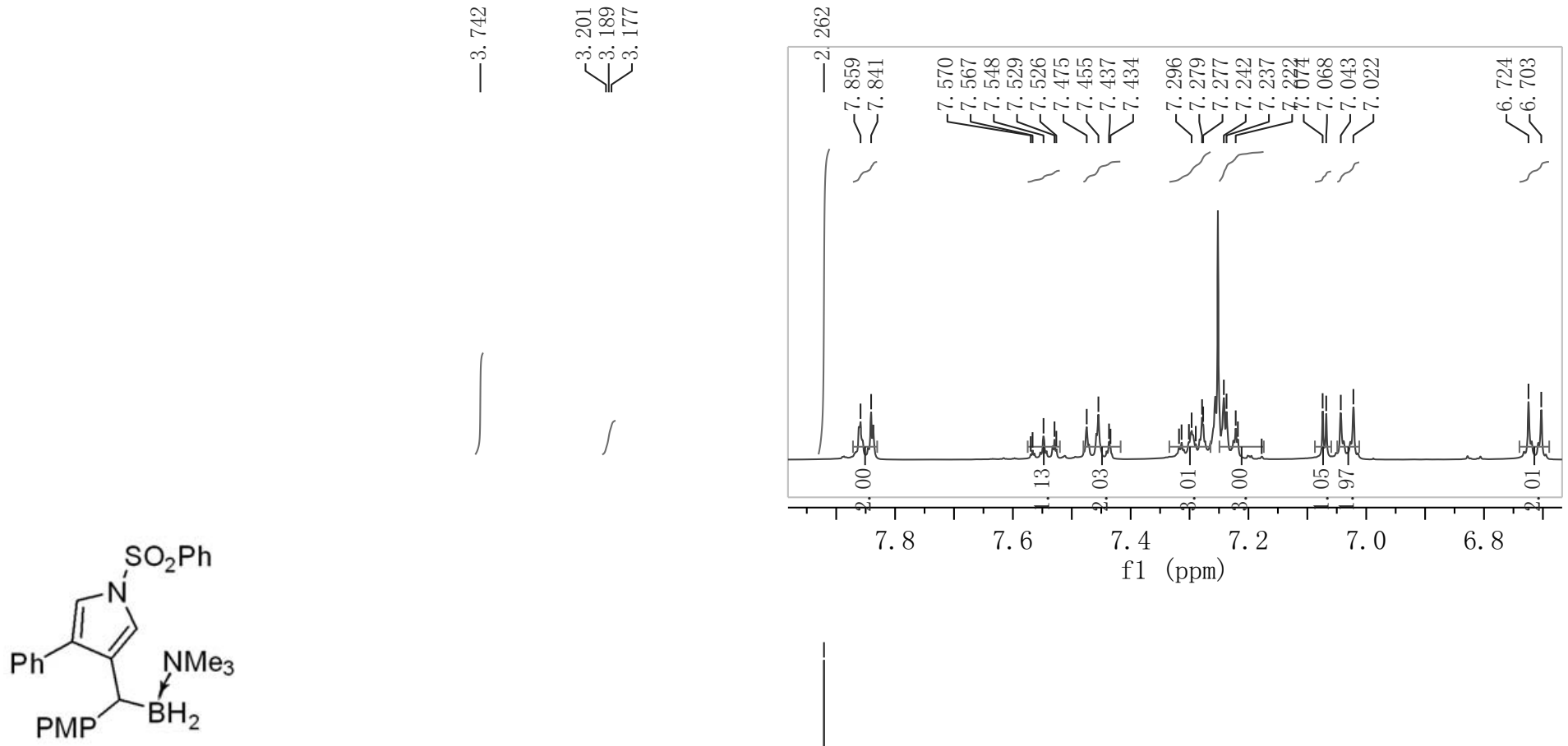

$2 \mathrm{e}$

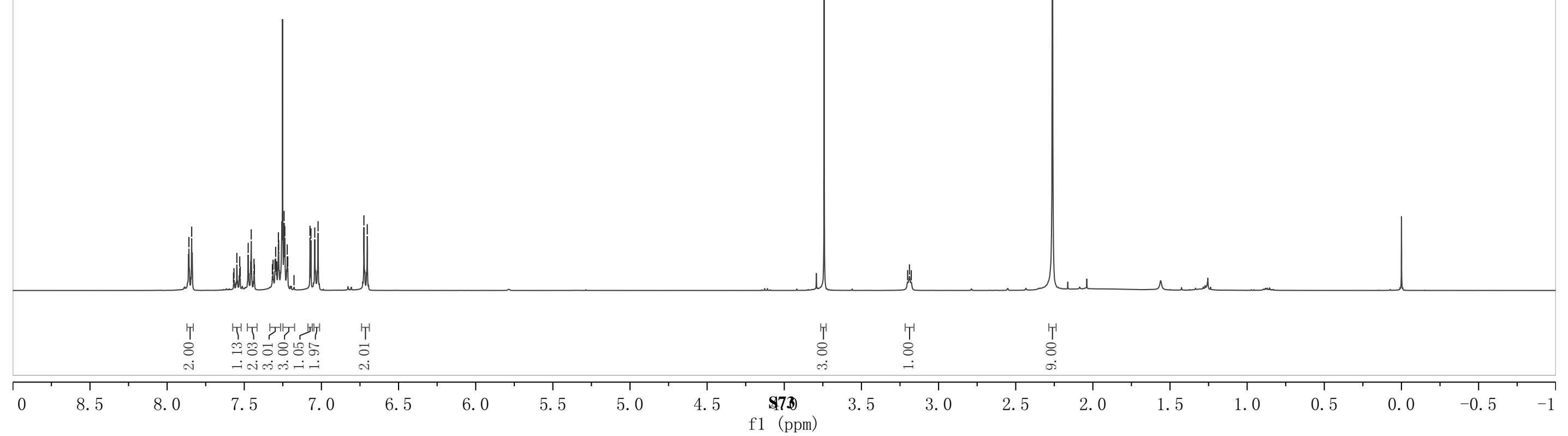




\begin{tabular}{|ll|}
\hline \multicolumn{1}{|c|}{ Parameter } & \multicolumn{1}{c|}{ Value } \\
1 Title & zgy-4-134-C \\
2 Origin & Bruker BioSpin GmbH \\
3 Solvent & CDC13 \\
4 Temperature & 300.0 \\
5 Number of Scans & 130 \\
6 Acquisition Time & 1.3631 \\
7 Acquisition Date & $2019-12-13 T 09: 14: 06$ \\
8 Spectrometer Frequency 100.61 \\
9 Spectral Width & 24038.5 \\
\hline
\end{tabular}
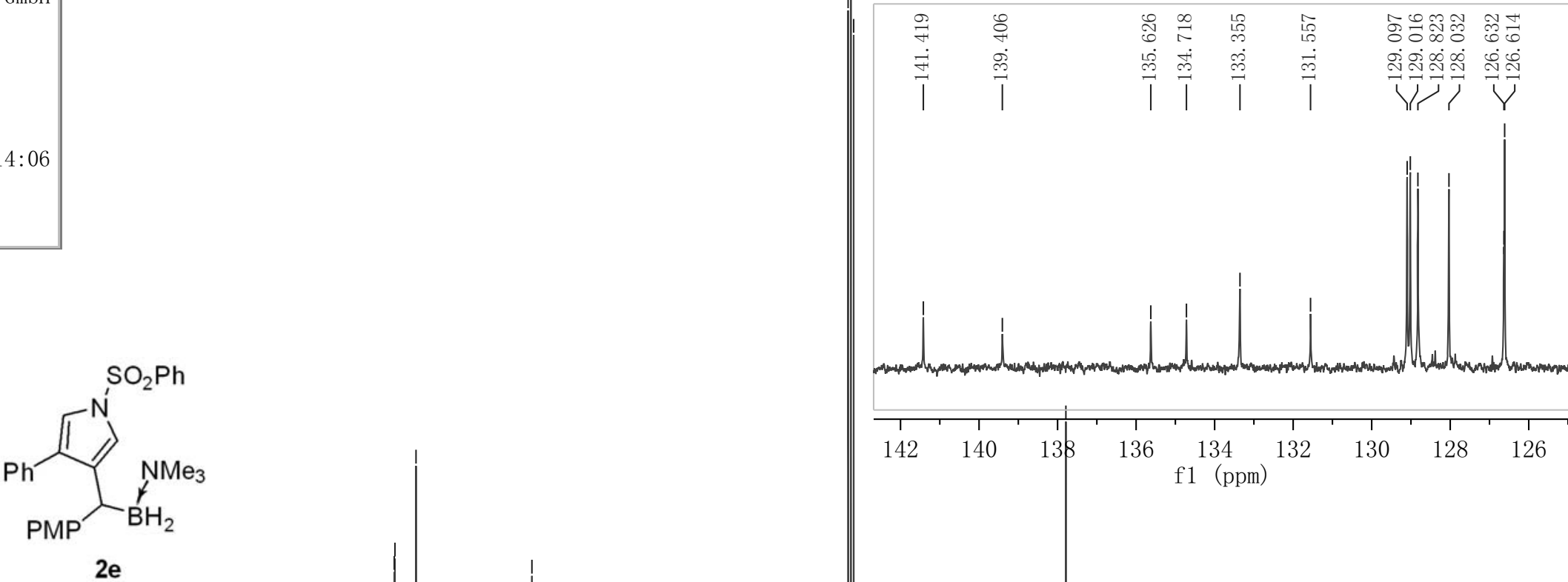

$2 e$

$90 \quad 80$

70

60

50




\begin{tabular}{|c|c|}
\hline Parameter & Value \\
\hline 1 Title & zgy-4-113-H \\
\hline 2 Origin & Bruker BioSpin GmbH \\
\hline 3 Solvent & $\operatorname{cDCl} 3$ \\
\hline 4 Temperature & $29 \$ .0$ \\
\hline 5 Number of Scans & 11 \\
\hline 6 Acquisition Time & 4. 0894 \\
\hline 7 Acquisition Date & 2020-01-04Т09:37:50 \\
\hline 8 Spectrometer Freq & 400.13 \\
\hline 9 Spectral Width & 8012.8 \\
\hline
\end{tabular}
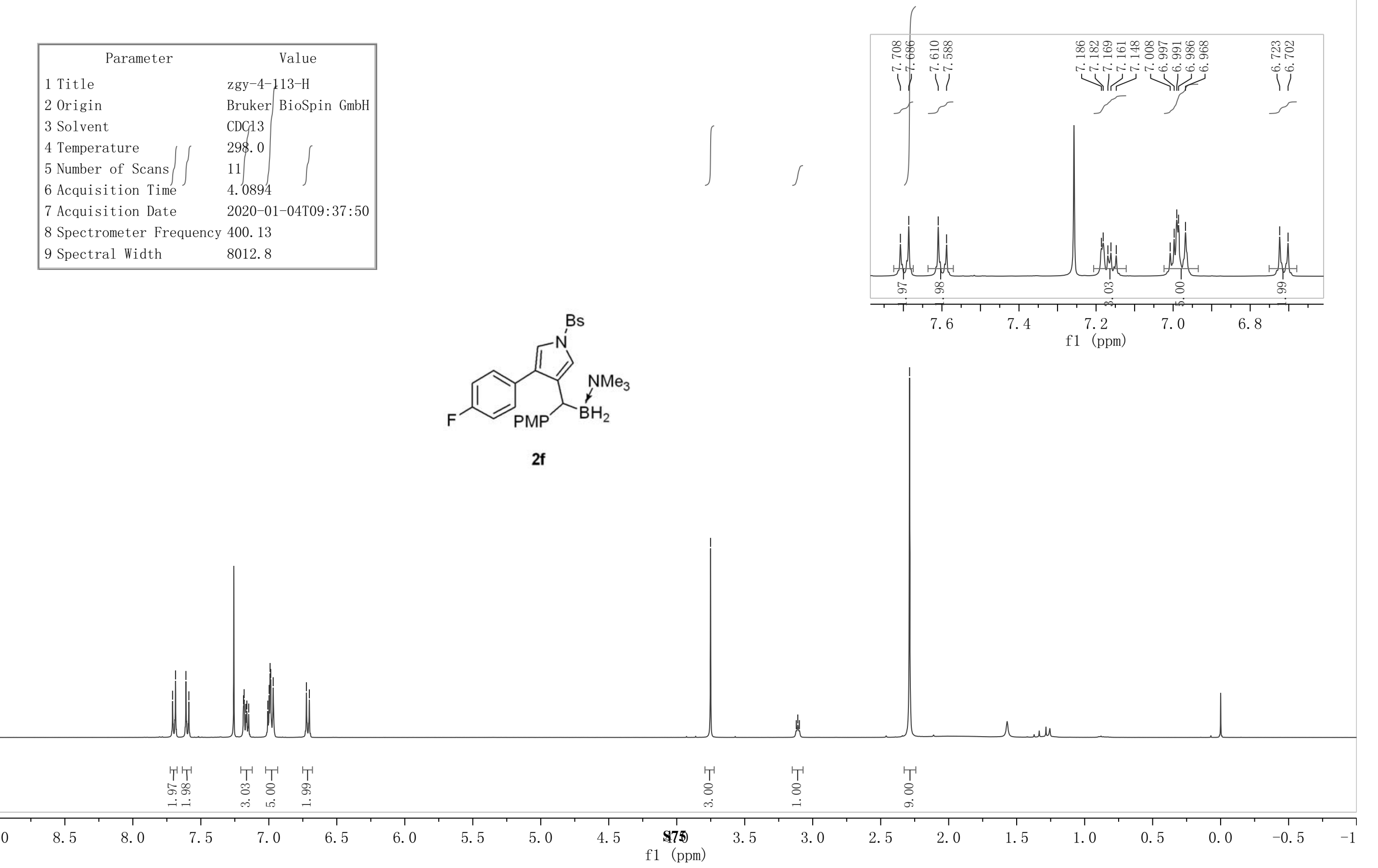


\begin{tabular}{|ll|}
\hline \multicolumn{1}{|c|}{ Parameter } & \multicolumn{1}{c|}{ Value } \\
1 Title & zgy-4-113-CC \\
2 Origin & Bruker BioSpin GmbH \\
3 Solvent & CDC13 \\
4 Temperature & 300.0 \\
5 Number of Scans & 93 \\
6 Acquisition Time & 1.3631 \\
7 Acquisition Date & $2020-01-04 T 09: 41: 05$ \\
8 Spectrometer Frequency 100.61 \\
9 Spectral Width & 24038.5 \\
\hline
\end{tabular}
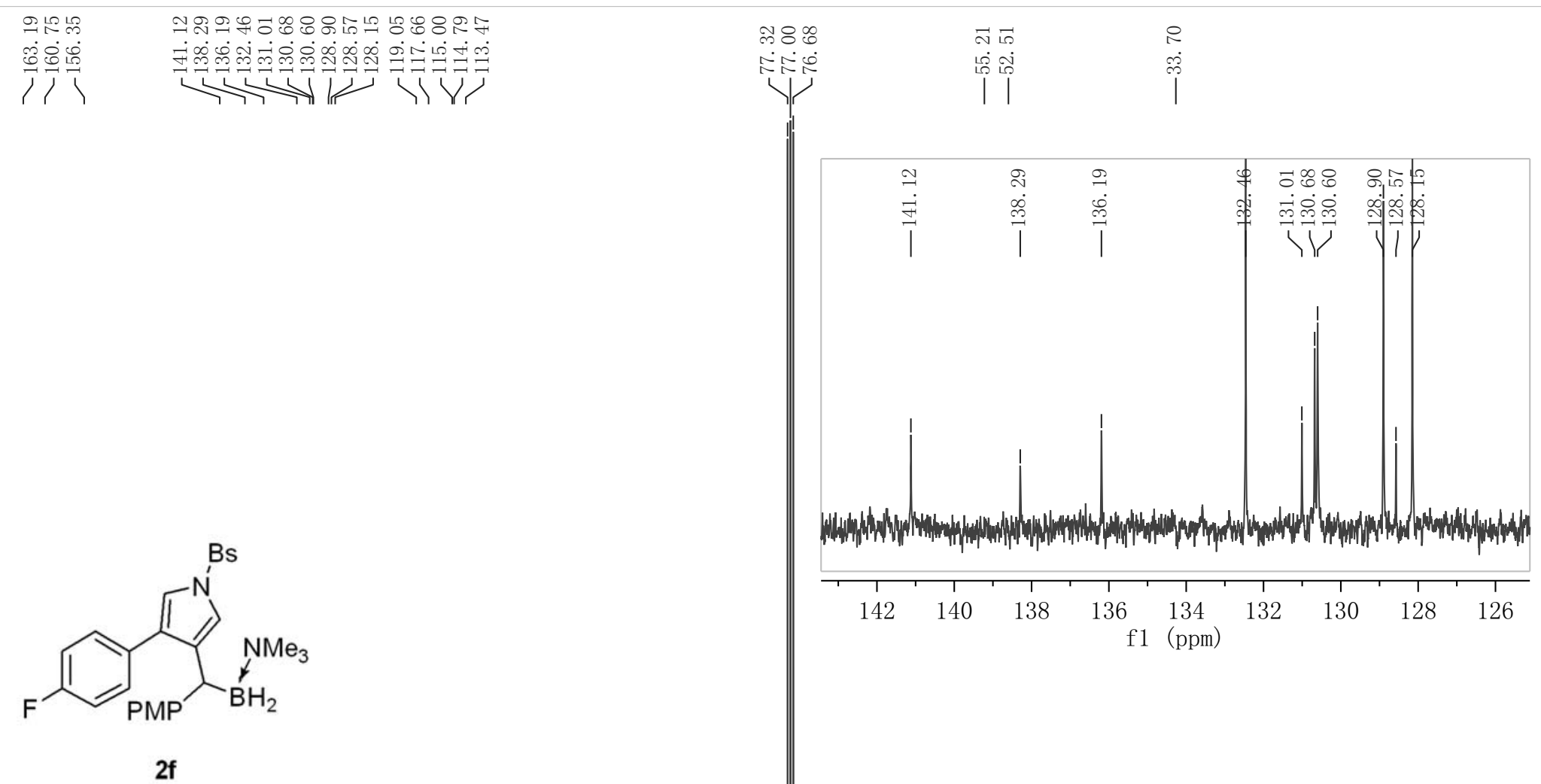

f1 (ppm)

$2 f$

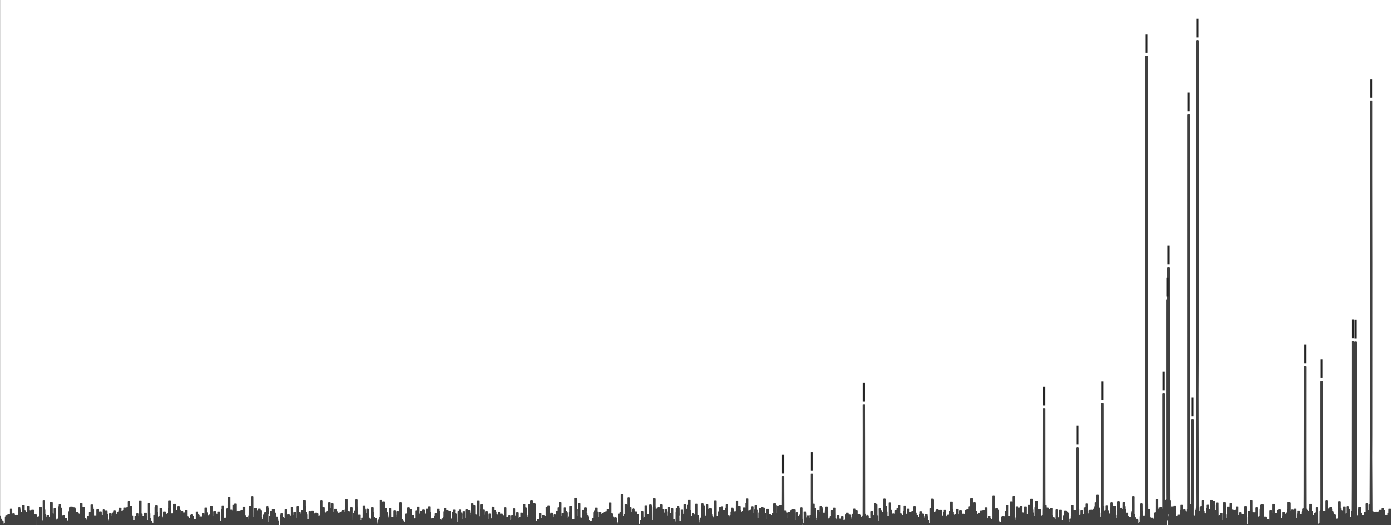

M.t.

1.m 


\begin{tabular}{|ll|}
\hline \multicolumn{1}{|c|}{ Parameter } & \multicolumn{1}{c|}{ Value } \\
1 Title & zgy-4-113-F \\
2 Origin & Bruker BioSpin GmbH \\
3 Solvent & CDC13 \\
4 Temperature & 297.4 \\
5 Number of Scans & 16 \\
6 Acquisition Time & 0.7340 \\
7 Acquisition Date & $2021-08-27 T 10: 33: 54$ \\
8 Spectrometer Frequency & 376.31 \\
9 Spectral Width & 89285.7 \\
\hline
\end{tabular}

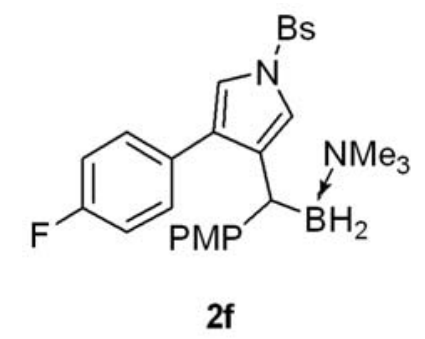

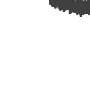

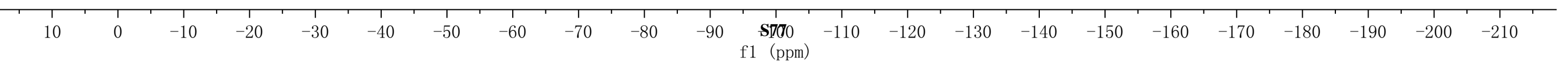




\begin{tabular}{|c|c|}
\hline Parameter & Value \\
\hline $\begin{array}{l}1 \text { Title } \\
2 \text { Origin }\end{array}$ & zgy $-4-117-\mathrm{H}$ \\
\hline 3 Solvent & $\mathrm{CDC} \_3$ \\
\hline 4 Temperature & 298.9 \\
\hline 5 Number of Scans & 16 \\
\hline 6 Acquisition Time & 4. 0002 \\
\hline 7 Acquisition Date & $2021-08-17 \mathrm{~T} 23: 34: 29$ \\
\hline 8 Spectrometer Frequency & 399.93 \\
\hline 9 Spectral Width & 8012.0 \\
\hline
\end{tabular}

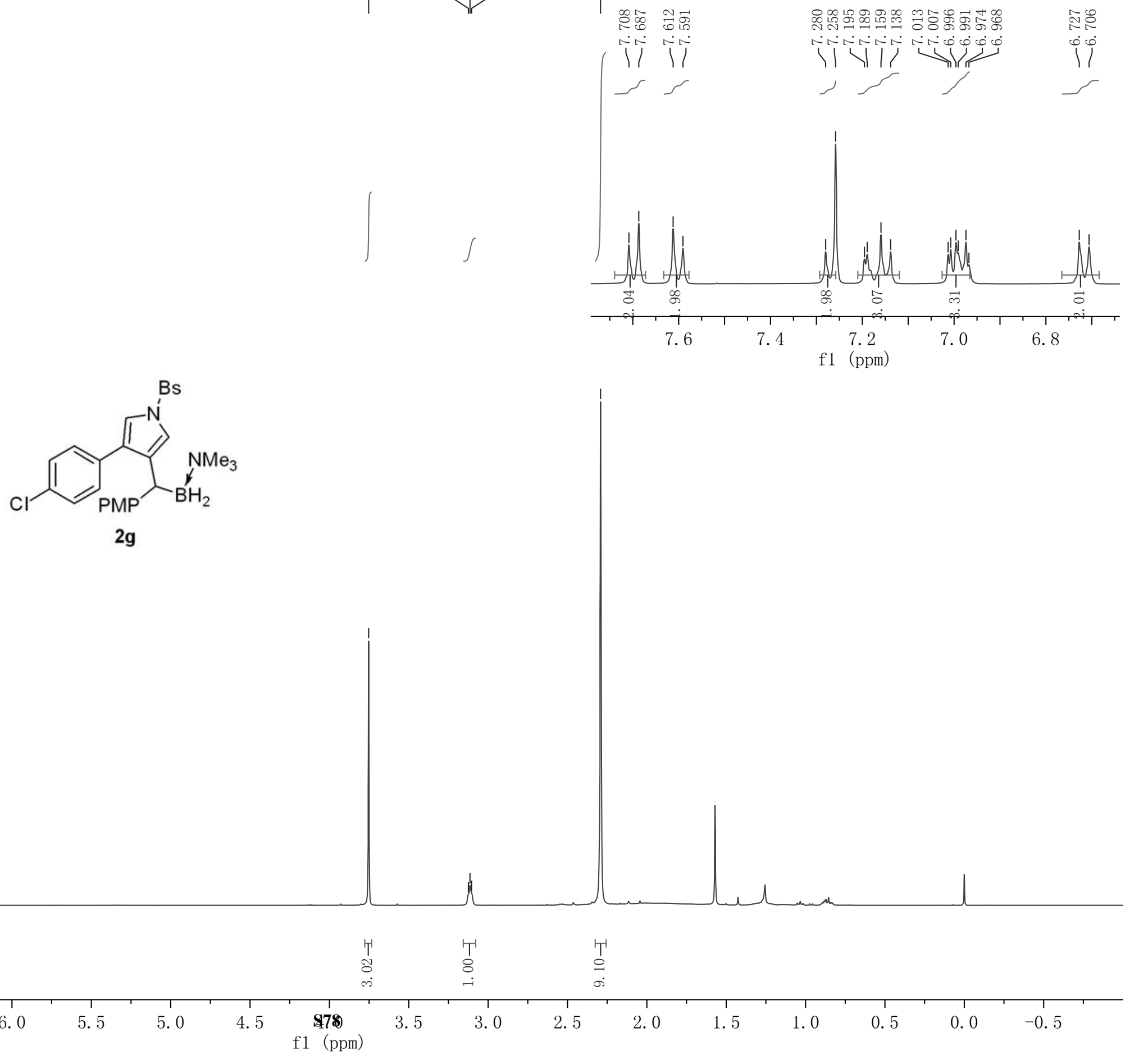




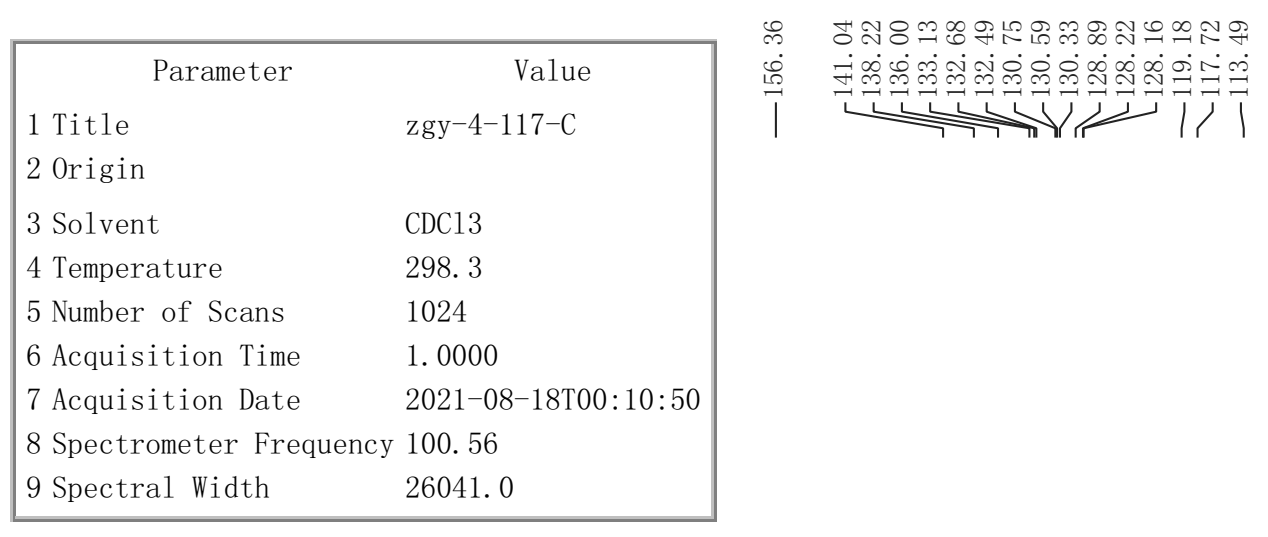

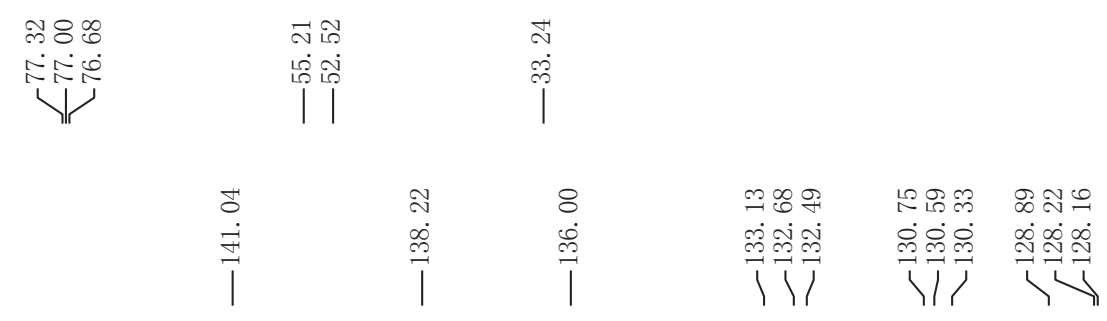
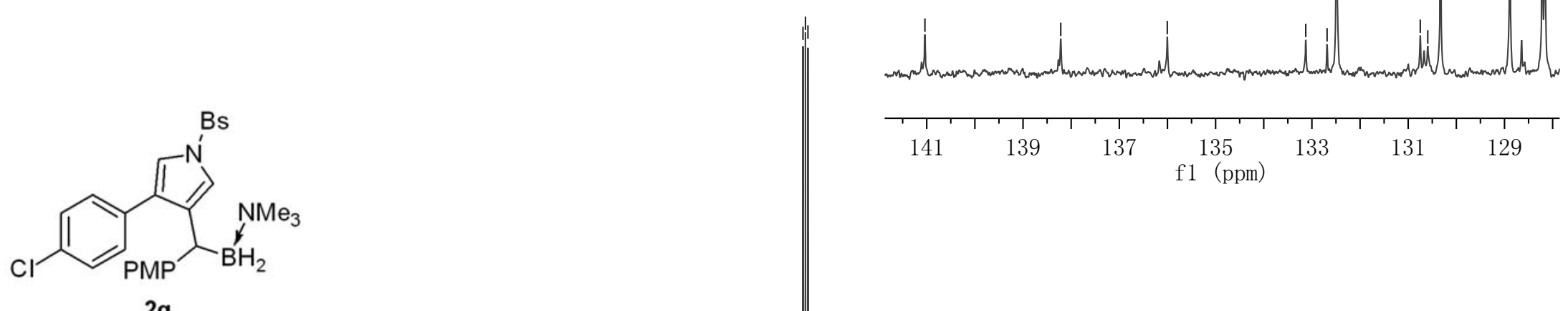

2g

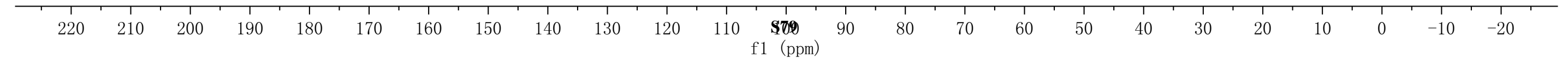




\begin{tabular}{|ll|}
\hline \multicolumn{1}{|c|}{ Parameter } & \multicolumn{1}{c|}{ Value } \\
1 Title & zgy $-4-118-\mathrm{C}$ \\
2 Origin & \\
3 Solvent & $\mathrm{CDCl3}$ \\
4 Temperature & 299.3 \\
5 Number of Scans & 1024 \\
6 Acquisition Time & 1.0000 \\
7 Acquisition Date & $2021-08-16 \mathrm{~T} 23: 10: 12$ \\
8 Spectrometer Frequency 100.56 \\
9 Spectral Width & 26041.0 \\
\hline
\end{tabular}

ヘ

ம.

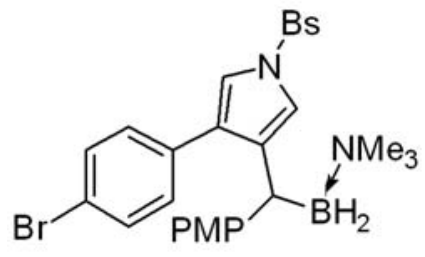

2h

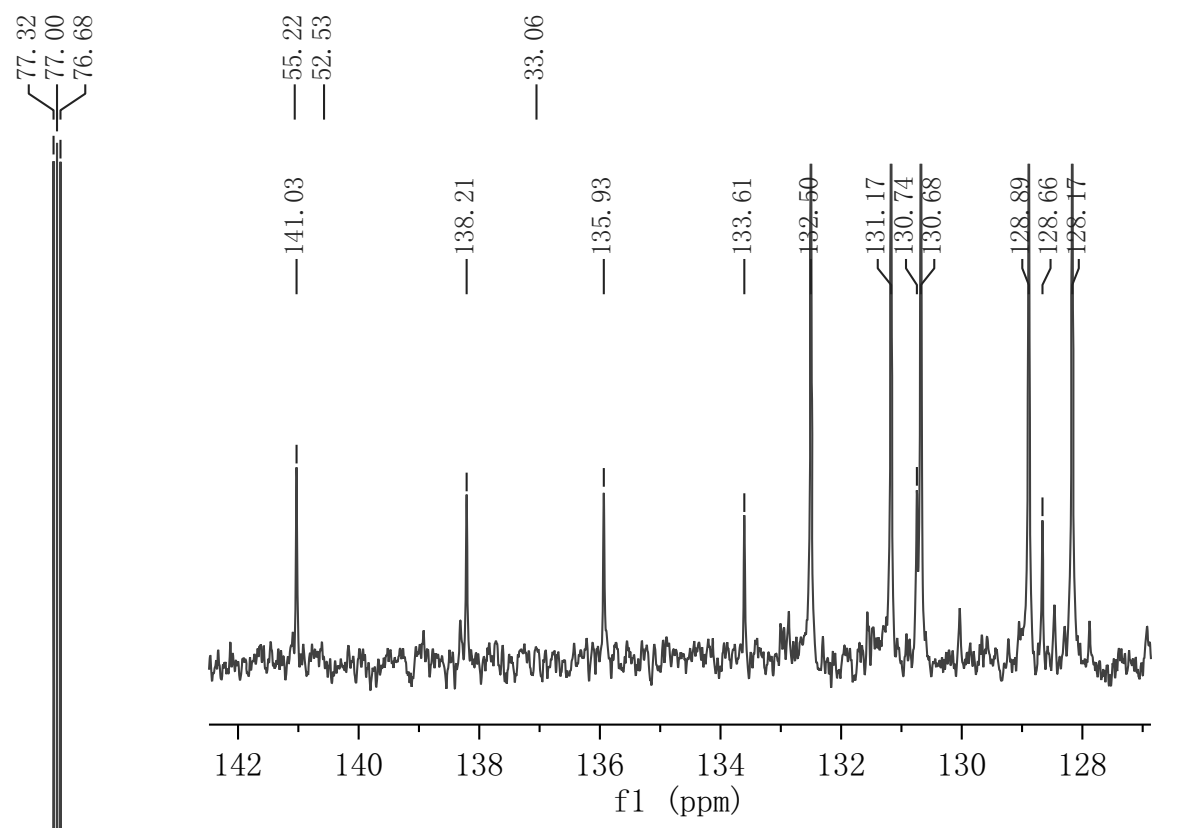

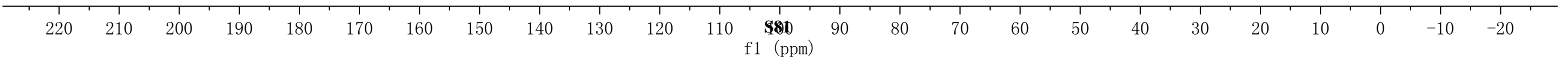




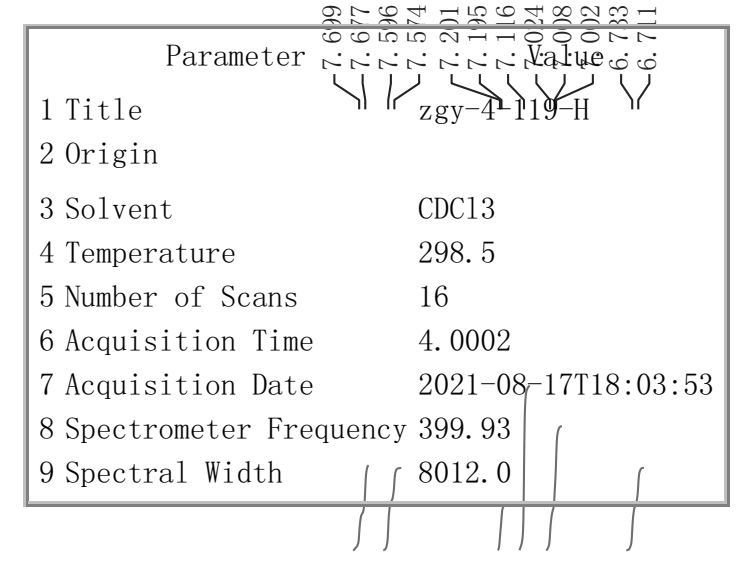

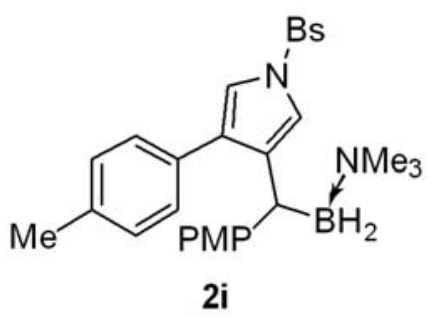

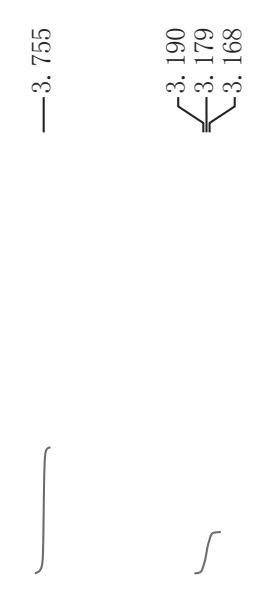

윙

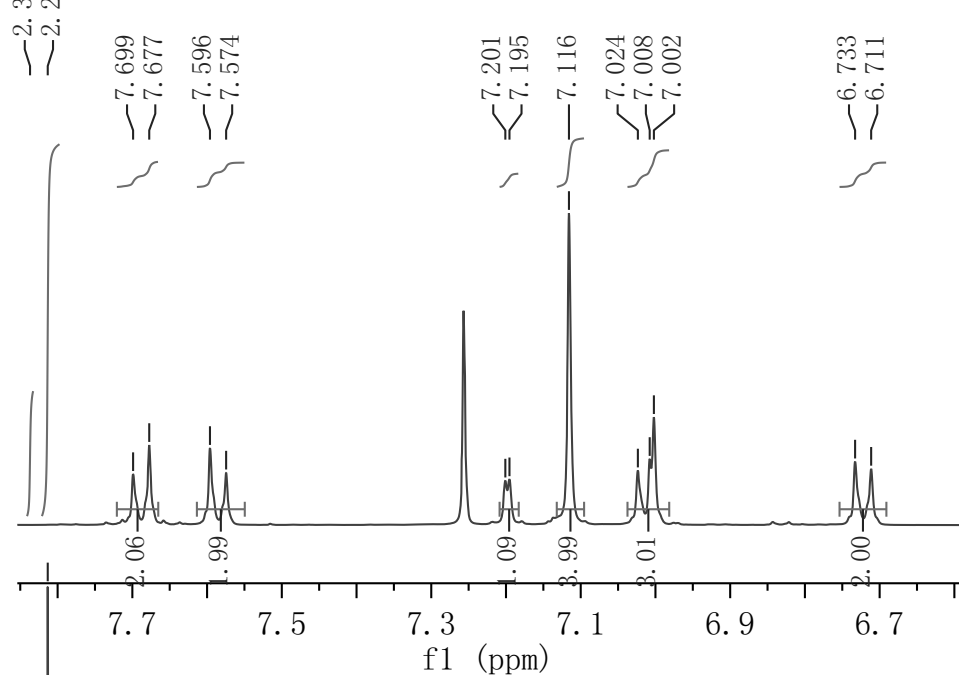

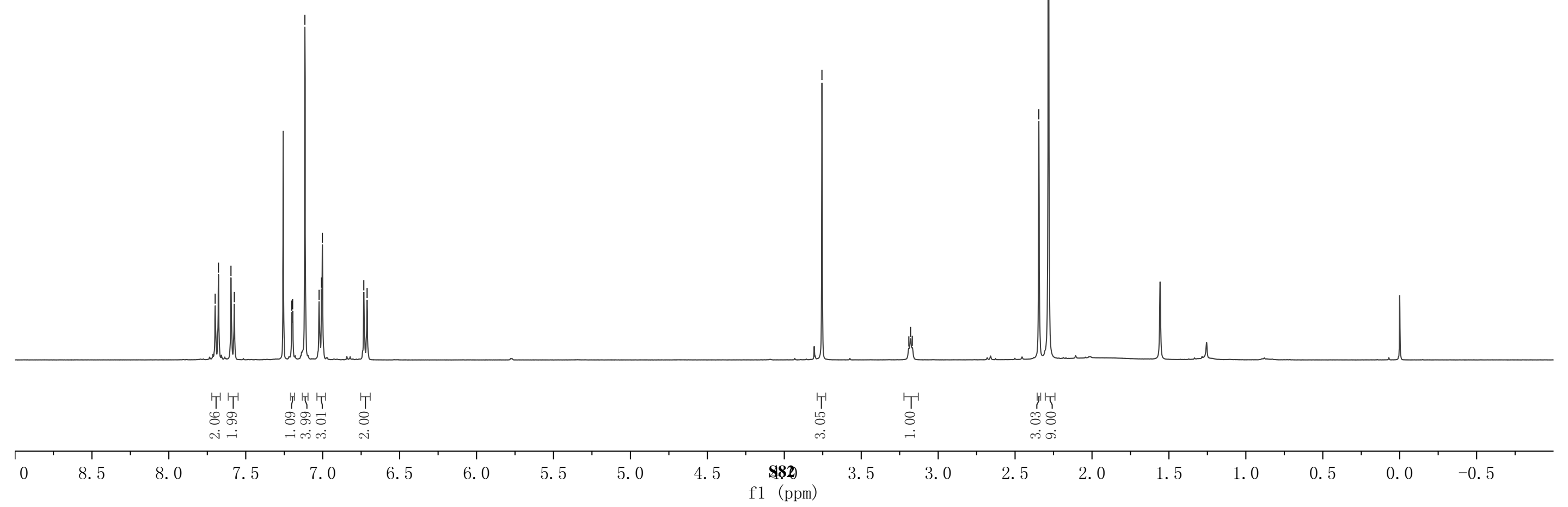




\begin{tabular}{|ll|}
\hline \multicolumn{1}{|c|}{ Parameter } & \multicolumn{1}{c|}{ Value } \\
1 Title & zgy $-4-119-\mathrm{C}$ \\
2 Origin & $\mathrm{CDCl3}$ \\
3 Solvent & 298.6 \\
4 Temperature & 500 \\
5 Number of Scans & 1.0000 \\
6 Acquisition Time & $2021-08-17 \mathrm{~T} 18: 22: 41$ \\
7 Acquisition Date & 100.56 \\
8 Spectrometer Frequency & 26041.0 \\
9 Spectral Width & \\
\hline
\end{tabular}
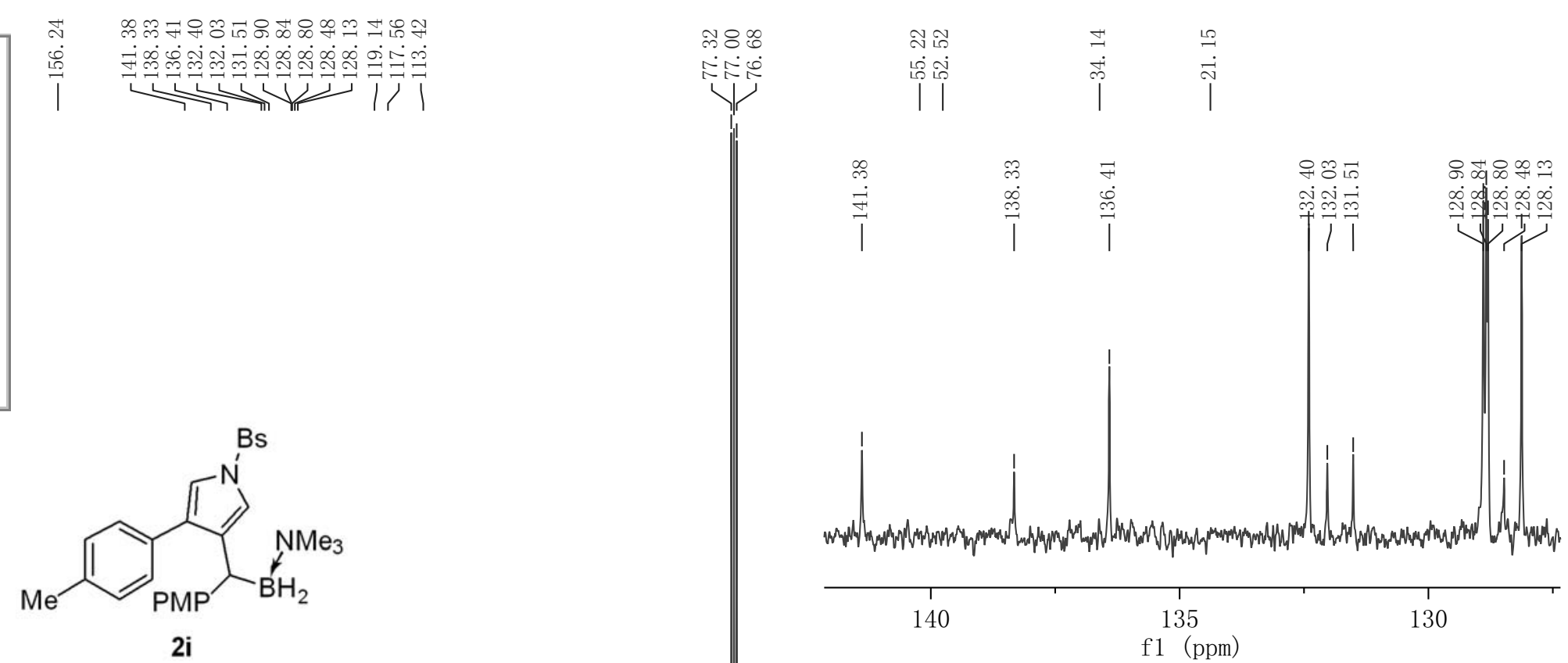

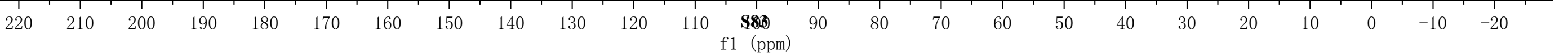




\begin{tabular}{|c|c|}
\hline Parameter & Value \\
\hline 1 Title & zgy-4-108-H \\
\hline 2 Origin & Bruker BioSpin GmbH \\
\hline 3 Solvent & $\mathrm{CDCl3}$ \\
\hline 4 Temperature & 298.0 \\
\hline 5 Number of Scans & 9 \\
\hline 6 Acquisition Time & 4. 0894 \\
\hline 7 Acquisition Date & $2019-12-03 T 16: 59: 52$ \\
\hline \multicolumn{2}{|c|}{8 Spectrometer Frequency 400. 13} \\
\hline 9 Spectral Width & 8012.8 \\
\hline
\end{tabular}
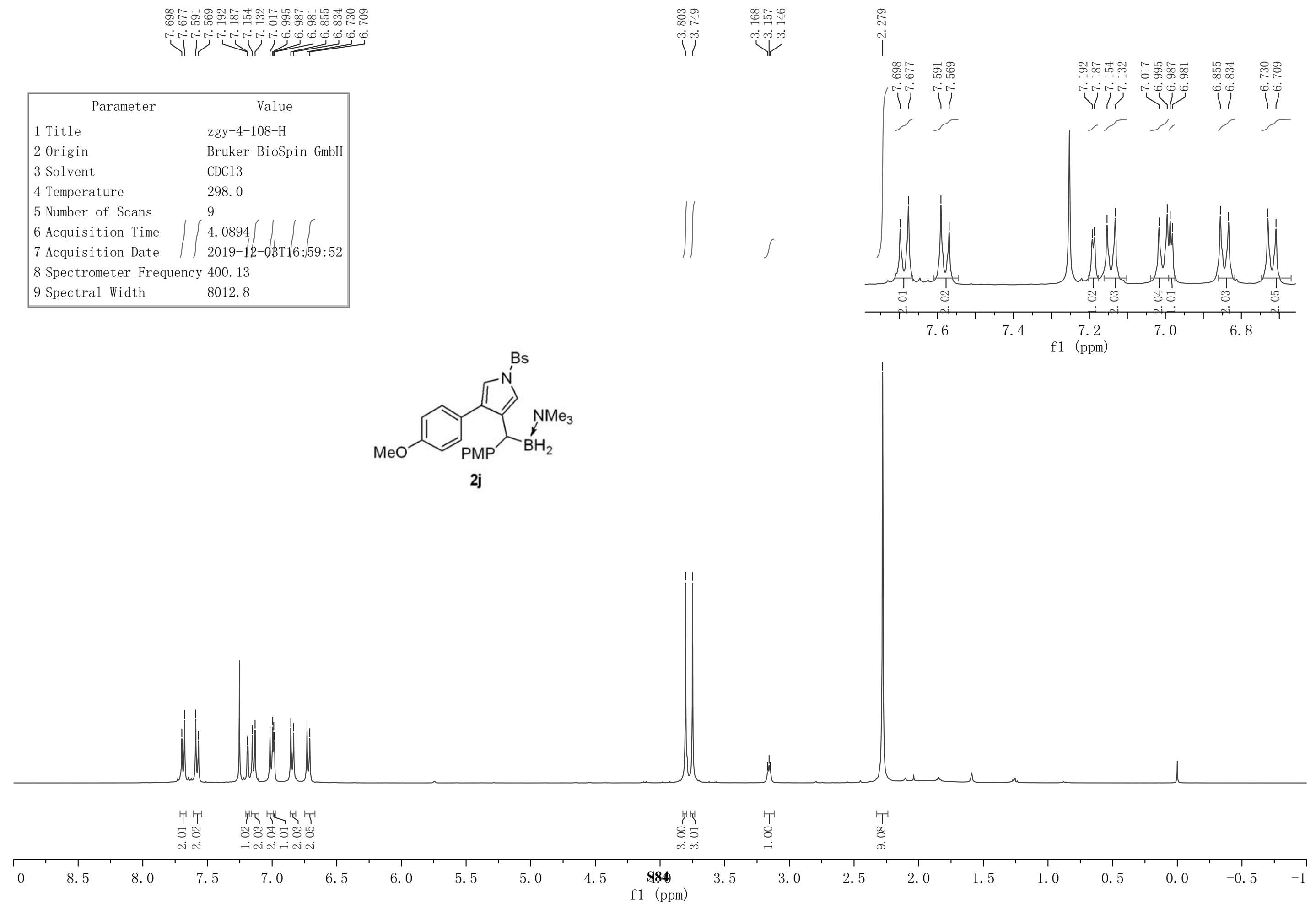


\begin{tabular}{|ll|}
\hline \multicolumn{1}{|c|}{ Parameter } & \multicolumn{1}{c|}{ Value } \\
1 Title & zgy-4-108-C \\
2 Origin & Bruker BioSpin GmbH \\
3 Solvent & CDC13 \\
4 Temperature & 300.0 \\
5 Number of Scans & 61 \\
6 Acquisition Time & 1.3631 \\
7 Acquisition Date & $2019-12-03 \mathrm{~T} 17: 02: 25$ \\
8 Spectrometer Frequency 100.61 \\
9 Spectral Width & 24038.5 \\
\hline
\end{tabular}

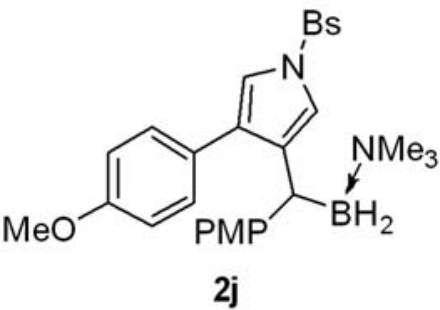

\section{Ant}

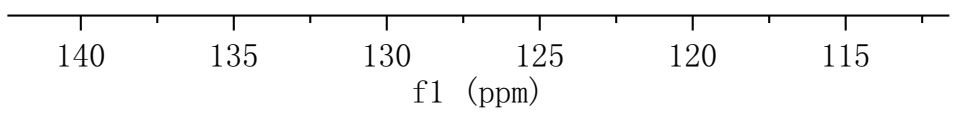

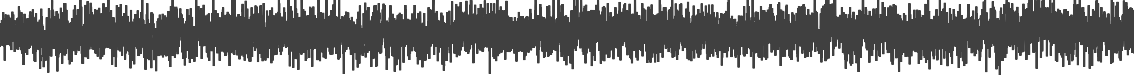
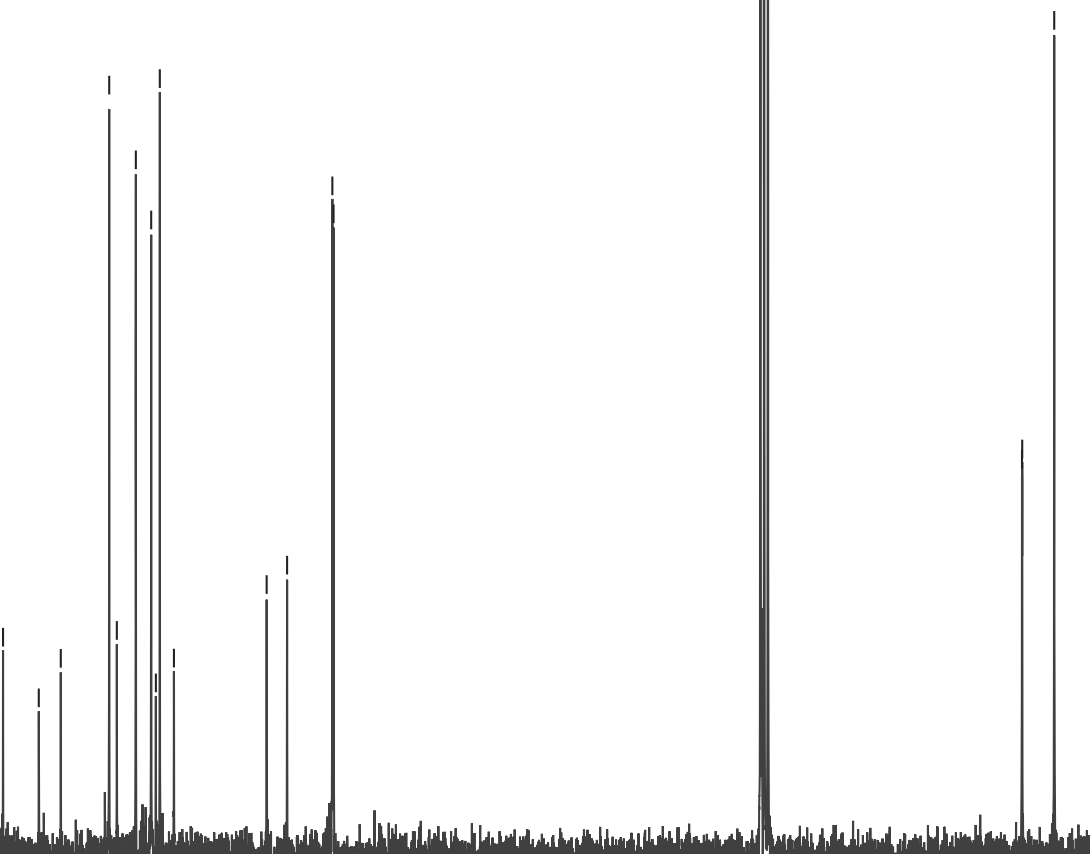

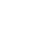




\begin{tabular}{|ll|}
\hline \multicolumn{1}{|c|}{ Parameter } & \multicolumn{1}{c|}{ Value } \\
1 Title & zgy-4-146-H \\
2 Origin & Bruker BioSpin GmbH \\
3 Solvent & CDC13 \\
4 Temperature & 298.0 \\
5 Number of Scans & 15 \\
6 Acquisition Time & 4.0894 \\
7 Acquisition Date \\
8 Spectrometer Frequency 400.13 \\
9 Spectral Width & 8012.8 \\
\hline
\end{tabular}
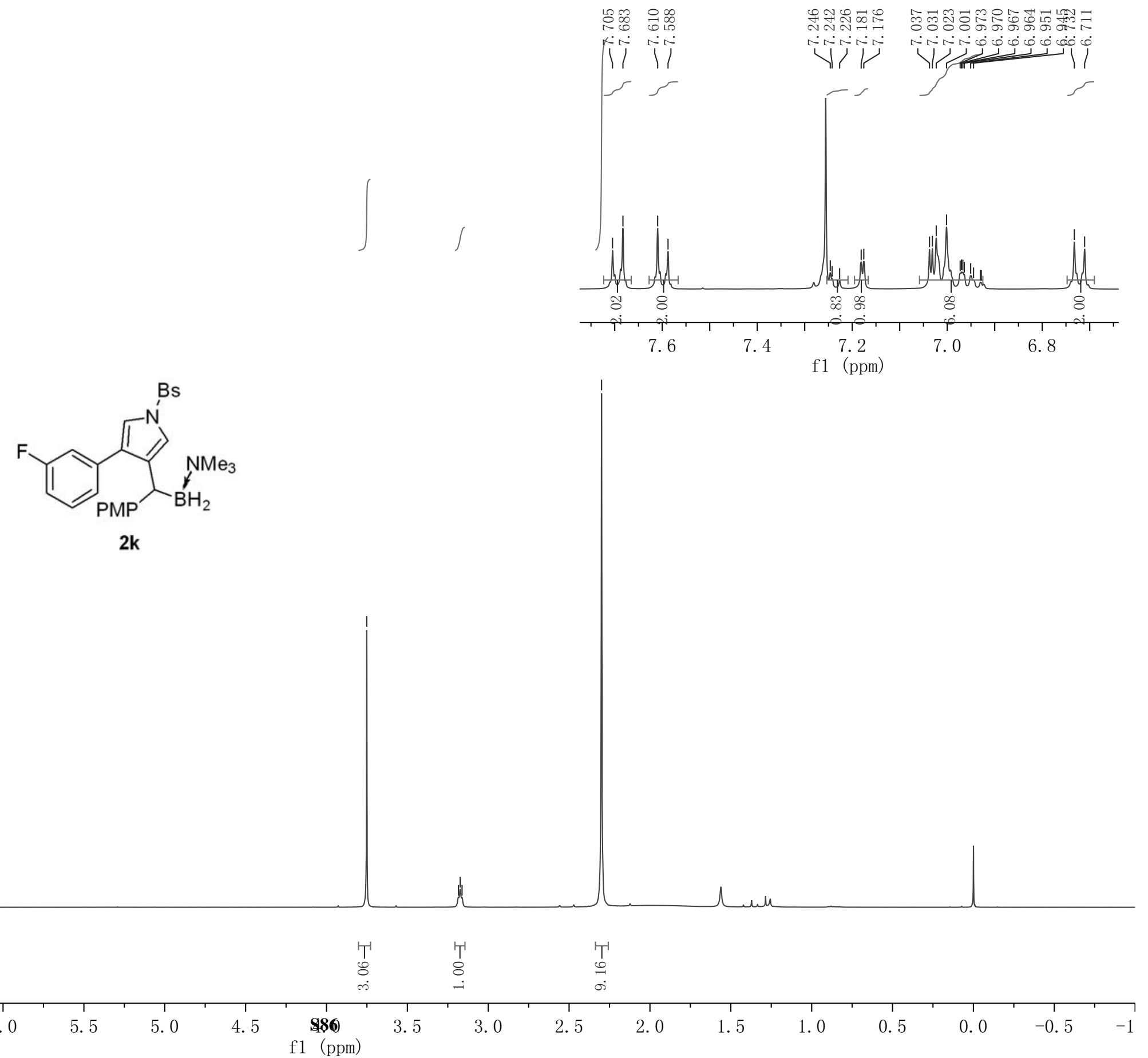

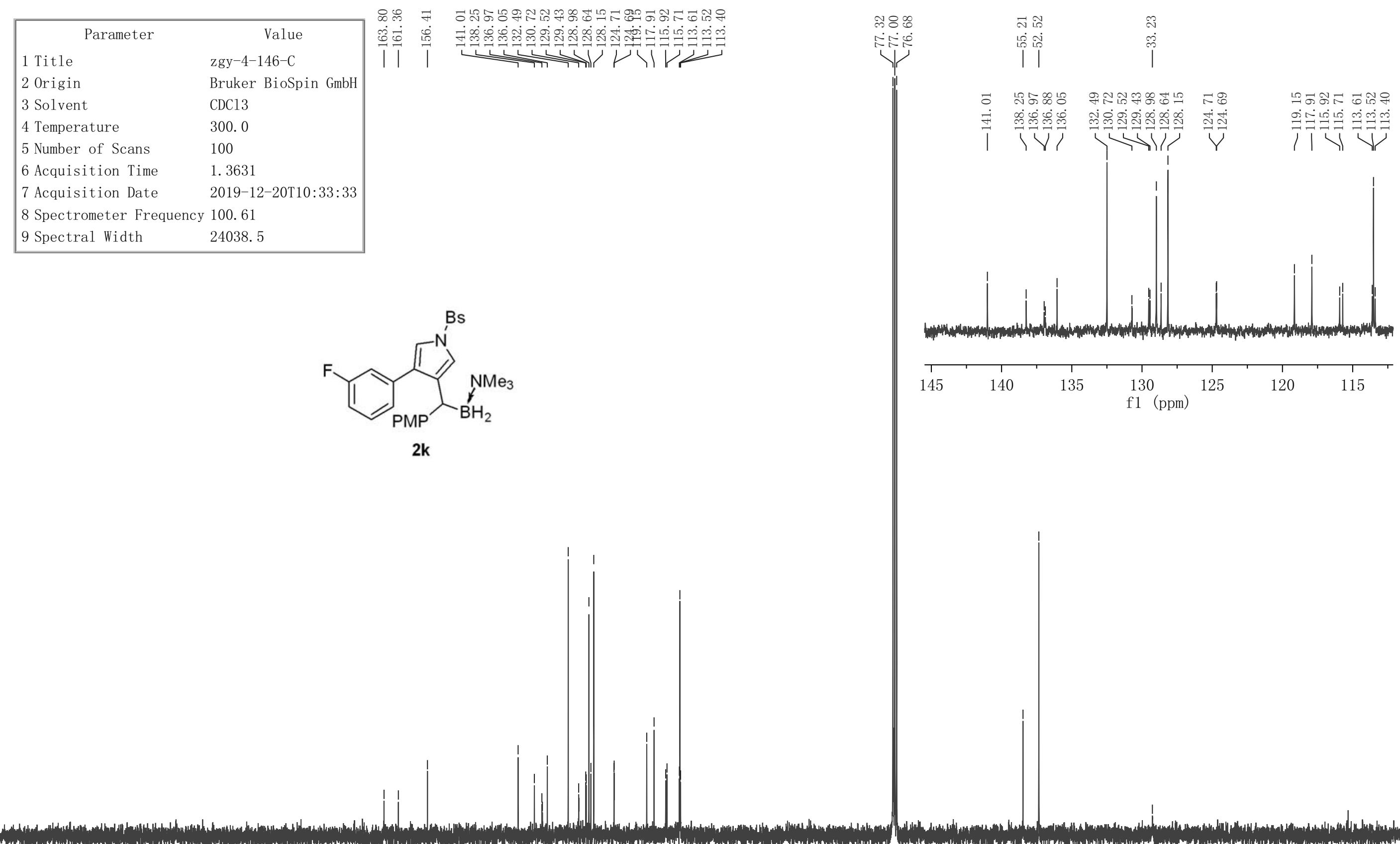

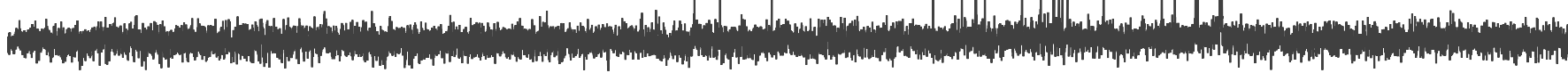

\begin{tabular}{|c|c|c|c|c|c|c|c|c|c|c|c|c|c|c|c|c|c|c|c|c|}
\hline & 210 & 200 & 190 & $\begin{array}{c}1 \\
180\end{array}$ & 170 & 160 & 150 & 140 & 13 & 120 & $\begin{array}{c}\mathbf{5 8 7} \\
1 \quad(\mathrm{ppm})\end{array}$ & 100 & 90 & 80 & 70 & 60 & 50 & 40 & 3 & 20 \\
\hline
\end{tabular}




\begin{tabular}{|ll|}
\hline \multicolumn{1}{|c|}{ Parameter } & \multicolumn{1}{c|}{ Value } \\
1 Title & zgy-4-146-F \\
2 Origin & Bruker BioSpin GmbH \\
3 Solvent & CDC13 \\
4 Temperature & 297.1 \\
5 Number of Scans & 16 \\
6 Acquisition Time & 0.7340 \\
7 Acquisition Date & $2021-08-27 T 18: 14: 49$ \\
8 Spectrometer Frequency & 376.31 \\
9 Spectral Width & 89285.7 \\
\hline
\end{tabular}

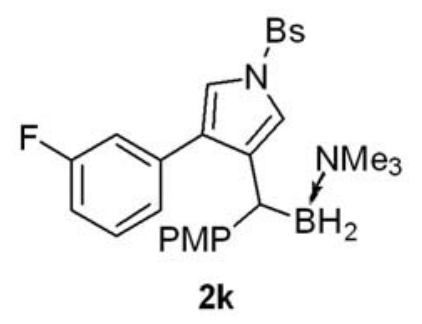

\begin{tabular}{|c|c|c|c|c|c|c|c|c|c|c|c|c|c|c|c|c|c|c|c|c|c|}
\hline 10 & 0 & -10 & -20 & -30 & -40 & -50 & -60 & -70 & -80 & -90 & $\begin{array}{c}\mathbf{S 8 8 0} \\
\mathrm{f1} \quad \stackrel{(\mathrm{ppm})}{ }\end{array}$ & -110 & -120 & -130 & -140 & -150 & -160 & -170 & -180 & -190 & $\begin{array}{ll}-200 & -210\end{array}$ \\
\hline
\end{tabular}




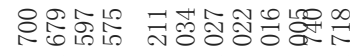

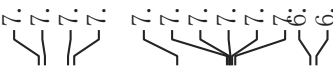

\begin{tabular}{|c|c|}
\hline Parameter & Value \\
\hline 1 Title & $\mathrm{zgy}-4-135-\mathrm{H}$ \\
\hline 2 Origin & Bruker BioSpin GmbH \\
\hline 3 Solvent & $\mathrm{CDC13}$ \\
\hline 4 Temperature & 298.0 \\
\hline 5 Number of Scans & $8 /$ \\
\hline 6 Acquisition Time & 4. 0894 \\
\hline 7 Acquisition Date & $2019-12-14 \mathrm{~T} 21: 38: 59$ \\
\hline 8 Spectrometer Frequency & 400.13 \\
\hline 9 Spectral Width & 8012.8 \\
\hline
\end{tabular}

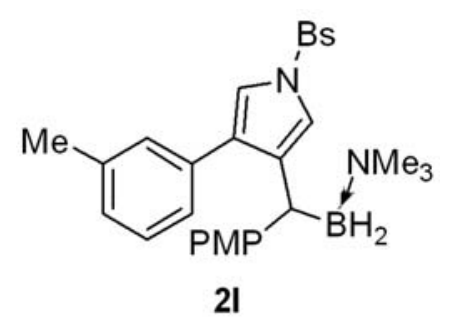

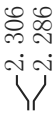

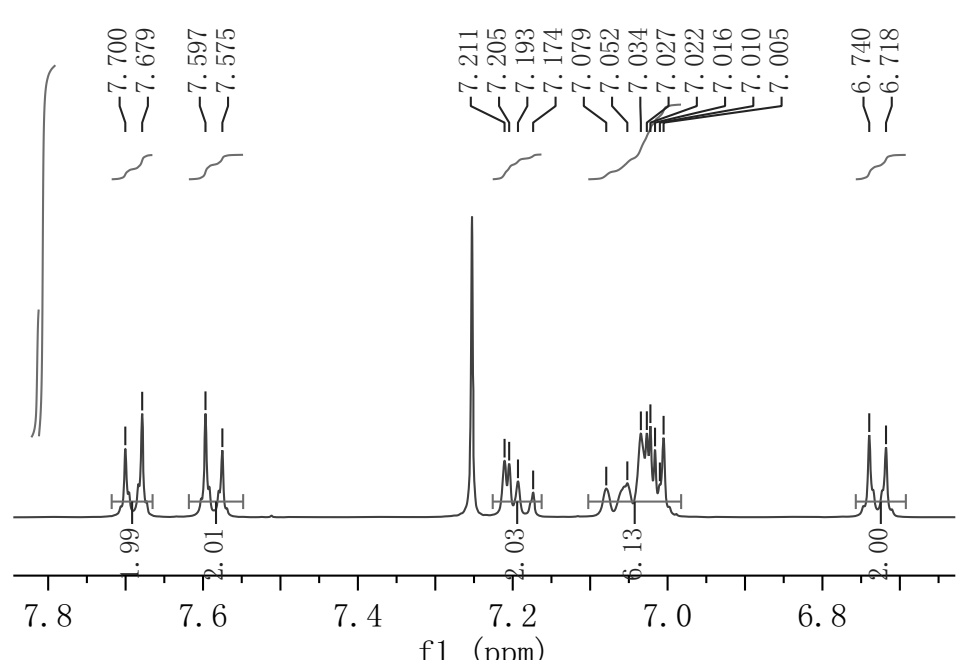

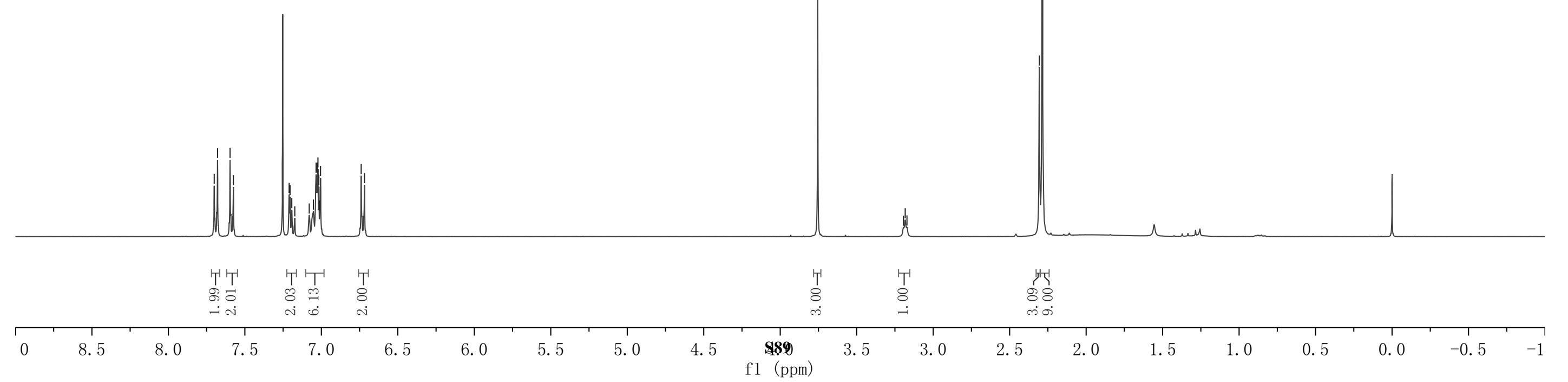




\begin{tabular}{|ll|}
\hline \multicolumn{1}{|c|}{ Parameter } & \multicolumn{1}{c|}{ Value } \\
1 Title & zgy-4-135-C \\
2 Origin & Bruker BioSpin GmbH \\
3 Solvent & CDC13 \\
4 Temperature & 300.0 \\
5 Number of Scans & 55 \\
6 Acquisition Time & 1.3631 \\
7 Acquisition Date & $2019-12-14 \mathrm{~T} 21: 41: 33$ \\
8 Spectrometer Frequency 100.61 \\
9 Spectral Width & 24038.5 \\
\hline
\end{tabular}
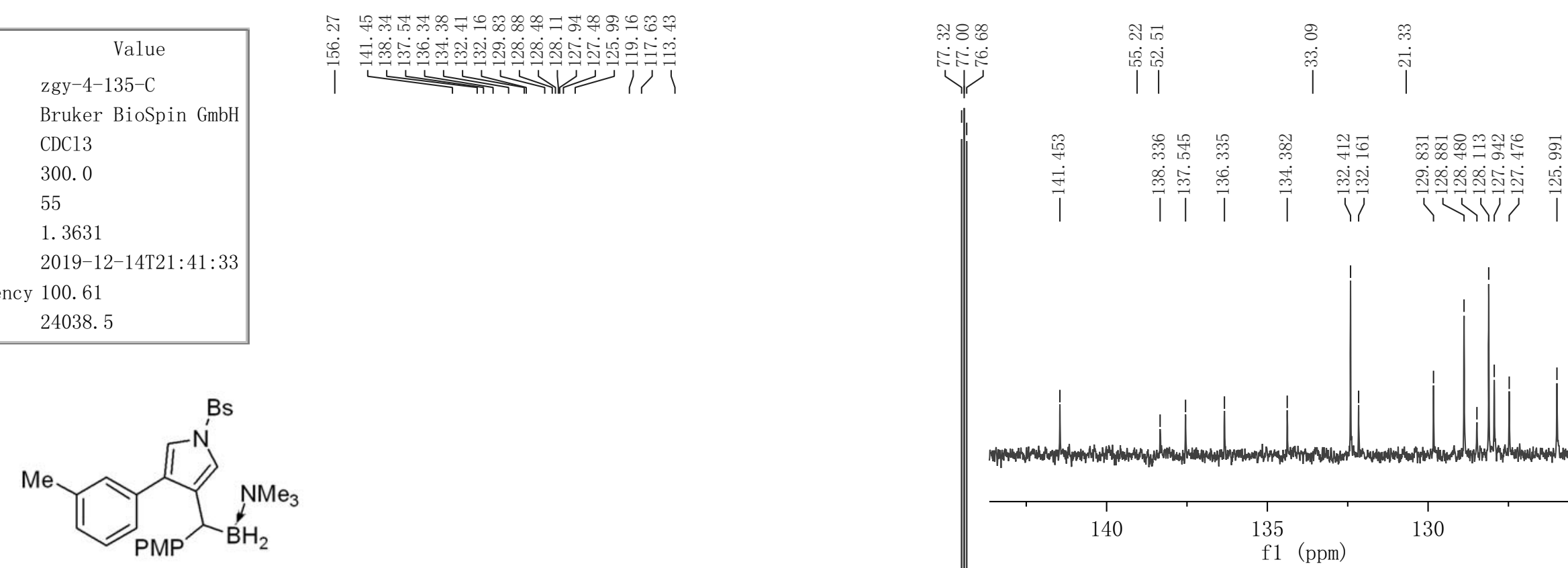

2I
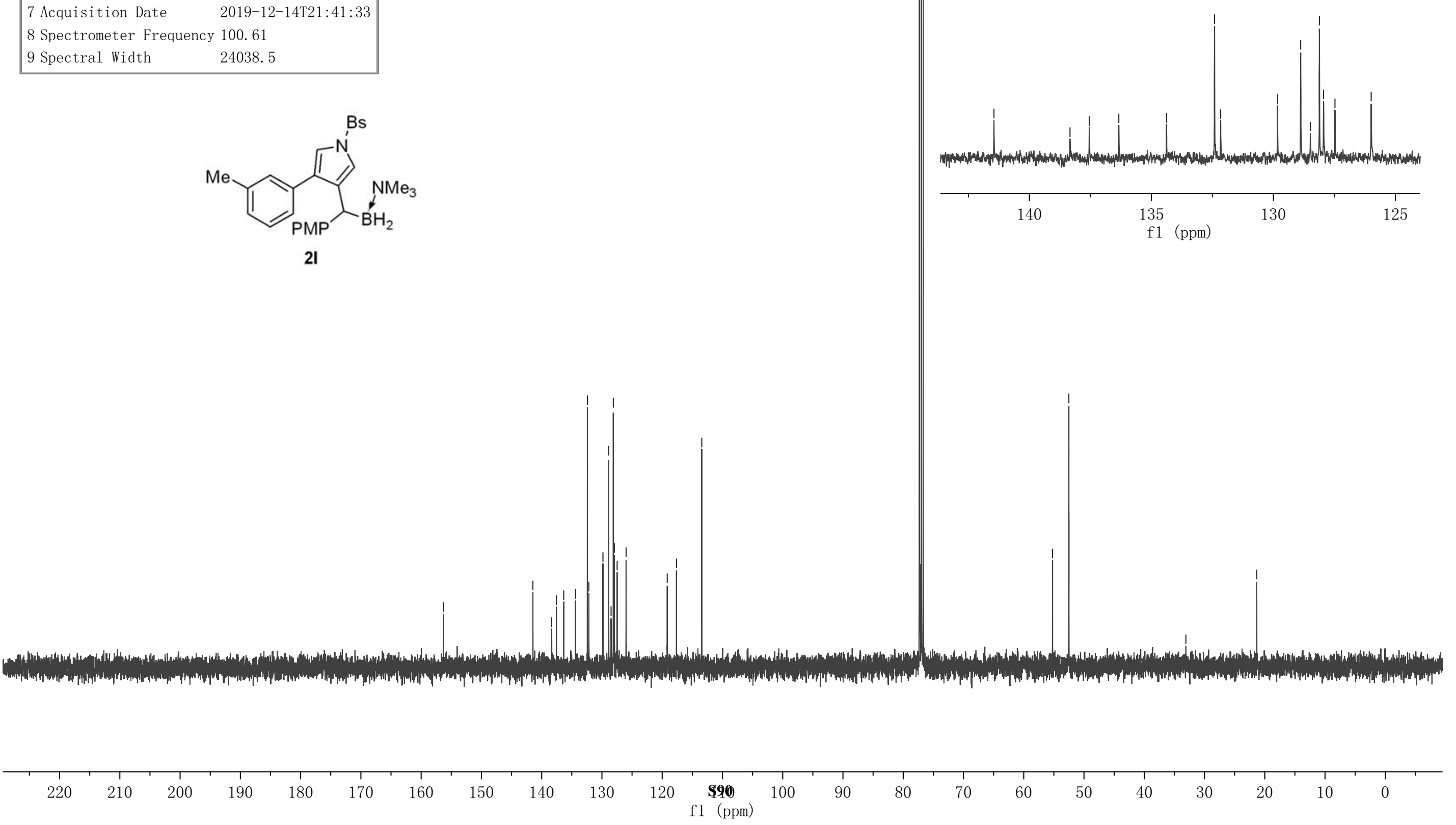


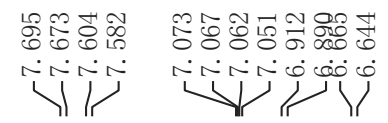

\begin{tabular}{|ll|}
\hline \multicolumn{1}{|c|}{ Parameter } & \multicolumn{1}{c|}{ Value } \\
1 Title & ZGY-4-143-hh \\
2 Origin & Bruker BioSpin GmbH \\
3 Solvent & CDC13 \\
4 Temperature & $298 . \varnothing$ \\
5 Number of Scans & 7 \\
6 Acquisition Time & 4.0894 \\
7 Acquisition Date & $2 / 019-12-18 \mathrm{~T} / 16: 57: 20$ \\
8 Spectrometer Frequency & 400.13 \\
9 Spectral Width & 8012.8 \\
\hline
\end{tabular}

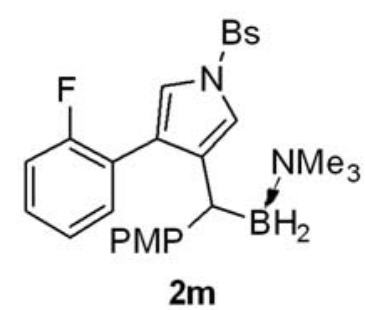

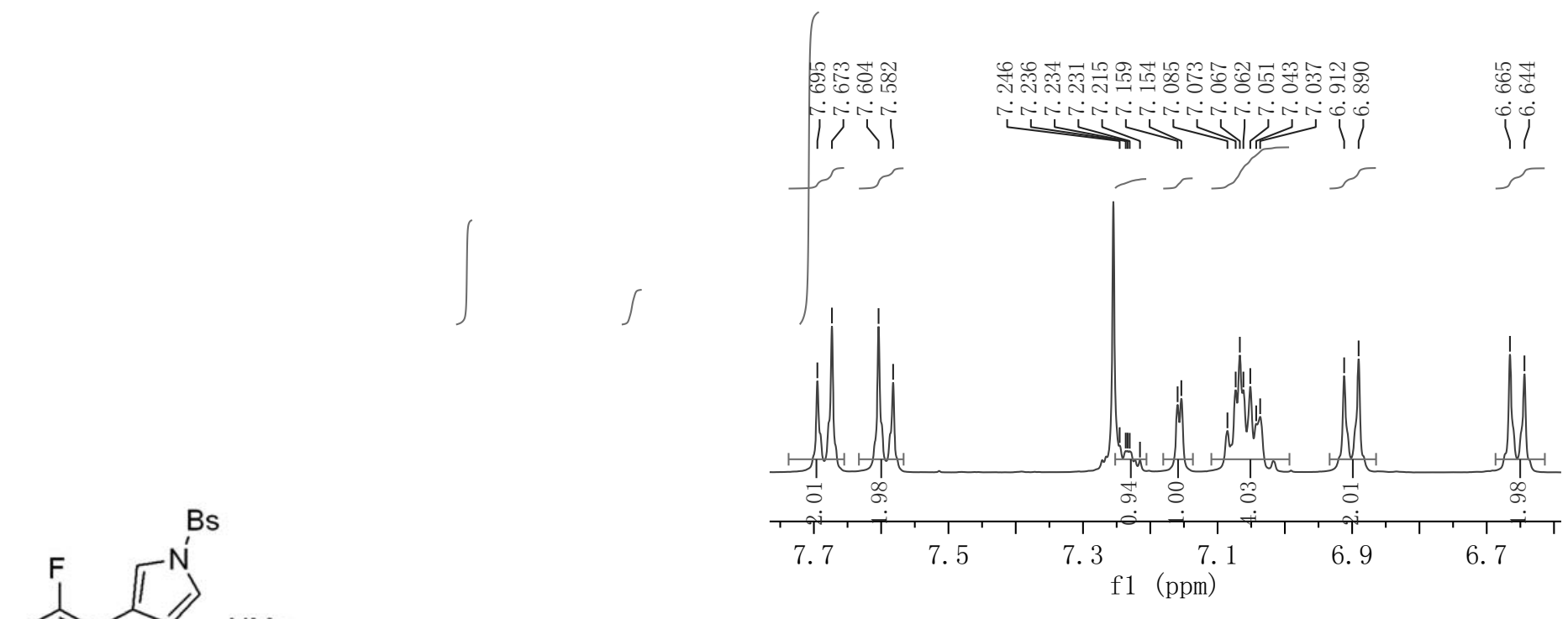

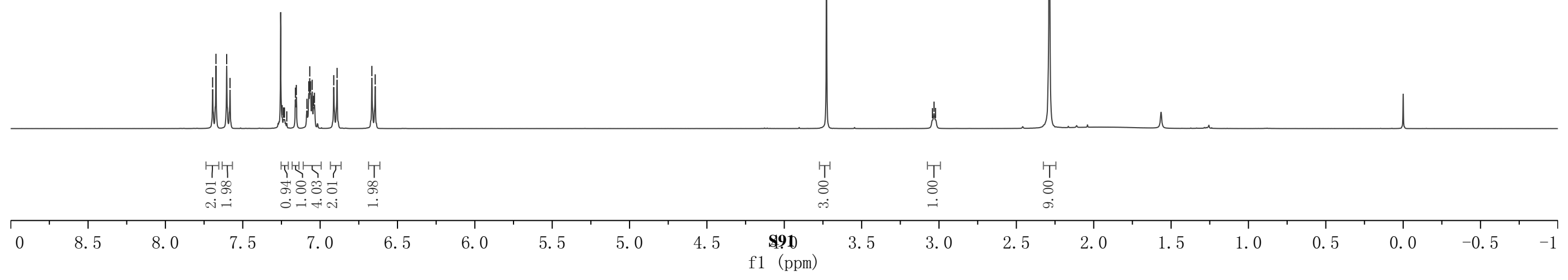




\begin{tabular}{|ll|}
\hline \multicolumn{1}{|c|}{ Parameter } & \multicolumn{1}{c|}{ Value } \\
1 Title & zgy-4-143-CCC \\
2 Origin & Bruker BioSpin GmbH \\
3 Solvent & CDC13 \\
4 Temperature & 300.0 \\
5 Number of Scans & 140 \\
6 Acquisition Time & 1.3631 \\
7 Acquisition Date & $2019-12-19 \mathrm{~T} 10: 25: 36$ \\
8 Spectrometer Frequency & 100.61 \\
9 Spectral Width & 24038.5 \\
\hline
\end{tabular}

$111 \%$

$\mid$
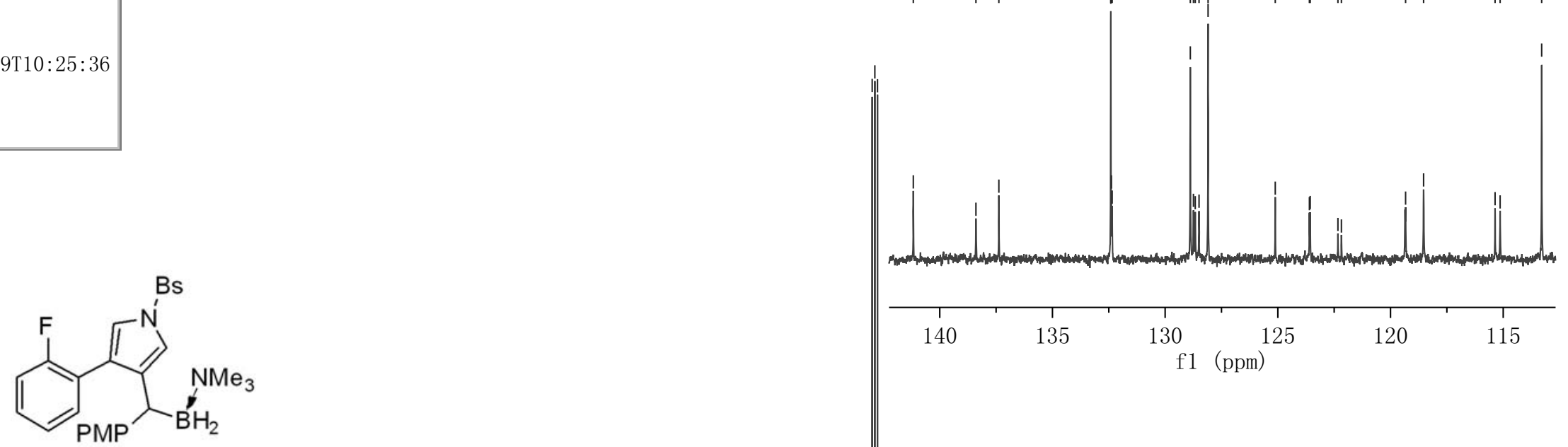

$2 m$

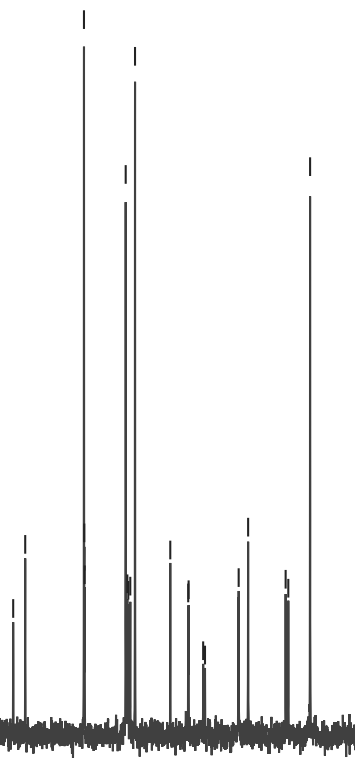




\begin{tabular}{|ll|}
\hline \multicolumn{1}{|c|}{ Parameter } & \multicolumn{1}{c|}{ Value } \\
1 Title & ZGY-4-143-F \\
2 Origin & Bruker BioSpin GmbH \\
3 Solvent & CDC13 \\
4 Temperature & 297.3 \\
5 Number of Scans & 16 \\
6 Acquisition Time & 0.7340 \\
7 Acquisition Date & $2021-08-27$ T10:26:59 \\
8 Spectrometer Frequency 376.31 \\
9 Spectral Width & 89285.7 \\
\hline
\end{tabular}

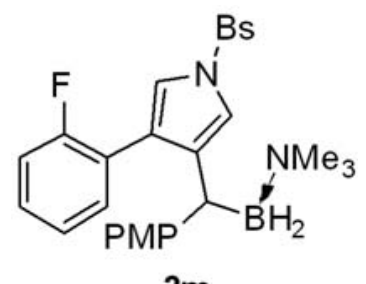

$2 \mathrm{~m}$ 


\begin{tabular}{|c|c|}
\hline Parameter & Value \\
\hline 1 Title & ZGY-4-136-H \\
\hline 2 Origin & Bruker BioSpin GmbH \\
\hline 3 Solvent & $\mathrm{CDC} 13$ \\
\hline 4 Temperature & 298.0 \\
\hline 5 Number of Scans & 6 \\
\hline 6 Acquisition Time & 4. 0894 \\
\hline 7 Acquisition Date & 2019-12ך16 17 \\
\hline \multicolumn{2}{|c|}{8 Spectrometer Frequency 400.13 J/ J } \\
\hline 9 Spectral Width & 8012.8 \\
\hline
\end{tabular}
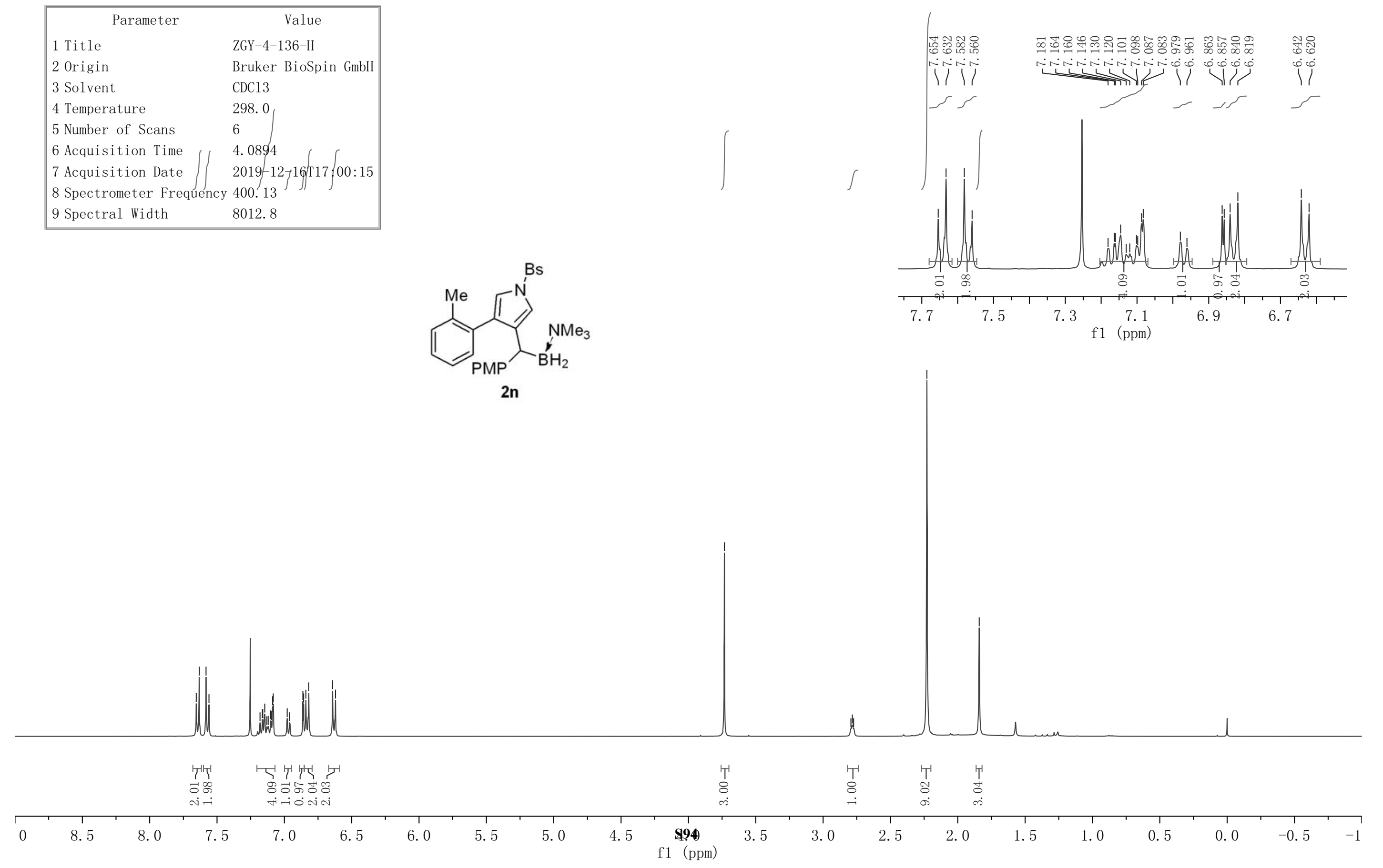


\begin{tabular}{|ll|}
\hline \multicolumn{1}{|c|}{ Parameter } & \multicolumn{1}{c|}{ Value } \\
1 Title & ZGY-4-136-C \\
2 Origin & Bruker BioSpin GmbH \\
3 Solvent & CDC13 \\
4 Temperature & 300.0 \\
5 Number of Scans & 42 \\
6 Acquisition Time & 1.3631 \\
7 Acquisition Date & $2019-12-16 \mathrm{~T} 17: 02: 48$ \\
8 Spectrometer Frequency 100.61 \\
9 Spectral Width & 24038.5 \\
\hline
\end{tabular}
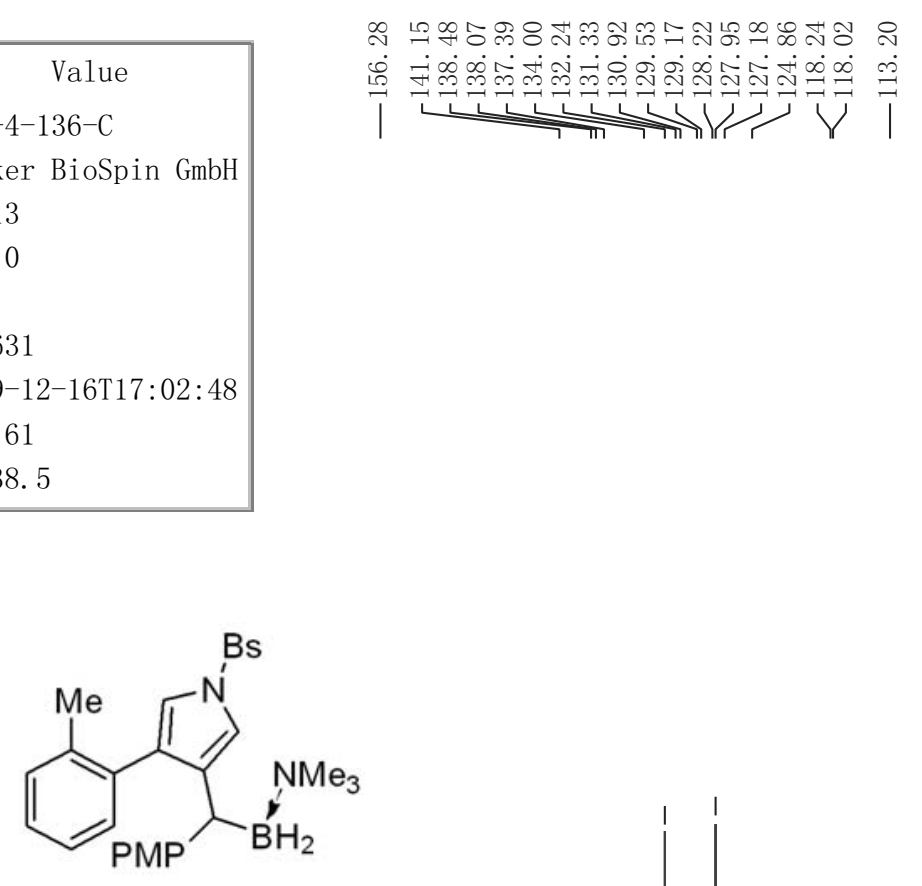

$2 n$

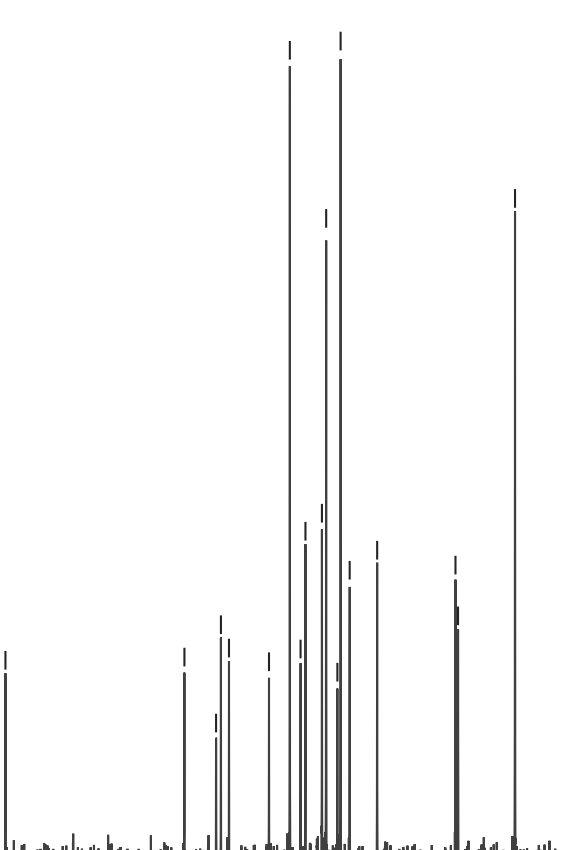

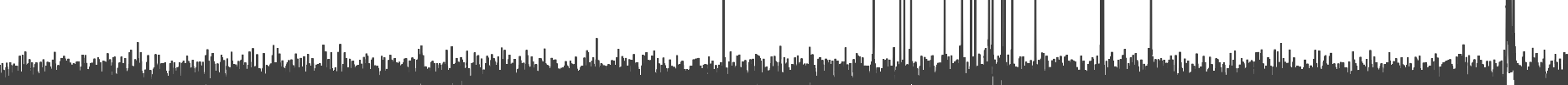
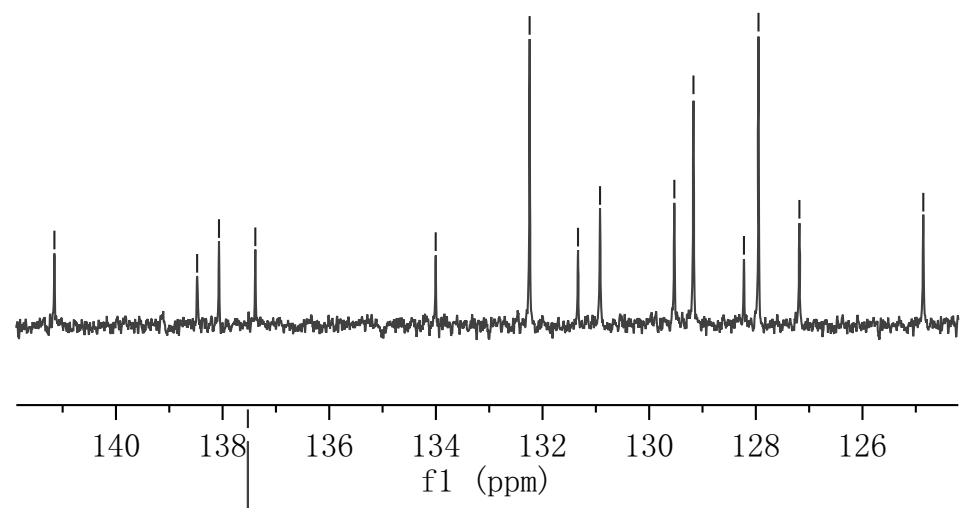

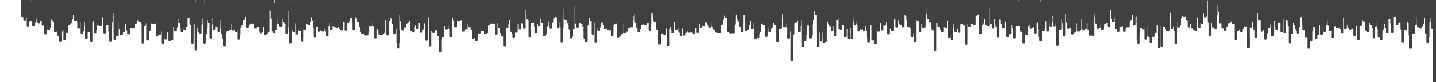

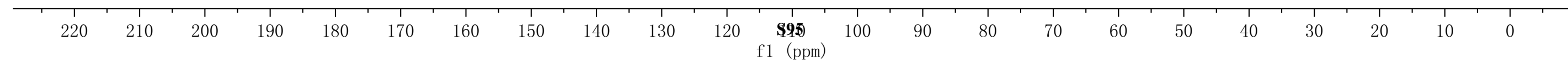




\begin{tabular}{|c|c|}
\hline Parameter & Value \\
\hline 1 Title & zgy $-4-147-\mathrm{H}$ \\
\hline 2 Origin & Bruker BioSpin Gmbl \\
\hline 3 Solvent & $\mathrm{CDC13}$ \\
\hline 4 Temperature & 298.0 \\
\hline 5 Number of Scans & 13 \\
\hline 6 Acquisition Tinfe & 4. 0894 \\
\hline 7 Acquisition Date & 2019-12-28T09:38:30 \\
\hline \multicolumn{2}{|c|}{8 Spectrometer Frequency 400.13} \\
\hline 9 Spectral Width & 8012.8 \\
\hline
\end{tabular}

20
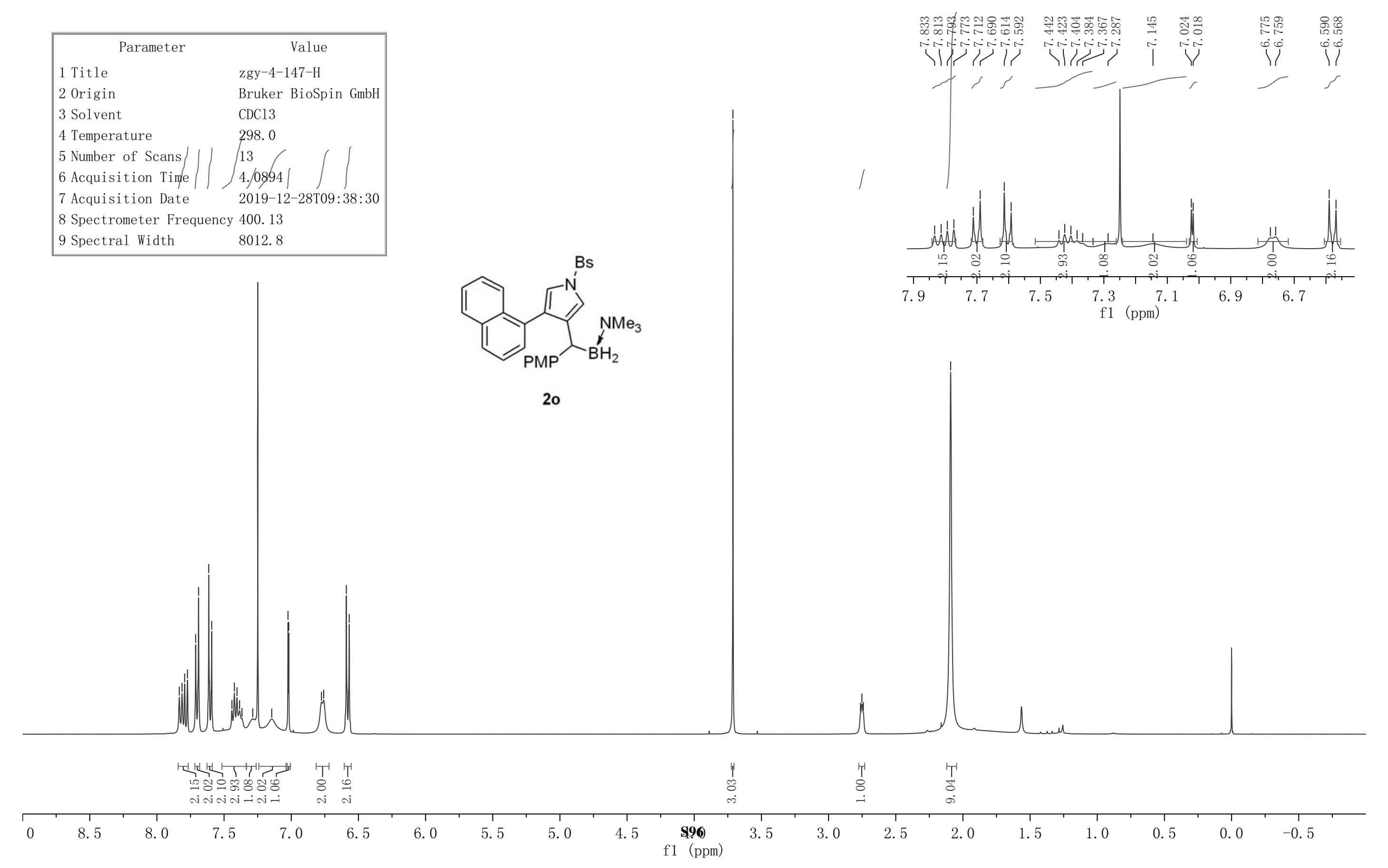


\begin{tabular}{|ll|}
\hline \multicolumn{1}{|c|}{ Parameter } & \multicolumn{1}{c|}{ Value } \\
1 Title & ZGY-4-147-C \\
2 Origin & Bruker BioSpin GmbH \\
3 Solvent & CDC13 \\
4 Temperature & 300.0 \\
5 Number of Scans & 74 \\
6 Acquisition Time & 1.3631 \\
7 Acquisition Date & $2019-12-27$ T10:32:37 \\
8 Spectrometer Frequency 100.61 \\
9 Spectral Width & 24038.5 \\
\hline
\end{tabular}
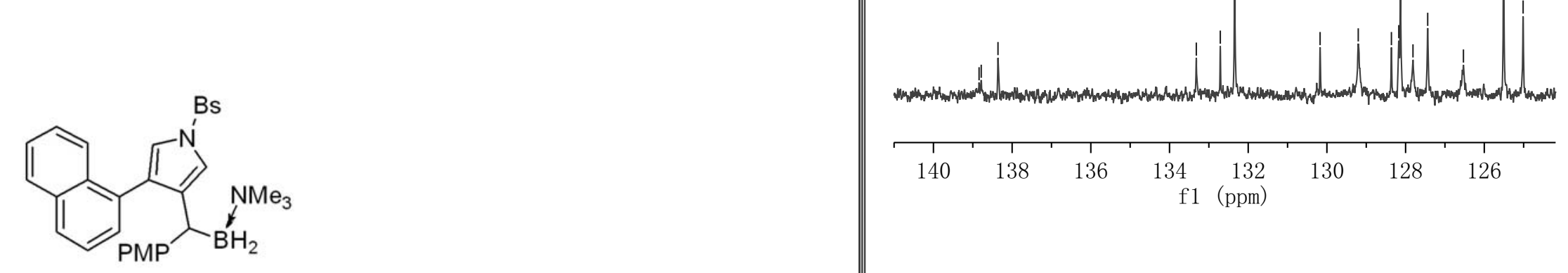

20

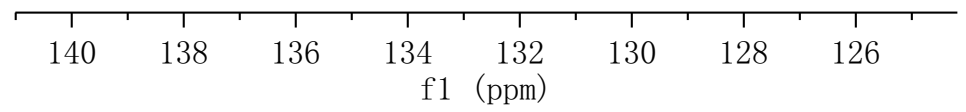

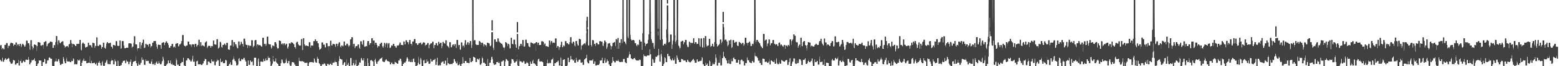

\begin{tabular}{|c|c|c|c|c|c|c|c|c|c|c|c|c|c|c|c|c|c|c|c|c|c|c|c|}
\hline 0 & \multicolumn{2}{|c|}{210} & 200 & 190 & 180 & 170 & 160 & $\frac{1}{150}$ & 140 & 130 & 120 & \multicolumn{2}{|c|}{$\begin{array}{ll}\mathbf{S 9 0} & 100\end{array}$} & 90 & $\begin{array}{l}1 \\
80\end{array}$ & 70 & 60 & $\begin{array}{l}1 \\
50\end{array}$ & 10 & 30 & \multicolumn{2}{|c|}{20} & 10 \\
\hline
\end{tabular}




\begin{tabular}{|c|c|}
\hline Parameter & Value \\
\hline 1 Title & zgy $-4-152$ \\
\hline 2 HrigiO & nruBer nikopi0 SmGb \\
\hline 3 oklve0t & $\mathrm{CDC} 13$ \\
\hline 4 Temperature & 298.0 \\
\hline 5 NumGer kf oca0s & 17 \\
\hline 6 Acquisitik0 Time & 4. 0894 \\
\hline 7 Acquisitik0 Date & $2019-12-26 \mathrm{~T} 10: 34: 54$ \\
\hline \multicolumn{2}{|c|}{8 opectrkmeter Freque0cy 400.13 } \\
\hline 9 opectral Width & 8012.8 \\
\hline
\end{tabular}
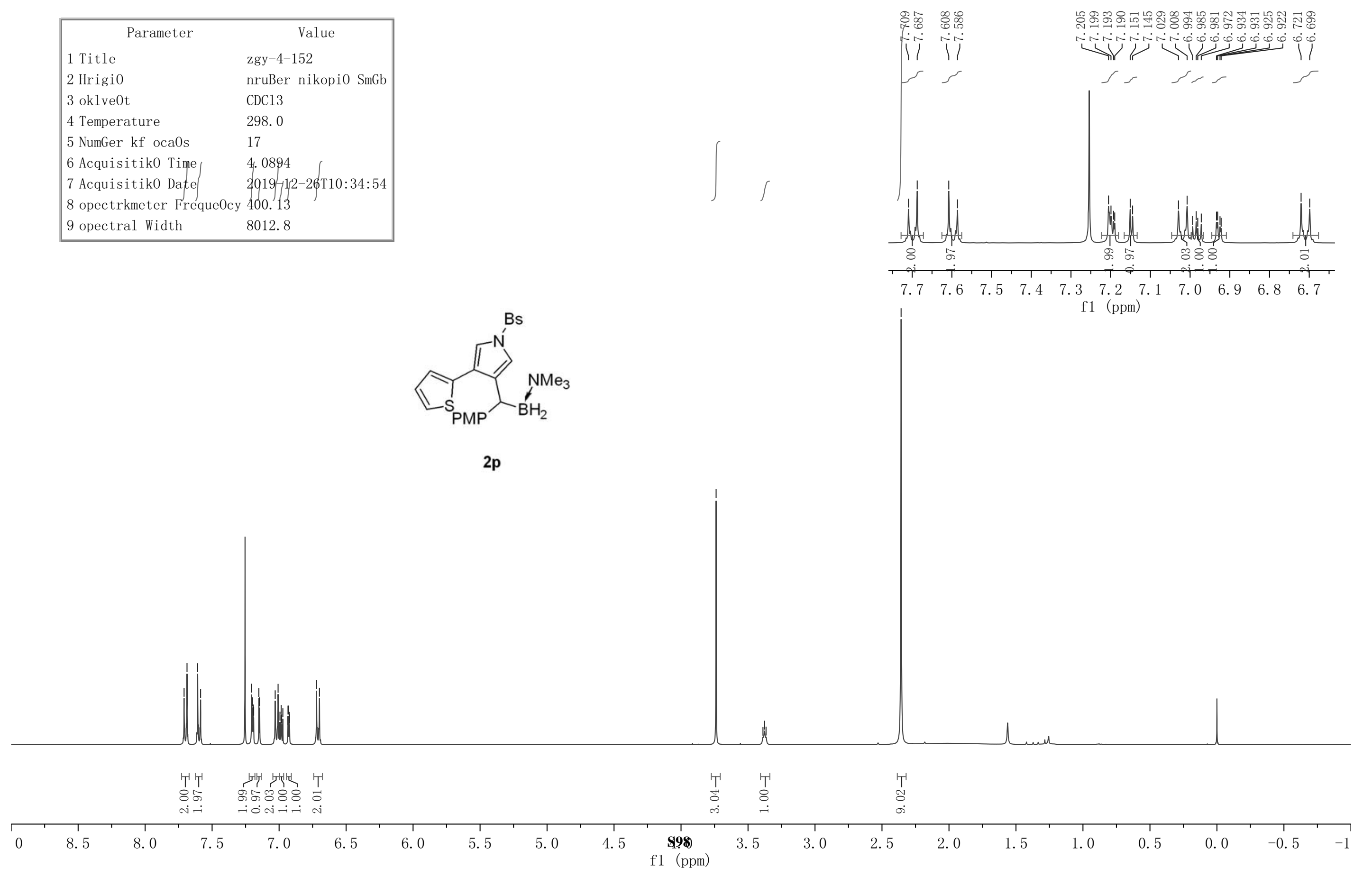


\begin{tabular}{|ll|}
\hline \multicolumn{1}{|c|}{ Parameter } & \multicolumn{1}{c|}{ Value } \\
1 Title & zgy-4-152-C \\
2 Origin & Bruker BioSpin GmbH \\
3 Solvent & CDC13 \\
4 Temperature & 300.0 \\
5 Number of Scans & 62 \\
6 Acquisition Time & 1.3631 \\
7 Acquisition Date & $2019-12-26 \mathrm{~T} 10: 38: 32$ \\
8 Spectrometer Frequency 100.61 \\
9 Spectral Width & 24038.5 \\
\hline
\end{tabular}
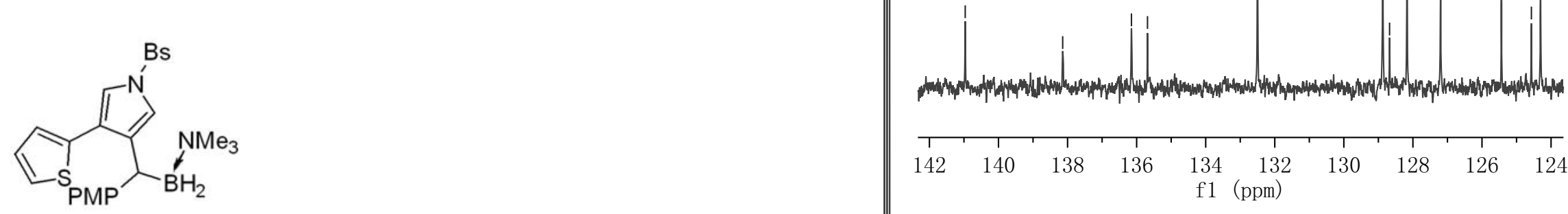

$2 p$

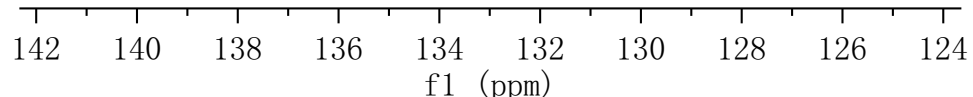

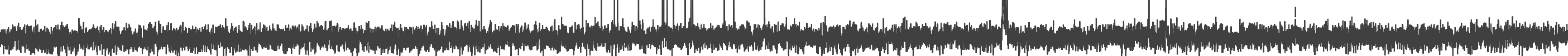

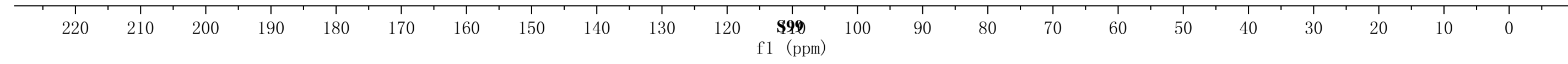




\begin{tabular}{|ll|}
\hline \multicolumn{1}{|c|}{ Parameter } & \multicolumn{1}{c|}{ Value } \\
1 Title & zgy-4-155-H \\
2 Origin & Bruker BioSpin GmbH \\
3 Solvent & CDC13 \\
4 Temperature & 298.0 \\
5 Number of Scans & 11 \\
6 Acquisition Time & 4.0894 \\
7 Acquisition Date & 2019-12-25T1@:23:47 \\
8 Spectrometer Frequency 400.13 \\
9 Spectral Width & 8012.8 \\
\hline
\end{tabular}

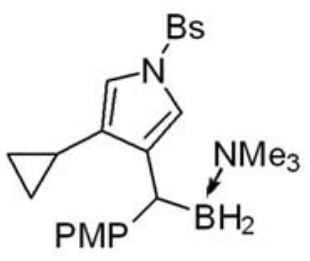

$2 q$

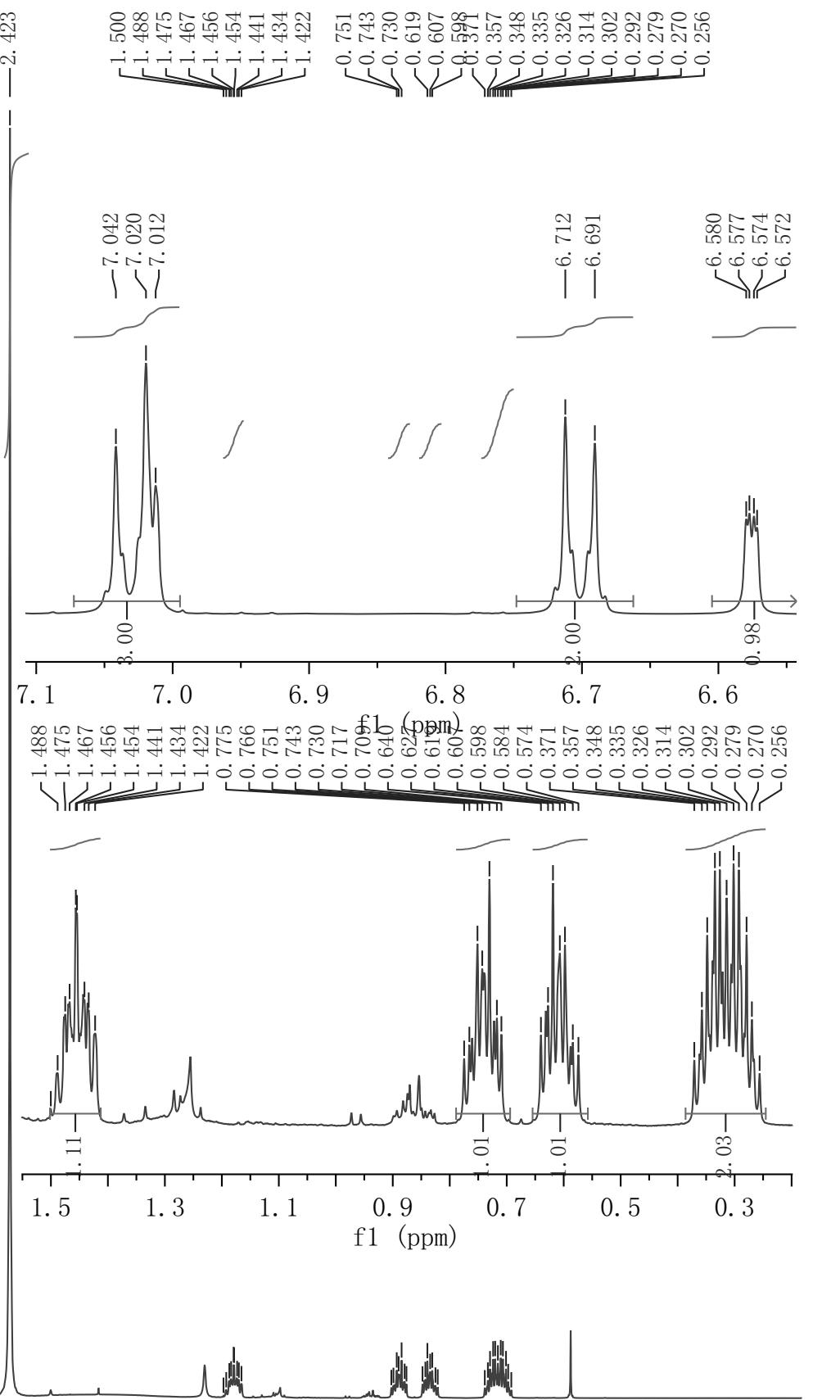

\begin{tabular}{|c|c|c|c|c|c|c|c|c|c|c|c|c|c|c|c|c|c|c|c|c|}
\hline & & & 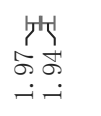 & $\begin{array}{l}T^{\prime} \\
8 \\
\dot{0}\end{array}$ & 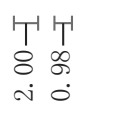 & & & & & & & $\begin{array}{l}\text { T' } \\
8 \\
\dot{-}\end{array}$ & $\begin{array}{l}1 \\
1 \\
8 \\
0\end{array}$ & & $\begin{array}{l}T^{\top} \\
= \\
-\end{array}$ & & 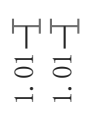 & $\begin{array}{l}{ }^{1} 1 \\
\infty \\
\infty \\
i\end{array}$ & & \\
\hline 0 & 8.5 & 8.0 & 7.5 & 7.0 & 6.5 & 6.0 & 5.5 & 5.0 & 4.5 & $\begin{array}{c} \\
\text { S1.00 } \\
\mathrm{f} 1 \quad(\mathrm{ppm})\end{array}$ & 3.5 & 3.0 & 2.5 & 2.0 & 1.5 & 1.0 & 0.5 & & 0.0 & -0.5 \\
\hline
\end{tabular}




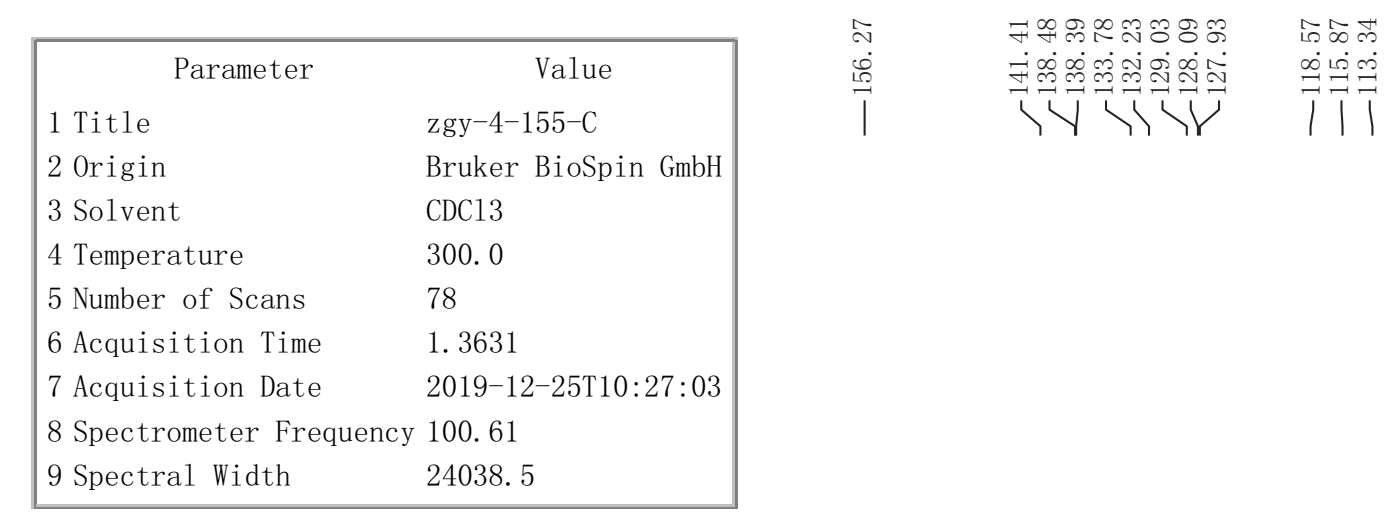
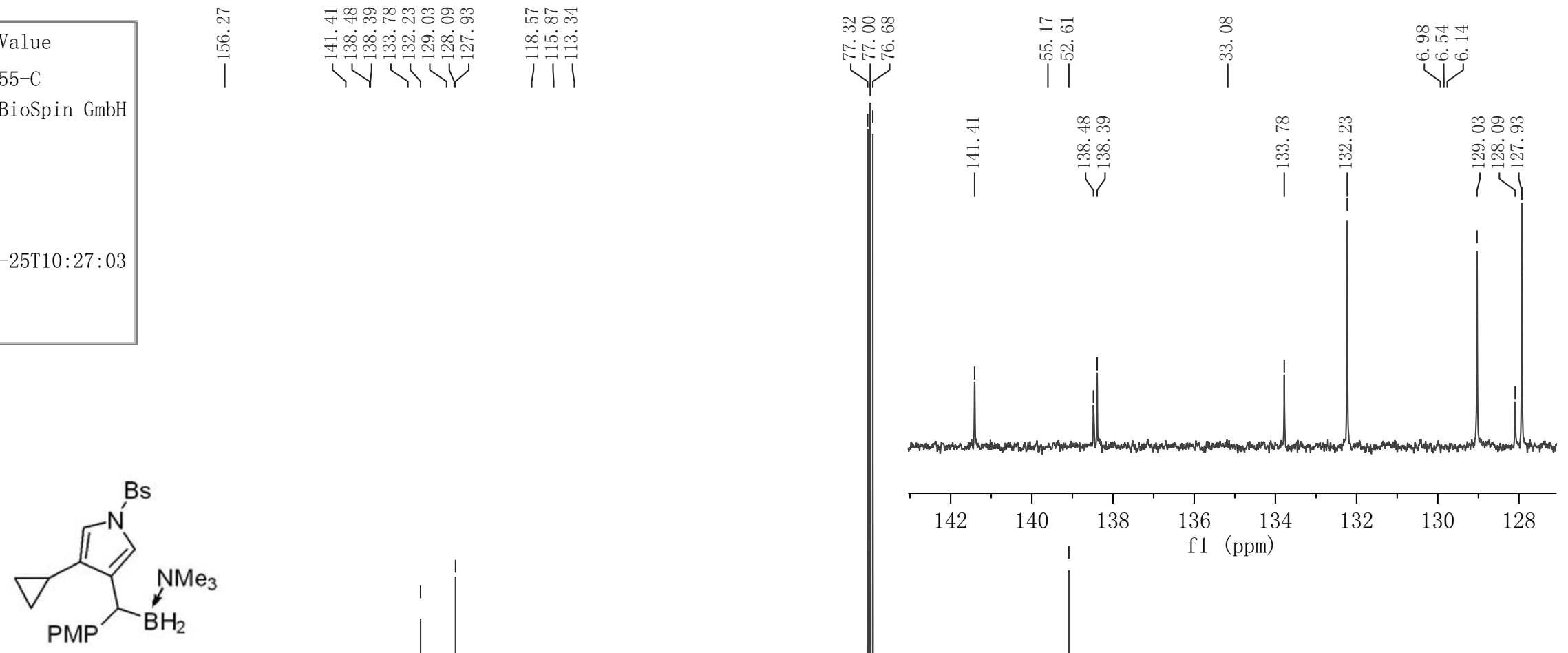

$2 q$
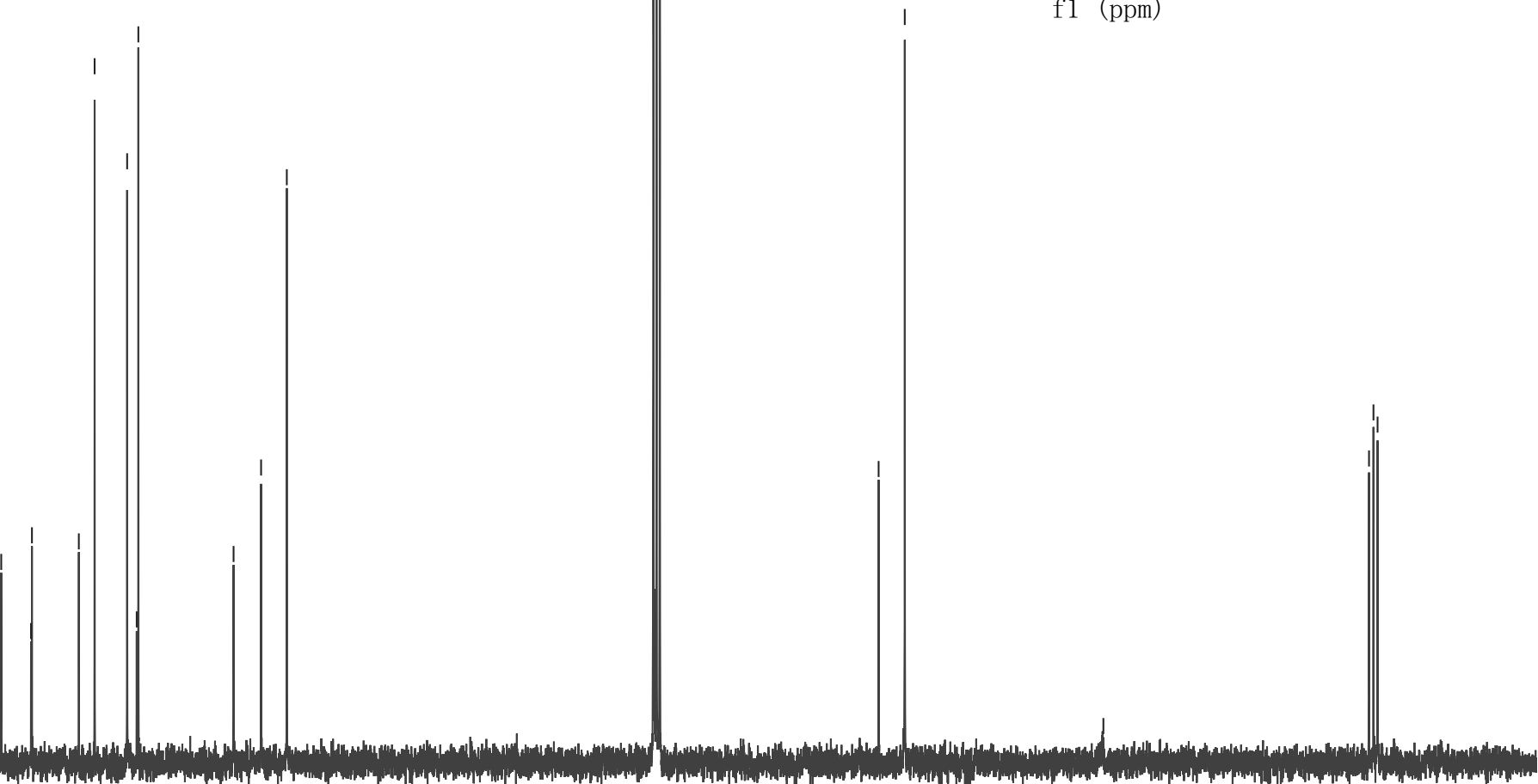

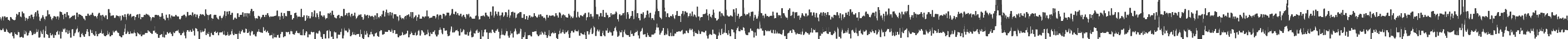

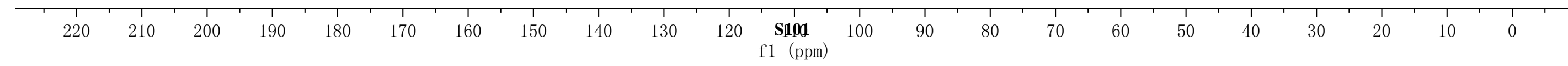




\begin{tabular}{|c|c|}
\hline Parameter & Value \\
\hline 1 Title & zgy $-5-198-2-\mathrm{H}$ \\
\hline 2 Origin & Bruker BioSpin GmbH \\
\hline 3 Solvent & CDC13 \\
\hline 4 Temperature & 298.0 \\
\hline 5 Number of Scans & \\
\hline 6 Acquisition Time & 4.0894 \\
\hline 7 Acquisition Date & 2020-09-17T14:02:24 \\
\hline 8 Spectrometer Frec & су 400.13 \\
\hline 9 Spectral Width & 8012.8 \\
\hline
\end{tabular}
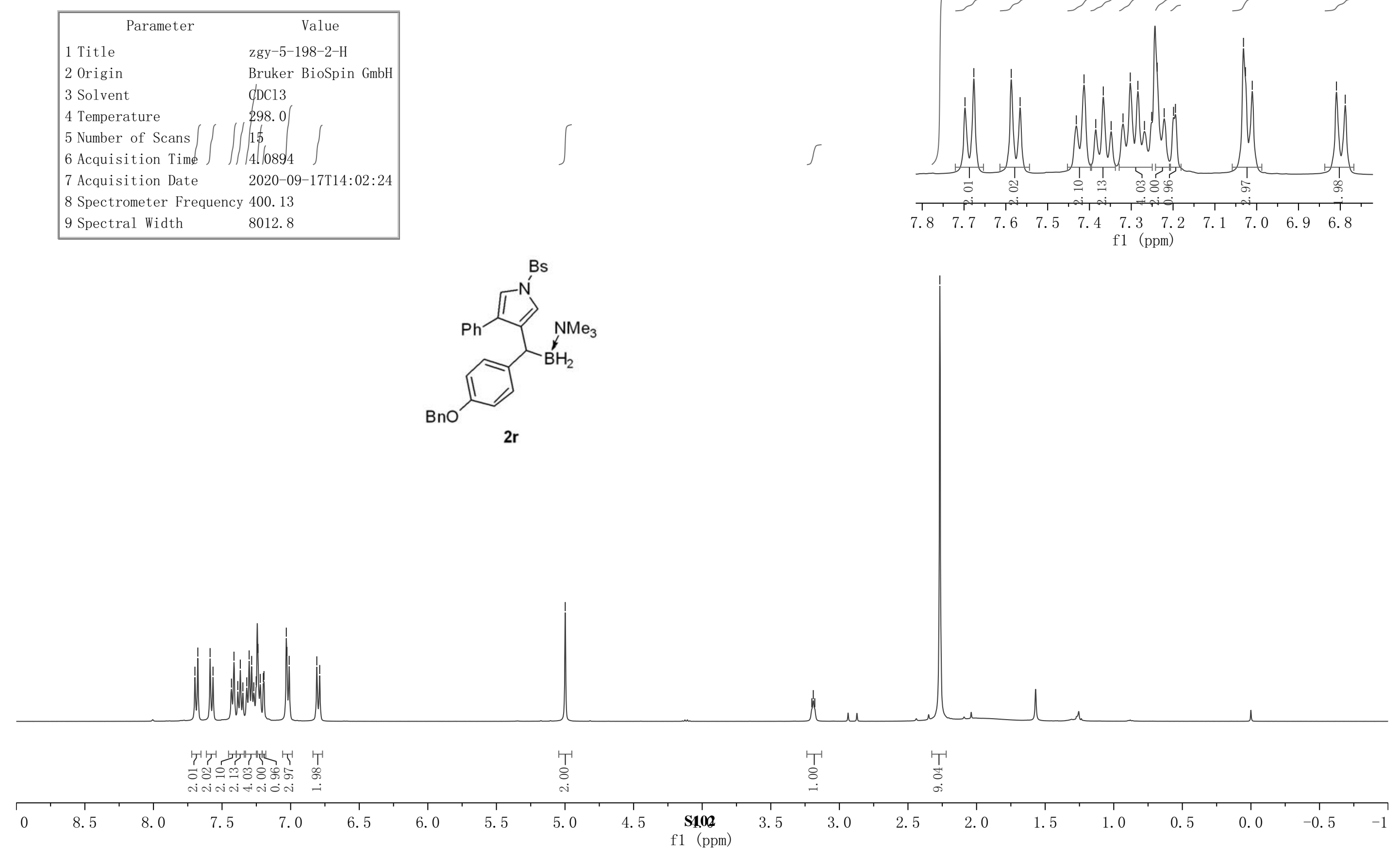


\begin{tabular}{|ll|}
\hline \multicolumn{1}{|c|}{ Parameter } & \multicolumn{1}{c|}{ Value } \\
1 Title & zgy-5-198-2-CC \\
2 Origin & Bruker BioSpin GmbH \\
3 Solvent & CDC13 \\
4 Temperature & 300.0 \\
5 Number of Scans & 133 \\
6 Acquisition Time & 1.3631 \\
7 Acquisition Date & $2020-09-17 \mathrm{~T} 14: 05: 59$ \\
8 Spectrometer Frequency & 100.61 \\
9 Spectral Width & 24038.5 \\
\hline
\end{tabular}

gy-5-198-2-CC

| ।
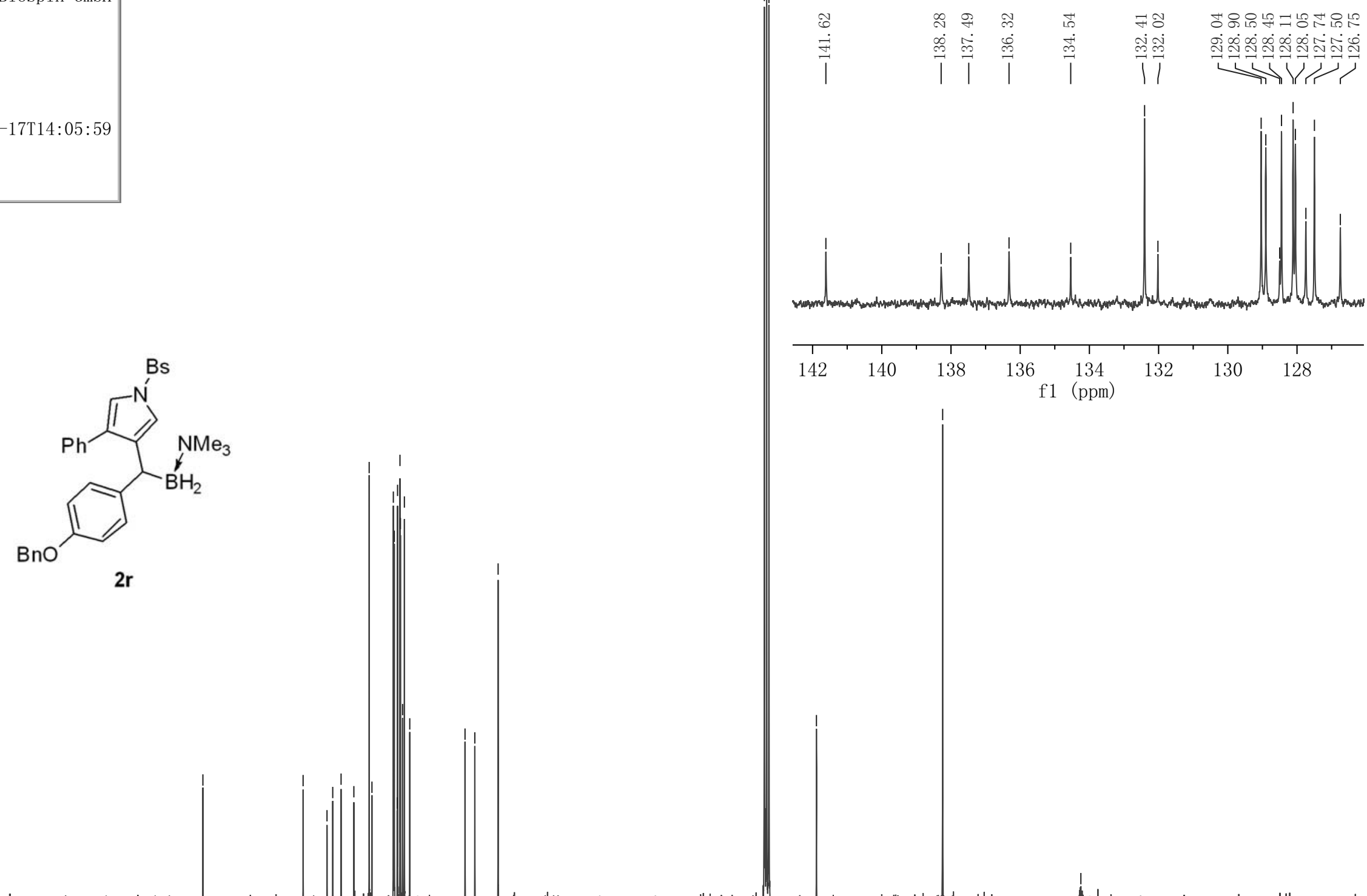

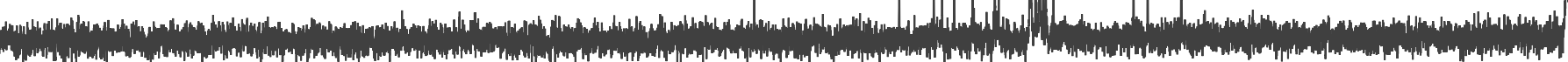

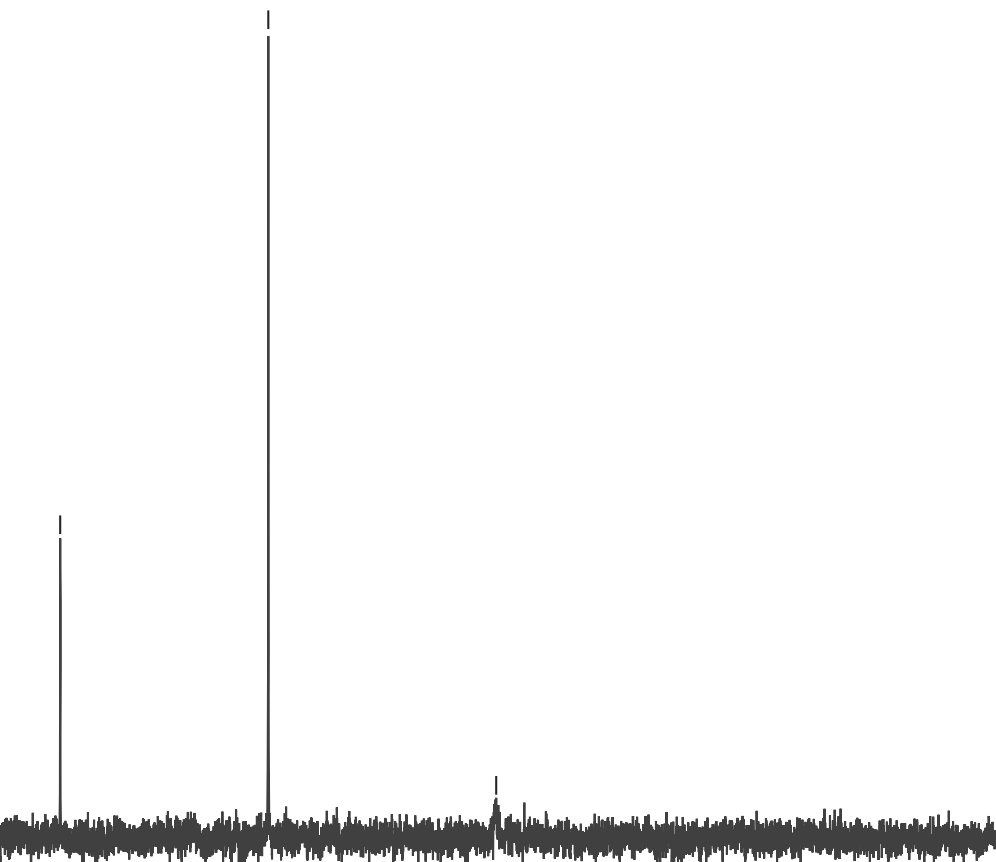

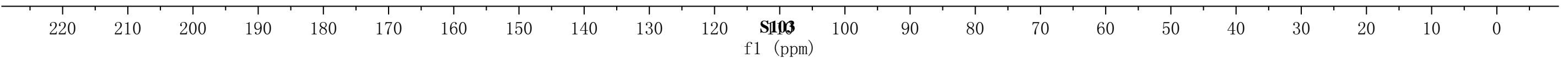




\begin{tabular}{|c|c|}
\hline Parameter & Value \\
\hline 1 Title & zgy $-4-170-H$ \\
\hline 2 Origin & Bruker BioSpin GmbH \\
\hline 3 Solvent & $\mathrm{CDC} 13$ \\
\hline 4 Temperature & 298.0 \\
\hline 5 Number of Scans & 13 \\
\hline 6 Acquisition Time & 4. 0894 \\
\hline 7 Acquisition Date & 2020-01-01T10:40:54 \\
\hline \multicolumn{2}{|c|}{8 Spectrometer Frequency 400.13} \\
\hline 9 Spectral Width & 8012.8 \\
\hline
\end{tabular}
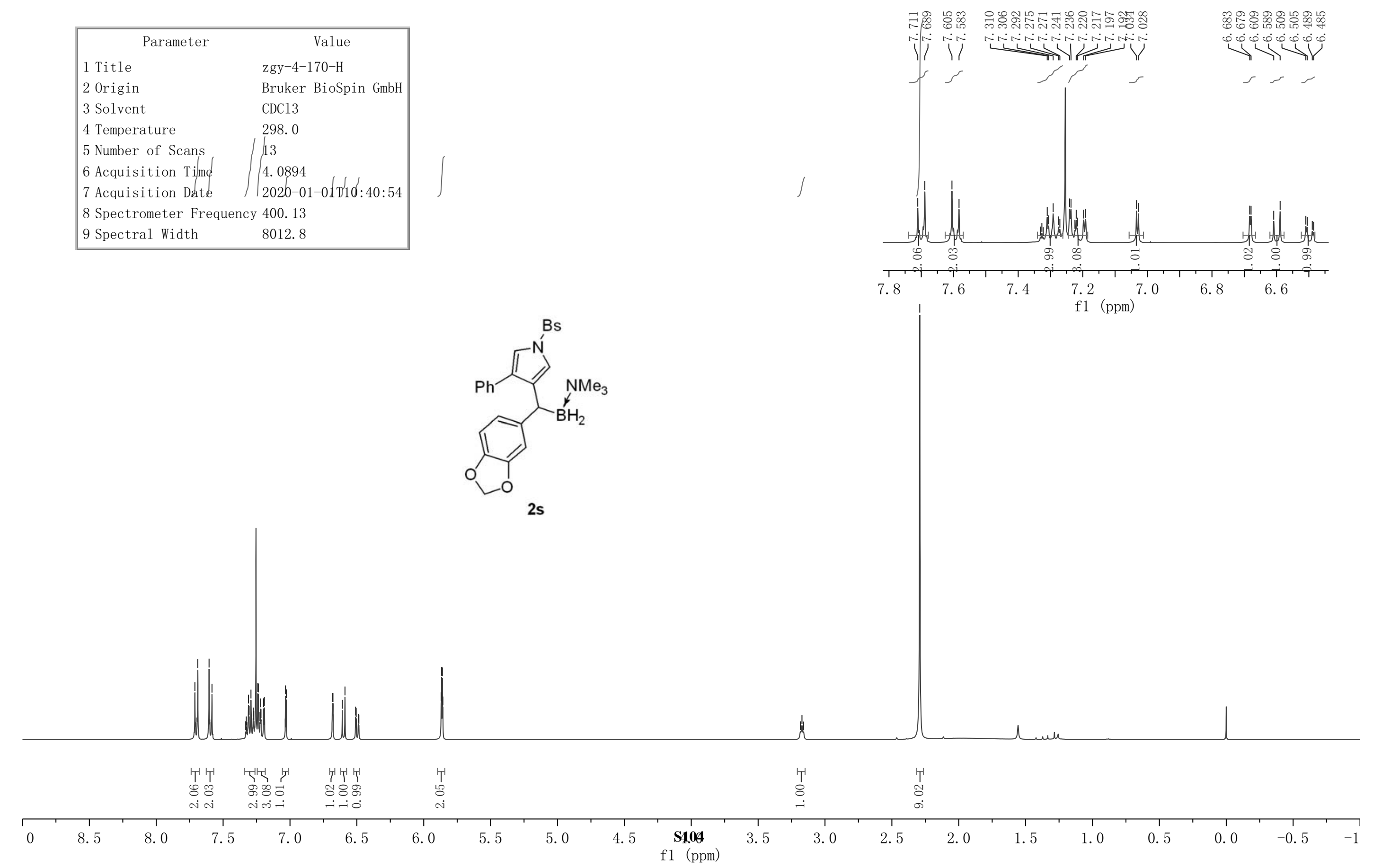


\begin{tabular}{|ll|}
\hline \multicolumn{1}{|c|}{ Parameter } & \multicolumn{1}{c|}{ Value } \\
1 Title & zgy-4-170-C \\
2 Origin & Bruker BioSpin GmbH \\
3 Solvent & CDC13 \\
4 Temperature & 300.0 \\
5 Number of Scans & 56 \\
6 Acquisition Time & 1.3631 \\
7 Acquisition Date & $2020-01-01 \mathrm{~T} 10: 44: 31$ \\
8 Spectrometer Frequency & 100.61 \\
9 Spectral Width & 24038.5 \\
\hline
\end{tabular}

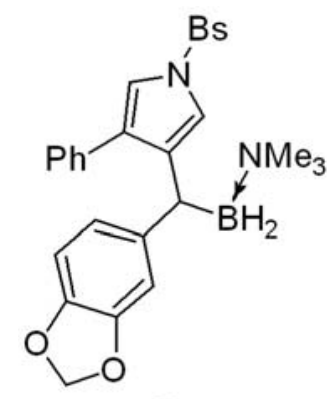

2s

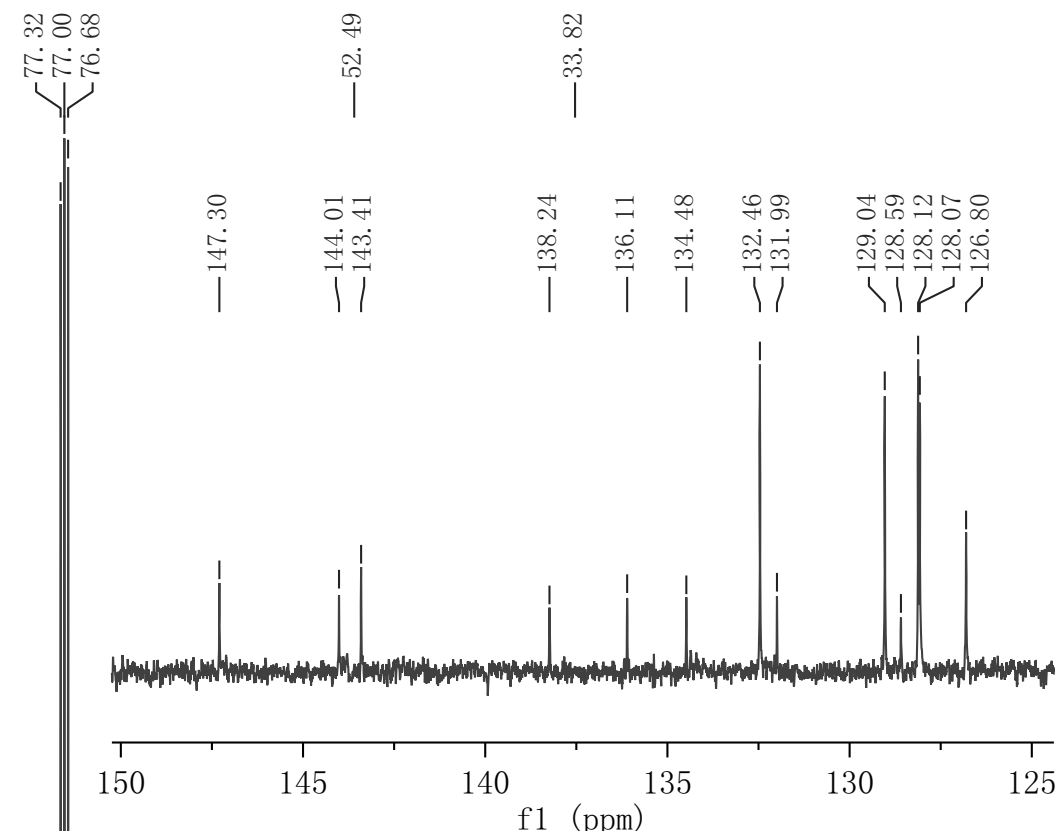

f1 (ppm)

125

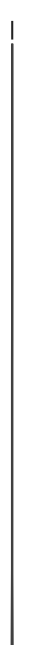

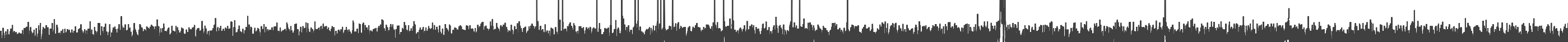

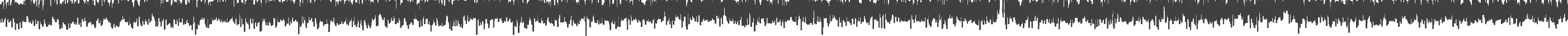

100

90

80

70

$70 \quad 60$

$60 \quad 50$

$50 \quad 4$

$\begin{array}{ll}1 & 1\end{array}$

1
$30 \quad 20$




\begin{tabular}{|c|c|}
\hline Parameter & Value \\
\hline 1 Title & zgy $-5-198-1-H$ \\
\hline 2 Origin & Bruker BioSpin GmbH \\
\hline 3 Solvent & $\mathrm{CDC} 13$ \\
\hline 4 Temperature & 299.3 \\
\hline 5 Number of Scans & 33 \\
\hline 6 Acquisition Time & 3.9846 \\
\hline 7 Acquisition Date & 2020-09-13T $10: 15: 43$ \\
\hline \multicolumn{2}{|c|}{8 Spectrometer Frequency 400. 03} \\
\hline 9 Spectral Width & 8223.7 \\
\hline
\end{tabular}

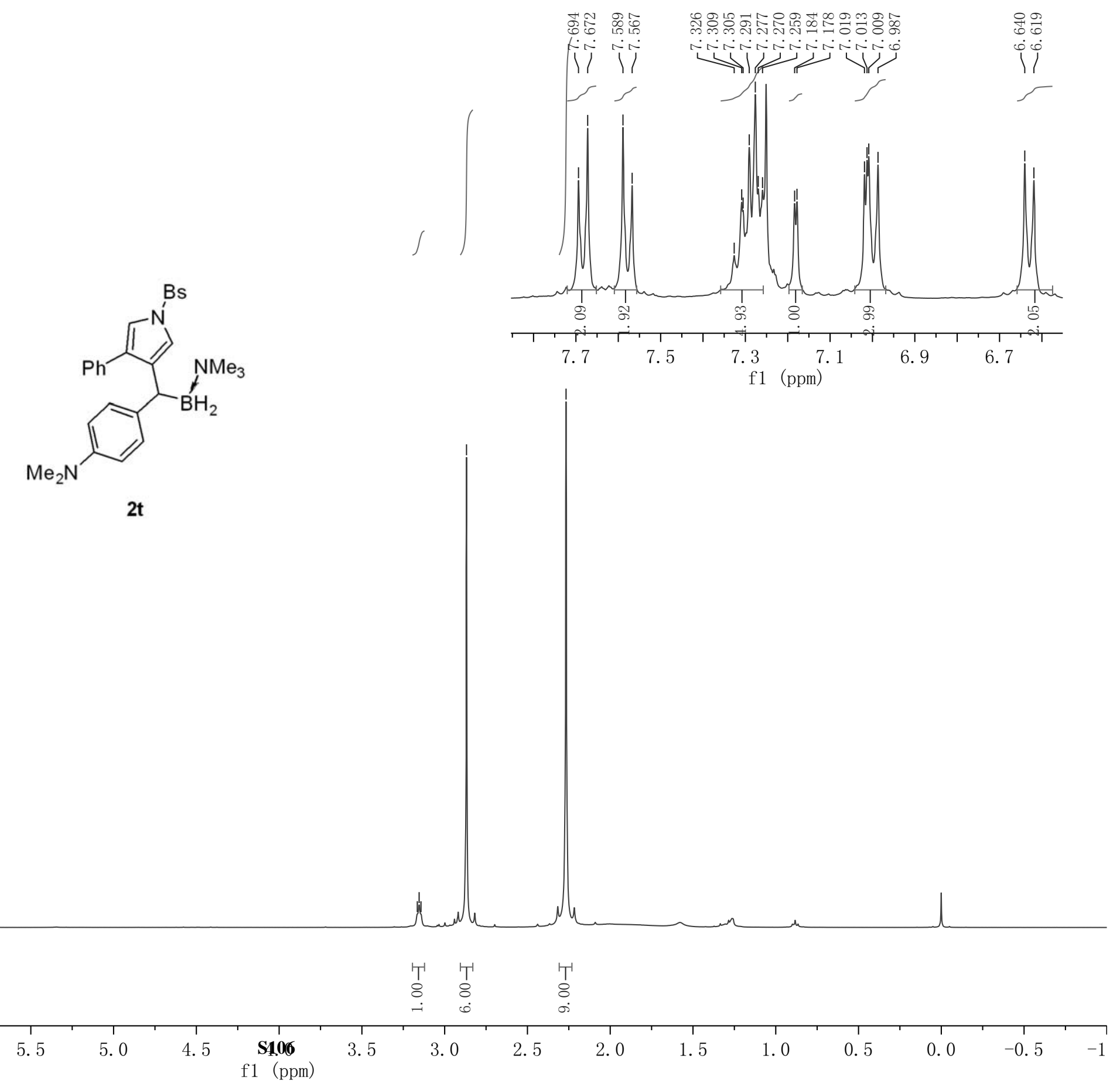




\begin{tabular}{|ll|}
\hline \multicolumn{1}{|c|}{ Parameter } & \multicolumn{1}{c|}{ Value } \\
1 Title & zgy-5-198-1-C \\
2 Origin & Bruker BioSpin GmbH \\
3 Solvent & CDC13 \\
4 Temperature & 299.3 \\
5 Number of Scans & 185 \\
6 Acquisition Time & 1.3631 \\
7 Acquisition Date & $2020-09-13 T 10: 20: 36$ \\
8 Spectrometer Frequency 100.59 \\
9 Spectral Width & 24038.5 \\
\hline
\end{tabular}
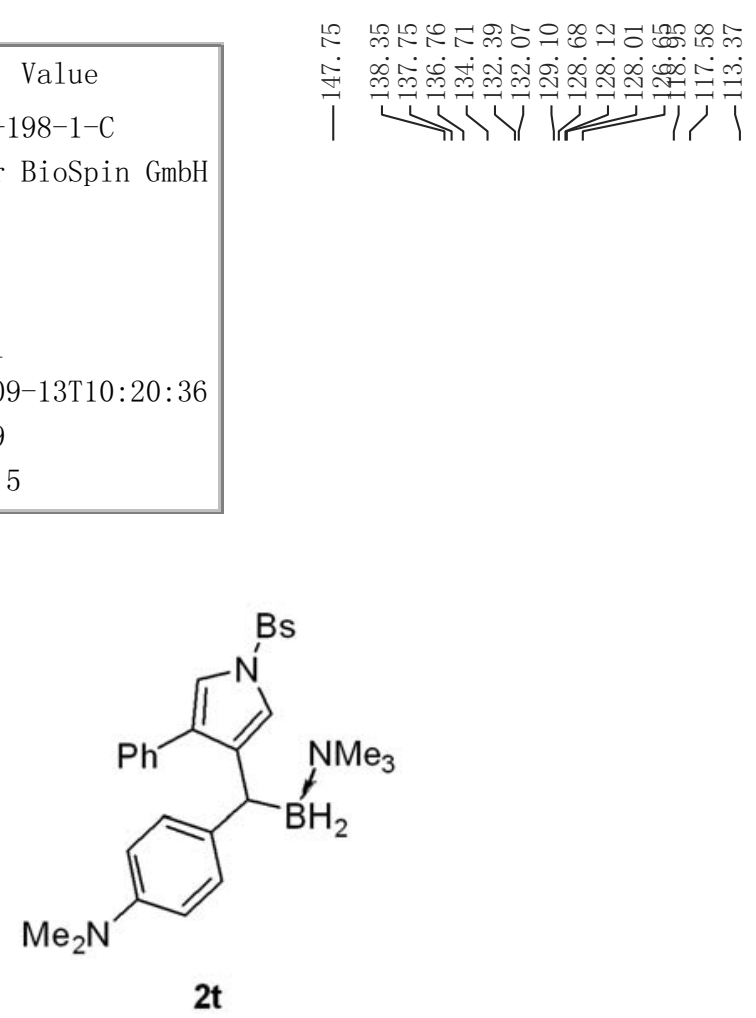

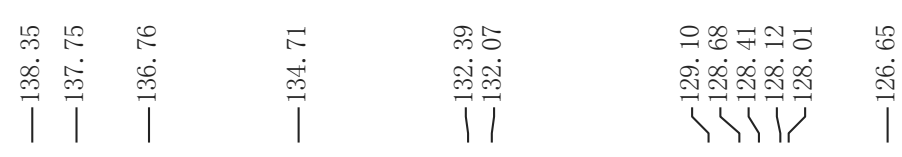
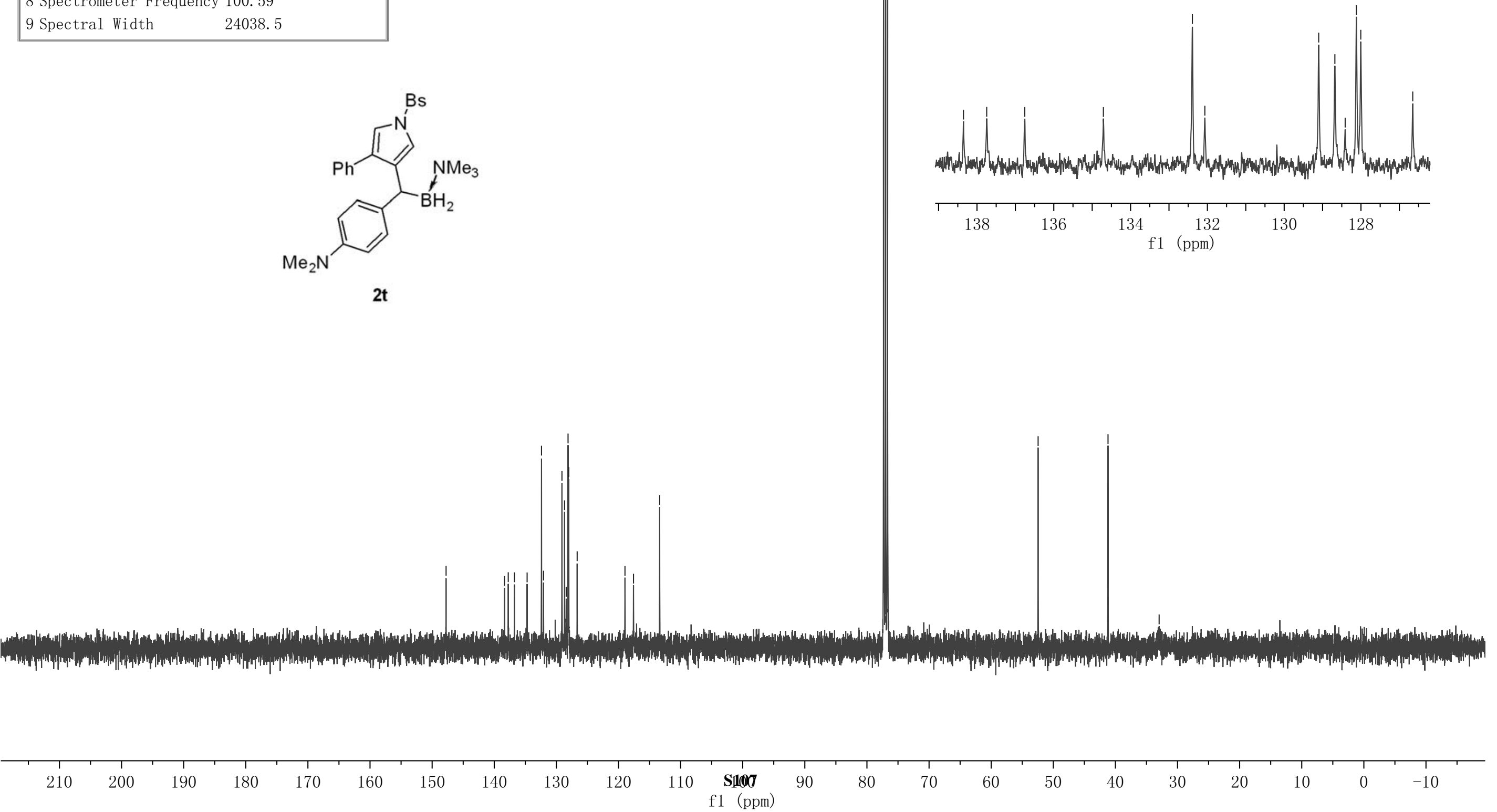


\begin{tabular}{|ll|}
\hline \multicolumn{1}{|c|}{ Parameter } & \multicolumn{1}{c|}{ Value } \\
1 Title & zgy-4-174-H \\
2 Origin & Bruker BioSpin GmbH \\
3 Solvent & CDC13 \\
4 Temperature & 298.0 \\
5 Number of Scans & 11 \\
6 Acquisition Time $\iint$ & 4.0894 \\
7 Acquisition Date & $2020-01 / 01 T 15: 28: 11$ \\
8 Spectrometer Frequency & 400.13 \\
9 Spectral Width & 8012.8 \\
\hline
\end{tabular}

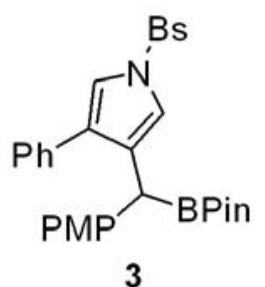




\begin{tabular}{|ll|}
\hline \multicolumn{1}{|c|}{ Parameter } & \multicolumn{1}{c|}{ Value } \\
1 Title & zgy-4-174-C \\
2 Origin & Bruker BioSpin GmbH \\
3 Solvent & CDC13 \\
4 Temperature & 300.0 \\
5 Number of Scans & 36 \\
6 Acquisition Time & 1.3631 \\
7 Acquisition Date & $2020-01-01 \mathrm{~T} 15: 31: 14$ \\
8 Spectrometer Frequency 100.61 \\
9 Spectral Width & 24038.5 \\
\hline
\end{tabular}

9 Spectral width

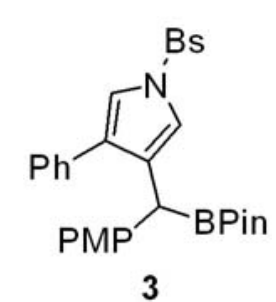

3

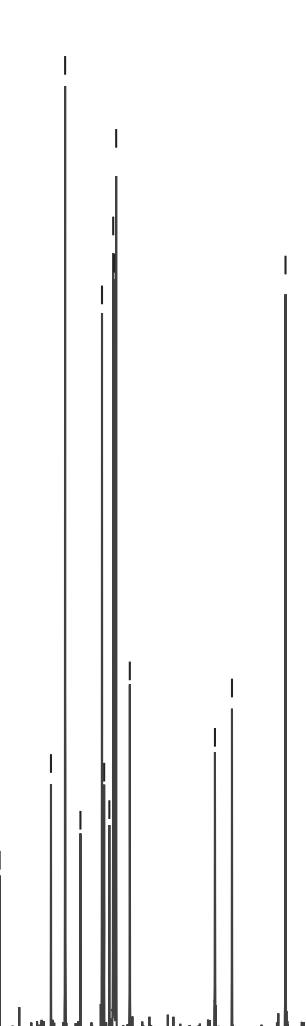

Him
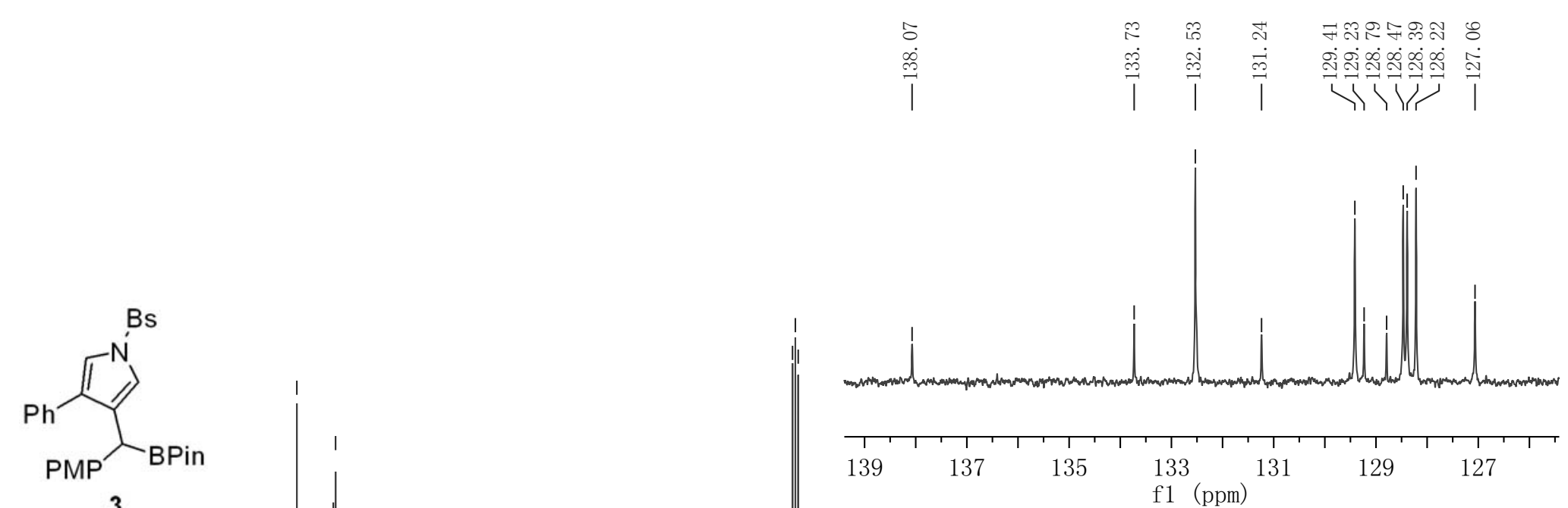

\begin{tabular}{|c|c|c|c|c|c|c|c|c|c|c|c|c|c|c|c|c|c|c|c|c|c|c|}
\hline $\begin{array}{c}1 \\
220\end{array}$ & 210 & $\begin{array}{c}1 \\
200\end{array}$ & $\begin{array}{c}1 \\
190\end{array}$ & $\begin{array}{c}1 \\
180\end{array}$ & 170 & 160 & 150 & $\begin{array}{r}1 \\
140\end{array}$ & $\begin{array}{r}1 \\
130\end{array}$ & $\begin{array}{c}120 \\
120\end{array}$ & $\begin{array}{c}\text { S1109 } \\
\text { f1 (ppm) }\end{array}$ & 100 & 90 & 80 & 70 & 60 & $\begin{array}{l}1 \\
50\end{array}$ & 10 & $\begin{array}{l}1 \\
30\end{array}$ & $\begin{array}{c}1 \\
20\end{array}$ & 10 & $\begin{array}{l}T \\
0\end{array}$ \\
\hline
\end{tabular}




\begin{tabular}{|c|c|}
\hline \multicolumn{2}{|l|}{ Parameter } \\
\hline 1 Title & $178-\mathrm{H}$ \\
\hline 2 Origin & Bruker BioSpin GmbH \\
\hline 3 Solvent & $\mathrm{QDCl} B$ \\
\hline 4 Temperature & 298.0 \\
\hline 5 Number of Scans & \\
\hline 6 Acquisition Time & 4. 0894 \\
\hline 7 Acquisition Date & $2020-01-07 \mathrm{~T} 10: 35: 11$ \\
\hline 8 Spectrometer Fre & 400.13 \\
\hline 9 Spectral Width & 8012.8 \\
\hline
\end{tabular}

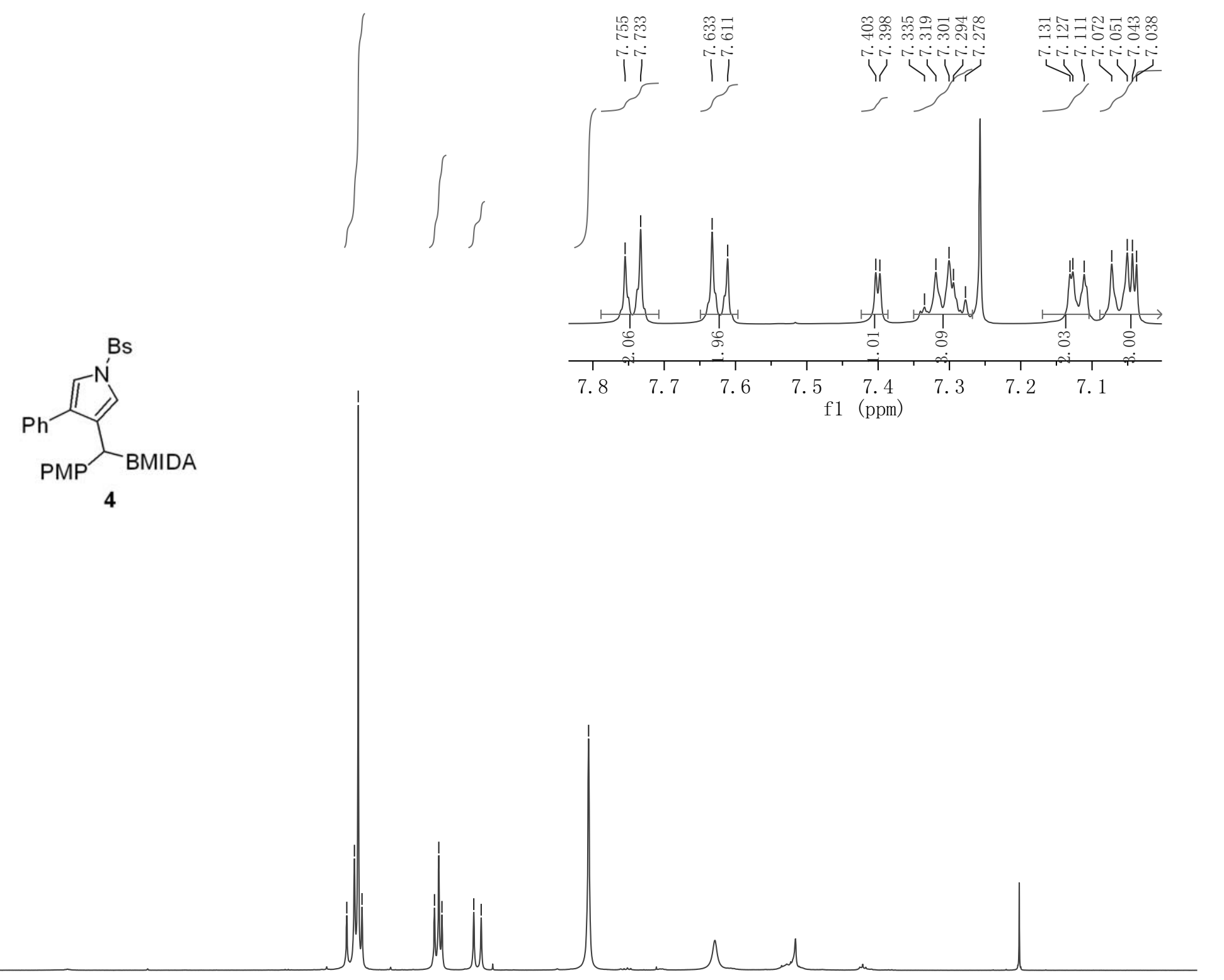

\begin{tabular}{|c|c|c|c|c|c|c|c|c|c|c|c|c|c|c|c|c|c|c|c|}
\hline & & $\begin{array}{l}T^{1} \\
\stackrel{8}{8} \\
- \\
-\end{array}$ & 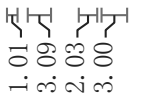 & $\begin{array}{l}\text { T' } \\
\overrightarrow{0} \\
\stackrel{i}{i}\end{array}$ & & & & & & & $\begin{array}{ll}T & T \\
8 & 9 \\
8 & 0 \\
i & 1\end{array}$ & 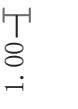 & $\begin{array}{l}T^{T} \\
\overrightarrow{0} \\
\dot{\infty}\end{array}$ & & & & & & \\
\hline 8.5 & 8.0 & 7.5 & 7. 0 & 6.5 & 6.0 & 5.5 & 5. 0 & 4.5 & $\begin{array}{c}\text { S410 } \\
\text { f1 (ppm) }\end{array}$ & 3.5 & & 3.0 & 2.5 & 2. 0 & 1.5 & 1.0 & 0.5 & 0.0 & -0.5 \\
\hline
\end{tabular}




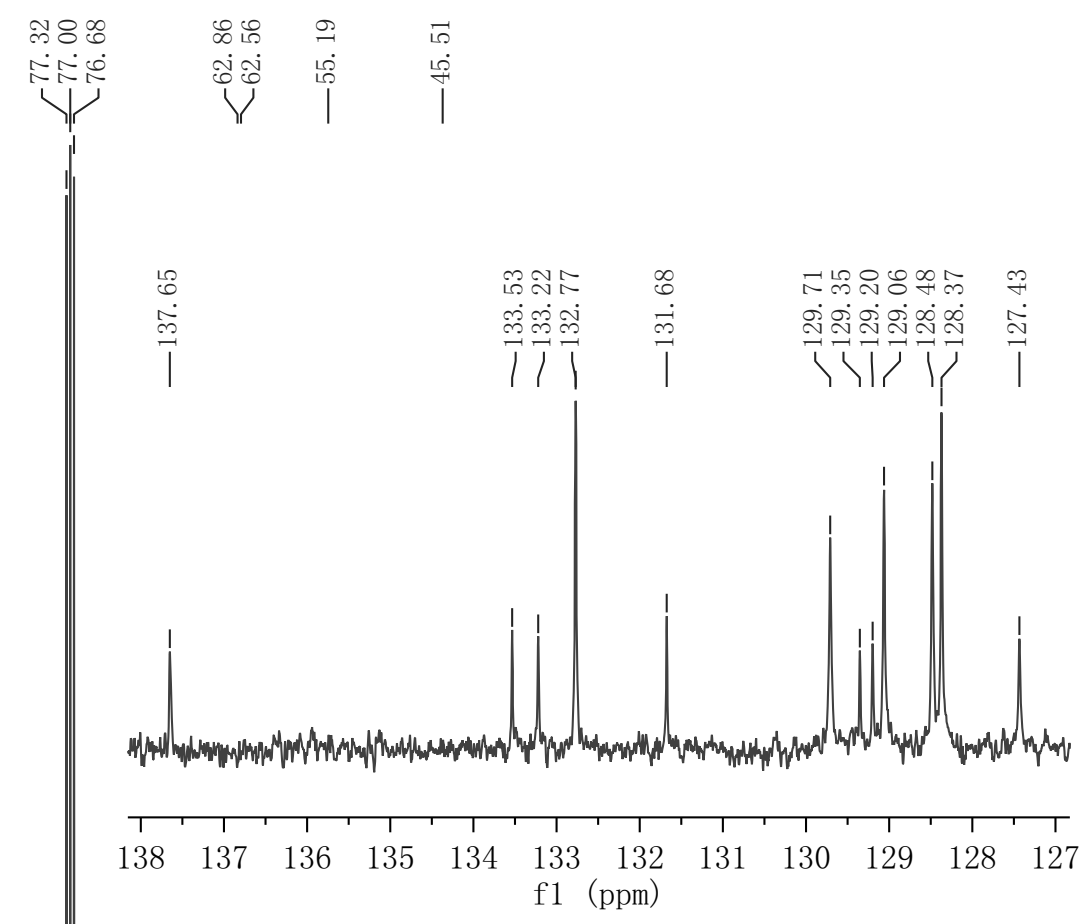

\begin{tabular}{|ll|}
\hline \multicolumn{1}{|c|}{ Parameter } & \multicolumn{1}{c|}{ Value } \\
1 Title & zgy-4-178-C \\
2 Origin & Bruker BioSpin GmbH \\
3 Solvent & CDC13 \\
4 Temperature & 300.0 \\
5 Number of Scans & 91 \\
6 Acquisition Time & 1.3631 \\
7 Acquisition Date & 2020-01-07T10:40:06 \\
8 Spectrometer Frequency 100.61 \\
9 Spectral Width & 24038.5 \\
\hline
\end{tabular}
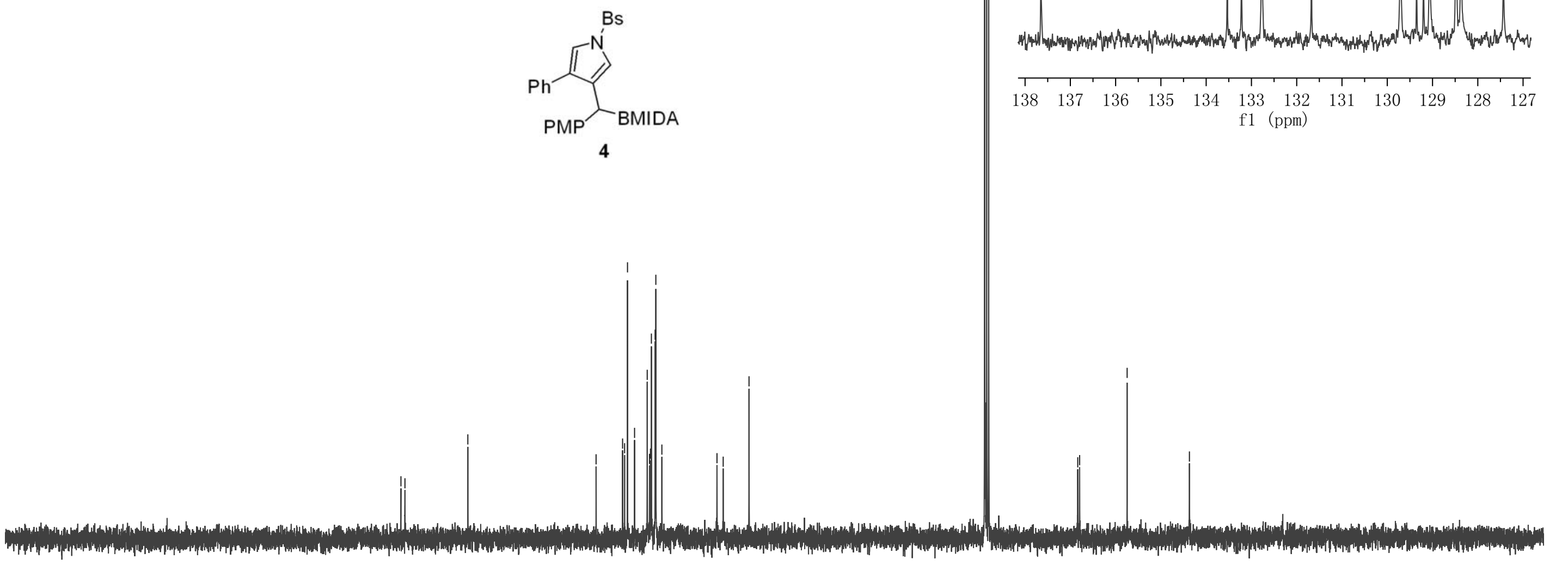

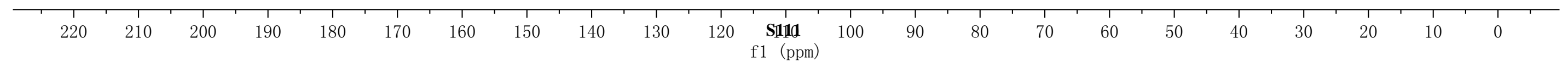




\begin{tabular}{|c|c|}
\hline Parameter & Value \\
\hline 1 Title & $\mathrm{zgy}-4-184-\mathrm{H}$ \\
\hline 2 Origin & Bruker BioSpin GmbH \\
\hline 3 Solvent & $\mathrm{CDCl3}$ \\
\hline 4 Temperature & 298.0 \\
\hline 5 Number of Scans & 20 \\
\hline 6 Acquisition Time & 4. 0894 \\
\hline 7 Acquisition Date & $2020-01-11 \mathrm{~T} 10: 06: 11$ \\
\hline \multicolumn{2}{|c|}{8 Spectrometer Frequency 400. 13} \\
\hline 9 Spectral Width & 8012.8 \\
\hline
\end{tabular}
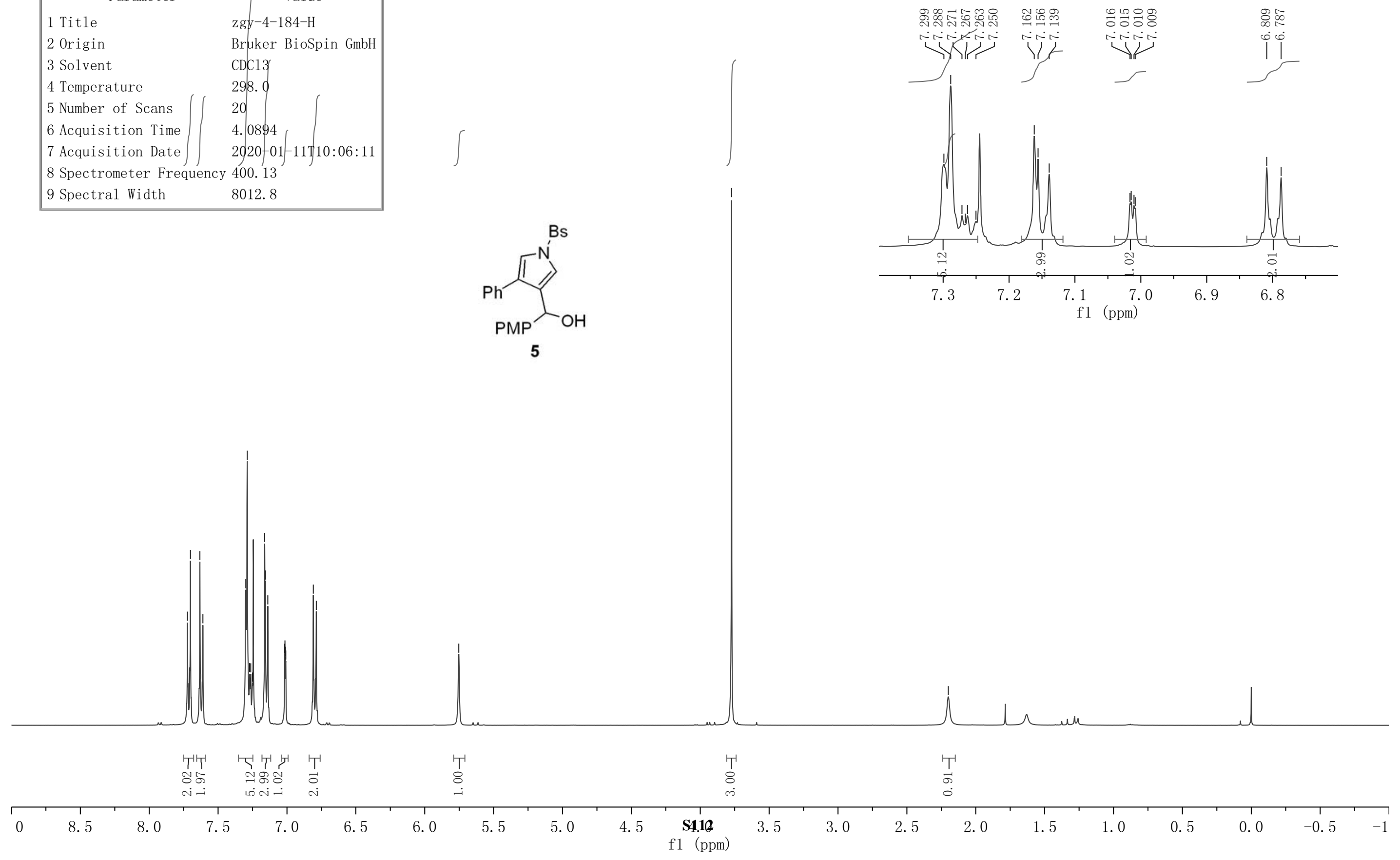


\begin{tabular}{|ll|}
\hline \multicolumn{1}{|c|}{ Parameter } & \multicolumn{1}{c|}{ Value } \\
1 Title & zgy-4-184-C \\
2 Origin & Bruker BioSpin GmbH \\
3 Solvent & CDC13 \\
4 Temperature & 300.0 \\
5 Number of Scans & 48 \\
6 Acquisition Time & 1.3631 \\
7 Acquisition Date & $2020-01-11 \mathrm{~T} 10: 10: 21$ \\
8 Spectrometer Frequency 100.61 \\
9 Spectral Width & 24038.5 \\
\hline
\end{tabular}
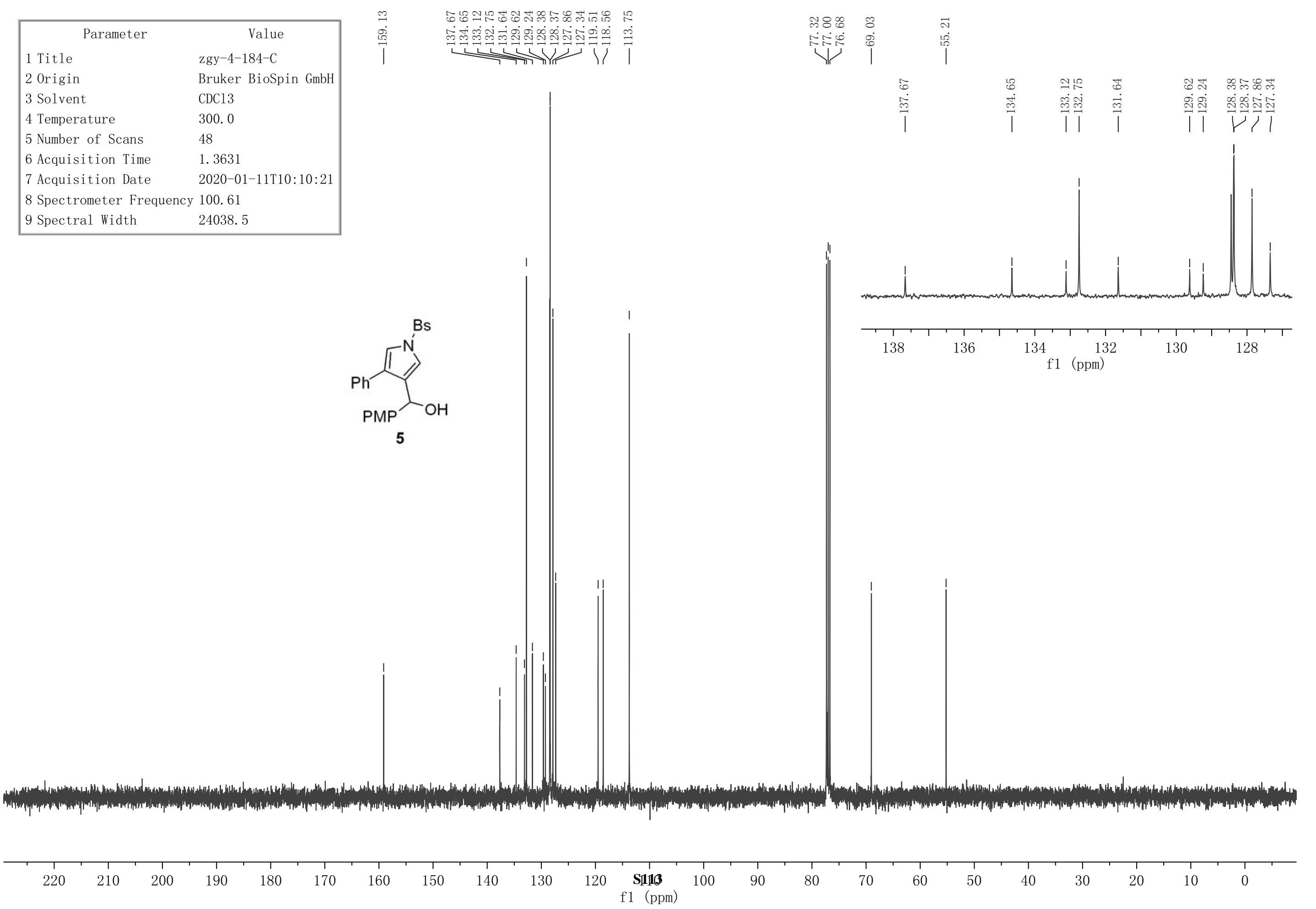